\title{
Construction of 1-Tetralols Bearing two Contiguous Quaternary Chiral Centers through a Rhodium-Catalyzed Enantioselective Desymmetrization Cascade Reaction
}

\author{
Aymane Selmani, Sylvain Darses* \\ PSL Université Paris, Chimie ParisTech - CNRS, Institute of Chemistry for Life and Health \\ Sciences (i-CLeHS), 11 rue Pierre et Marie Curie, 75005, Paris (France)
}

\section{Supporting Information}

\section{Table of contents}

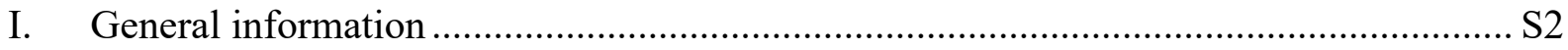

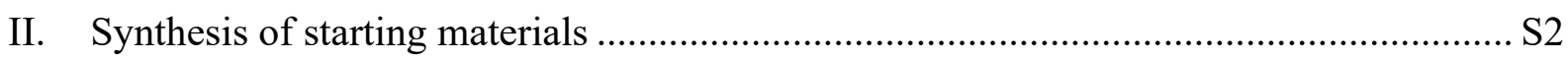

A. General procedure (1) for the preparation of starting materials ................ S2

B. General procedure (2) for the preparation of starting materials ................ S4

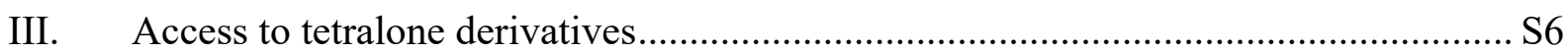
A. General procedure (3) for the preparation of racemic 1-tetralol derivatives S6
B. General procedure (4) for the preparation of chiral 1-tetralol derivatives.. S6

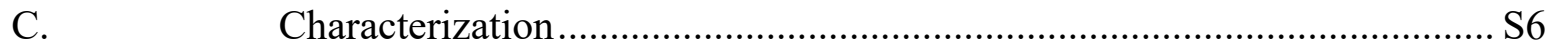

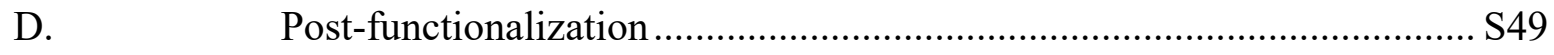

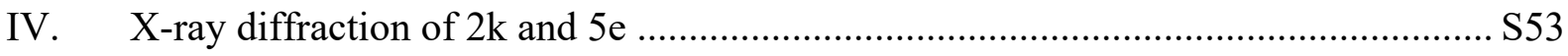

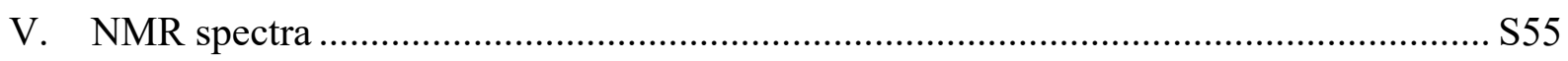




\section{General information}

All reactions were performed under an argon atmosphere. Reaction vessels were oven-dried, cooled under vacuum and flushed with argon before use. $\mathrm{MeOH}$ was distilled over sodium, water was distilled, DCM and THF was dried over alumina columns in an Innovative Technologies apparatus. All the solvents for catalysis were degassed prior to use. Reagents were obtained from commercial suppliers and used without further purification.

Reactions were monitored by thin layer chromatography (TLC) carried out on silica gel plates (Merck Kieselgel 60 F254); spots were detected with UV light and/or by staining with $\mathrm{KMnO}_{4}$ solution. Flash column chromatography was performed on silica gel (Merck, spherical, neutral, 40-60 $\mu \mathrm{m})$.

${ }^{1} \mathrm{H}$ NMR, ${ }^{19} \mathrm{~F}$ NMR and ${ }^{13} \mathrm{C}\left\{{ }^{1} \mathrm{H}\right\}$ NMR were recorded on a Bruker Avance 400 instrument in $\mathrm{CDCl}_{3}$. Chemical shifts are reported in delta $(\delta)$ units part per million (ppm). Residual $\mathrm{CHCl}_{3}$ $\left(\delta\right.$ 7.26) and $\mathrm{CDCl}_{3}\left(\delta\right.$ 77.0) were used as internal standards for ${ }^{1} \mathrm{H}$ and ${ }^{13} \mathrm{C}$ NMR spectra respectively. Coupling constants $(J)$ are reported in Hertz $(\mathrm{Hz})$. The following abbreviations are used: s, singlet, d, doublet, t, triplet, q, quartet, h, heptulet, m, multiplet, br, broad.

Enantiomeric excesses (ee) were determined by SFC analyses using a Waters alliance e2695 system equipped with chiral stationary phase columns Daicel Chiralpak coupled with a dual wavelength $(215 / 254 \mathrm{~nm})$ Waters 2489 UV detector. Optical rotations were measured on a Perkin-Elmer 241 polarimeter $(\lambda=589 \mathrm{~nm}, \mathrm{Na}$ lamp, $1 \mathrm{dm}$ cell). High-resolution mass spectroscopic (HRMS) analyses were measured on LTQOrbitrap (Thermo Fisher Scientific) at Pierre et Marie Curie University. Melting points were measured with a Stuart Scientific melting point apparatus SMP1.

X-ray diffraction was made at Pierre et Marie Curie University. CCDC 1939901 and CCDC 1987761 contains the supplementary crystallographic data for compound $\mathbf{2 k}$ and $\mathbf{5 e}$ respectively. These data can be obtained free of charge from The Cambridge Crystallographic Data Centre via www.ccdc.cam.ac.uk/data_request/cif.

\section{Synthesis of starting materials}

\section{A. General procedure (1) for the preparation of starting materials}

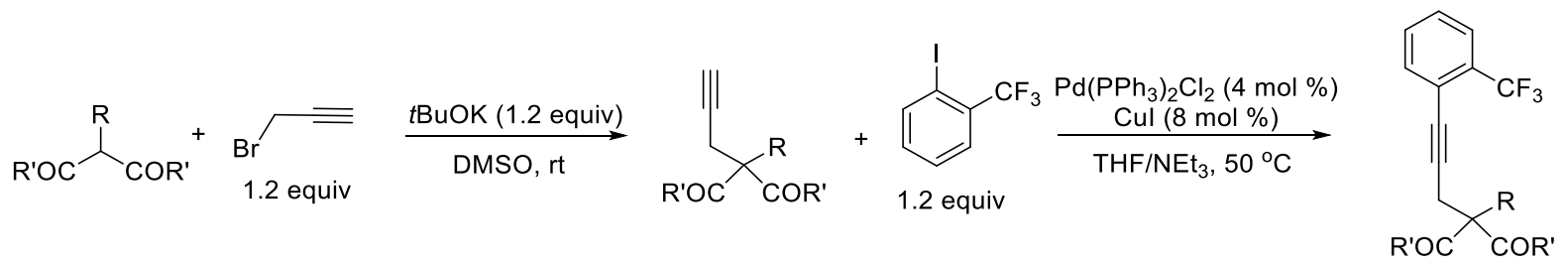

To a solution of 1,3-diketone ( 1 equiv) in dry DMSO was added $t \mathrm{BuOK}$ (1.2 equiv) under argon at room tempeature. The mixture was stirred at room temperature for $0.5 \mathrm{~h}$, then propargyl bromide (1.2 equiv) was added and the reaction was stirred at room temperature. After full conversion of the starting material, the mixture was diluted with aq. $\mathrm{HCl} 1 \mathrm{M}$ and extracted with ethyl acetate. Combined organic layers were washed with brine, dried over $\mathrm{MgSO}_{4}$, filtered and concentrated under vacuum. In a Schlenk tube, equipped with a magnetic stirring bar, were introduced the crude mixture, $\mathrm{PdCl}_{2}\left(\mathrm{PPh}_{3}\right)_{2}(4 \mathrm{~mol} \%)$, $\mathrm{CuI}(8 \mathrm{~mol} \%)$ and THF. The tube was evacuated under vacuum and placed under argon atmosphere. Then, 2-(trifluoromethyl)iodobenzene (1.2 equiv) and $\mathrm{Et}_{3} \mathrm{~N}$ were added successively under an argon atmosphere. The resulting mixture was stirred at $50{ }^{\circ} \mathrm{C}$ (oil bath) until full conversion. The reaction mixture was diluted with water and extracted with ethyl acetate. Combined organic 
layers were washed with brine, dried over $\mathrm{MgSO}_{4}$, filtered and concentrated under vacuum. The crude was purified by flash chromatography on silica gel.

2-methyl-2-(3-(2-(trifluoromethyl)phenyl)prop-2-yn-1-yl)cyclopentane-1,3-dione (1a)<smiles>CC1(CC#Cc2ccccc2C(F)(F)F)C(=O)CCC1=O</smiles>

Orange oil (3.2 g, $72 \%)$ obtained from the general procedure (1) using 2-methylcyclopentane1,3-dione (1.1 g, $5.3 \mathrm{mmol})$.

$\mathbf{R}_{\mathbf{f}}=0.31$ (petroleum ether/EtOAc 80:20).

${ }^{1} \mathbf{H}$ NMR $\left(400 \mathrm{MHz}, \mathrm{CDCl}_{3}, \delta\right): 7.61-7.56(\mathrm{~m}, 1 \mathrm{H}), 7.50-7.39(\mathrm{~m}, 2 \mathrm{H}), 7.39-7.33(\mathrm{~m}, 1 \mathrm{H})$, $2.83(\mathrm{~d}, J=1.8 \mathrm{~Hz}, 2 \mathrm{H}), 2.82(\mathrm{~d}, J=1.8 \mathrm{~Hz}, 2 \mathrm{H}), 2.75(\mathrm{~s}, 2 \mathrm{H}), 1.17(\mathrm{~s}, 3 \mathrm{H})$.

${ }^{13} \mathbf{C}$ NMR (101 MHz, $\mathrm{CDCl}_{3}, \delta$ ): $215.0(2 \mathrm{C}), 134.3,131.4,131.3$ (q, $\left.J=30.1 \mathrm{~Hz}\right), 128.0,125.7$ $(\mathrm{q}, J=4.9 \mathrm{~Hz}), 123.4(\mathrm{q}, J=273.1 \mathrm{~Hz}), 121.0(\mathrm{q}, J=1.9 \mathrm{~Hz}), 90.3,78.4,55.5,35.4(2 \mathrm{C}), 24.9$, 20.0 .

${ }^{19}$ F NMR $\left(376 \mathrm{MHz}, \mathrm{CDCl}_{3}, \delta\right):-62.2$.

HRMS (ESI): Calculated for $\mathrm{C}_{16} \mathrm{H}_{13} \mathrm{~F}_{3} \mathrm{O}_{2} \mathrm{Na}(\mathrm{M}+\mathrm{Na})^{+} 317.0765$, found 317.0771 .

2-methyl-2-(3-(2-(trifluoromethyl)phenyl)prop-2-yn-1-yl)cyclohexane-1,3-dione (1b)<smiles>CC1(CC#Cc2ccccc2C(F)(F)F)C(=O)CCCC1=O</smiles>

Orange oil (4.1 g, $46 \%)$ obtained from the general procedure (1) using 2-methylcyclohexane1,3-dione (3.5 g, $28 \mathrm{mmol})$.

$\mathbf{R}_{\mathbf{f}}=0.21$ (petroleum ether/EtOAc 80:20).

${ }^{1}$ H NMR (400 MHz, $\left.\mathrm{CDCl}_{3}, \delta\right): 7.57(\mathrm{~d}, J=7.5 \mathrm{~Hz}, 1 \mathrm{H}), 7.49(\mathrm{~d}, J=7.5 \mathrm{~Hz}, 1 \mathrm{H}), 7.42(\mathrm{t}, J=$ $7.5 \mathrm{~Hz}, 1 \mathrm{H}), 7.33(\mathrm{t}, J=7.5 \mathrm{~Hz}, 1 \mathrm{H}), 2.92(\mathrm{~s}, 2 \mathrm{H}), 2.83-2.59(\mathrm{~m}, 4 \mathrm{H}), 2.19-1.92(\mathrm{~m}, 2 \mathrm{H})$, $1.37(\mathrm{~s}, 1 \mathrm{H})$.

${ }^{13}$ C NMR (101 MHz, $\mathrm{CDCl}_{3}, \delta$ ): 208.7 (2C), 134.2, 131.3, 131.2 (q, $\left.J=30.4 \mathrm{~Hz}\right), 127.6,125.6$ $(\mathrm{q}, J=5.0 \mathrm{~Hz}), 123.5(\mathrm{q}, J=273.3 \mathrm{~Hz}), 121.5(\mathrm{q}, J=1.9 \mathrm{~Hz}), 92.1,78.4,64.3,37.9(2 \mathrm{C}), 25.3$, $22.8,17.2$.

${ }^{19}$ F NMR $\left(376 \mathrm{MHz}, \mathrm{CDCl}_{3}, \delta\right):-62.1$.

HRMS (ESI): Calculated for $\mathrm{C}_{17} \mathrm{H}_{15} \mathrm{~F}_{3} \mathrm{O}_{2} \mathrm{Na}(\mathrm{M}+\mathrm{Na})^{+} 331.0922$, found 331.0916.

2-benzyl-2-(3-(2-(trifluoromethyl)phenyl)prop-2-yn-1-yl)cyclohexane-1,3-dione (1c) 
<smiles>O=C1CCCC(=O)C1(CBr)CC#Cc1ccccc1C(F)(F)F</smiles>

Orange oil (751 mg, $37 \%$ ) obtained from the general procedure (1) using 2-benzylcyclohexane1,3-dione (1.1 g, $5.3 \mathrm{mmol})$.

$\mathbf{R}_{\mathbf{f}}=0.37$ (petroleum ether/EtOAc 80:20).

${ }^{1}$ H NMR (400 MHz, $\left.\mathrm{CDCl}_{3}, \delta\right): 7.66-7.58(\mathrm{~m}, 1 \mathrm{H}), 7.56-7.48(\mathrm{~m}, 2 \mathrm{H}), 7.48-7.43(\mathrm{~m}, 1 \mathrm{H})$, $7.42-7.31(\mathrm{~m}, 1 \mathrm{H}), 7.29-7.22(\mathrm{~m}, 2 \mathrm{H}), 7.12-7.00(\mathrm{~m}, 2 \mathrm{H}), 3.13(\mathrm{~s}, 2 \mathrm{H}), 3.03(\mathrm{~s}, 2 \mathrm{H}), 2.55$ (ddd, $J=17.2,8.6,4.9 \mathrm{~Hz}, 2 \mathrm{H}), 2.23(\mathrm{ddd}, J=17.2,8.6,4.9 \mathrm{~Hz}, 2 \mathrm{H}), 1.83-1.70(\mathrm{~m}, 1 \mathrm{H})$, $1.26-1.13(\mathrm{~m}, 1 \mathrm{H})$.

${ }^{13}$ C NMR (101 MHz, $\left.\mathrm{CDCl}_{3}, \delta\right): 210.7(2 \mathrm{C}), 135.5,134.4,131.3(\mathrm{q}, J=30.1 \mathrm{~Hz}), 131.2,129.9$ (2C), $128.7(2 \mathrm{C}), 127.9,127.4,25.7(\mathrm{q}, J=5.3 \mathrm{~Hz}), 123.5$ (q, $J=273.3 \mathrm{~Hz}), 121.2$ (q, $J=1.8$ $\mathrm{Hz}), 91.5,78.7,67.9,44.6,40.5$ (2C), 27.3, 15.5.

${ }^{19}$ F NMR $\left(376 \mathrm{MHz}, \mathrm{CDCl}_{3}, \delta\right):-62.1$.

HRMS (ESI): Calculated for $\mathrm{C}_{23} \mathrm{H}_{19} \mathrm{~F}_{3} \mathrm{O}_{2} \mathrm{Na}(\mathrm{M}+\mathrm{Na})^{+} 384.1337$, found 384.1328.

\section{B. General procedure (2) for the preparation of starting materials}

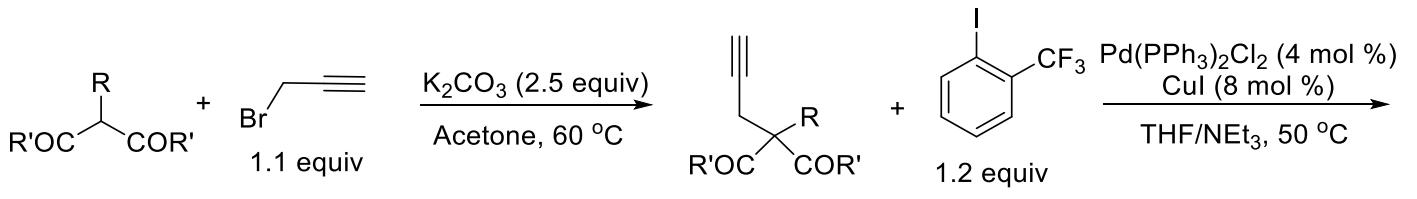<smiles>[R]C([R])([R])C([R])([R])CC#Cc1ccccc1C(F)(F)F</smiles>

To a solution of 1,3-diketone (1 equiv) in dry acetone was added $\mathrm{K}_{2} \mathrm{CO}_{3}$ (2.5 equiv) under argon at room tempeature. Then propargyl bromide (1.1 equiv) was added and the reaction was stirred at $60{ }^{\circ} \mathrm{C}$ (oil bath). After full conversion of the starting material, the mixture was diluted with aq. $\mathrm{HCl} 1 \mathrm{M}$ and extracted with ethyl acetate. Combined organic layers were washed with brine, dried over $\mathrm{MgSO}_{4}$, filtered and concentrated under vacuum. In a Schlenk tube, equipped with a magnetic stirring bar, were introduced the crude mixture, $\mathrm{PdCl}_{2}\left(\mathrm{PPh}_{3}\right)_{2}(4 \mathrm{~mol} \%), \mathrm{CuI}(8 \mathrm{~mol}$ $\%)$ and THF. The tube was evacuated under vacuum and placed under argon atmosphere. Then, 2-(trifluoromethyl)iodobenzene (1.2 equiv) and $\mathrm{Et}_{3} \mathrm{~N}$ were added successively under an argon atmosphere. The resulting mixture was stirred at $50{ }^{\circ} \mathrm{C}$ (oil bath) until full conversion. The reaction mixture was diluted with water and extracted with ethyl acetate. Combined organic layers were washed with brine, dried over $\mathrm{MgSO}_{4}$, filtered and concentrated under vacuum. The crude was purified by flash chromatography on silica gel.

3-Ethyl-3-(3-(2-(trifluoromethyl)phenyl)prop-2-yn-1-yl)pentane-2,4-dione (1d) 


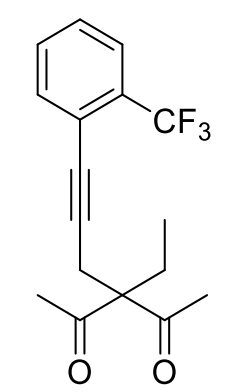

Orange oil (4.8 g, $67 \%$ ) obtained from the general procedure (2) using 3-ethylpentane-2,4dione (3 g, $23 \mathrm{mmol})$.

$\mathbf{R}_{\mathbf{f}}=0.42$ (petroleum ether/EtOAc 80:20).

${ }^{1} \mathbf{H}$ NMR $\left(400 \mathrm{MHz}, \mathrm{CDCl}_{3}, \delta\right): 7.61(\mathrm{~d}, J=7.8 \mathrm{~Hz}, 1 \mathrm{H}), 7.50(\mathrm{~d}, J=7.8 \mathrm{~Hz}, 1 \mathrm{H}), 7.45(\mathrm{t}, J=$ $7.8 \mathrm{~Hz}, 1 \mathrm{H}), 7.37$ (t, $J=7.8 \mathrm{~Hz}, 1 \mathrm{H}), 3.04(\mathrm{~s}, 2 \mathrm{H}), 2.21$ (q, $J=7,6 \mathrm{~Hz}, 2 \mathrm{H}), 2.18(\mathrm{~s}, 6 \mathrm{H}), 0.82$ (t, $J=7.6 \mathrm{~Hz}, 3 \mathrm{H})$.

${ }^{13}$ C NMR (101 MHz, $\left.\mathrm{CDCl}_{3}, \delta\right): 205.3$ (2C), 134.2, 131.35, 131.34 (q, $\left.J=30.4 \mathrm{~Hz}\right), 125.7$ (q, $J=5.3 \mathrm{~Hz}), 123.5(\mathrm{q}, J=273.4 \mathrm{~Hz}), 121.3(\mathrm{q}, J=2.0 \mathrm{~Hz}), 127.8,90.9,79.5,70.7,26.7(2 \mathrm{C})$, 23.7, 21.2, 8.1.

${ }^{19}$ F NMR $\left(376 \mathrm{MHz}, \mathrm{CDCl}_{3}, \delta\right):-62.4$.

HRMS (ESI): Calculated for $\mathrm{C}_{17} \mathrm{H}_{17} \mathrm{~F}_{3} \mathrm{O}_{2} \mathrm{Na}(\mathrm{M}+\mathrm{Na})^{+} 310.1181$, found 310.1188 .

2-(3-(2-(trifluoromethyl)phenyl)prop-2-yn-1-yl)isoindoline-1,3-dione (1e)

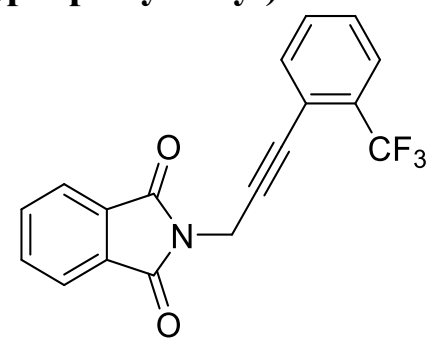

Orange oil ( $832 \mathrm{mg}, 55 \%$ ) obtained from the general procedure (2) using phthalimide (675 $\mathrm{mg}$, $4.6 \mathrm{mmol})$.

$\mathbf{R}_{\mathbf{f}}=0.10$ (petroleum ether/EtOAc 80:20).

${ }^{1} \mathbf{H}$ NMR (400 MHz, $\left.\mathrm{CDCl}_{3}, \delta\right): 7.89(\mathrm{dd}, J=5.5,3.1 \mathrm{~Hz}, 2 \mathrm{H}), 7.74(\mathrm{dd}, J=5.5,3.1 \mathrm{~Hz}, 2 \mathrm{H})$, $7.62-7.57(\mathrm{~m}, 1 \mathrm{H}), 7.57-7.51(\mathrm{~m}, 1 \mathrm{H}), 7.48-7.41(\mathrm{~m}, 1 \mathrm{H}), 7.41-7.32(\mathrm{~m}, 1 \mathrm{H}), 4.71(\mathrm{~s}$, $2 \mathrm{H})$.

${ }^{13}$ C NMR (101 MHz, $\mathrm{CDCl}_{3}, \delta$ ): 167.0 (2C), 134.20 (2C), 134.17 (2C), 132.0, 131.6 (q, $J=$ $30.6 \mathrm{~Hz}), 131.3,128.3,125.7(\mathrm{q}, J=4.9 \mathrm{~Hz}), 123.6(2 \mathrm{C}), 123.3(\mathrm{q}, J=273.5 \mathrm{~Hz}), 120.5$ (q, $J$ $=1.9 \mathrm{~Hz}), 88.4,79.1,27.9$.

${ }^{19}$ F NMR $\left(376 \mathrm{MHz}, \mathrm{CDCl}_{3}, \delta\right):-62.4$.

HRMS (ESI): Calculated for $\mathrm{C}_{18} \mathrm{H}_{10} \mathrm{NF}_{3} \mathrm{O}_{2} \mathrm{Na}(\mathrm{M}+\mathrm{Na})^{+} 329.0664$, found 329.0670 . 


\section{Access to tetralone derivatives}

\section{A. General procedure (3) for the preparation of racemic 1-tetralol derivatives}

In a septum-capped vial, equipped with a magnetic stirring bar, were introduced $\left.[\mathrm{Rh}(\mathrm{OH})(\mathrm{cod})]_{2} 1.5 \mathrm{~mol} \%, 2.25 \mu \mathrm{mol}, 1.1 \mathrm{mg}\right)$, the corresponding boronic acid $(0.30 \mathrm{mmol})$ and 1,3-diketone derivative $(0.15 \mathrm{mmol})$. The vial was closed, evacuated under vacuum and placed under argon atmosphere. Degassed $\mathrm{MeOH}$ was added and the resulting mixture was heated at $60{ }^{\circ} \mathrm{C}$ (preheated oil bath) until full consumption of the starting material. Solvents were removed under vacuum and the crude was purified by silica gel chromatography.

\section{B. General procedure (4) for the preparation of chiral 1-tetralol derivatives}

In a septum-capped vial, equipped with a magnetic stirring bar, were introduced the chiral diene 2-Me-4-MeOC $6 \mathrm{H}_{3}-\mathrm{MSBod}(3.3 \mathrm{~mol} \%, 10.0 \mu \mathrm{mol}, 3.4 \mathrm{mg})$ and $\left[\mathrm{RhCl}\left(\mathrm{C}_{2} \mathrm{H}_{4}\right)_{2}\right]_{2}(1.5 \mathrm{~mol} \%$, $4.5 \mu \mathrm{mol}, 1.8 \mathrm{mg}$ ). The vial was closed, evacuated under vacuum and placed under argon atmosphere. Degassed dichloromethane $(0.2 \mathrm{~mL})$ was added and the mixture was stirred for 15 min at room temperature. Then, a degassed solution of $\mathrm{KOH}$ in $\mathrm{MeOH}(50 \mu \mathrm{L}, \mathrm{c}=0.22 \mathrm{M})$ was added, the solution was stirred for $15 \mathrm{~min}$ and solvents were removed under vacuum. The corresponding boronic acid $(0.30 \mathrm{mmol})$ and the 1,3 -diketone derivative $(0.15 \mathrm{mmol})$ were added to the dry residue, followed by degassed methanol $(0.5 \mathrm{~mL})$ and the resulting mixture was heated at $60{ }^{\circ} \mathrm{C}$ (preheated oil bath) until full conversion of the starting material. Solvents were removed under vacuum and the crude was purified by silica gel chromatography.

\section{Characterization}

(E)-9b-hydroxy-3a-methyl-5-(2-(trifluoromethyl)benzylidene)-1,2,3a,4,5,9b-hexahydro3H-cyclopenta $[a]$ naphthalen-3-one (2a)

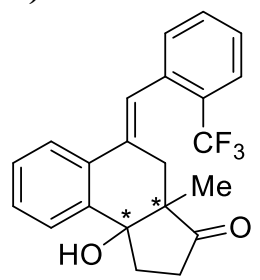

Yellow oil (52 mg, 93\%) obtained from the general procedure (4) using 1a (44 mg, $0.15 \mathrm{mmol}$ ) and phenylboronic acid (37 $\mathrm{mg}, 0.30 \mathrm{mmol})$.

$\mathbf{R}_{\mathbf{f}}=0.29$ (petroleum ether/EtOAc 80:20).

SFC (Chiralpak ID $s \mathrm{CO}_{2} / \mathrm{MeOH} 90: 10,2 \mathrm{~mL} / \mathrm{min}, \mathrm{P}=100$ bar): $\mathrm{Rt}=4.6 \mathrm{~min}$ (major), $5.5 \mathrm{~min}$ (minor).

$[\boldsymbol{\alpha}]_{\mathbf{D}}{ }^{\mathbf{2 0}}=+242\left(\mathrm{c}=2.3, \mathrm{CHCl}_{3}\right)$ for an enantiomeric excess of $84 \%$.

${ }^{1}$ H NMR $\left(400 \mathrm{MHz}, \mathrm{CDCl}_{3}, \delta\right): 7.73(\mathrm{dd}, J=7.8,1.5 \mathrm{~Hz}, 1 \mathrm{H}), 7.72-7.66(\mathrm{~m}, 2 \mathrm{H}), 7.55-7.48$ (m, 1H), $7.47-7.39(\mathrm{~m}, 2 \mathrm{H}), 7.37(\mathrm{dd}, J=7.8,1.5 \mathrm{~Hz}, 1 \mathrm{H}), 7.35-7.32(\mathrm{~m}, 1 \mathrm{H}), 7.26(\mathrm{~d}, J=$ $7.6 \mathrm{~Hz}, 1 \mathrm{H}), 2.69-2.56(\mathrm{~m}, 1 \mathrm{H}), 2.52(\mathrm{dd}, J=14.1,2.1 \mathrm{~Hz}, 1 \mathrm{H}), 2.49-2.38(\mathrm{~m}, 3 \mathrm{H}), 2.30(\mathrm{~m}$, $1 \mathrm{H}), 1.83(\mathrm{~d}, J=2.2 \mathrm{~Hz}, 1 \mathrm{H}), 1.01(\mathrm{~s}, 3 \mathrm{H})$. 
${ }^{13}$ C NMR (101 MHz, $\left.\mathrm{CDCl}_{3}, \delta\right): 220.1,140.4,135.9,134.2,133.5,131.7,131.1,129.3,129.0$ $(\mathrm{q}, J=29.5 \mathrm{~Hz}), 128.3,127.4,126.7,126.1(\mathrm{q}, J=5.2 \mathrm{~Hz}), 124.4,124.2(\mathrm{q}, J=273.9 \mathrm{~Hz})$, 124.1, 80.1, 54.3, 36.0, 35.3, 34.7, 13.9.

${ }^{19}$ F NMR (376 MHz, $\left.\mathrm{CDCl}_{3}, \delta\right):-60.6$.

HRMS (ESI): Calculated for $\mathrm{C}_{22} \mathrm{H}_{19} \mathrm{~F}_{3} \mathrm{O}_{2} \mathrm{Na}(\mathrm{M}+\mathrm{Na})^{+} 395.1235$, found 395.1231 .

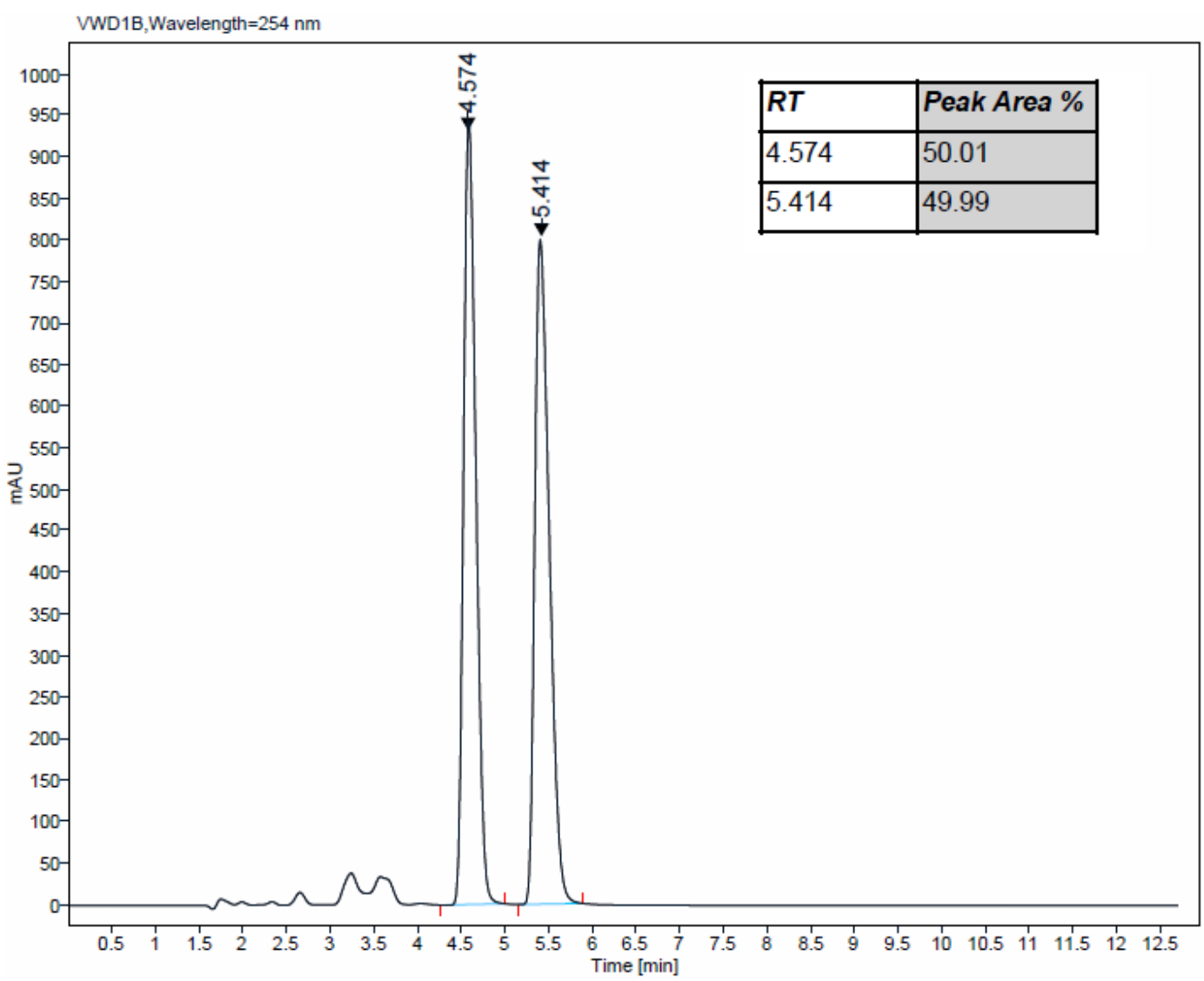




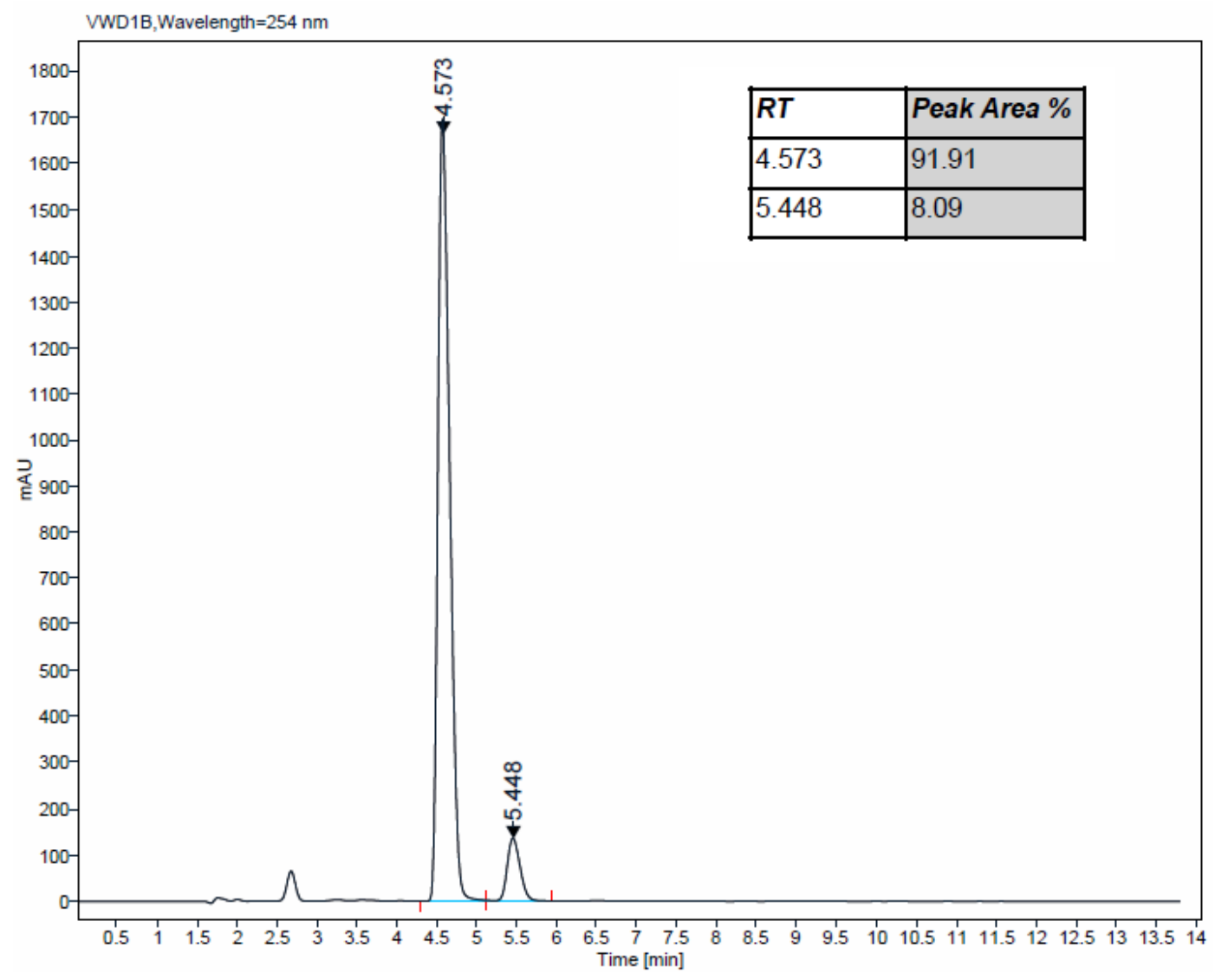

(E)-4a-hydroxy-10a-methyl-9-(2-(trifluoromethyl)benzylidene)-3,4,4a,9,10,10ahexahydrophenanthren-1(2H)-one (2b)

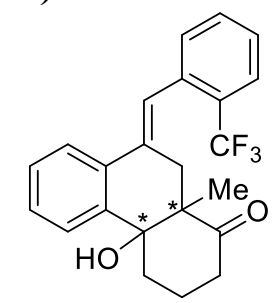

Orange oil (49 mg, 85\%) obtained from the general procedure (4) using $1 \mathbf{b}$ (46 mg, $0.15 \mathrm{mmol}$ ) and phenylboronic acid (37 $\mathrm{mg}, 0.30 \mathrm{mmol}$ ).

$\mathbf{R}_{\mathbf{f}}=0.22$ (petroleum ether/EtOAc 80:20).

SFC (Chiralpak ADH $s \mathrm{CO}_{2} / \mathrm{MeOH} 80: 20,2 \mathrm{~mL} / \mathrm{min}, \mathrm{P}=100$ bar): $\mathrm{Rt}=4.3 \mathrm{~min}$ (major), 7.5 $\min$ (minor).

$[\alpha]_{\mathbf{D}^{20}}=+88\left(\mathrm{c}=1.1, \mathrm{CHCl}_{3}\right)$ for an enantiomeric excess of $87 \%$.

${ }^{1}$ H NMR $\left(400 \mathrm{MHz}, \mathrm{CDCl}_{3}, \delta\right): 7.74(\mathrm{dd}, J=7.7,1.6 \mathrm{~Hz}, 1 \mathrm{H}), 7.70(\mathrm{~d}, J=7.7 \mathrm{~Hz}, 1 \mathrm{H}), 7.65$ $(\mathrm{dd}, J=7.7,1.6 \mathrm{~Hz}, 1 \mathrm{H}), 7.52(\mathrm{t}, J=7.7 \mathrm{~Hz}, 1 \mathrm{H}), 7.42-7.31(\mathrm{~m}, 4 \mathrm{H}), 7.28(\mathrm{~d}, J=8.0 \mathrm{~Hz}$, $1 \mathrm{H}), 3.02(\mathrm{dd}, J=14.8,2.5 \mathrm{~Hz}, 1 \mathrm{H}), 2.66-2.54(\mathrm{~m}, 1 \mathrm{H}), 2.44(\mathrm{dd}, J=14.8,0.8 \mathrm{~Hz}, 1 \mathrm{H}), 2.41$ $-2.33(\mathrm{~m}, 1 \mathrm{H}), 2.32-2.15(\mathrm{~m}, 2 \mathrm{H}), 2.02-1.95(\mathrm{~m}, 1 \mathrm{H}), 1.94(\mathrm{~d}, J=2.1 \mathrm{~Hz}, 1 \mathrm{H}), 1.91-1.84$ $(\mathrm{m}, 1 \mathrm{H}), 1.02(\mathrm{~s}, 3 \mathrm{H})$.

${ }^{13} \mathbf{C}$ NMR $\left(101 \mathrm{MHz}, \mathrm{CDCl}_{3}, \delta\right): 213.6,142.5,136.2,134.1,133.1,131.6,131.0,129.03,129.01$ $(\mathrm{q}, J=29.3 \mathrm{~Hz}), 127.9,127.3,126.1(\mathrm{q}, J=5.1 \mathrm{~Hz}), 125.7,124.2(\mathrm{q}, J=273.8 \mathrm{~Hz}), 123.9$, 123.4, 78.4, 53.4, 37.2, 37.0, 36.8, 20.9, 15.6.

${ }^{19}$ F NMR $\left(376 \mathrm{MHz}, \mathrm{CDCl}_{3}, \delta\right):-60.8$.

HRMS (ESI): Calculated for $\mathrm{C}_{23} \mathrm{H}_{21} \mathrm{~F}_{3} \mathrm{O}_{2} \mathrm{Na}(\mathrm{M}+\mathrm{Na})^{+} 409.1391$, found 409.1385 . 

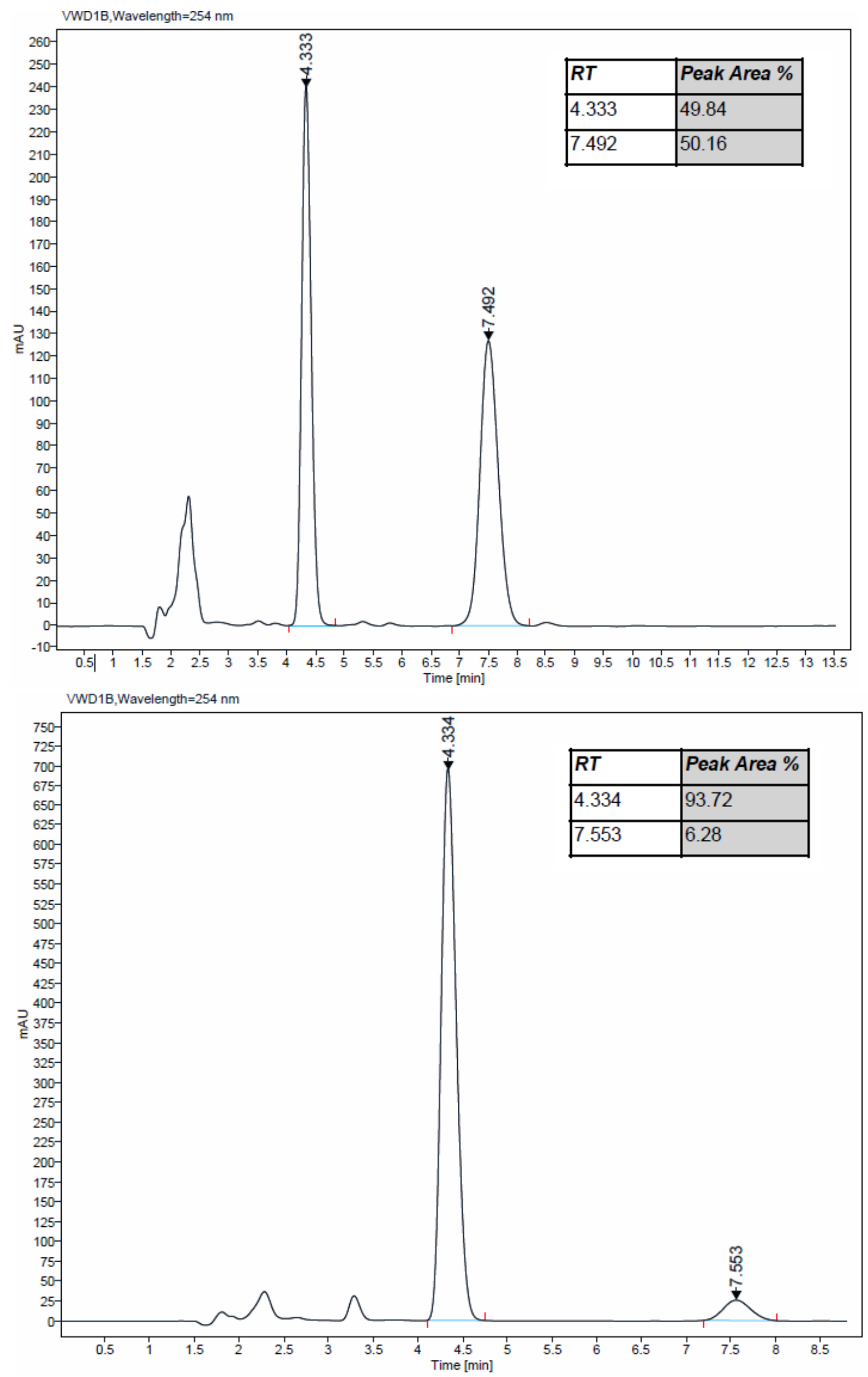

(E)-10a-benzyl-4a-hydroxy-9-(2-(trifluoromethyl)benzylidene)-3,4,4a,9,10,10ahexahydrophenanthren-1(2H)-one (2c) 
<smiles>O=C1CCCC2(O)C1(O)C/C(=C/c1ccccc1F)c1ccccc1C2(Br)Br</smiles>

Orange oil (58 mg, 84\%) obtained from the general procedure (4) using 1c (58 $\mathrm{mg}, 0.15 \mathrm{mmol}$ ) and phenylboronic acid (37 $\mathrm{mg}, 0.30 \mathrm{mmol})$.

$\mathbf{R}_{\mathbf{f}}=0.36$ (petroleum ether/EtOAc 80:20).

SFC (Chiralpak ADH $s \mathrm{CO}_{2} / \mathrm{MeOH} 90: 10,2 \mathrm{~mL} / \mathrm{min}, \mathrm{P}=100 \mathrm{bar}$ ): $\mathrm{Rt}=14.0 \mathrm{~min}$ (major), 17.7 $\min$ (minor).

$[\boldsymbol{\alpha}]_{\mathbf{D}}{ }^{\mathbf{2 0}}=-234\left(\mathrm{c}=0.8, \mathrm{CHCl}_{3}\right)$ for an enantiomeric excess of $90 \%$.

${ }^{1}$ H NMR $\left(400 \mathrm{MHz}, \mathrm{CDCl}_{3}, \delta\right): 7.90-7.80(\mathrm{~m}, 1 \mathrm{H}), 7.68-7.62(\mathrm{~m}, 1 \mathrm{H}), 7.62-7.57(\mathrm{~m}, 1 \mathrm{H})$, $7.43-7.38(\mathrm{~m}, 2 \mathrm{H}), 7.34-7.30(\mathrm{~m}, 3 \mathrm{H}), 7.16(\mathrm{~m}, 3 \mathrm{H}), 7.03-6.96(\mathrm{~m}, 2 \mathrm{H}), 6.94-6.89(\mathrm{~m}$, $1 \mathrm{H}), 3.10(\mathrm{~d}, J=13.7 \mathrm{~Hz}, 1 \mathrm{H}), 2.87(\mathrm{dd}, J=17.1,2.4 \mathrm{~Hz}, 1 \mathrm{H}), 2.81(\mathrm{~d}, J=13.7 \mathrm{~Hz}, 1 \mathrm{H}), 2.59$ (m, 1H), $2.50-2.44(\mathrm{~m}, 1 \mathrm{H}), 2.41$ (dd, $J=17.1,1.9 \mathrm{~Hz}, 1 \mathrm{H}), 2.26-2.11(\mathrm{~m}, 2 \mathrm{H}), 1.93(\mathrm{~d}, J$ $=1.9 \mathrm{~Hz}, 1 \mathrm{H}), 1.92-1.87(\mathrm{~m}, 1 \mathrm{H}), 1.82-1.73(\mathrm{~m}, 1 \mathrm{H})$.

${ }^{13} \mathrm{C}$ NMR $\left(101 \mathrm{MHz}, \mathrm{CDCl}_{3}, \delta\right): 212.7,141.8,138.4,136.3,133.5,133.2,131.6,131.1(2 \mathrm{C})$, $130.7,128.7,128.6(\mathrm{q}, J=29.5 \mathrm{~Hz}), 128.3(2 \mathrm{C}), 128.0,127.0,126.5,125.9(\mathrm{q}, J=5.7 \mathrm{~Hz})$, $124.8,124.2(\mathrm{q}, J=273.6 \mathrm{~Hz}), 124.0,123.0,79.3,57.9,37.0,36.2,35.7,33.2,20.5$.

${ }^{19}$ F NMR $\left(376 \mathrm{MHz}, \mathrm{CDCl}_{3}, \delta\right):-61.2$.

HRMS (ESI): Calculated for $\mathrm{C}_{29} \mathrm{H}_{25} \mathrm{~F}_{3} \mathrm{O}_{2} \mathrm{Na}(\mathrm{M}+\mathrm{Na})^{+} 485.1704$, found 485.1699 .

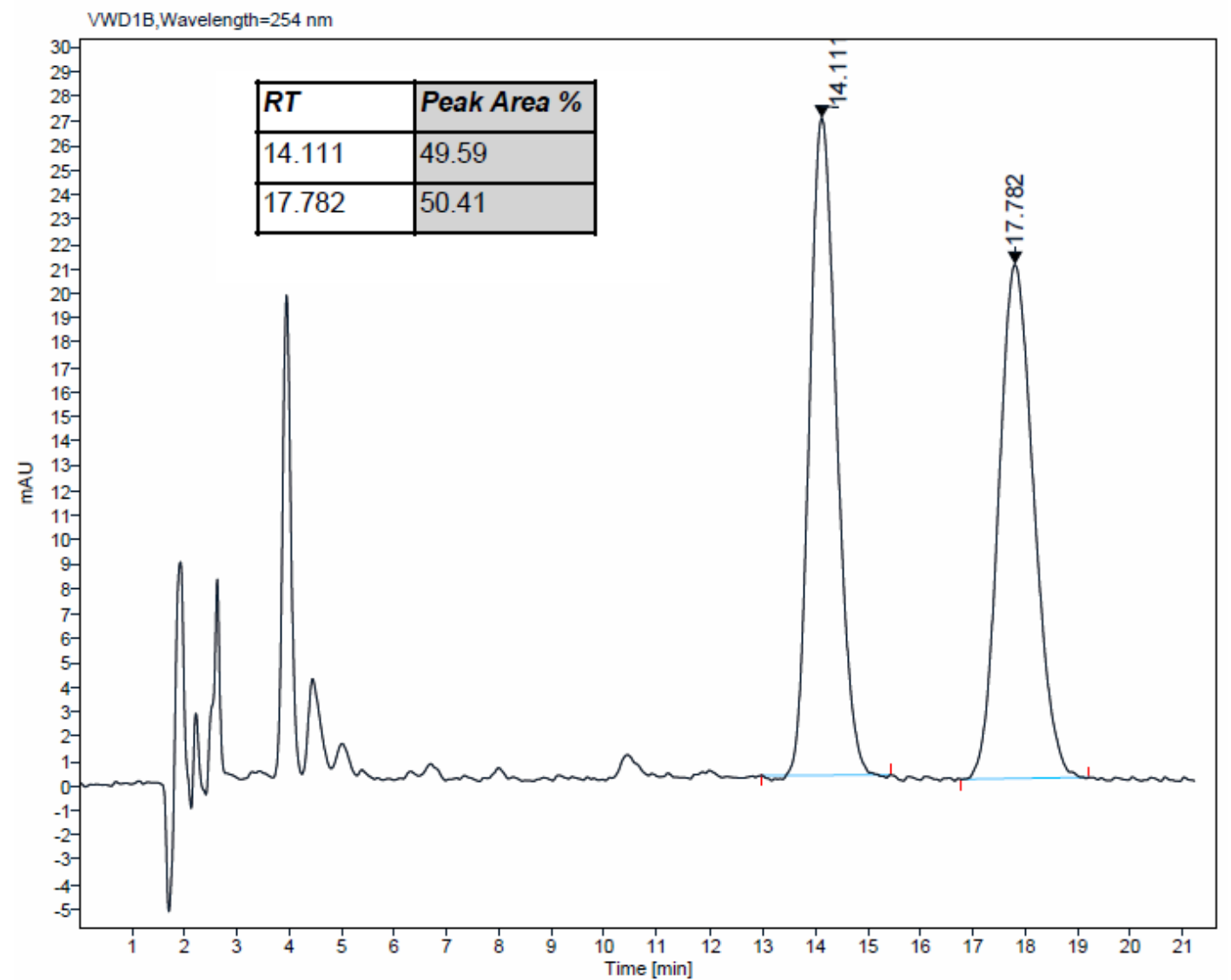




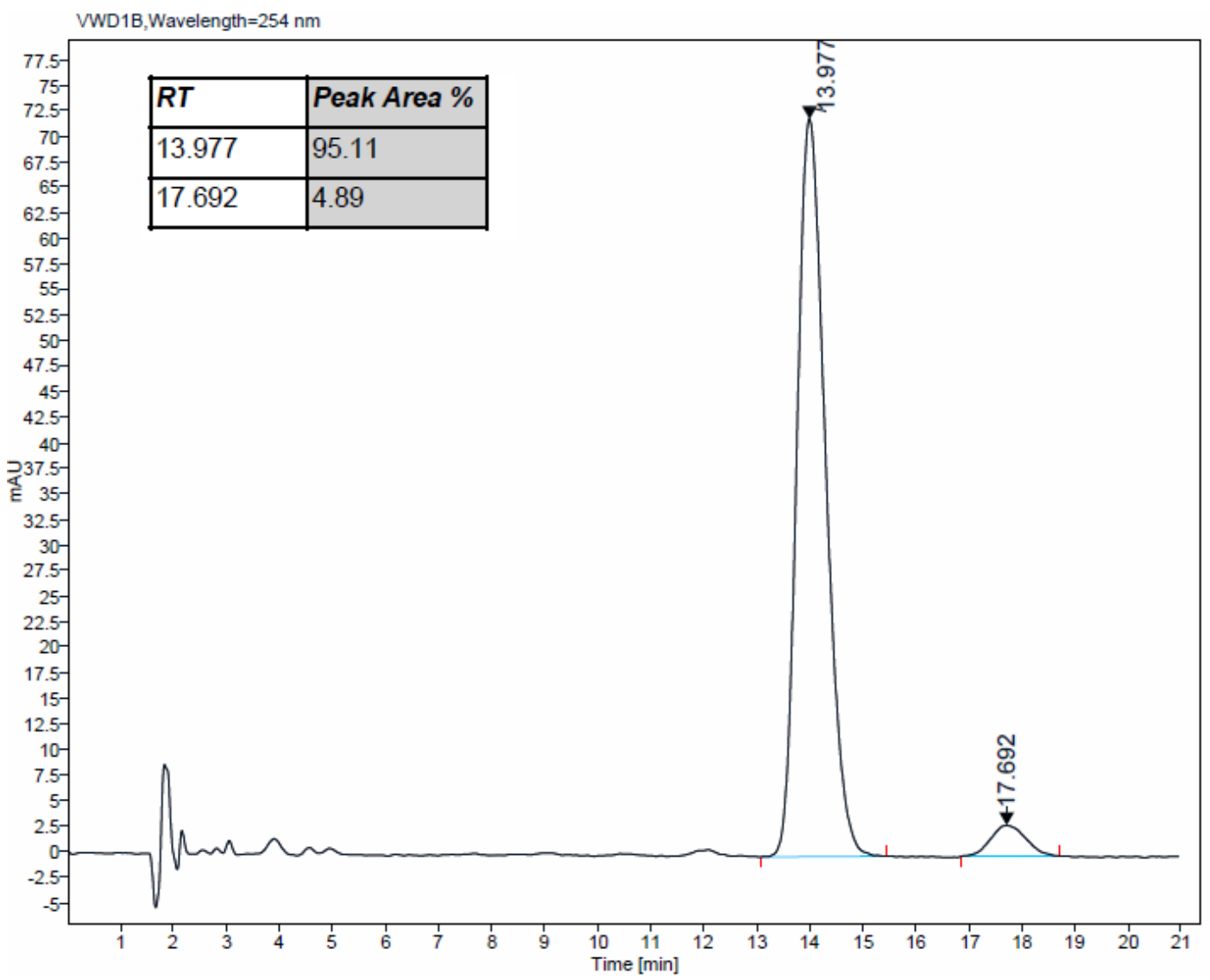

(E)-6a-benzyl-11b-hydroxy-5-(2-(trifluoromethyl)benzylidene)-5,6,6a,11b-tetrahydro7H-benzo[c]fluoren-7-one (2d)<smiles>CC1(Br)C(=O)c2ccccc2C1(Br)Br</smiles>

Yellow oil (61 mg, 82\%) obtained from the general procedure (4).

$\mathbf{R}_{\mathbf{f}}=0.24$ (petroleum ether/EtOAc 80:20).

SFC (Chiralpak ADH $s \mathrm{CO}_{2} / \mathrm{MeOH} 80: 20,2 \mathrm{~mL} / \mathrm{min}, \mathrm{P}=100 \mathrm{bar}$ ): $\mathrm{Rt}=6.6 \mathrm{~min}$ (minor), 14.5 $\min$ (major).

$[\boldsymbol{\alpha}]_{\mathbf{D}^{\mathbf{2 0}}}=+185\left(\mathrm{c}=2.3, \mathrm{CHCl}_{3}\right)$ for an enantiomeric excess of $74 \%$.

${ }^{1} \mathbf{H}$ NMR $\left(400 \mathrm{MHz}, \mathrm{CDCl}_{3}, \delta\right): 8.02(\mathrm{dd}, J=8.0,1.4 \mathrm{~Hz}, 1 \mathrm{H}), 7.79(\mathrm{dt}, J=7.8,0.9 \mathrm{~Hz}, 1 \mathrm{H})$, $7.69(\mathrm{~d}, J=7.9 \mathrm{~Hz}, 1 \mathrm{H}), 7.66-7.45(\mathrm{~m}, 5 \mathrm{H}), 7.45-7.27(\mathrm{~m}, 4 \mathrm{H}), 7.13-6.95(\mathrm{~m}, 6 \mathrm{H}), 3.28$ $(\mathrm{d}, J=13.9 \mathrm{~Hz}, 1 \mathrm{H}), 3.18(\mathrm{~d}, J=13.8 \mathrm{~Hz}, 1 \mathrm{H}), 3.02(\mathrm{~d}, J=13.8 \mathrm{~Hz}, 1 \mathrm{H}), 2.64(\mathrm{~s}, 1 \mathrm{H}), 2.41$ $(\mathrm{dd}, J=13.9,2.0 \mathrm{~Hz}, 1 \mathrm{H})$.

${ }^{13}$ C NMR (101 MHz, $\left.\mathrm{CDCl}_{3}, \delta\right)$ :

205.2, 157.2, 140.7, 136.9, 135.9, 135.8, 135.4 (2C), 134.1, 131.5, 131.2, 130.6 (2C), 129.2, $129.0(\mathrm{q}, J=29.5 \mathrm{~Hz}), 128.9,128.2,127.9(2 \mathrm{C}), 127.4,127.0,126.5,126.1(\mathrm{q}, J=5.2 \mathrm{~Hz})$, $125.4,124.5,124.2(\mathrm{q}, J=273.9 \mathrm{~Hz}), 123.2,123.0,79.0,61.0,41.7,32.0$.

${ }^{19}$ F NMR $\left(376 \mathrm{MHz}, \mathrm{CDCl}_{3}, \delta\right):-60.6$.

HRMS (ESI): Calculated for $\mathrm{C}_{32} \mathrm{H}_{23} \mathrm{~F}_{3} \mathrm{O}_{2} \mathrm{Na}(\mathrm{M}+\mathrm{Na})^{+}$519.1548, found 519.1556. 

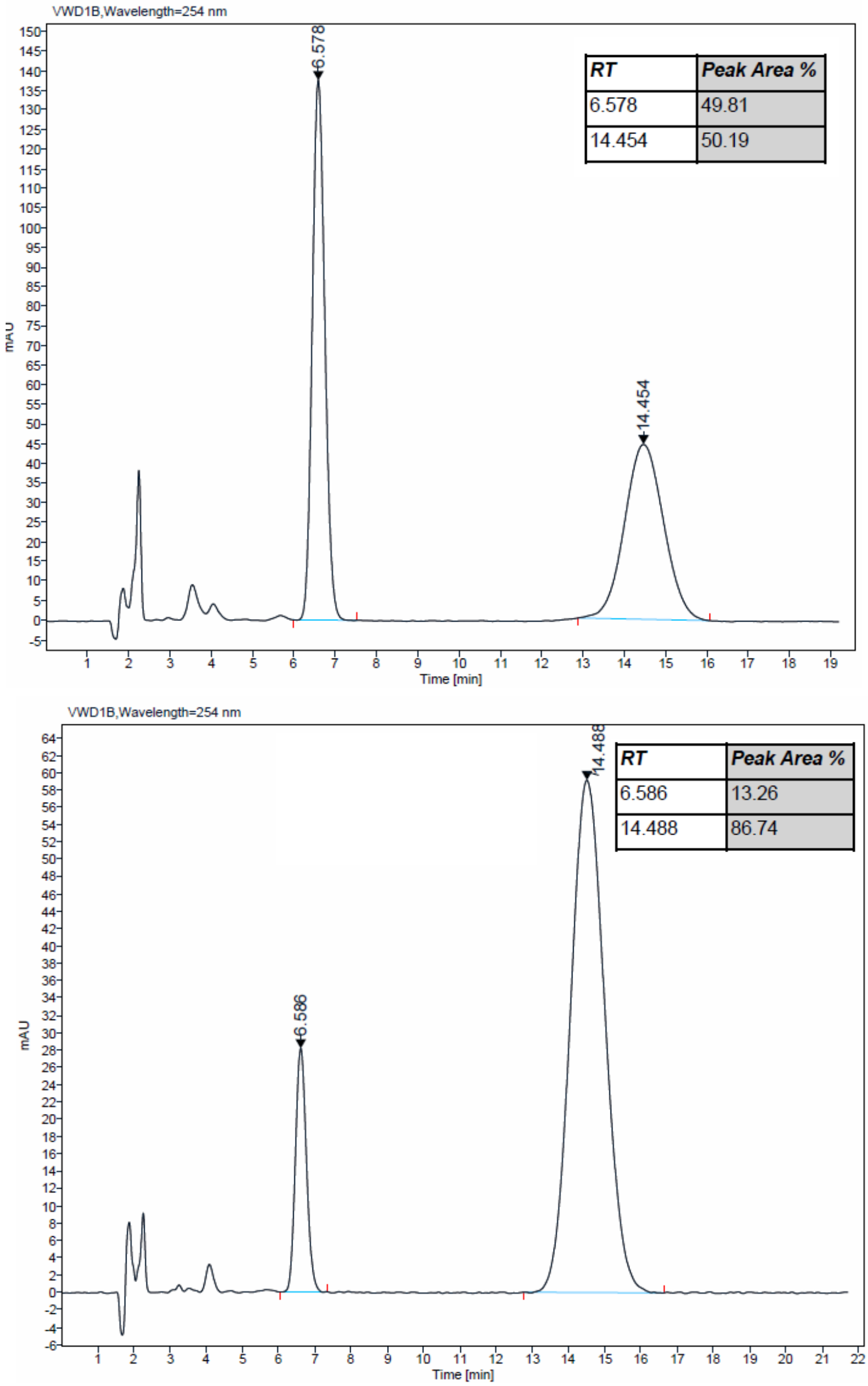

(E)-1-(2-ethyl-1-hydroxy-1-methyl-4-(2-(trifluoromethyl)benzylidene)-1,2,3,4tetrahydronaphthalen-2-yl)ethan-1-one (2e) 


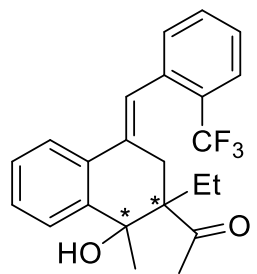

Colorless oil (48 mg, 83\%) obtained from the general procedure (4) using 1d (47 mg, 0.15 $\mathrm{mmol})$ and phenylboronic acid (37 $\mathrm{mg}, 0.30 \mathrm{mmol})$.

$\mathbf{R}_{\mathbf{f}}=0.48$ (petroleum ether/EtOAc 80:20).

SFC (Chiralpak ADH $s \mathrm{CO}_{2} / \mathrm{MeOH} 90: 10,2 \mathrm{~mL} / \mathrm{min}, \mathrm{P}=100$ bar): $\mathrm{Rt}=3.4 \mathrm{~min}$ (major), 4.3 $\min$ (minor).

$[\alpha]_{\mathbf{D}}{ }^{\mathbf{2 0}}=+42\left(\mathrm{c}=1.5, \mathrm{CHCl}_{3}\right)$ for an enantiomeric excess of $80 \%$.

${ }^{1}$ H NMR (400 MHz, $\left.\mathrm{CDCl}_{3}, \delta\right): 7.79-7.72(\mathrm{~m}, 2 \mathrm{H}), 7.66(\mathrm{dd}, J=7.5,1.3 \mathrm{~Hz}, 1 \mathrm{H}), 7.60$ (t, $J$ $=7.5 \mathrm{~Hz}, 1 \mathrm{H}), 7.50-7.40(\mathrm{~m}, 2 \mathrm{H}), 7.34(\mathrm{td}, J=7.5,1.3 \mathrm{~Hz}, 1 \mathrm{H}), 7.31-7.29(\mathrm{~m}, 1 \mathrm{H}), 7.28-$ $7.23(\mathrm{~m}, 1 \mathrm{H}), 4.80(\mathrm{q}, J=1.3 \mathrm{~Hz}, 1 \mathrm{H}), 3.16(\mathrm{dd}, J=17.7,1.7 \mathrm{~Hz}, 1 \mathrm{H}), 2.65(\mathrm{dd}, J=17.7,2.8$ $\mathrm{Hz}, 1 \mathrm{H}), 2.26-2.13(\mathrm{~m}, 1 \mathrm{H}), 1.89(\mathrm{~s}, 3 \mathrm{H}), 1.66-1.52(\mathrm{~m}, 1 \mathrm{H}), 1.38(\mathrm{~d}, J=1.3 \mathrm{~Hz}, 3 \mathrm{H}), 0.87$ $(\mathrm{t}, J=7.6 \mathrm{~Hz}, 3 \mathrm{H})$.

${ }^{13}$ C NMR (101 MHz, $\left.\mathrm{CDCl}_{3}, \delta\right): 218.8,146.1,136.3,134.7,132.7,131.7,130.8,128.9,128.8$ $(\mathrm{q}, J=32.9 \mathrm{~Hz}), 127.4,127.3,126.3(\mathrm{q}, J=5.0 \mathrm{~Hz}), 124.8,124.2(\mathrm{q}, J=273.6 \mathrm{~Hz}), 123.7$, $122.3,75.7,57.0,34.5,29.4,27.8,25.8,9.8$.

${ }^{19}$ F NMR (376 MHz, $\left.\mathrm{CDCl}_{3}, \delta\right):-61.0$.

HRMS (ESI): Calculated for $\mathrm{C}_{23} \mathrm{H}_{23} \mathrm{~F}_{3} \mathrm{O}_{2} \mathrm{Na}(\mathrm{M}+\mathrm{Na})^{+} 411.1548$, found 411.1544 .

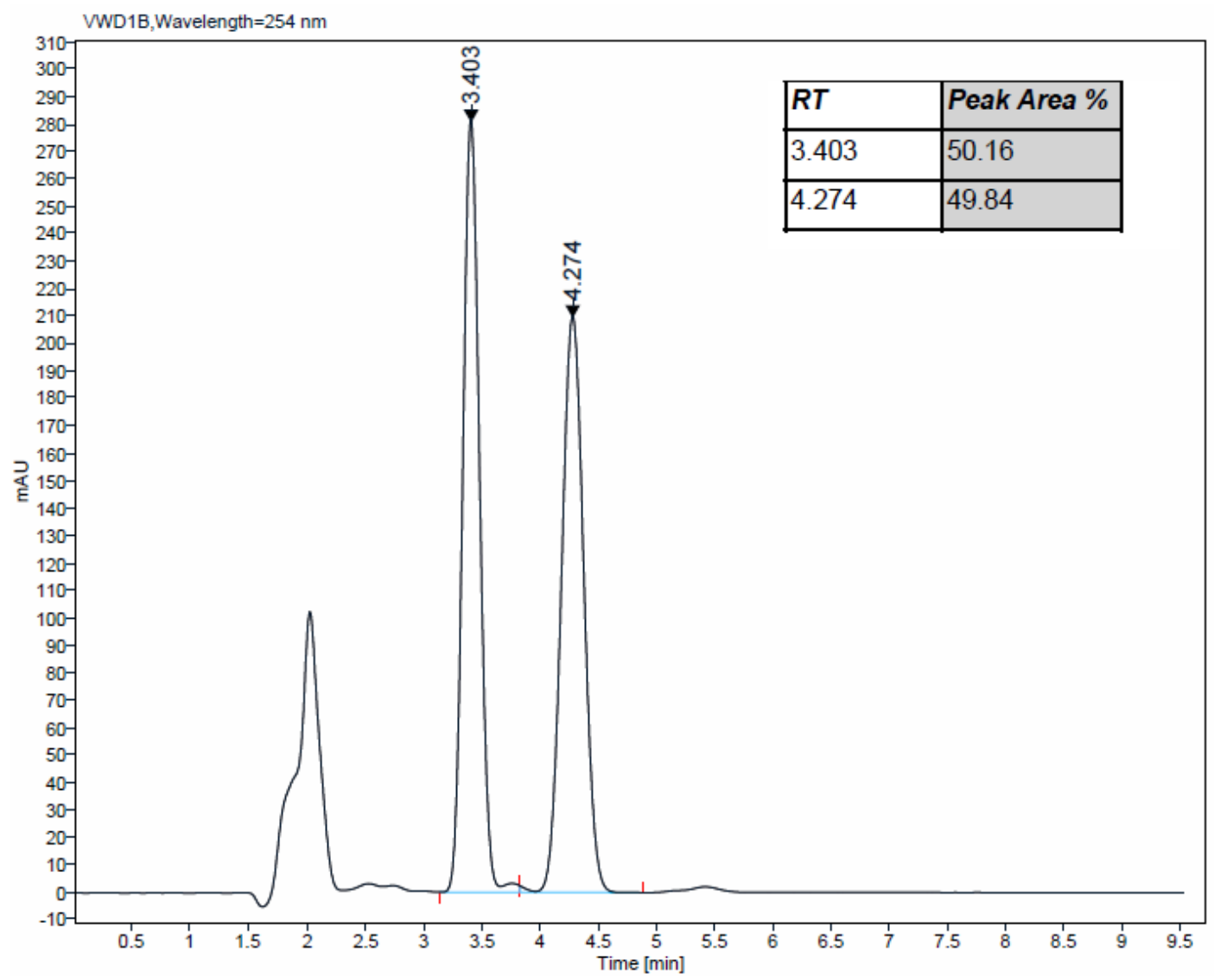




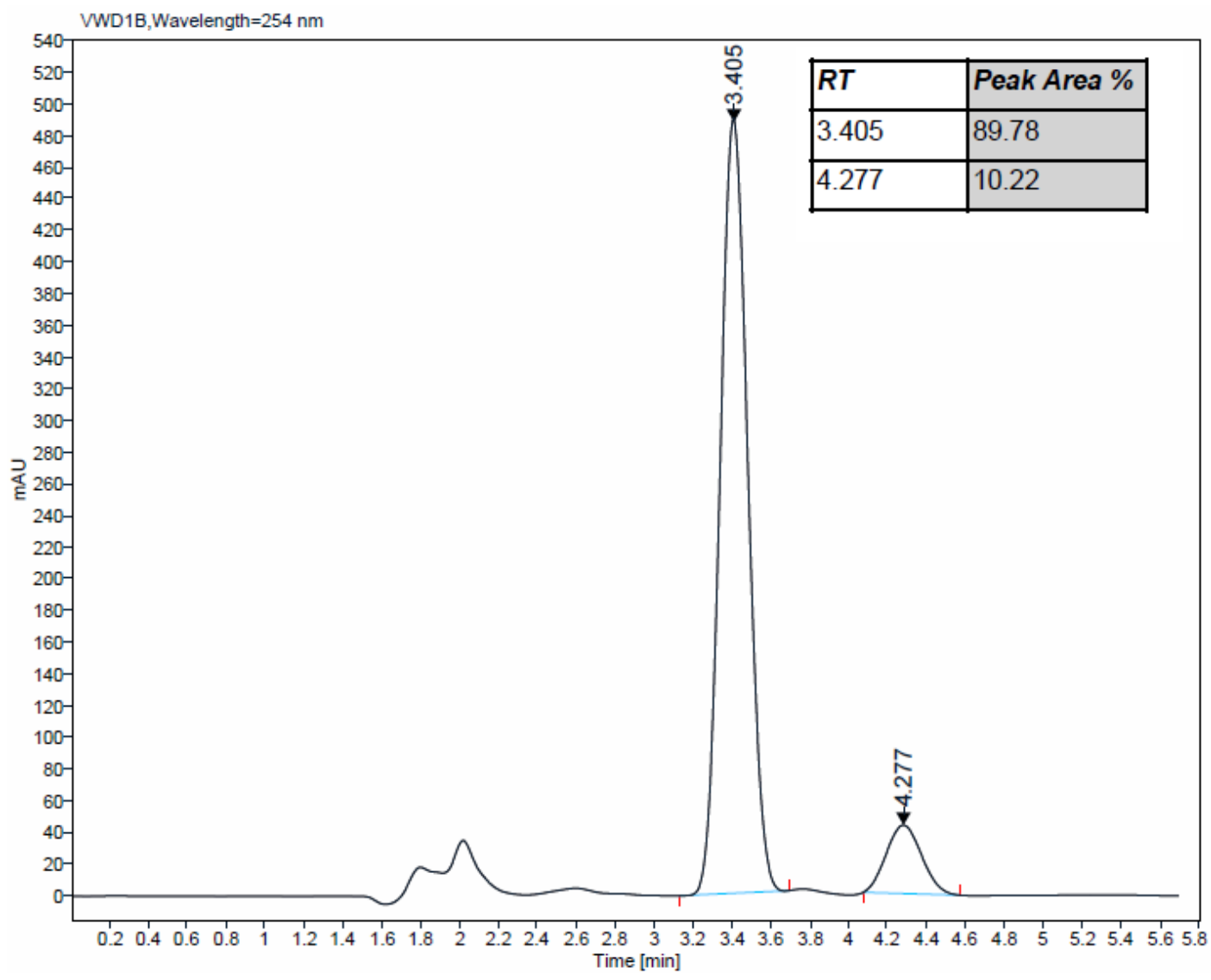

(E)-9b-hydroxy-3a,8-dimethyl-5-(2-(trifluoromethyl)benzylidene)-1,2,3a,4,5,9bhexahydro-3H-cyclopenta[a]naphthalen-3-one (2f)

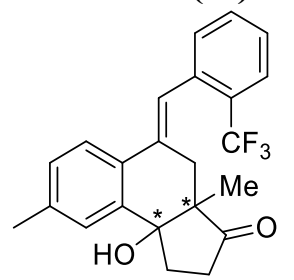

Yellow oil (55 mg, 96\%) obtained from the general procedure (4) using 1a (44 mg, $0.15 \mathrm{mmol}$ ) and 4-tolylboronic acid (41 $\mathrm{mg}, 0.30 \mathrm{mmol})$.

$\mathbf{R}_{\mathbf{f}}=0.25$ (petroleum ether/EtOAc 80:20).

SFC (Chiralpak ID $s \mathrm{CO}_{2} / \mathrm{MeOH} 90: 10,2 \mathrm{~mL} / \mathrm{min}, \mathrm{P}=100$ bar): $\mathrm{Rt}=4.5 \mathrm{~min}$ (major), $5.2 \mathrm{~min}$ (minor).

$[\boldsymbol{\alpha}]_{\mathbf{D}}{ }^{\mathbf{2 0}}=+301\left(\mathrm{c}=2.6, \mathrm{CHCl}_{3}\right)$ for an enantiomeric excess of $82 \%$.

${ }^{1} \mathbf{H}$ NMR $\left(400 \mathrm{MHz}, \mathrm{CDCl}_{3}, \delta\right): 7.69(\mathrm{~d}, J=7.8 \mathrm{~Hz}, 1 \mathrm{H}), 7.58(\mathrm{~d}, J=8.1 \mathrm{~Hz}, 1 \mathrm{H}), 7.55-7.47$ $(\mathrm{m}, 2 \mathrm{H}), 7.39$ (t, $J=7.8 \mathrm{~Hz}, 1 \mathrm{H}), 7.29(\mathrm{t}, J=2.4 \mathrm{~Hz}, 1 \mathrm{H}), 7.27-7.23(\mathrm{~m}, 1 \mathrm{H}), 7.17$ (dd, $J=$ 8.1, 1.9 Hz, 1H), $2.75-2.54(\mathrm{~m}, 1 \mathrm{H}), 2.54-2.35(\mathrm{~m}, 4 \mathrm{H}), 2.42(\mathrm{~s}, 3 \mathrm{H}), 2.35-2.22(\mathrm{~m}, 1 \mathrm{H})$, $1.80(\mathrm{~d}, J=2.2 \mathrm{~Hz}, 1 \mathrm{H}), 0.99(\mathrm{~s}, 3 \mathrm{H})$.

${ }^{13}$ C NMR (101 MHz, $\left.\mathrm{CDCl}_{3}, \delta\right): 220.1,140.2,139.3,136.0,134.1,131.7,131.2,130.8,129.3$, $129.0(\mathrm{q}, J=29.3 \mathrm{~Hz}), 127.2,127.0,126.0(\mathrm{q}, J=5.6 \mathrm{~Hz}), 124.4,124.3(\mathrm{q}, J=273.8 \mathrm{~Hz})$, $123.2,80.1,54.3,35.9,35.3,34.7,21.4,13.8$.

${ }^{19}$ F NMR $\left(376 \mathrm{MHz}, \mathrm{CDCl}_{3}, \delta\right):-60.5$.

HRMS (ESI): Calculated for $\mathrm{C}_{23} \mathrm{H}_{21} \mathrm{~F}_{3} \mathrm{O}_{2} \mathrm{Na}(\mathrm{M}+\mathrm{Na})^{+} 409.1391$, found 409.1387 . 

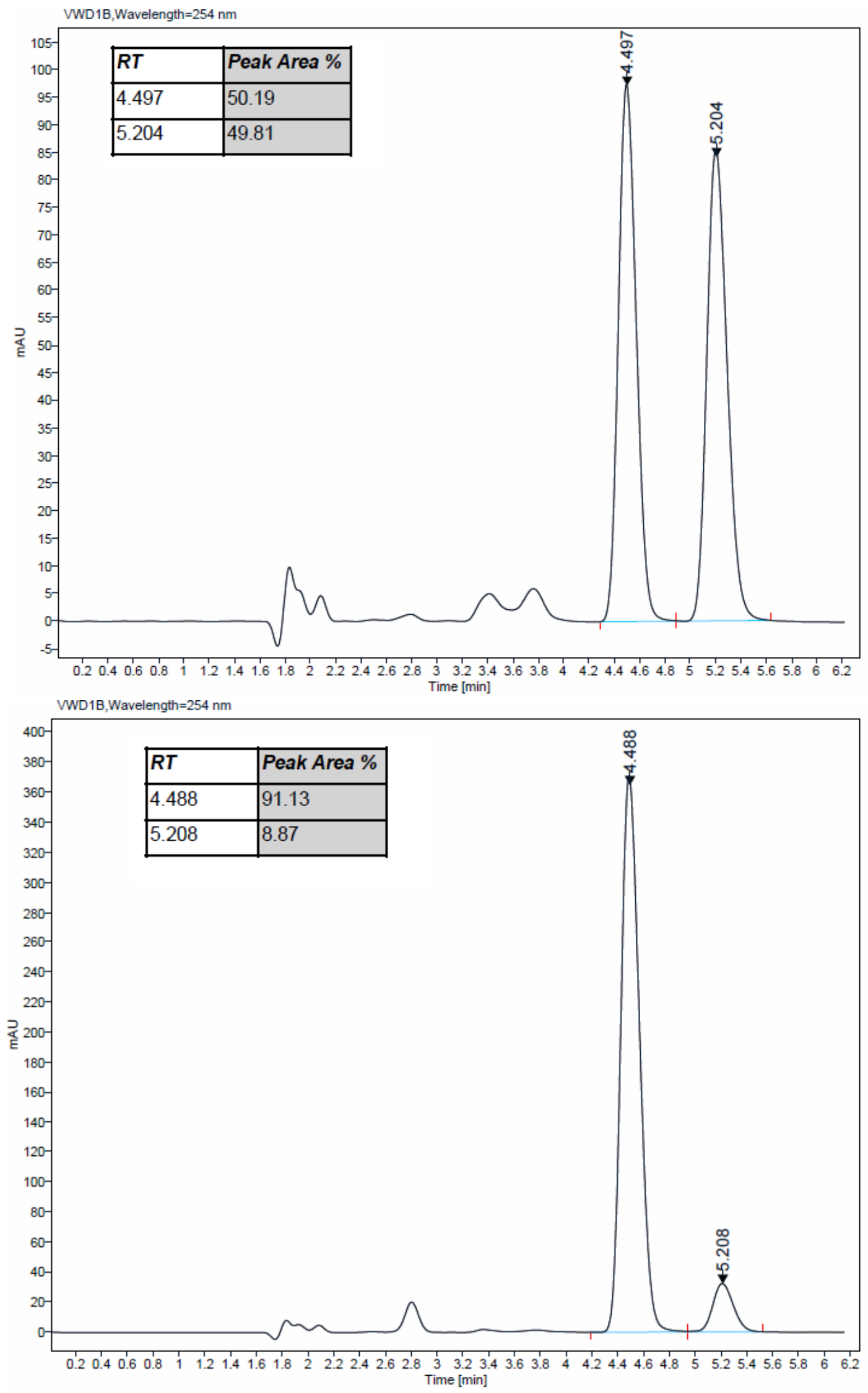

(E)-8-(tert-butyl)-9b-hydroxy-3a-methyl-5-(2-(trifluoromethyl)benzylidene)1,2,3a,4,5,9b-hexahydro-3H-cyclopenta[a]naphthalen-3-one (2g) 


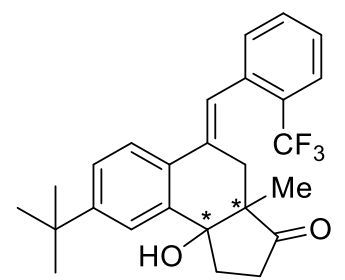

Colorless oil (68 mg, 99\%) obtained from the general procedure (4) using 1a (44 mg, 0.15 $\mathrm{mmol}$ ) and 4-tert-butylphenylboronic acid (53 $\mathrm{mg}, 0.30 \mathrm{mmol})$.

$\mathbf{R}_{\mathbf{f}}=0.34$ (petroleum ether/EtOAc 80:20).

SFC (Chiralpak ADH $s \mathrm{CO}_{2} / \mathrm{MeOH} 90: 10,2 \mathrm{~mL} / \mathrm{min}, \mathrm{P}=100$ bar): $\mathrm{Rt}=5.2 \mathrm{~min}$ (major), 7.7 $\min$ (minor).

$[\boldsymbol{\alpha}]_{\mathbf{D}}{ }^{\mathbf{2 0}}=+323\left(\mathrm{c}=2.0, \mathrm{CHCl}_{3}\right)$ for an enantiomeric excess of $85 \%$.

${ }^{1}$ H NMR $\left(400 \mathrm{MHz}, \mathrm{CDCl}_{3}, \delta\right): 7.74(\mathrm{~d}, J=2.1 \mathrm{~Hz}, 1 \mathrm{H}), 7.71-7.68(\mathrm{~m}, 1 \mathrm{H}), 7.63(\mathrm{~d}, J=8.4$ $\mathrm{Hz}, 1 \mathrm{H}), 7.55-7.46(\mathrm{~m}, 1 \mathrm{H}), 7.44-7.34(\mathrm{~m}, 2 \mathrm{H}), 7.32(\mathrm{t}, J=2.4 \mathrm{~Hz}, 1 \mathrm{H}), 7.25-7.21$ (m, $1 \mathrm{H}), 2.75-2.56(\mathrm{~m}, 1 \mathrm{H}), 2.54-2.38(\mathrm{~m}, 4 \mathrm{H}), 2.36-2.25(\mathrm{~m}, 1 \mathrm{H}), 1.83(\mathrm{~d}, J=2.3 \mathrm{~Hz}, 1 \mathrm{H})$, $1.37(\mathrm{~s}, 9 \mathrm{H}), 1.01(\mathrm{~s}, 3 \mathrm{H})$.

${ }^{13} \mathbf{C}$ NMR (101 MHz, $\left.\mathrm{CDCl}_{3}, \delta\right): 220.2,152.6,140.0,136.1,134.0,131.7,131.2,130.7,129.0$ $(\mathrm{q}, J=29.5 \mathrm{~Hz}), 127.2,126.0(\mathrm{q}, J=5.3 \mathrm{~Hz}), 125.6,124.2,123.3,123.1,121.5(\mathrm{q}, J=273.8$ $\mathrm{Hz}), 80.4,54.4,36.0,35.3,34.9,34.7,31.3(3 \mathrm{C}), 13.9$.

${ }^{19}$ F NMR $\left(376 \mathrm{MHz}, \mathrm{CDCl}_{3}, \delta\right):-60.6$.

HRMS (ESI): Calculated for $\mathrm{C}_{26} \mathrm{H}_{27} \mathrm{~F}_{3} \mathrm{O}_{2} \mathrm{Na}(\mathrm{M}+\mathrm{Na})^{+} 451.1861$, found 451.1855 .

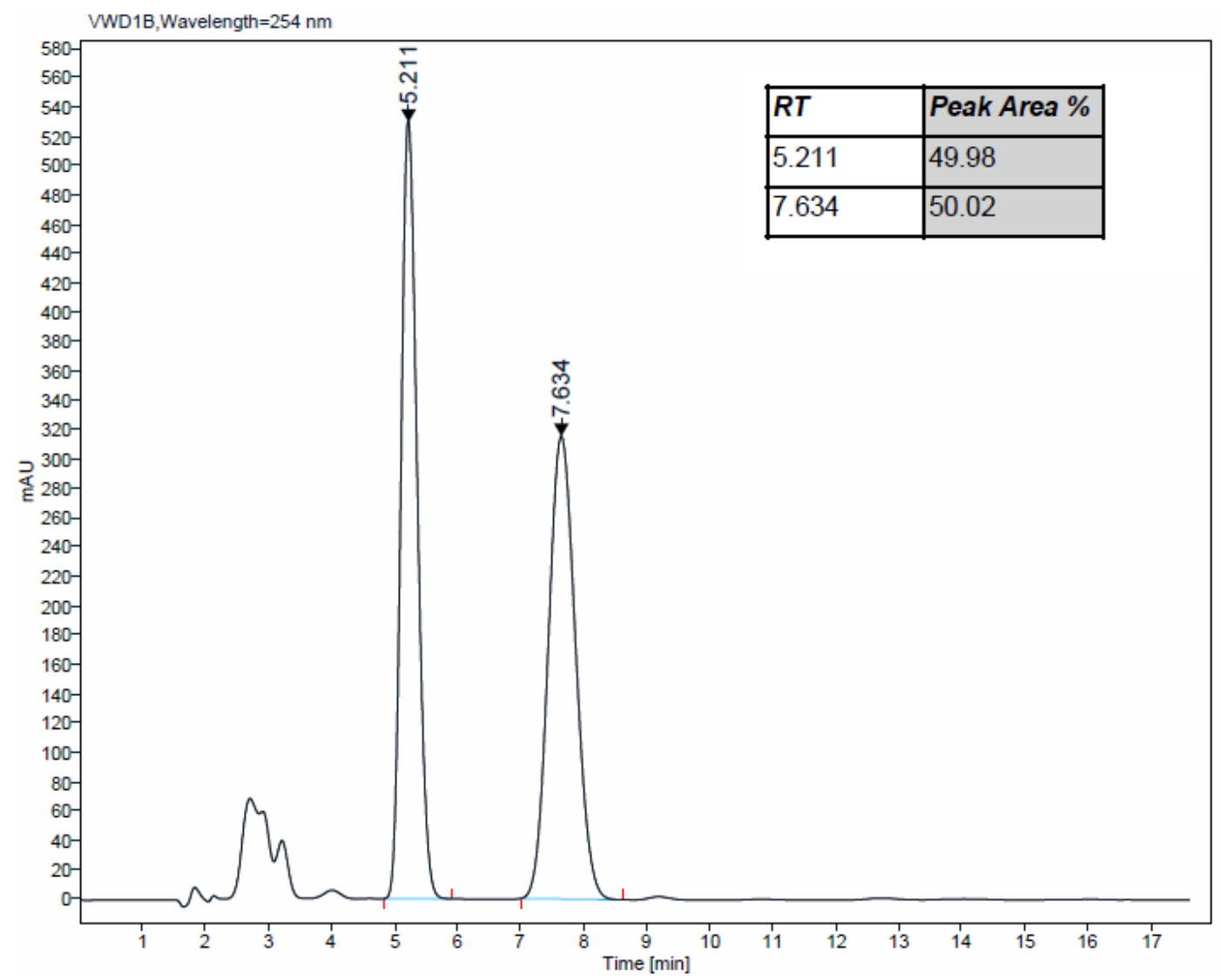




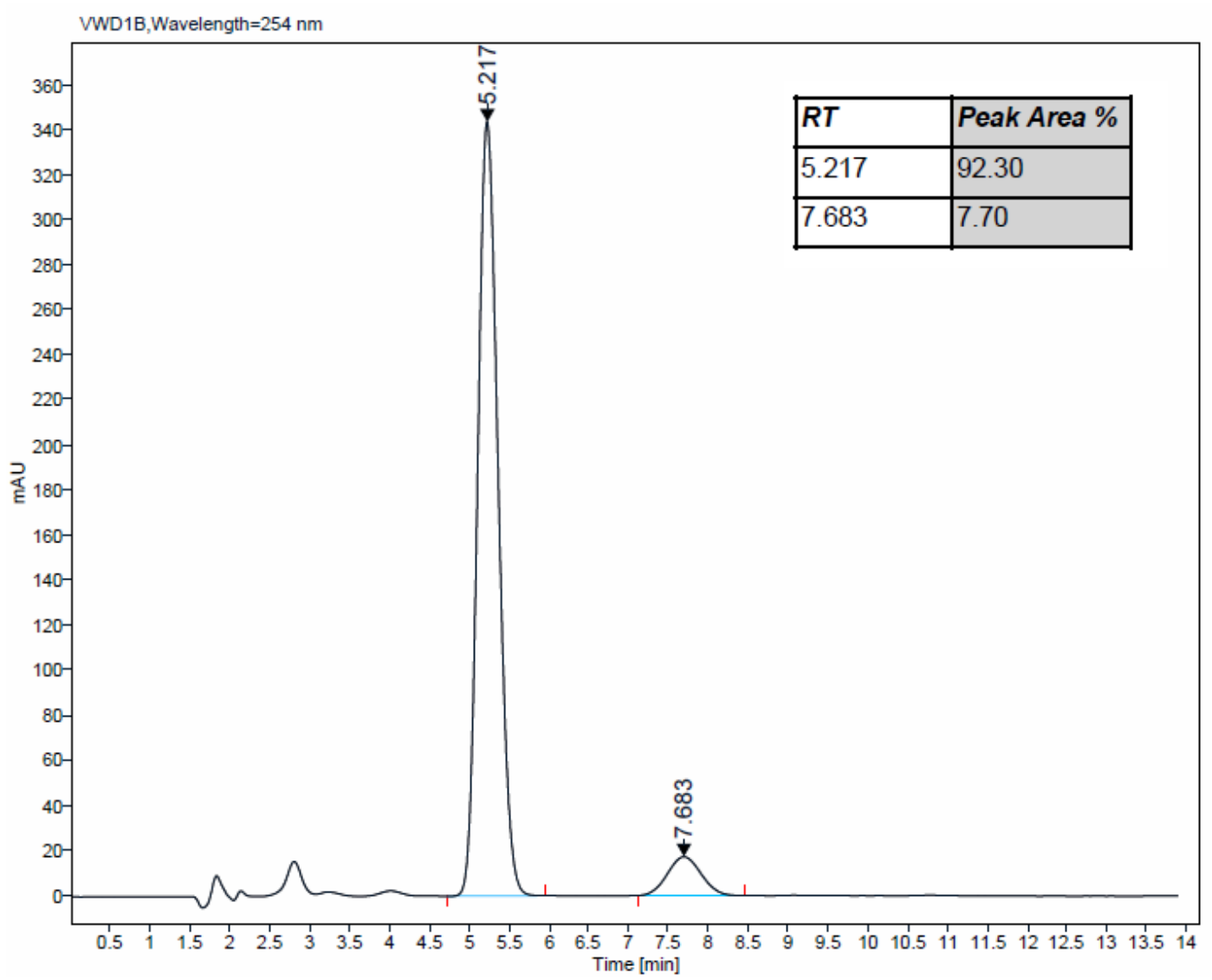

(E)-9b-hydroxy-7-methoxy-3a-methyl-5-(2-(trifluoromethyl)benzylidene)-1,2,3a,4,5,9bhexahydro-3H-cyclopenta[a]naphthalen-3-one (2h)<smiles>CCCC1(O)C(=O)CCc2ccc(OC)cc2C1=Cc1ccccc1C(F)(F)F</smiles>

Yellow oil (55 mg, 92\%) obtained from the general procedure (4) using 1a (44 mg, $0.15 \mathrm{mmol}$ ) and 3-methoxyphenylboronic acid (46 $\mathrm{mg}, 0.30 \mathrm{mmol}$ ).

$\mathbf{R}_{\mathbf{f}}=0.20$ (petroleum ether/EtOAc 80:20).

SFC (Chiralpak ID $s \mathrm{CO}_{2} / \mathrm{MeOH}$ 95:5, $2 \mathrm{~mL} / \mathrm{min}, \mathrm{P}=100$ bar): $\mathrm{Rt}=12.8 \mathrm{~min}$ (major), 14.2 $\min$ (minor).

$[\boldsymbol{\alpha}]_{\mathbf{D}}{ }^{\mathbf{2 0}}=+217\left(\mathrm{c}=2.2, \mathrm{CHCl}_{3}\right)$ for an enantiomeric excess of $88 \%$.

${ }^{1}$ H NMR $\left(400 \mathrm{MHz}, \mathrm{CDCl}_{3}, \delta\right): 7.72-7.67(\mathrm{~m}, 1 \mathrm{H}), 7.63(\mathrm{~d}, J=8.6 \mathrm{~Hz}, 1 \mathrm{H}), 7.56-7.46(\mathrm{~m}$, $1 \mathrm{H}), 7.43-7.37(\mathrm{~m}, 1 \mathrm{H}), 7.37-7.23(\mathrm{~m}, 2 \mathrm{H}), 7.15(\mathrm{~d}, J=2.6 \mathrm{~Hz}, 1 \mathrm{H}), 6.98(\mathrm{dd}, J=8.6,2.6$ $\mathrm{Hz}, 1 \mathrm{H}), 3.86(\mathrm{~s}, 3 \mathrm{H}), 2.73-2.48(\mathrm{~m}, 2 \mathrm{H}), 2.47-2.35(\mathrm{~m}, 3 \mathrm{H}), 2.34-2.21(\mathrm{~m}, 1 \mathrm{H}), 1.91(\mathrm{~d}, J$ $=1.7 \mathrm{~Hz}, 1 \mathrm{H}), 0.99(\mathrm{~s}, 3 \mathrm{H})$.

${ }^{13}$ C NMR (101 MHz, $\left.\mathrm{CDCl}_{3}, \delta\right): 220.2,159.4,135.8,134.9,134.4,133.1,131.7,131.1,129.0$ $(\mathrm{q}, J=29.6 \mathrm{~Hz}), 128.1,127.4,126.1(\mathrm{q}, J=6.2,5.6 \mathrm{~Hz}), 124.23,124.21(\mathrm{q}, J=274.0 \mathrm{~Hz})$, $115.6,108.8,79.9,55.4,54.3,36.0,35.4,34.8,14.1$.

${ }^{19}$ F NMR $\left(376 \mathrm{MHz}, \mathrm{CDCl}_{3}, \delta\right):-60.6$.

HRMS (ESI): Calculated for $\mathrm{C}_{23} \mathrm{H}_{21} \mathrm{~F}_{3} \mathrm{O}_{3} \mathrm{Na}(\mathrm{M}+\mathrm{Na})^{+} 425.1340$, found 425.1333 . 

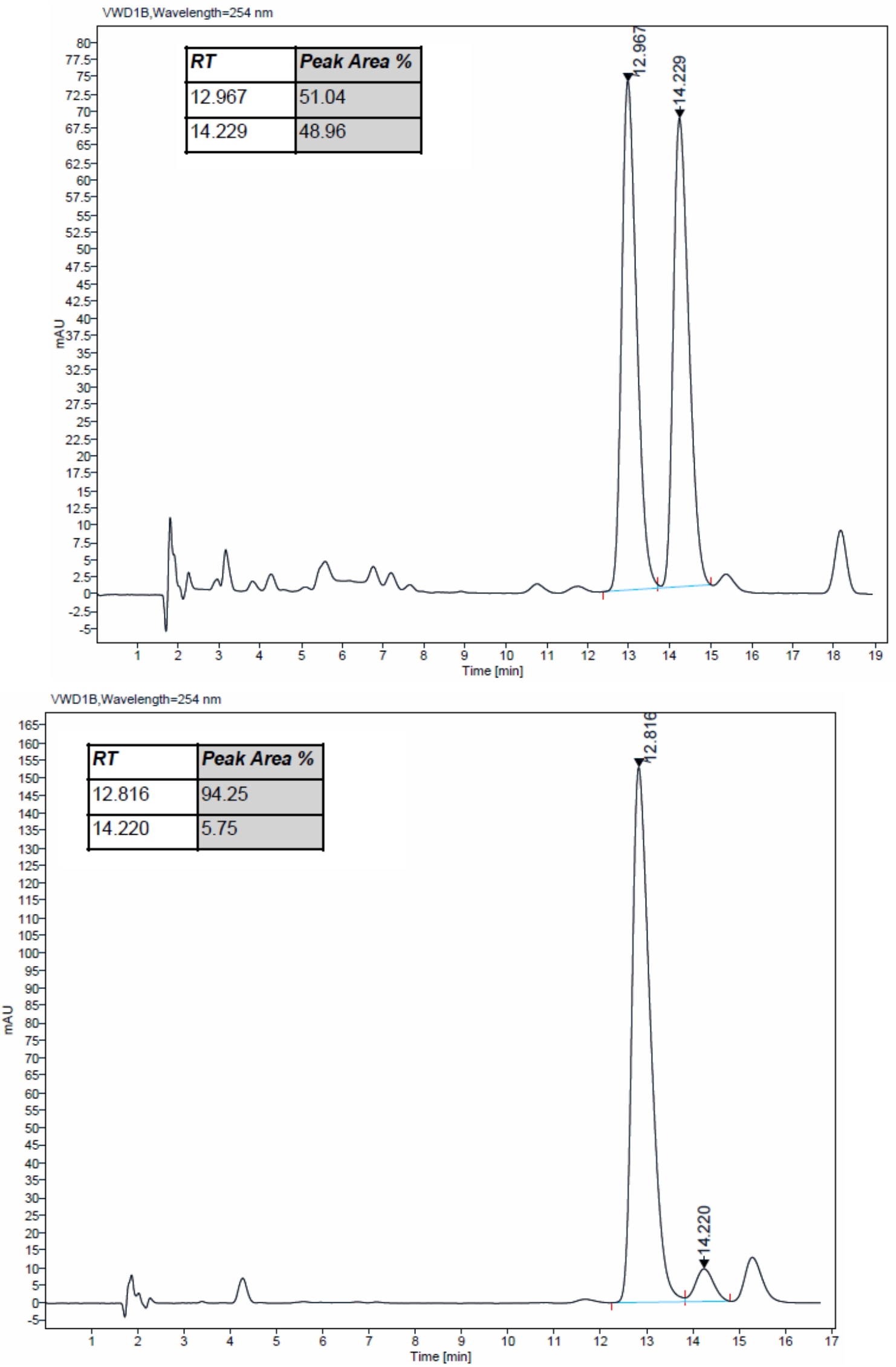

(E)-N-(9b-hydroxy-3a-methyl-3-oxo-5-(2-(trifluoromethyl)benzylidene)-2,3,3a,4,5,9bhexahydro-1H-cyclopenta[a]naphthalen-7-yl)acetamide (2i) 


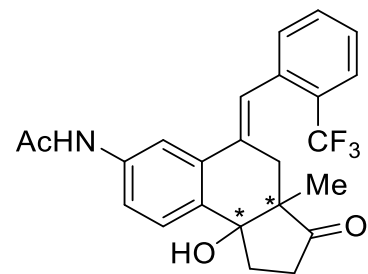

Orange oil (61 mg, 95\%) obtained from the general procedure (4) using 1a (44 mg, $0.15 \mathrm{mmol}$ ) and 3-acetamidophenylboronic acid (54 $\mathrm{mg}, 0.30 \mathrm{mmol})$.

$\mathbf{R}_{\mathbf{f}}=0.11$ (petroleum ether/EtOAc 50:50).

SFC (Chiralpak ADH $s \mathrm{CO}_{2} / \mathrm{MeOH} 90: 10,1 \mathrm{~mL} / \mathrm{min}, \mathrm{P}=100$ bar): $\mathrm{Rt}=27.8 \mathrm{~min}$ (minor), 30.7 $\min$ (major).

$[\boldsymbol{\alpha}]_{\mathbf{D}}{ }^{\mathbf{2 0}}=+288\left(\mathrm{c}=2.2, \mathrm{CHCl}_{3}\right)$ for an enantiomeric excess of $83 \%$.

${ }^{1} \mathbf{H}$ NMR $\left(400 \mathrm{MHz}, \mathrm{CDCl}_{3}, \delta\right): 7.78-7.65(\mathrm{~m}, 3 \mathrm{H}), 7.65-7.58(\mathrm{~m}, 1 \mathrm{H}), 7.52(\mathrm{t}, J=7.6 \mathrm{~Hz}$, $1 \mathrm{H}), 7.40(\mathrm{t}, J=7.6 \mathrm{~Hz}, 1 \mathrm{H}), 7.30(\mathrm{~s}, 1 \mathrm{H}), 7.27-7.22(\mathrm{~m}, 2 \mathrm{H}), 2.66-2.54(\mathrm{~m}, 1 \mathrm{H}), 2.54-$ $2.48(\mathrm{~m}, 1 \mathrm{H}), 2.48-2.35(\mathrm{~m}, 3 \mathrm{H}), 2.33-2.23(\mathrm{~m}, 1 \mathrm{H}), 2.21(\mathrm{~s}, 3 \mathrm{H}), 1.81(\mathrm{~d}, J=2.2 \mathrm{~Hz}, 1 \mathrm{H})$, $0.99(\mathrm{~s}, 3 \mathrm{H})$.

${ }^{13}$ C NMR (101 MHz, $\left.\mathrm{CDCl}_{3}, \delta\right): 220.1,168.8,137.8,136.6,135.7,134.3,134.0,131.7,131.1$, $128.9(\mathrm{q}, J=29.5 \mathrm{~Hz}), 127.6,127.4,126.0(\mathrm{q}, J=5.3 \mathrm{~Hz}), 124.5,124.2(\mathrm{q}, J=273.9 \mathrm{~Hz})$, $121.5,115.4,79.8,54.2,35.9,35.3,34.6,24.5,14.0$.

${ }^{19}$ F NMR $\left(376 \mathrm{MHz}, \mathrm{CDCl}_{3}, \delta\right):-60.5$.

HRMS (ESI): Calculated for $\mathrm{C}_{24} \mathrm{H}_{22} \mathrm{~F}_{3} \mathrm{O}_{3} \mathrm{NNa}(\mathrm{M}+\mathrm{Na})^{+} 452.1449$, found 452.1443 .

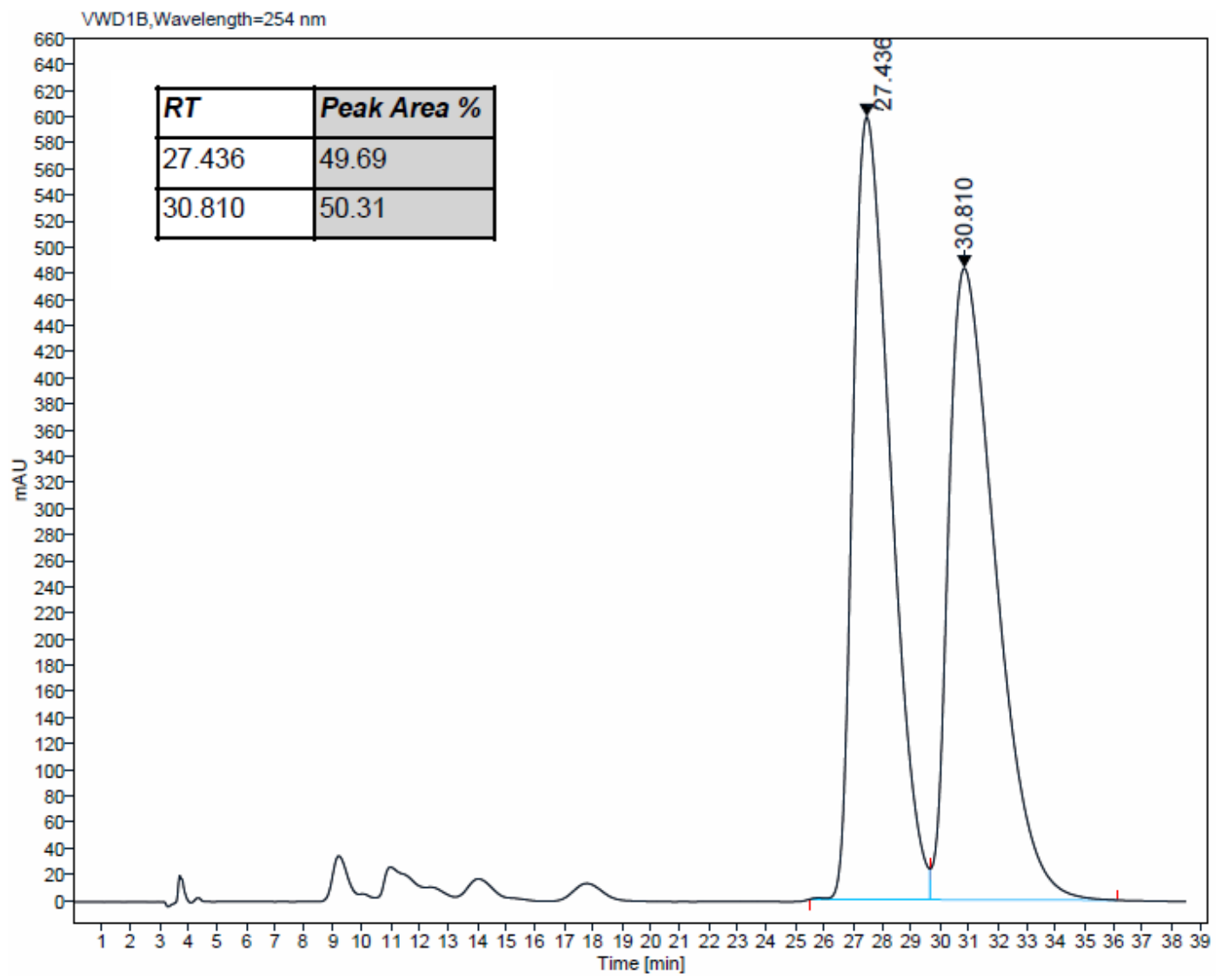




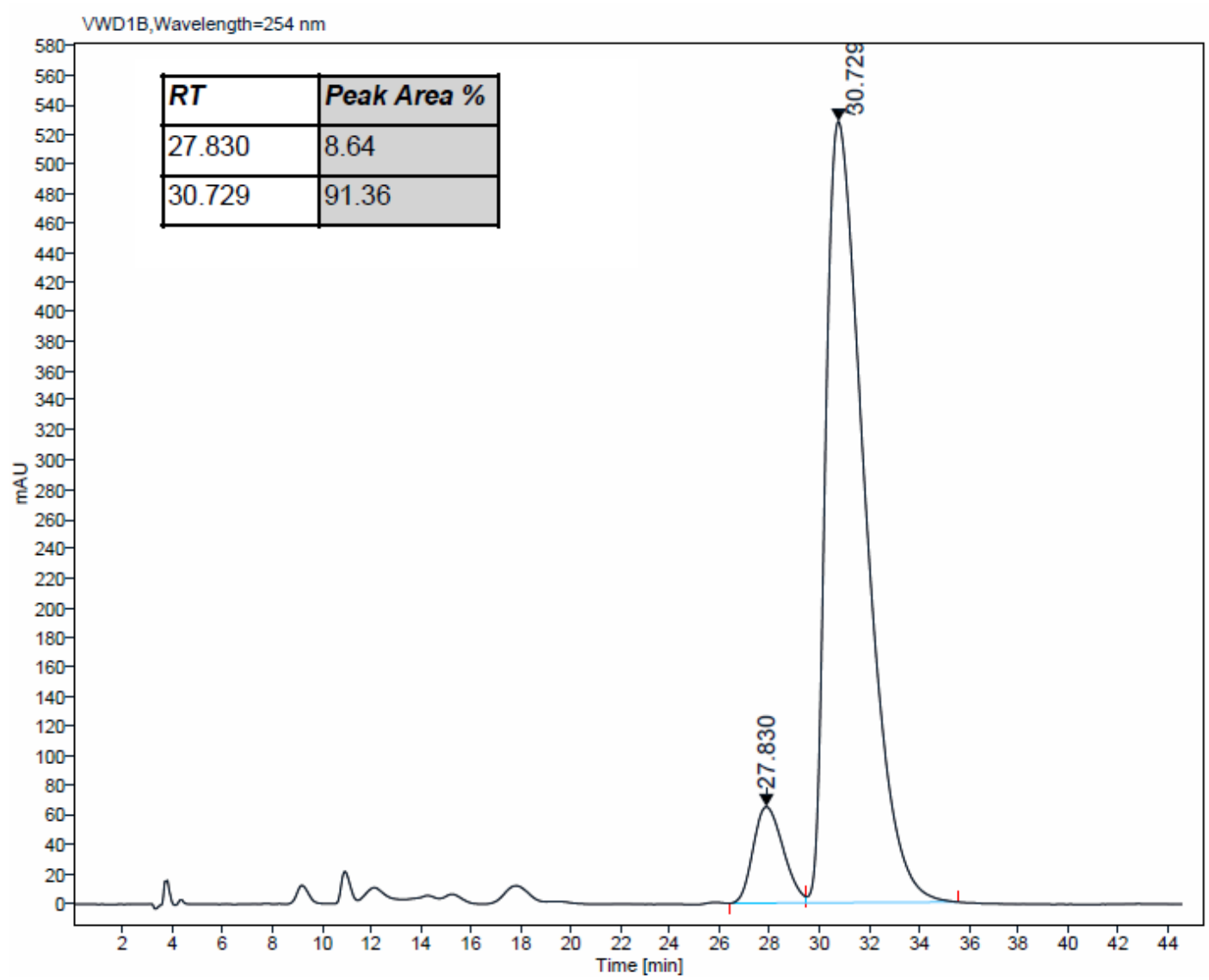

(E)-7-chloro-9b-hydroxy-3a-methyl-5-(2-(trifluoromethyl)benzylidene)-1,2,3a,4,5,9bhexahydro-3H-cyclopenta $[a]$ naphthalen-3-one $(2 \mathrm{j})$<smiles>CC1(C)CCC2(O)c3ccc(Cl)cc3/C(=C/c3ccccc3C(F)(F)F)CC12O</smiles>

Yellow oil (57 mg, 94\%) obtained from the general procedure (4) using 1a (44 mg, $0.15 \mathrm{mmol}$ ) and 3-chlorophenylboronic acid (47 $\mathrm{mg}, 0.30 \mathrm{mmol}$ ).

$\mathbf{R}_{\mathbf{f}}=0.18$ (petroleum ether/EtOAc 80:20).

SFC (Chiralpak ID $s \mathrm{CO}_{2} / \mathrm{MeOH} 90: 10,2 \mathrm{~mL} / \mathrm{min}, \mathrm{P}=100$ bar): $\mathrm{Rt}=4.9 \mathrm{~min}$ (major), $5.4 \mathrm{~min}$ (minor).

$[\alpha]_{\mathbf{D}}{ }^{\mathbf{2 0}}=+238\left(\mathrm{c}=2.0, \mathrm{CHCl}_{3}\right)$ for an enantiomeric excess of $82 \%$.

${ }^{1} \mathrm{H}$ NMR $\left(400 \mathrm{MHz}, \mathrm{CDCl}_{3}, \delta\right): 7.70(\mathrm{dd}, J=7.9,1.3 \mathrm{~Hz}, 1 \mathrm{H}), 7.65(\mathrm{~d}, J=8.5 \mathrm{~Hz}, 1 \mathrm{H}), 7.63$ $(\mathrm{d}, J=2.2 \mathrm{~Hz}, 1 \mathrm{H}), 7.56-7.50(\mathrm{~m}, 1 \mathrm{H}), 7.41(\mathrm{t}, J=7.9 \mathrm{~Hz}, 1 \mathrm{H}), 7.36(\mathrm{dd}, J=8.5,2.1 \mathrm{~Hz}$, $1 \mathrm{H}), 7.31(\mathrm{~s}, 1 \mathrm{H}), 7.23(\mathrm{~d}, J=7.9 \mathrm{~Hz}, 1 \mathrm{H}), 2.73-2.53(\mathrm{~m}, 1 \mathrm{H}), 2.49(\mathrm{dd}, J=14.2,2.1 \mathrm{~Hz}$, $1 \mathrm{H}), 2.47-2.33(\mathrm{~m}, 3 \mathrm{H}), 2.32-2.20(\mathrm{~m}, 1 \mathrm{H}), 1.99(\mathrm{~d}, J=1.7 \mathrm{~Hz}, 1 \mathrm{H}), 0.97(\mathrm{~s}, 3 \mathrm{H})$.

${ }^{13}$ C NMR (101 MHz, $\left.\mathrm{CDCl}_{3}, \delta\right): 219.5,138.9,135.4,135.3,134.3,133.3,131.8,131.0,129.3$, $129.0(\mathrm{q}, J=29.9 \mathrm{~Hz}), 128.4,127.7,126.1$ (q, $J=5.4 \mathrm{~Hz}), 125.3,124.23,124.21$ (q, $J=273.8$ Hz), 79.8, 54.1, 35.9, 35.2, 34.6, 13.8 .

${ }^{19}$ F NMR $\left(376 \mathrm{MHz}, \mathrm{CDCl}_{3}, \delta\right):-60.5$.

HRMS (ESI): Calculated for $\mathrm{C}_{22} \mathrm{H}_{18} \mathrm{~F}_{3} \mathrm{O}_{2} \mathrm{ClNa}(\mathrm{M}+\mathrm{Na})^{+} 429.0845$, found 429.0839. 

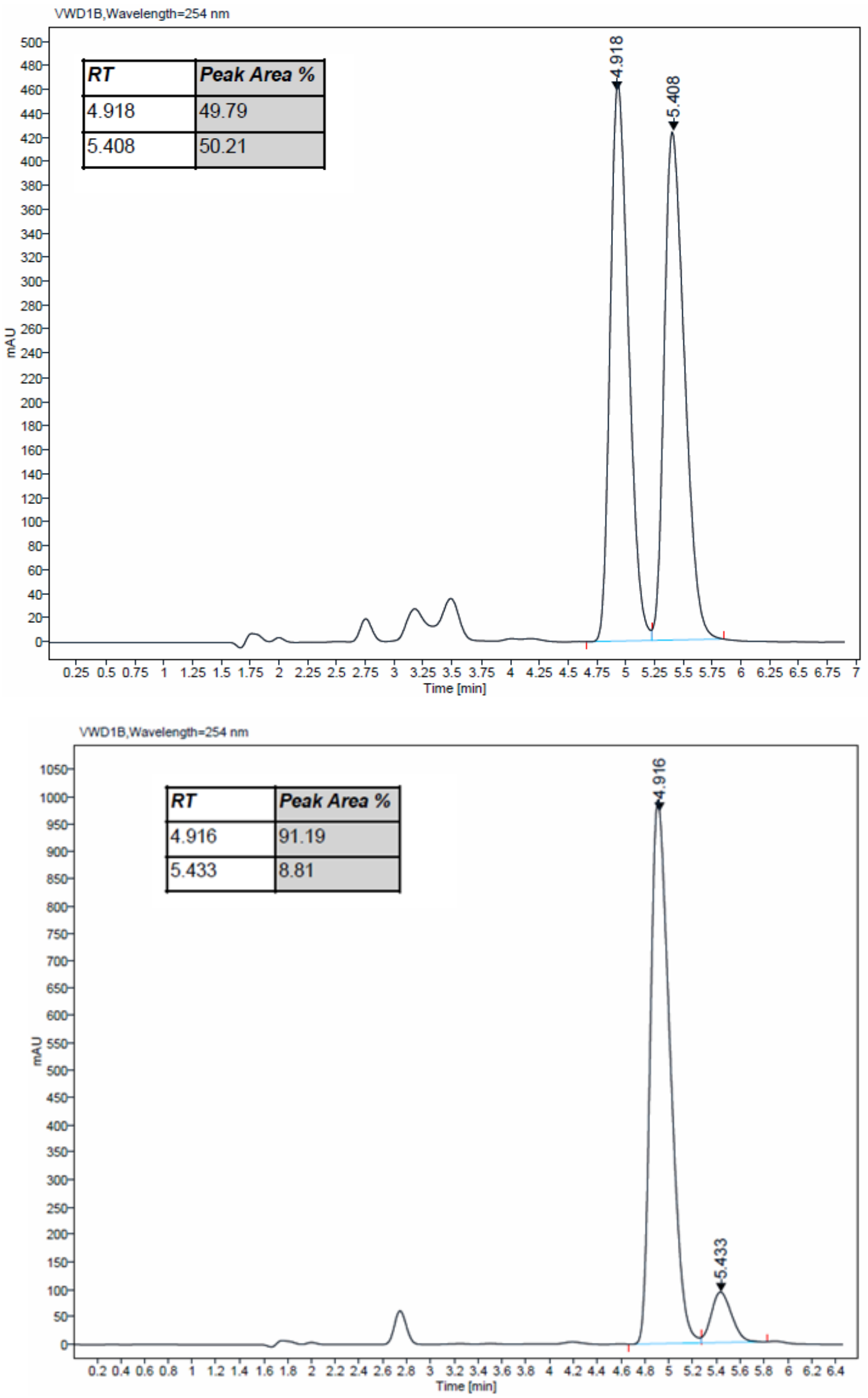

(E)-4a-hydroxy-6-methoxy-10a-methyl-9-(2-(trifluoromethyl)benzylidene)$3,4,4 a, 9,10,10 a-h e x a h y d r o p h e n a n t h r e n-1(2 H)$-one $(2 \mathrm{k})$ 
<smiles>COc1ccc(/C(=C/c2ccccc2C(F)(F)F)C2(O)CCCCC2(O)C(=O)O)cc1</smiles>

Orange oil (52 mg, 84\%) obtained from the general procedure (4) using $1 \mathbf{b}$ (46 mg, $0.15 \mathrm{mmol}$ ) and 4-methoxyphenylboronic acid (46 $\mathrm{mg}, 0.30 \mathrm{mmol})$.

$\mathbf{R}_{\mathbf{f}}=0.16$ (petroleum ether/EtOAc 80:20).

SFC (Chiralpak ASH $s \mathrm{CO}_{2} / \mathrm{MeOH} 80: 20,2 \mathrm{~mL} / \mathrm{min}, \mathrm{P}=100$ bar): $\mathrm{Rt}=3.4 \mathrm{~min}$ (minor), 5.4 $\min$ (major).

$[\boldsymbol{\alpha}]_{\mathbf{D}}{ }^{\mathbf{2 0}}=+105\left(\mathrm{c}=0.7, \mathrm{CHCl}_{3}\right)$ for an enantiomeric excess of $87 \%$.

${ }^{1} \mathbf{H}$ NMR $\left(400 \mathrm{MHz}, \mathrm{CDCl}_{3}, \delta\right): 7.71-7.65(\mathrm{~m}, 2 \mathrm{H}), 7.50(\mathrm{t}, J=7.7 \mathrm{~Hz}, 1 \mathrm{H}), 7.37(\mathrm{t}, J=7.7$ $\mathrm{Hz}, 1 \mathrm{H}), 7.26(\mathrm{~d}, J=7.7 \mathrm{~Hz}, 1 \mathrm{H}), 7.24-7.18(\mathrm{~m}, 1 \mathrm{H}), 7.16(\mathrm{~d}, J=2.8 \mathrm{~Hz}, 1 \mathrm{H}), 6.89(\mathrm{dd}, J=$ $8.8,2.8 \mathrm{~Hz}, 1 \mathrm{H}), 3.86(\mathrm{~s}, 3 \mathrm{H}), 3.00(\mathrm{dd}, J=14.9,2.5 \mathrm{~Hz}, 1 \mathrm{H}), 2.68-2.55(\mathrm{~m}, 1 \mathrm{H}), 2.42$ (dd, $J$ $=14.9,0.8 \mathrm{~Hz}, 1 \mathrm{H}), 2.39-2.33(\mathrm{~m}, 1 \mathrm{H}), 2.32-2.21(\mathrm{~m}, 1 \mathrm{H}), 2.21-2.15(\mathrm{~m}, 1 \mathrm{H}), 2.04-1.97$ (m, 1H), $1.96(\mathrm{~d}, J=2.2 \mathrm{~Hz}, 1 \mathrm{H}), 1.94-1.85(\mathrm{~m}, 1 \mathrm{H}), 1.01(\mathrm{~s}, 3 \mathrm{H})$.

${ }^{13}$ C NMR (101 MHz, $\left.\mathrm{CDCl}_{3}, \delta\right): 213.7,160.4,144.1,136.4,133.6,131.6,131.1,129.0$ (q, $J=$ $29.7 \mathrm{~Hz}), 127.1,126.0(\mathrm{q}, J=5.8 \mathrm{~Hz}), 125.8,125.5,124.2(\mathrm{q}, J=273.8 \mathrm{~Hz}), 121.3,114.5$, $109.9,78.6,55.4,53.5,37.1,37.0,36.9,21.0,15.4$.

${ }^{19}$ F NMR $\left(376 \mathrm{MHz}, \mathrm{CDCl}_{3}, \delta\right):-60.9$.

HRMS (ESI): Calculated for $\mathrm{C}_{24} \mathrm{H}_{23} \mathrm{~F}_{3} \mathrm{O}_{4} \mathrm{Na}(\mathrm{M}+\mathrm{Na})^{+} 439.1497$, found 439.1490 .

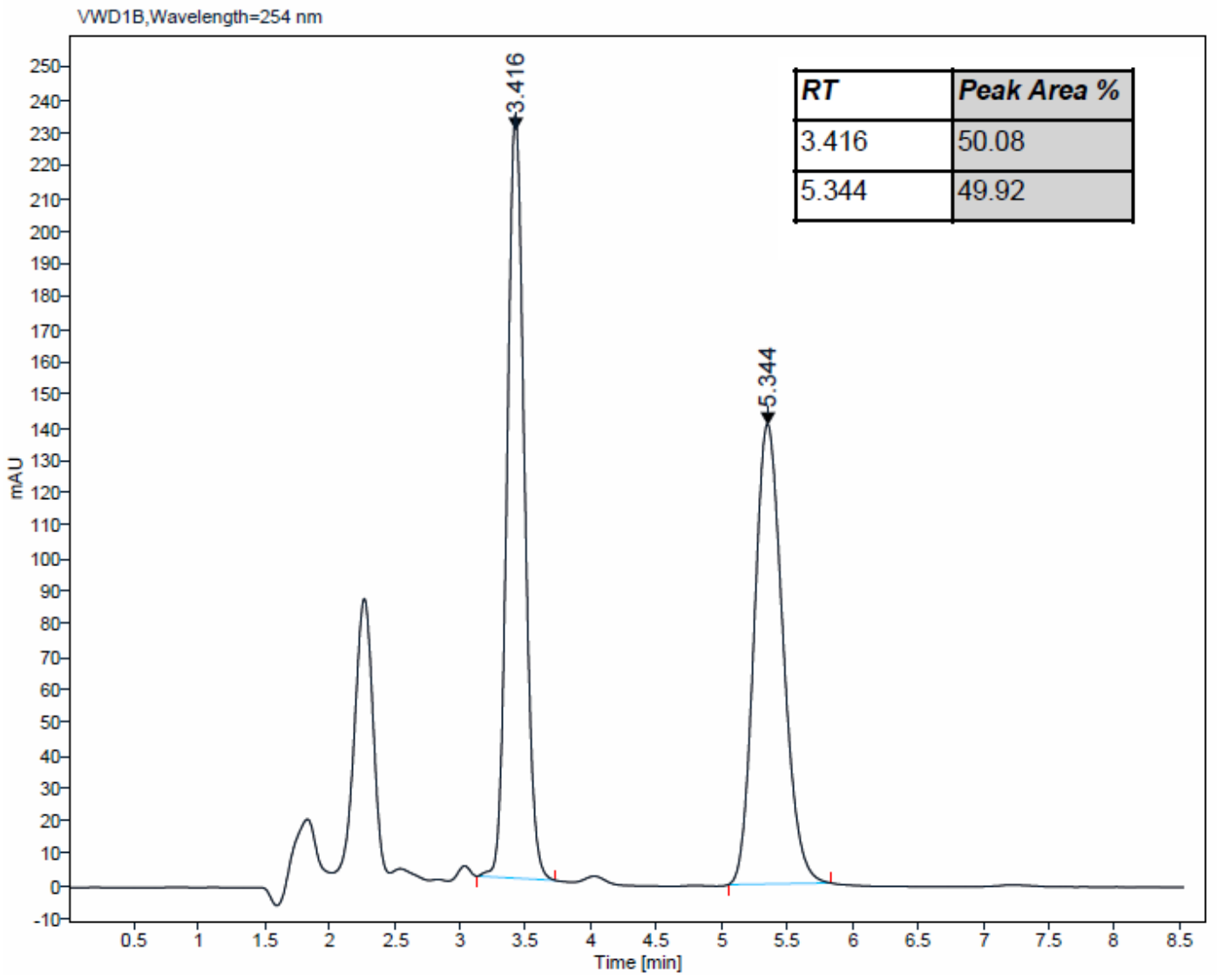




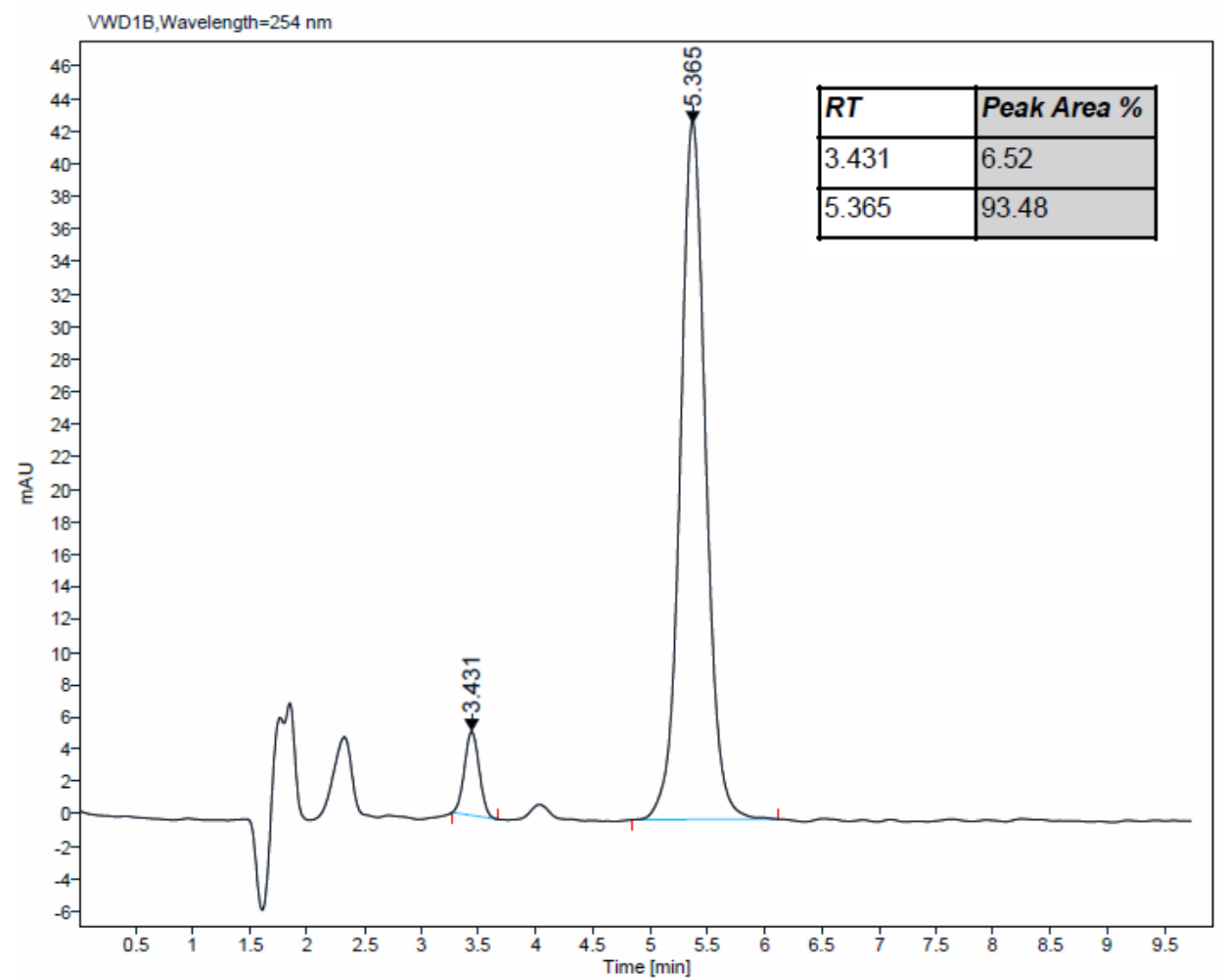

(E)-6-(tert-butyl)-4a-hydroxy-10a-methyl-9-(2-(trifluoromethyl)benzylidene)$3,4,4 a, 9,10,10 a-h e x a h y d r o p h e n a n t h r e n-1(2 H)$-one (2I)<smiles>CC(C)(C)c1ccc2c(c1)C1(O)CCCC(=O)C1(O)CC2=Cc1ccccc1F</smiles>

Orange oil (61 mg, 92\%) obtained from the general procedure (4) using $\mathbf{1 b}$ (46 mg, $0.15 \mathrm{mmol}$ ) and 4-tert-butylphenylboronic acid (53 $\mathrm{mg}, 0.30 \mathrm{mmol})$.

Scale-up reaction: Orange oil (483 mg, 91\%) obtained from the general procedure (4) using $\mathbf{1 b}$ (368 mg, $1.2 \mathrm{mmol}$ ) and 4-tert-butylphenylboronic acid (424 mg, $2.4 \mathrm{mmol}$ ).

$\mathbf{R}_{\mathbf{f}}=0.36$ (petroleum ether/EtOAc 80:20).

SFC (Chiralpak ASH $s \mathrm{CO}_{2} / \mathrm{MeOH} 90: 10,2 \mathrm{~mL} / \mathrm{min}, \mathrm{P}=100 \mathrm{bar}$ ): $\mathrm{Rt}=4.3 \mathrm{~min}$ (minor), 6.1 $\min$ (major).

$[\alpha]_{\mathbf{D}} \mathbf{D}^{\mathbf{2 0}}=+108\left(\mathrm{c}=1.3, \mathrm{CHCl}_{3}\right)$ for an enantiomeric excess of $87 \%$.

${ }^{1}$ H NMR (400 MHz, $\left.\mathrm{CDCl}_{3}, \delta\right): 7.74-7.63(\mathrm{~m}, 3 \mathrm{H}), 7.50(\mathrm{t}, J=7.6 \mathrm{~Hz}, 1 \mathrm{H}), 7.43-7.34(\mathrm{~m}$, 2H), $7.33(\mathrm{~s}, 1 \mathrm{H}), 7.29-7.22(\mathrm{~m}, 1 \mathrm{H}), 3.00(\mathrm{dd}, J=14.7,2.1 \mathrm{~Hz}, 1 \mathrm{H}), 2.66-2.53(\mathrm{~m}, 1 \mathrm{H})$, $2.42(\mathrm{dd}, J=14.7,2.1 \mathrm{~Hz}, 1 \mathrm{H}), 2.39-2.15(\mathrm{~m}, 3 \mathrm{H}), 2.04-1.97(\mathrm{~m}, 1 \mathrm{H}), 1.94(\mathrm{~d}, J=2.1 \mathrm{~Hz}$, $1 \mathrm{H}), 1.93-1.83(\mathrm{~m}, 1 \mathrm{H}), 1.35(\mathrm{~s}, 9 \mathrm{H}), 1.03(\mathrm{~s}, 3 \mathrm{H})$.

${ }^{13}$ C NMR (101 MHz, $\left.\mathrm{CDCl}_{3}, \delta\right): 213.9,152.2,142.1,136.3,134.0,131.6,131.1,130.2,129.0$ $(\mathrm{q}, J=29.5 \mathrm{~Hz}), 127.2,126.0(\mathrm{q}, J=5.6 \mathrm{~Hz}), 125.1,124.2(\mathrm{q}, J=273.8 \mathrm{~Hz}), 123.6,122.4$, $122.3,78.7,53.6,37.4,37.1,36.9,34.9,31.3(3 \mathrm{C}), 21.0,15.5$.

${ }^{19}$ F NMR $\left(376 \mathrm{MHz}, \mathrm{CDCl}_{3}, \delta\right):-60.8$.

HRMS (ESI): Calculated for $\mathrm{C}_{27} \mathrm{H}_{29} \mathrm{~F}_{3} \mathrm{O}_{2} \mathrm{Na}(\mathrm{M}+\mathrm{Na})^{+} 465.2017$, found 465.2010. 

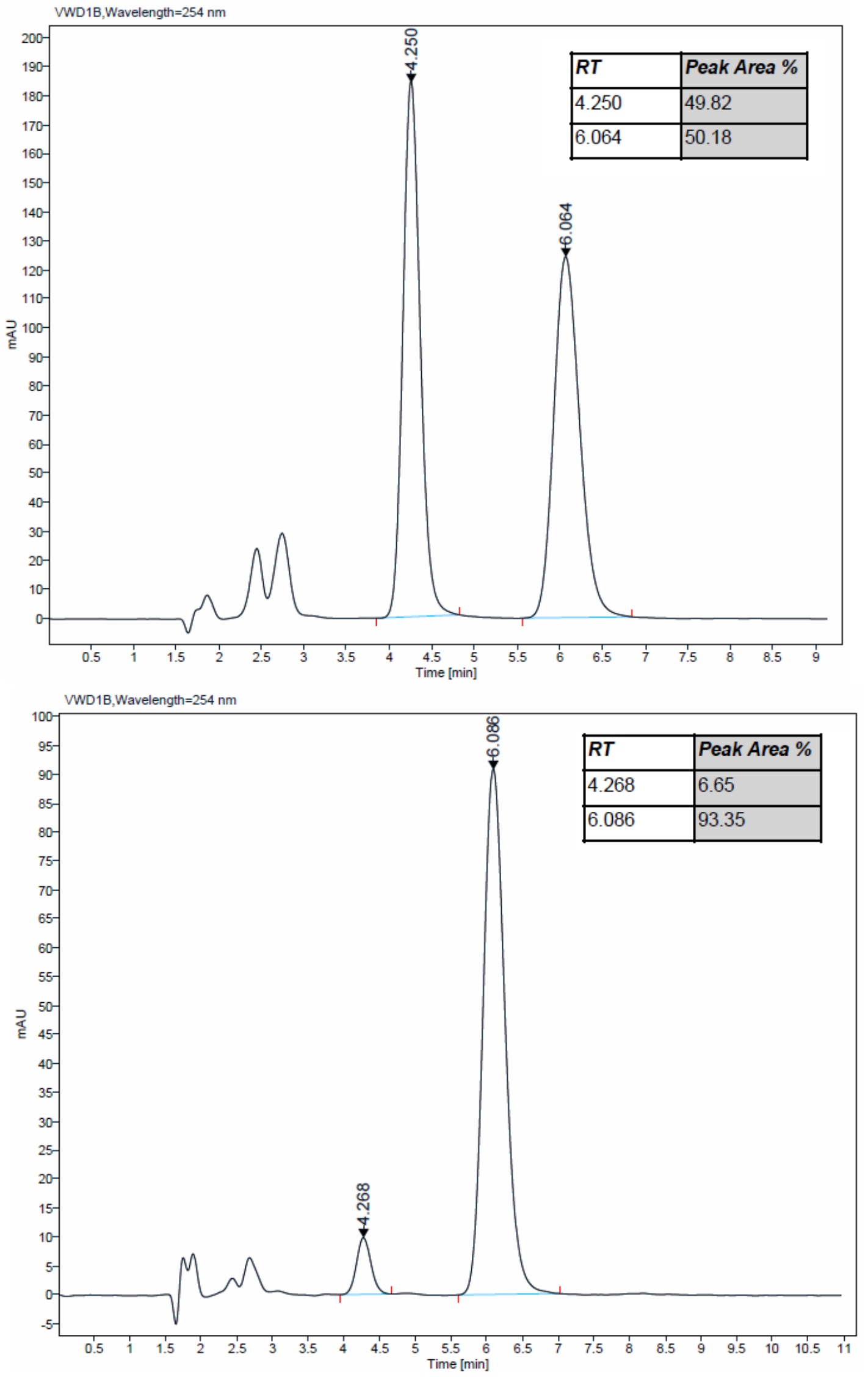

(E)-6-bromo-4a-hydroxy-10a-methyl-9-(2-(trifluoromethyl)benzylidene)-3,4,4a,9,10,10ahexahydrophenanthren-1(2H)-one (2m) 


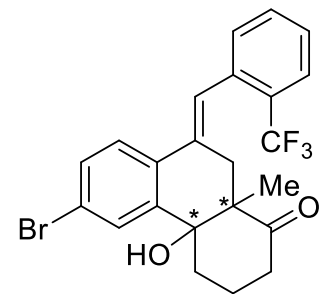

Orange oil (59 mg, 84\%) obtained from the general procedure (4) using $1 \mathbf{b}$ (46 mg, $0.15 \mathrm{mmol}$ ) and 4-bromophenylboronic acid (60 $\mathrm{mg}, 0.30 \mathrm{mmol})$.

$\mathbf{R}_{\mathbf{f}}=0.26$ (petroleum ether/EtOAc 80:20).

SFC (Chiralpak ASH $s \mathrm{CO}_{2} / \mathrm{MeOH} 80: 20,2 \mathrm{~mL} / \mathrm{min}, \mathrm{P}=100$ bar): $\mathrm{Rt}=3.9 \mathrm{~min}$ (minor), 6.7 $\min$ (major).

$[\boldsymbol{\alpha}]_{\mathbf{D}}{ }^{\mathbf{2 0}}=+76\left(\mathrm{c}=1.8, \mathrm{CHCl}_{3}\right)$ for an enantiomeric excess of $81 \%$.

${ }^{1} \mathbf{H}$ NMR $\left(400 \mathrm{MHz}, \mathrm{CDCl}_{3}, \delta\right): 7.80(\mathrm{~d}, J=2.2 \mathrm{~Hz}, 1 \mathrm{H}), 7.70(\mathrm{dd}, J=7.9,1.3 \mathrm{~Hz}, 1 \mathrm{H}), 7.60$ $(\mathrm{d}, J=8.6 \mathrm{~Hz}, 1 \mathrm{H}), 7.57-7.49(\mathrm{~m}, 1 \mathrm{H}), 7.44(\mathrm{dd}, J=8.6,2.2 \mathrm{~Hz}, 1 \mathrm{H}), 7.40(\mathrm{t}, J=7.9 \mathrm{~Hz}$, $1 \mathrm{H}), 7.33(\mathrm{t}, J=2.2 \mathrm{~Hz}, 1 \mathrm{H}), 7.26(\mathrm{~d}, J=7.9 \mathrm{~Hz}, 1 \mathrm{H}), 3.00(\mathrm{dd}, J=14.9,2.4 \mathrm{~Hz}, 1 \mathrm{H}), 2.68-$ $2.50(\mathrm{~m}, 1 \mathrm{H}), 2.42(\mathrm{dd}, J=14.9,0.8 \mathrm{~Hz}, 1 \mathrm{H}), 2.39-2.32(\mathrm{~m}, 1 \mathrm{H}), 2.28-2.13(\mathrm{~m}, 2 \mathrm{H}), 2.05$ $(\mathrm{d}, J=2.0 \mathrm{~Hz}, 1 \mathrm{H}), 1.99-1.92(\mathrm{~m}, 1 \mathrm{H}), 1.93-1.83(\mathrm{~m}, 1 \mathrm{H}), 0.99(\mathrm{~s}, 3 \mathrm{H})$.

${ }^{13}$ C NMR (101 MHz, $\left.\mathrm{CDCl}_{3}, \delta\right): 213.1,144.4,135.7,133.2,132.0,131.7,131.0,130.8,129.0$ $(\mathrm{q}, J=29.7 \mathrm{~Hz}), 128.9,127.5,126.2(\mathrm{q}, J=5.6 \mathrm{~Hz}), 125.7,124.1(\mathrm{q}, J=274.0 \mathrm{~Hz}), 124.0$, 123.1, 78.2, 53.1, 37.1, 36.9, 36.6, 20.8, 15.4.

${ }^{19}$ F NMR $\left(376 \mathrm{MHz}, \mathrm{CDCl}_{3}, \delta\right):-60.8$.

HRMS (ESI): Calculated for $\mathrm{C}_{23} \mathrm{H}_{20} \mathrm{~F}_{3} \mathrm{O}_{2} \mathrm{BrNa}(\mathrm{M}+\mathrm{Na})^{+}$487.0496, found 487.0490.

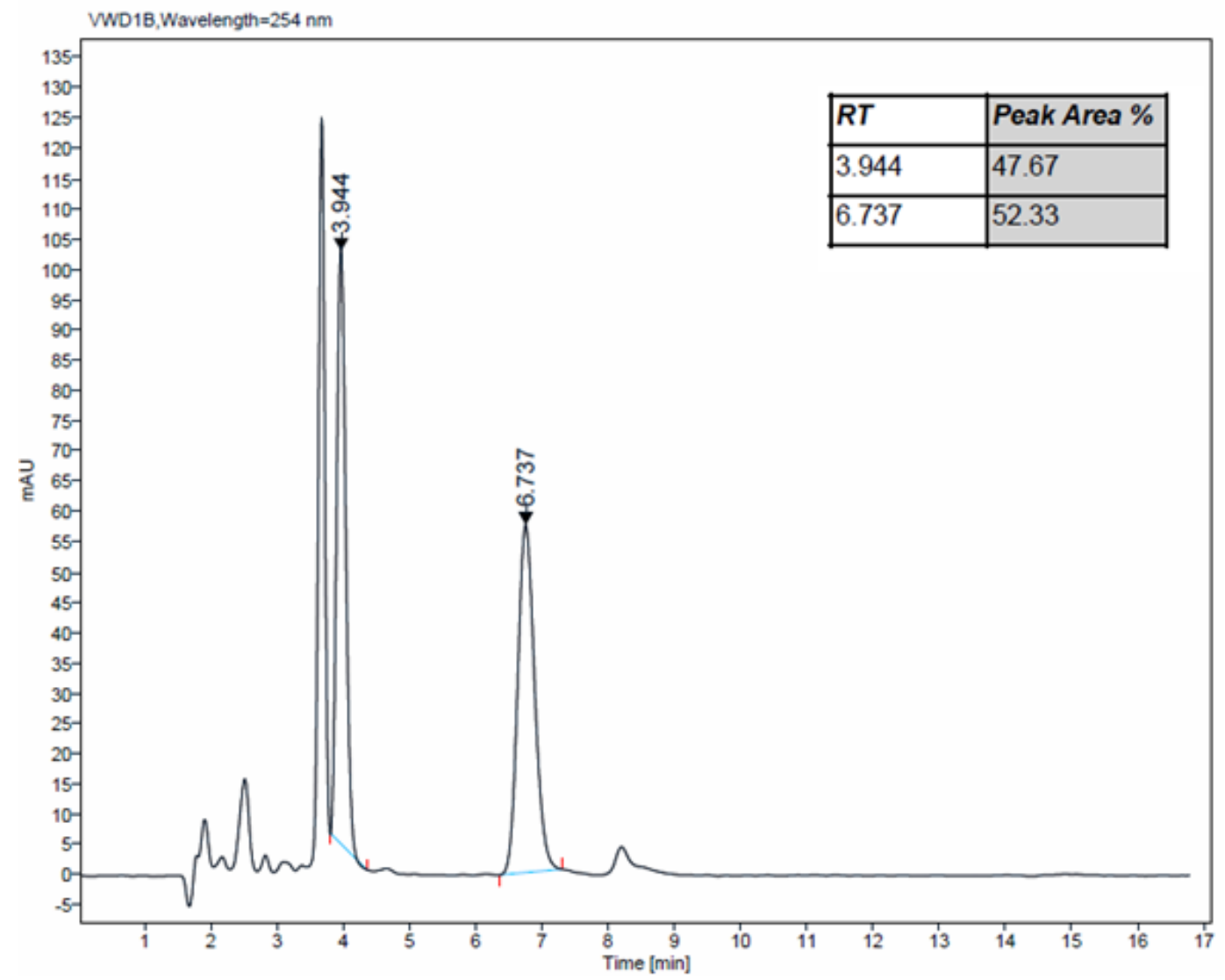




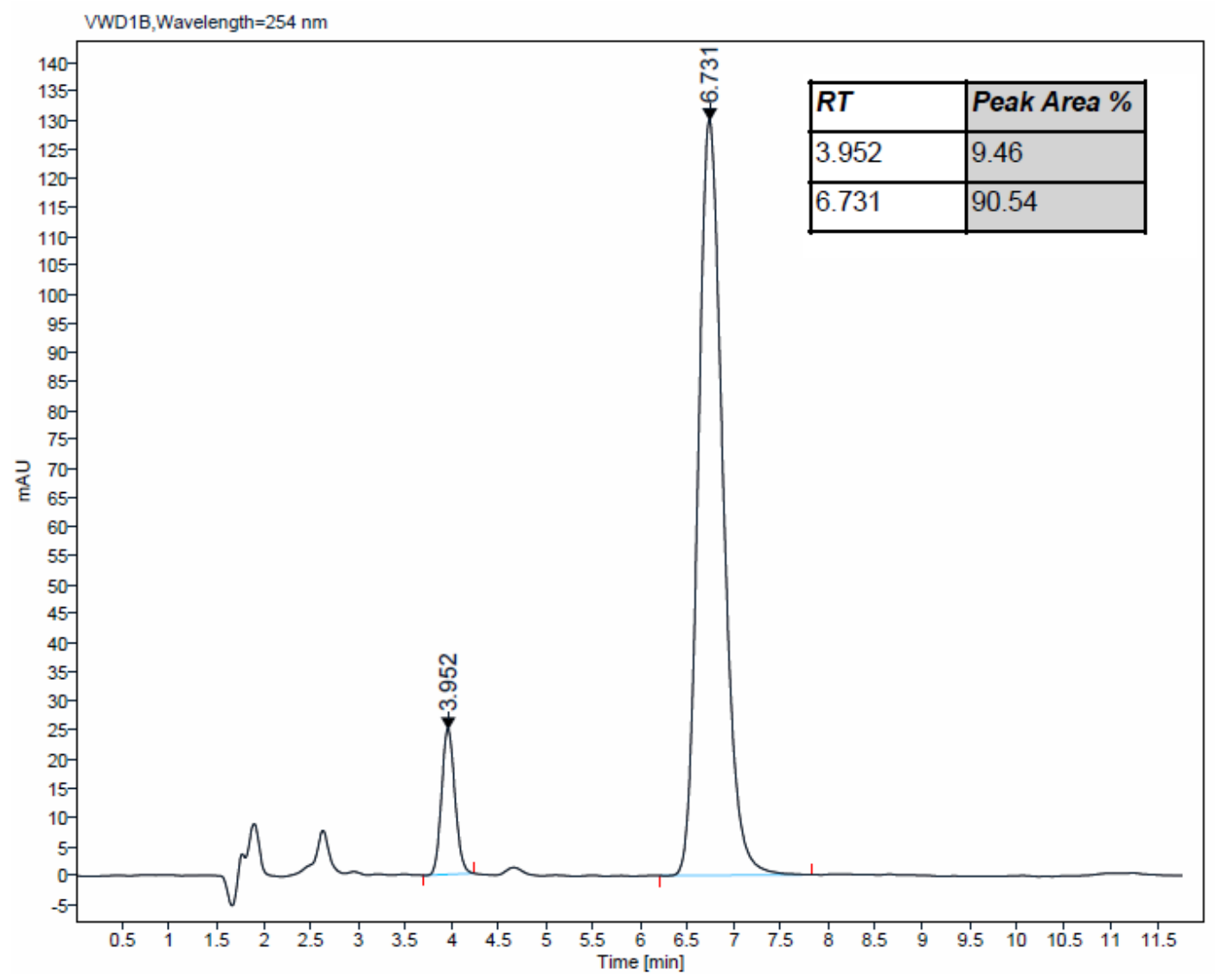

(E)-4a-hydroxy-7,10a-dimethyl-9-(2-(trifluoromethyl)benzylidene)-3,4,4a,9,10,10ahexahydrophenanthren-1(2H)-one (2n)<smiles>Cc1ccc2c(c1)C(=Cc1ccccc1F)CC1(O)C(=O)CCCC1(O)C2=O</smiles>

Orange oil (55 mg, 91\%) obtained from the general procedure (4) using $\mathbf{1 b}$ (46 mg, $0.15 \mathrm{mmol}$ ) and 3-tolylboronic acid (41 $\mathrm{mg}, 0.30 \mathrm{mmol})$.

$\mathbf{R}_{\mathbf{f}}=0.24$ (petroleum ether/EtOAc 80:20).

SFC (Chiralpak ASH $s \mathrm{CO}_{2} / \mathrm{MeOH} 80: 20,2 \mathrm{~mL} / \mathrm{min}, \mathrm{P}=100 \mathrm{bar}$ ): $\mathrm{Rt}=3.1 \mathrm{~min}$ (minor), 4.3 $\min$ (major).

$[\alpha]_{\mathbf{D}^{20}}=+91\left(\mathrm{c}=0.4, \mathrm{CHCl}_{3}\right)$ for an enantiomeric excess of $87 \%$.

${ }^{1} \mathbf{H}$ NMR $\left(400 \mathrm{MHz}, \mathrm{CDCl}_{3}, \delta\right): 7.70(\mathrm{~d}, J=7.8 \mathrm{~Hz}, 1 \mathrm{H}), 7.58-7.46(\mathrm{~m}, 3 \mathrm{H}), 7.39$ (t, $J=7.8$ $\mathrm{Hz}, 1 \mathrm{H}), 7.34(\mathrm{~s}, 1 \mathrm{H}), 7.30-7.24(\mathrm{~m}, 1 \mathrm{H}), 7.24-7.17(\mathrm{~m}, 1 \mathrm{H}), 3.12-2.94(\mathrm{~m}, 1 \mathrm{H}), 2.64-$ $2.51(\mathrm{~m}, 1 \mathrm{H}), 2.40(\mathrm{~s}, 3 \mathrm{H}), 2.46-2.33(\mathrm{~m}, 2 \mathrm{H}), 2.30-2.08(\mathrm{~m}, 2 \mathrm{H}), 2.04-1.94(\mathrm{~m}, 1 \mathrm{H}), 1.90$ $(\mathrm{d}, J=2.0 \mathrm{~Hz}, 1 \mathrm{H}), 1.88-1.82(\mathrm{~m}, 1 \mathrm{H}), 1.01(\mathrm{~s}, 3 \mathrm{H})$.

${ }^{13} \mathrm{C}$ NMR (101 MHz, $\left.\mathrm{CDCl}_{3}, \delta\right): 213.8,139.7,137.5,136.3,134.2,132.9,131.6,131.0,130.0$, $129.0(\mathrm{q}, J=29.7 \mathrm{~Hz}), 127.3,126.1(\mathrm{q}, J=4.9 \mathrm{~Hz}), 125.7,124.3,124.2(\mathrm{q}, J=273.8 \mathrm{~Hz})$, 123.1, 78.3, 53.6, 37.2, 37.1, 36.8, 21.3, 20.9, 15.6.

${ }^{19}$ F NMR $\left(376 \mathrm{MHz}, \mathrm{CDCl}_{3}, \delta\right):-60.8$.

HRMS (ESI): Calculated for $\mathrm{C}_{24} \mathrm{H}_{23} \mathrm{~F}_{3} \mathrm{O}_{2} \mathrm{Na}(\mathrm{M}+\mathrm{Na})^{+} 423.1548$, found 423.1543 . 

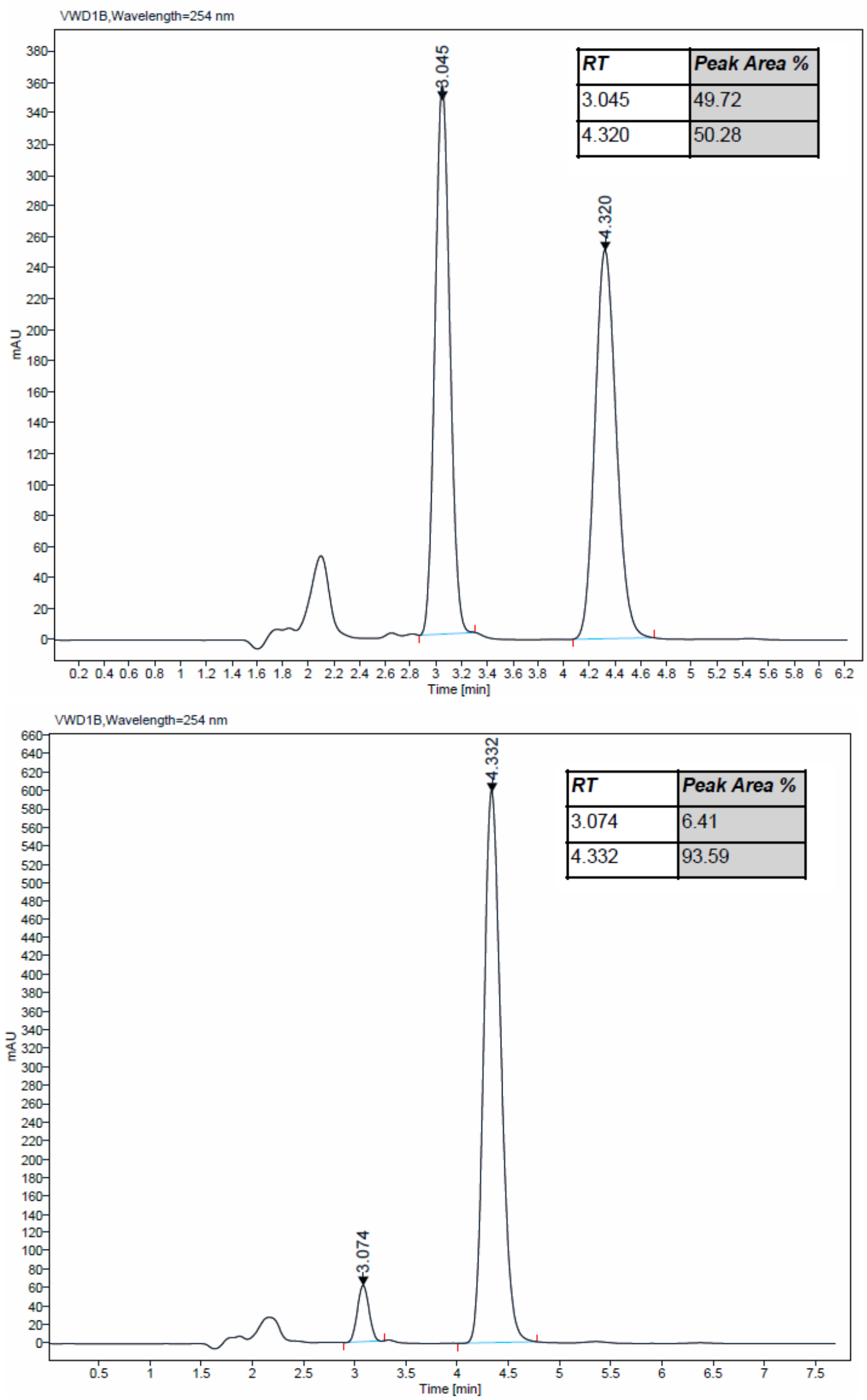

(E)- $N$-(4b-hydroxy-8a-methyl-8-oxo-10-(2-(trifluoromethyl)benzylidene)4b,5,6,7,8,8a,9,10-octahydrophenanthren-2-yl)acetamide (2o) 
<smiles>CC(=O)Nc1ccc2c(c1)C(=Cc1ccccc1F)CC1(O)C(=O)CCCC1(O)C2=O</smiles>

Brown oil (58 mg, 87\%) obtained from the general procedure (4) using $1 \mathbf{b}$ (46 mg, $0.15 \mathrm{mmol}$ ) and 3-acetamidophenylboronic acid (54 $\mathrm{mg}, 0.30 \mathrm{mmol}$ ).

$\mathbf{R}_{\mathbf{f}}=0.09$ (petroleum ether/EtOAc 50:50).

SFC (Chiralpak ASH $s \mathrm{CO}_{2} / \mathrm{MeOH} 80: 20,2 \mathrm{~mL} / \mathrm{min}, \mathrm{P}=100$ bar): $\mathrm{Rt}=3.7 \mathrm{~min}$ (minor), 5.4 $\min$ (major).

$[\boldsymbol{\alpha}]_{\mathbf{D}}{ }^{\mathbf{2 0}}=+94\left(\mathrm{c}=1.5, \mathrm{CHCl}_{3}\right)$ for an enantiomeric excess of $86 \%$.

${ }^{1} \mathbf{H}$ NMR $\left(400 \mathrm{MHz}, \mathrm{CDCl}_{3}, \delta\right): 7.84-7.75(\mathrm{~m}, 1 \mathrm{H}), 7.70-7.64(\mathrm{~m}, 2 \mathrm{H}), 7.58-7.50(\mathrm{~m}, 2 \mathrm{H})$, $7.51-7.47(\mathrm{~m}, 1 \mathrm{H}), 7.38(\mathrm{t}, J=7.7 \mathrm{~Hz}, 1 \mathrm{H}), 7.30-7.27(\mathrm{~m}, 1 \mathrm{H}), 7.27-7.23(\mathrm{~m}, 1 \mathrm{H}), 2.98$ $(\mathrm{dd}, J=14.7,2.4 \mathrm{~Hz}, 1 \mathrm{H}), 2.61-2.49(\mathrm{~m}, 1 \mathrm{H}), 2.39(\mathrm{~d}, J=14.7 \mathrm{~Hz}, 1 \mathrm{H}), 2.36-2.28(\mathrm{~m}, 1 \mathrm{H})$, $2.24-2.06(\mathrm{~m}, 3 \mathrm{H}), 2.16(\mathrm{~s}, 3 \mathrm{H}), 1.98-1.90(\mathrm{~m}, 1 \mathrm{H}), 1.91-1.79(\mathrm{~m}, 1 \mathrm{H}), 0.98(\mathrm{~s}, 3 \mathrm{H})$.

${ }^{13}$ C NMR (101 MHz, $\left.\mathrm{CDCl}_{3}, \delta\right): 213.8,168.8,138.5,137.5,136.0,133.9,133.8,131.7,131.0$, 128.9 (q, $J=29.2 \mathrm{~Hz}), 127.4,126.6,126.1(\mathrm{q}, J=5.1 \mathrm{~Hz}), 124.2(\mathrm{q}, J=273.8 \mathrm{~Hz}), 123.8$, $121.1,115.0,78.2,53.5,37.1,36.7,24.5,20.9,15.7$.

${ }^{19}$ F NMR $\left(376 \mathrm{MHz}, \mathrm{CDCl}_{3}, \delta\right):-60.7$.

HRMS (ESI): Calculated for $\mathrm{C}_{25} \mathrm{H}_{24} \mathrm{~F}_{3} \mathrm{O}_{3} \mathrm{NNa}(\mathrm{M}+\mathrm{Na})^{+} 466.1606$, found 466.1600 .

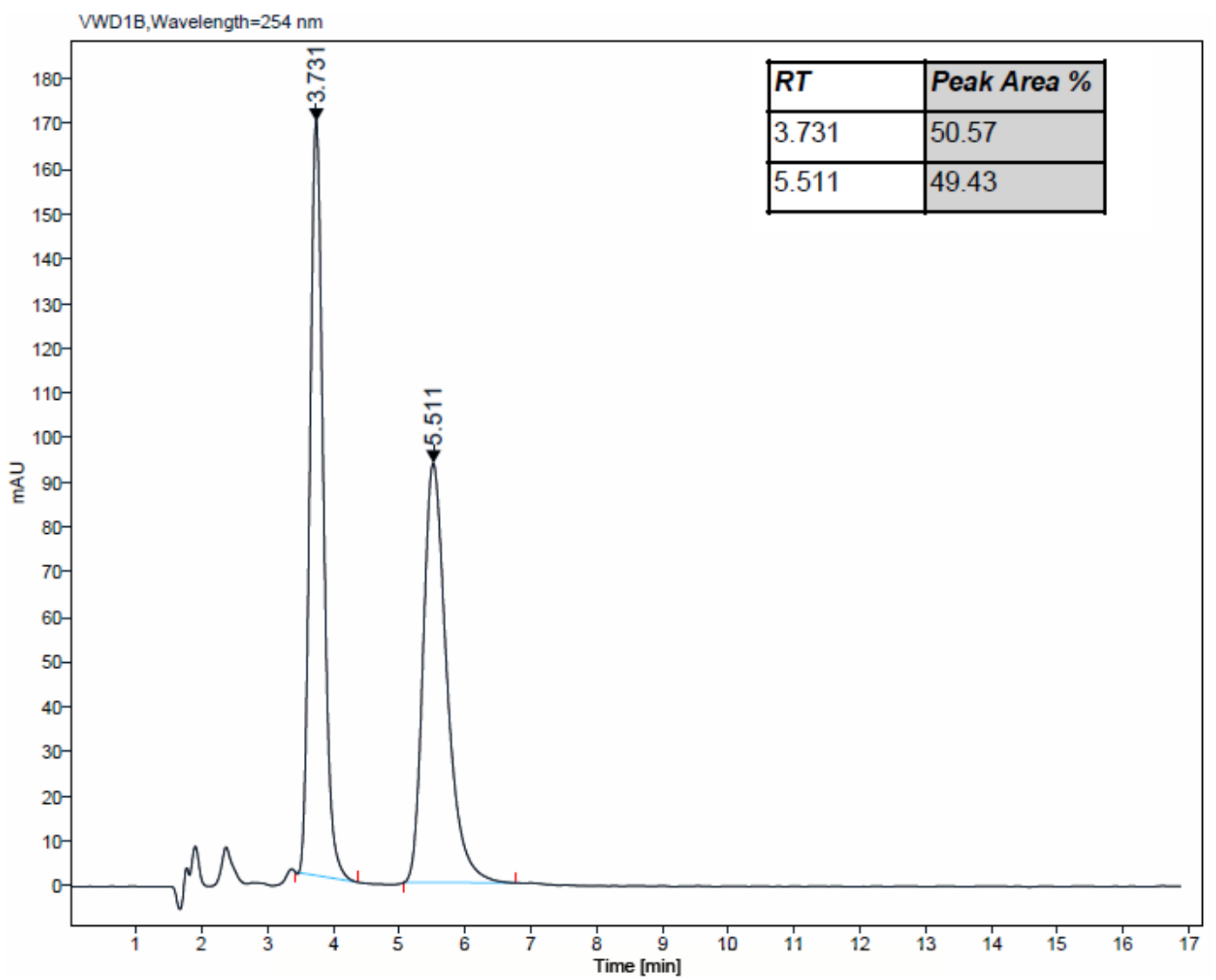




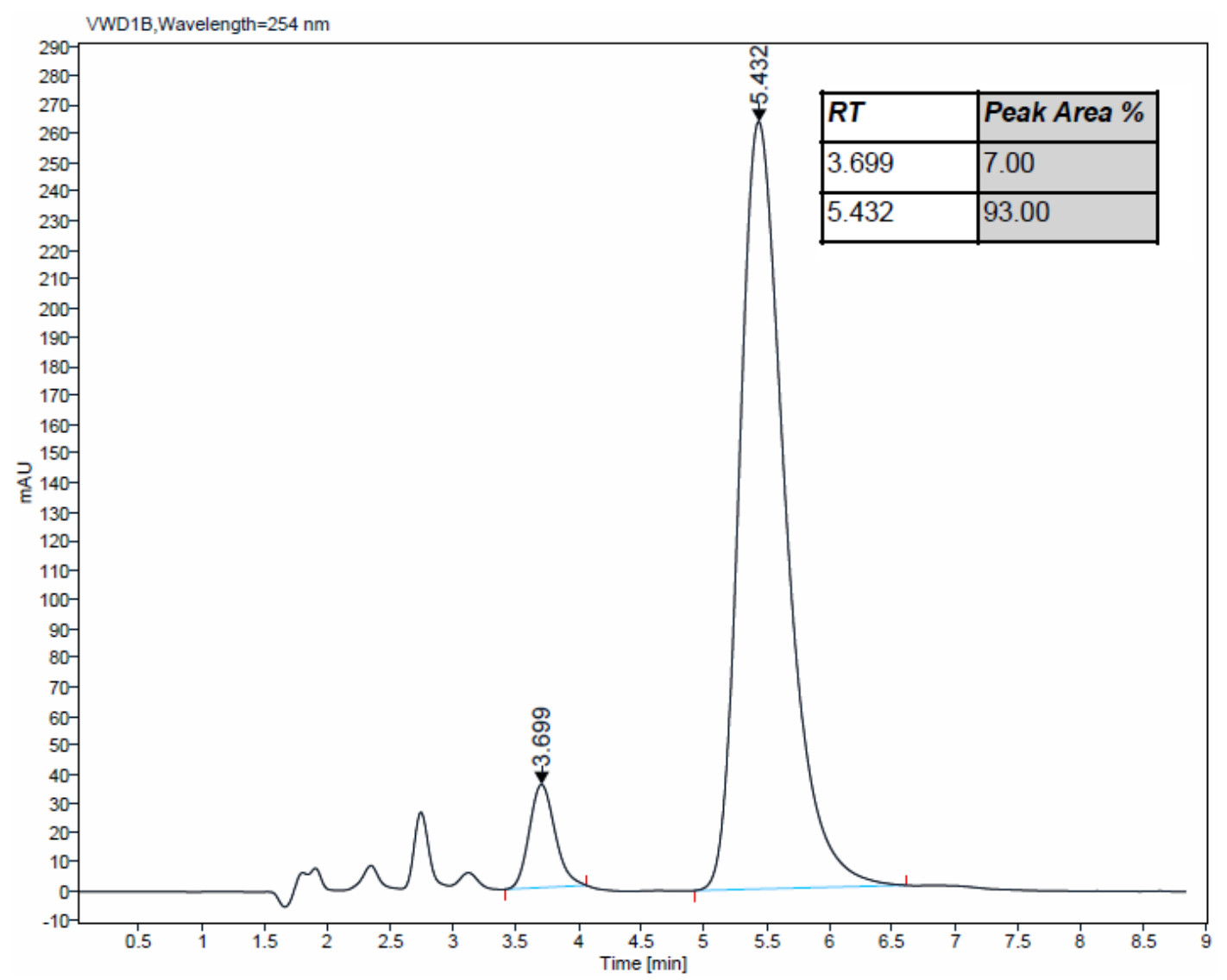

(E)-7-chloro-4a-hydroxy-10a-methyl-9-(2-(trifluoromethyl)benzylidene)-3,4,4a,9,10,10ahexahydrophenanthren-1(2H)-one (2p)

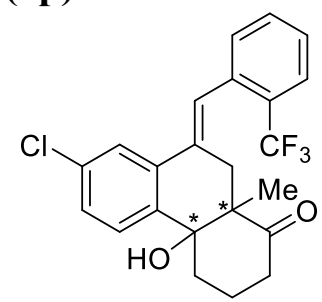

Orange oil (57 mg, 90\%) obtained from the general procedure (4) using $\mathbf{1 b}$ (46 mg, $0.15 \mathrm{mmol}$ ) and 3-chlorophenylboronic acid (47 $\mathrm{mg}, 0.30 \mathrm{mmol}$ ).

$\mathbf{R}_{\mathbf{f}}=0.27$ (petroleum ether/EtOAc 80:20).

SFC (Chiralpak ASH $s \mathrm{CO}_{2} / \mathrm{MeOH} 80: 20,2 \mathrm{~mL} / \mathrm{min}, \mathrm{P}=100$ bar): $\mathrm{Rt}=3.3 \mathrm{~min}$ (minor), 5.1 $\min$ (major).

$[\boldsymbol{\alpha}]_{\mathbf{D}}{ }^{\mathbf{2 0}}=+112\left(\mathrm{c}=1.3, \mathrm{CHCl}_{3}\right)$ for an enantiomeric excess of $82 \%$.

${ }^{1}$ H NMR $\left(400 \mathrm{MHz}, \mathrm{CDCl}_{3}, \delta\right): 7.74-7.64(\mathrm{~m}, 2 \mathrm{H}), 7.59(\mathrm{~d}, J=8.5 \mathrm{~Hz}, 1 \mathrm{H}), 7.56-7.49(\mathrm{~m}$, $1 \mathrm{H}), 7.46-7.38(\mathrm{~m}, 1 \mathrm{H}), 7.39-7.29(\mathrm{~m}, 2 \mathrm{H}), 7.26(\mathrm{~s}, 1 \mathrm{H}), 2.99(\mathrm{dd}, J=14.9,2.4 \mathrm{~Hz}, 1 \mathrm{H})$, $2.66-2.52(\mathrm{~m}, 1 \mathrm{H}), 2.41(\mathrm{dd}, J=14.9,0.9 \mathrm{~Hz}, 1 \mathrm{H}), 2.38-2.31(\mathrm{~m}, 1 \mathrm{H}), 2.29-2.13(\mathrm{~m}, 2 \mathrm{H})$, $1.93(\mathrm{~d}, J=2.1 \mathrm{~Hz}, 1 \mathrm{H}), 2.01-1.81(\mathrm{~m}, 2 \mathrm{H}), 1.00(\mathrm{~s}, 3 \mathrm{H})$.

${ }^{13} \mathbf{C}$ NMR $\left(101 \mathrm{MHz}, \mathrm{CDCl}_{3}, \delta\right): 213.2,140.9,135.6,134.8,133.9,133.2,131.7,130.9,129.0$ (q, $J=29.7 \mathrm{~Hz}), 129.0,127.6,127.5,126.2$ (q, $J=5.5 \mathrm{~Hz}), 124.6,124.1$ (q, $J=273.4 \mathrm{~Hz})$, $123.8,78.2,53.2,37.1,36.9,36.7,20.8,15.5$.

${ }^{19}$ F NMR $\left(376 \mathrm{MHz}, \mathrm{CDCl}_{3}, \delta\right):-60.8$.

HRMS (ESI): Calculated for $\mathrm{C}_{23} \mathrm{H}_{20} \mathrm{~F}_{3} \mathrm{O}_{2} \mathrm{ClNa}(\mathrm{M}+\mathrm{Na})^{+} 443.1002$, found 443.0997. 

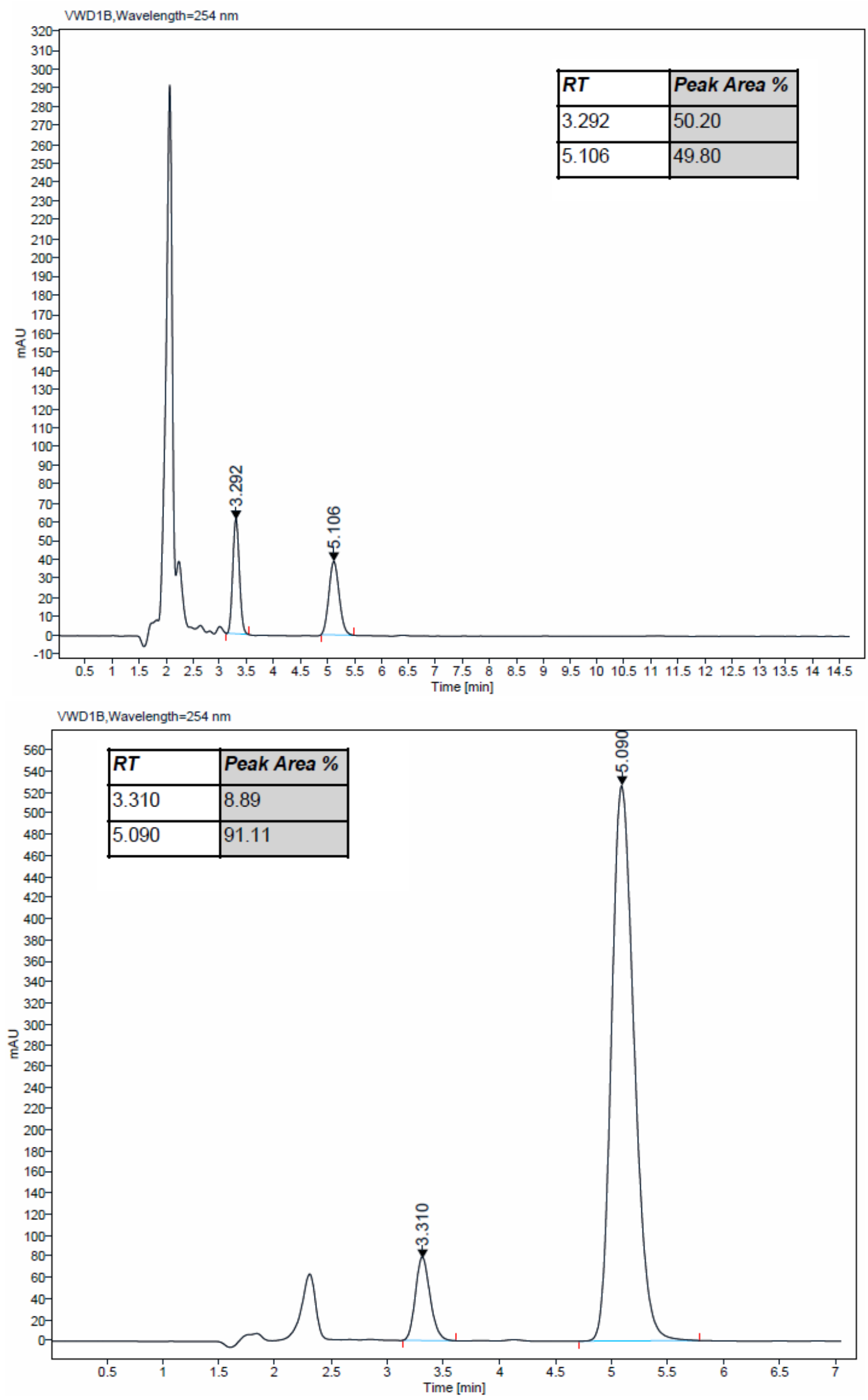

(E)-11b-hydroxy-4a-methyl-6-(2-(trifluoromethyl)benzylidene)-1,2,3,4a,5,6,8,11boctahydro-4H-naphtho[1,2-f]indol-4-one (2q) 


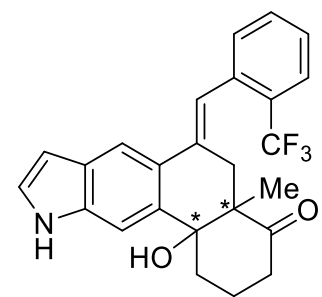

Brown oil (51 mg, 80\%) obtained from the general procedure (4) using $\mathbf{1 b}$ (46 mg, $0.15 \mathrm{mmol}$ ) and 5-indolylboronic acid (48 $\mathrm{mg}, 0.30 \mathrm{mmol}$ ).

$\mathbf{R}_{\mathbf{f}}=0.32$ (petroleum ether/EtOAc 50:50).

SFC (Chiralpak IE $s \mathrm{SO}_{2} / \mathrm{MeOH} 80: 20,2 \mathrm{~mL} / \mathrm{min}, \mathrm{P}=100$ bar): $\mathrm{Rt}=6.9 \mathrm{~min}$ (major), $18.9 \mathrm{~min}$ (minor).

$[\alpha]_{\mathbf{D}}{ }^{\mathbf{2 0}}=+62\left(\mathrm{c}=2.7, \mathrm{CHCl}_{3}\right)$ for an enantiomeric excess of $83 \%$.

${ }^{1}$ H NMR $\left(400 \mathrm{MHz}, \mathrm{CDCl}_{3}, \delta\right): 8.18(\mathrm{~s}, 1 \mathrm{H}), 8.03(\mathrm{~s}, 1 \mathrm{H}), 7.70(\mathrm{~d}, J=8.5 \mathrm{~Hz}, 1 \mathrm{H}), 7.68$ (s, $1 \mathrm{H}), 7.51(\mathrm{t}, J=7.6 \mathrm{~Hz}, 1 \mathrm{H}), 7.37(\mathrm{t}, J=7.6 \mathrm{~Hz}, 1 \mathrm{H}), 7.30(\mathrm{~d}, J=8.5 \mathrm{~Hz}, 2 \mathrm{H}), 7.26-7.23(\mathrm{~m}$, $1 \mathrm{H}), 6.71-6.52(\mathrm{~m}, 1 \mathrm{H}), 3.08(\mathrm{dd}, J=14.9,1.6 \mathrm{~Hz}, 1 \mathrm{H}), 2.69-2.58(\mathrm{~m}, 1 \mathrm{H}), 2.49$ (dd, $J=$ $14.9,1.0 \mathrm{~Hz}, 1 \mathrm{H}), 2.40-2.28(\mathrm{~m}, 2 \mathrm{H}), 2.27-2.15(\mathrm{~m}, 1 \mathrm{H}), 2.00(\mathrm{~d}, J=2.1 \mathrm{~Hz}, 1 \mathrm{H}), 2.02-$ $1.92(\mathrm{~m}, 1 \mathrm{H}), 1.94-1.86(\mathrm{~m}, 1 \mathrm{H}), 1.02(\mathrm{~s}, 3 \mathrm{H})$.

${ }^{13} \mathbf{C}$ NMR $\left(101 \mathrm{MHz}, \mathrm{CDCl}_{3}, \delta\right): 214.2,137.9,136.8,136.6,135.4,131.6,131.2,129.1$ (q, $J=$ $29.5 \mathrm{~Hz}), 127.8,127.0,126.2,126.0(\mathrm{q}, J=5.3 \mathrm{~Hz}), 125.7,124.3(\mathrm{q}, J=274.2 \mathrm{~Hz}), 121.4$, $116.4,107.5,102.9,79.4,53.9,37.7,37.3,37.0,21.2,15.7$.

${ }^{19}$ F NMR $\left(376 \mathrm{MHz}, \mathrm{CDCl}_{3}, \delta\right):-60.8$.

HRMS (ESI): Calculated for $\mathrm{C}_{25} \mathrm{H}_{22} \mathrm{~F}_{3} \mathrm{O}_{2} \mathrm{NNa}(\mathrm{M}+\mathrm{Na})^{+} 448.1500$, found 448.1495.

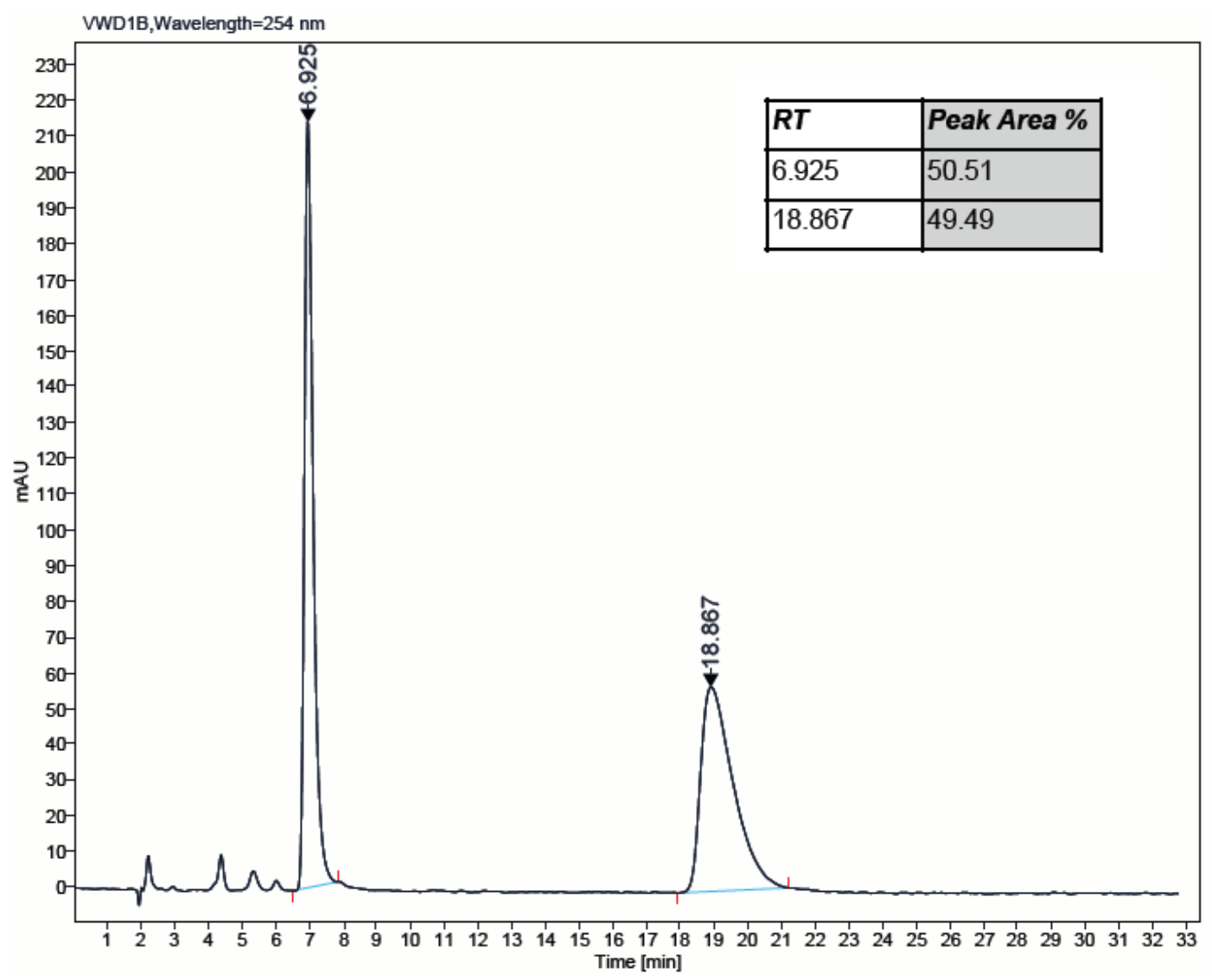




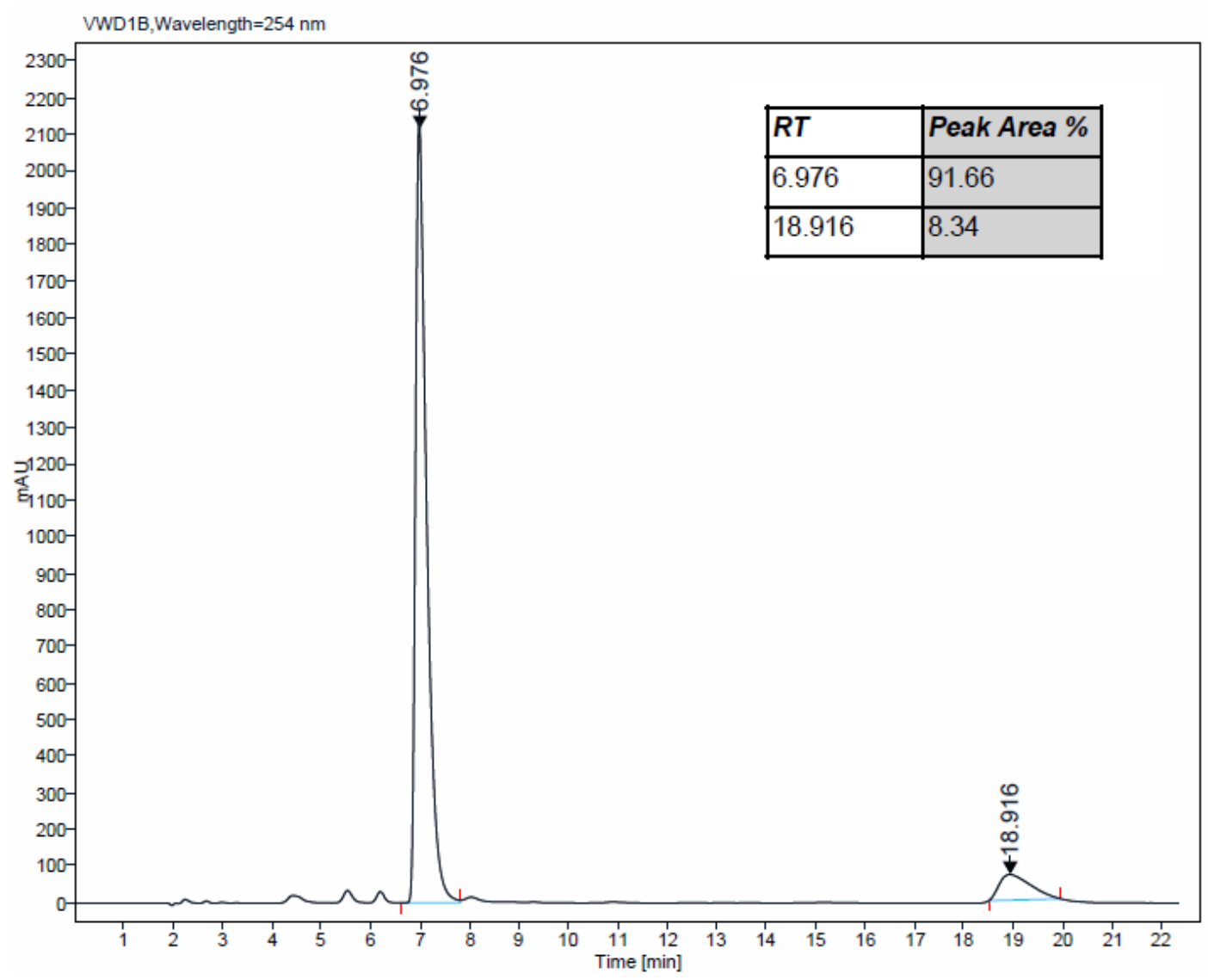

(E)-10a-benzyl-4a-hydroxy-6-methoxy-9-(2-(trifluoromethyl)benzylidene)$3,4,4 a, 9,10,10 a-h e x a h y d r o p h e n a n t h r e n-1(2 H)$-one $(2 r)$<smiles>COc1ccc2c(c1)C1(O)CCCC(=O)C1(Br)CC2=Cc1ccccc1F</smiles>

Orange oil (63 mg, 86\%) obtained from the general procedure (4) using 1c (58 mg, $0.15 \mathrm{mmol}$ ) and 4-methoxyphenylboronic acid (46 mg, $0.30 \mathrm{mmol}$ ).

$\mathbf{R}_{\mathbf{f}}=0.29$ (petroleum ether/EtOAc 80:20).

SFC (Chiralpak ASH $s \mathrm{CO}_{2} / \mathrm{MeOH} 80: 20,2 \mathrm{~mL} / \mathrm{min}, \mathrm{P}=100$ bar): $\mathrm{Rt}=6.8 \mathrm{~min}$ (minor), 8.5 min (major).

$[\boldsymbol{\alpha}]_{\mathbf{D}}{ }^{\mathbf{2 0}}=-283\left(\mathrm{c}=1.1, \mathrm{CHCl}_{3}\right)$ for an enantiomeric excess of $89 \%$.

${ }^{1} \mathbf{H}$ NMR $\left(400 \mathrm{MHz}, \mathrm{CDCl}_{3}, \delta\right): 7.79(\mathrm{~d}, J=8.8 \mathrm{~Hz}, 1 \mathrm{H}), 7.72-7.61(\mathrm{~m}, 1 \mathrm{H}), 7.37-7.28(\mathrm{~m}$, $3 \mathrm{H}), 7.23-7.18(\mathrm{~m}, 1 \mathrm{H}), 7.18-7.13(\mathrm{~m}, 2 \mathrm{H}), 7.11(\mathrm{~d}, J=2.8 \mathrm{~Hz}, 1 \mathrm{H}), 7.05-6.98(\mathrm{~m}, 2 \mathrm{H})$, $6.95(\mathrm{dd}, J=8.8,2.8 \mathrm{~Hz}, 1 \mathrm{H}), 6.88(\mathrm{~d}, J=7.3 \mathrm{~Hz}, 1 \mathrm{H}), 3.84(\mathrm{~s}, 3 \mathrm{H}), 3.08(\mathrm{~d}, J=13.7 \mathrm{~Hz}, 1 \mathrm{H})$, $2.90-2.83(\mathrm{~m}, 1 \mathrm{H}), 2.81(\mathrm{~d}, J=13.7 \mathrm{~Hz}, 1 \mathrm{H}), 2.67-2.55(\mathrm{~m}, 1 \mathrm{H}), 2.49-2.35(\mathrm{~m}, 2 \mathrm{H}), 2.26$ $-2.08(\mathrm{~m}, 2 \mathrm{H}), 1.93(\mathrm{~d}, J=1.9 \mathrm{~Hz}, 1 \mathrm{H}), 1.91-1.70(\mathrm{~m}, 2 \mathrm{H})$.

${ }^{13} \mathrm{C}$ NMR (101 MHz, $\left.\mathrm{CDCl}_{3}, \delta\right): 212.8,160.2,143.7,138.5,136.5,132.6,131.6,131.2(2 \mathrm{C})$, $130.7,128.7(\mathrm{q}, J=29.4 \mathrm{~Hz}), 128.3(2 \mathrm{C}), 126.8,126.5,126.2,125.9$ (q, $J=5.5 \mathrm{~Hz}), 125.7$, $124.3(\mathrm{q}, J=273.4 \mathrm{~Hz}), 120.9,114.5,109.2,79.6,58.0,55.5,36.9,35.9,35.9,33.6,20.6$.

${ }^{19}$ F NMR $\left(376 \mathrm{MHz}, \mathrm{CDCl}_{3}, \delta\right):-61.2$.

HRMS (ESI): Calculated for $\mathrm{C}_{30} \mathrm{H}_{27} \mathrm{~F}_{3} \mathrm{O}_{3} \mathrm{Na}(\mathrm{M}+\mathrm{Na})^{+}$515.1810, found 515.1804. 

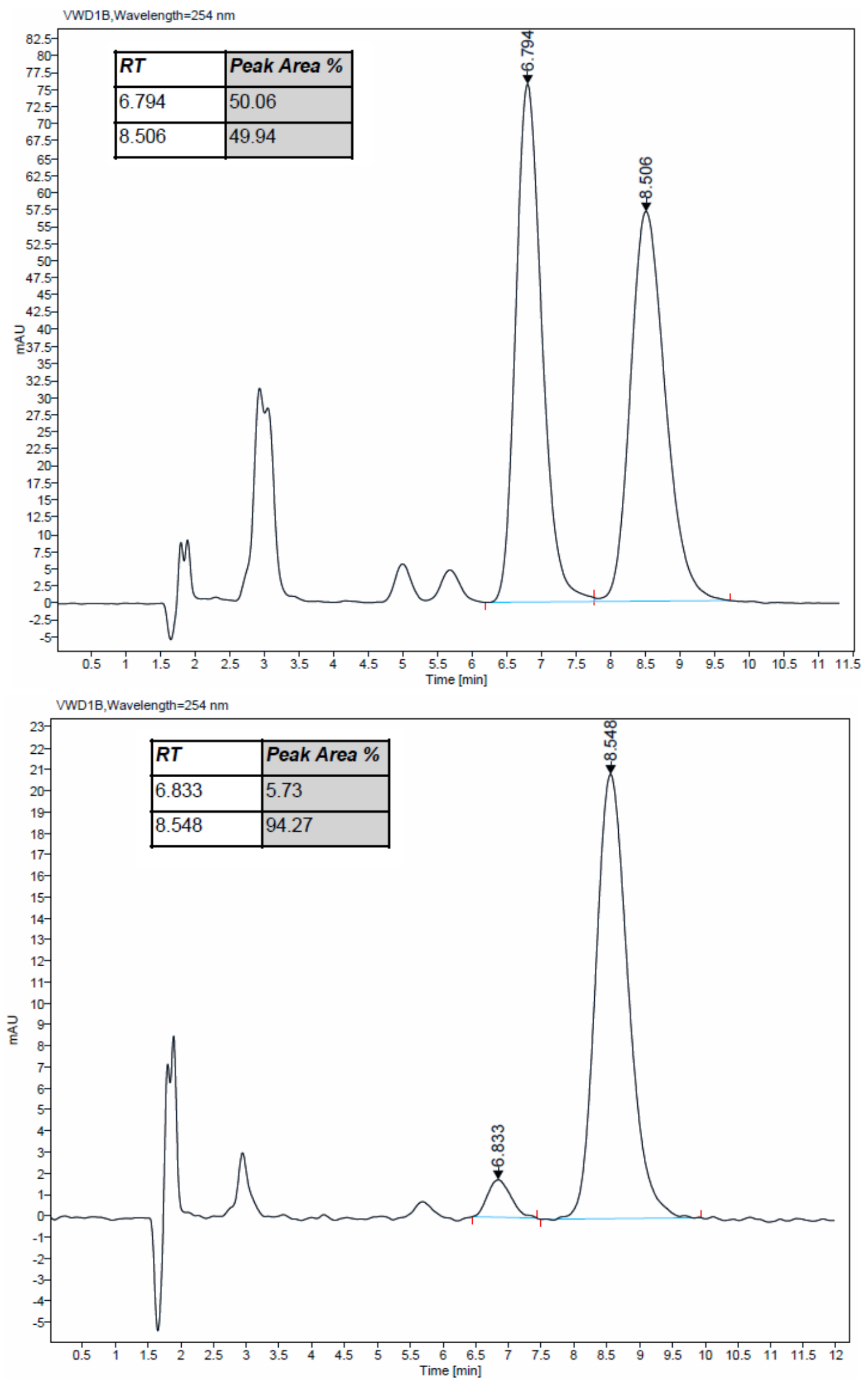

(E)-10a-benzyl-6-(tert-butyl)-4a-hydroxy-9-(2-(trifluoromethyl)benzylidene)$3,4,4 a, 9,10,10 a-h e x a h y d r o p h e n a n t h r e n-1(2 H)$-one $(2 s)$ 
<smiles>CC(C)(C)c1ccc2c(c1)C1(O)CCCC(=O)C1(Br)C2=Cc1ccccc1F</smiles>

Yellow oil (68 mg, 87\%) obtained from the general procedure (4) using 1c (58 mg, $0.15 \mathrm{mmol}$ ) and 4-tert-butylphenylboronic acid (54 $\mathrm{mg}, 0.30 \mathrm{mmol}$ ).

$\mathbf{R}_{\mathbf{f}}=0.41$ (petroleum ether/EtOAc 80:20).

SFC (Chiralpak $\mathrm{ADH} s \mathrm{CO}_{2} / \mathrm{MeOH} 80: 20,2 \mathrm{~mL} / \mathrm{min}, \mathrm{P}=100$ bar): $\mathrm{Rt}=4.5 \mathrm{~min}$ (major), 6.7 $\min$ (minor).

$[\alpha]_{\mathbf{D}}{ }^{20}=-252\left(\mathrm{c}=0.9, \mathrm{CHCl}_{3}\right)$ for an enantiomeric excess of $90 \%$.

${ }^{1} \mathbf{H}$ NMR $\left(400 \mathrm{MHz}, \mathrm{CDCl}_{3}, \delta\right): 7.80(\mathrm{~d}, J=8.4 \mathrm{~Hz}, 1 \mathrm{H}), 7.70-7.63(\mathrm{~m}, 1 \mathrm{H}), 7.59(\mathrm{~d}, J=2.1$ $\mathrm{Hz}, 1 \mathrm{H}), 7.43(\mathrm{dd}, J=8.4,2.1 \mathrm{~Hz}, 1 \mathrm{H}), 7.40-7.29(\mathrm{~m}, 3 \mathrm{H}), 7.19-7.12(\mathrm{~m}, 3 \mathrm{H}), 6.99-6.93$ (m, 3H), 3.00 (d, $J=13.7 \mathrm{~Hz}, 1 \mathrm{H}), 2.92$ (d, $J=13.7 \mathrm{~Hz}, 1 \mathrm{H}), 2.89$ (dd, $J=17.1,2.2 \mathrm{~Hz}, 1 \mathrm{H})$, $2.67-2.55(\mathrm{~m}, 1 \mathrm{H}), 2.53-2.35(\mathrm{~m}, 2 \mathrm{H}), 2.27-2.10(\mathrm{~m}, 2 \mathrm{H}), 1.92(\mathrm{~d}, J=2.0 \mathrm{~Hz}, 1 \mathrm{H}), 1.92-$ $1.86(\mathrm{~m}, 1 \mathrm{H}), 1.83-1.76(\mathrm{~m}, 1 \mathrm{H}), 1.38(\mathrm{~s}, 9 \mathrm{H})$.

${ }^{13} \mathrm{C}$ NMR (101 MHz, $\left.\mathrm{CDCl}_{3}, \delta\right): 212.8,151.8,141.4,138.5,136.4,133.0,131.6,131.2(2 \mathrm{C})$, $130.7(2 \mathrm{C}), 128.7$ (q, $J=29.3 \mathrm{~Hz}), 128.3(2 \mathrm{C}), 127.0,126.6,125.9$ (q, $J=5.3 \mathrm{~Hz}), 125.0,124.2$ $(\mathrm{q}, J=273.8 \mathrm{~Hz}), 123.8,122.2,121.4,79.5,58.1,36.9,36.3,35.8,34.9,33.8,31.3$ (3C), 20.5 . ${ }^{19}$ F NMR $\left(376 \mathrm{MHz}, \mathrm{CDCl}_{3}, \delta\right):-61.2$.

HRMS (ESI): Calculated for $\mathrm{C}_{33} \mathrm{H}_{33} \mathrm{~F}_{3} \mathrm{O}_{2} \mathrm{Na}(\mathrm{M}+\mathrm{Na})^{+}$541.2330, found 541.2323.

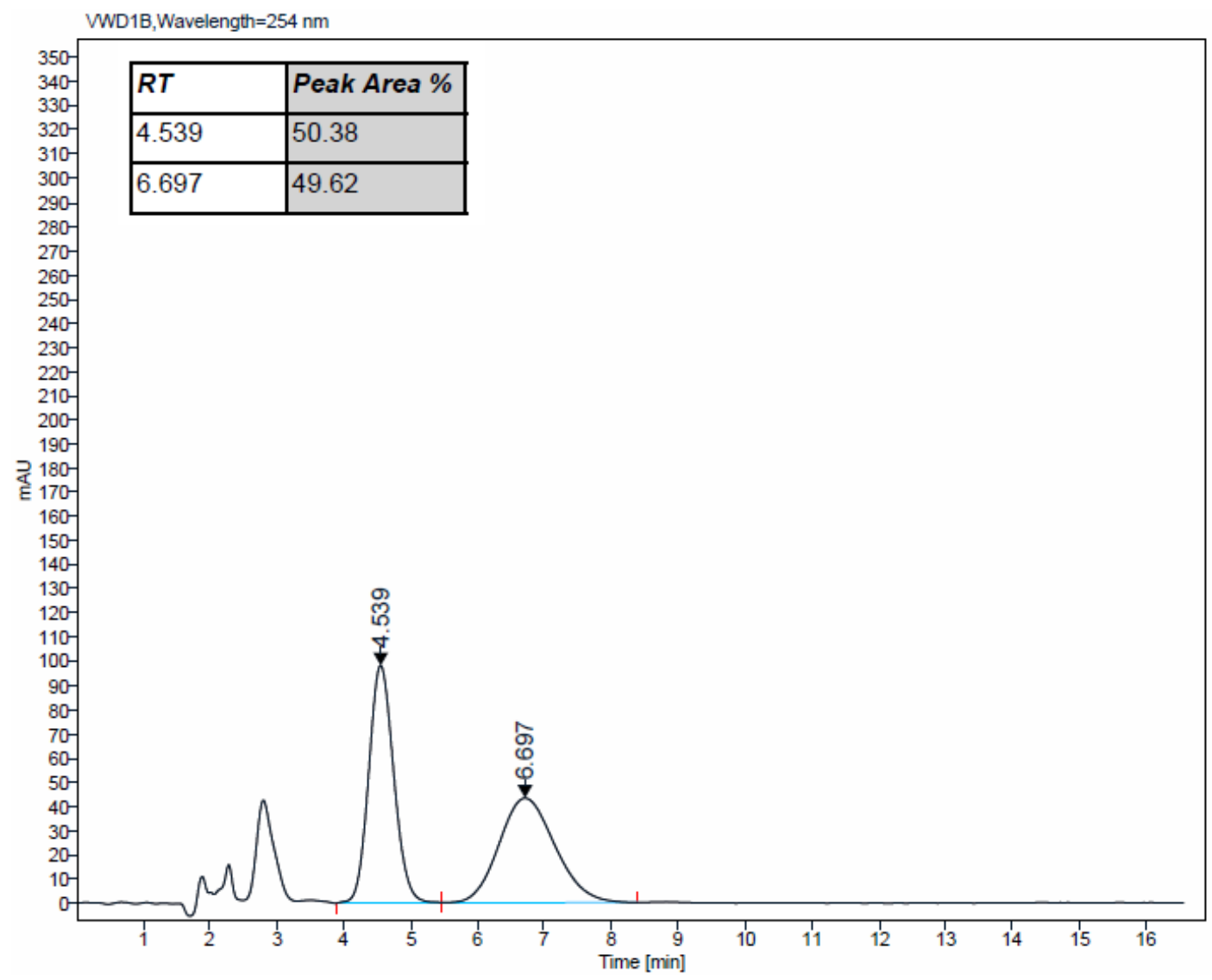




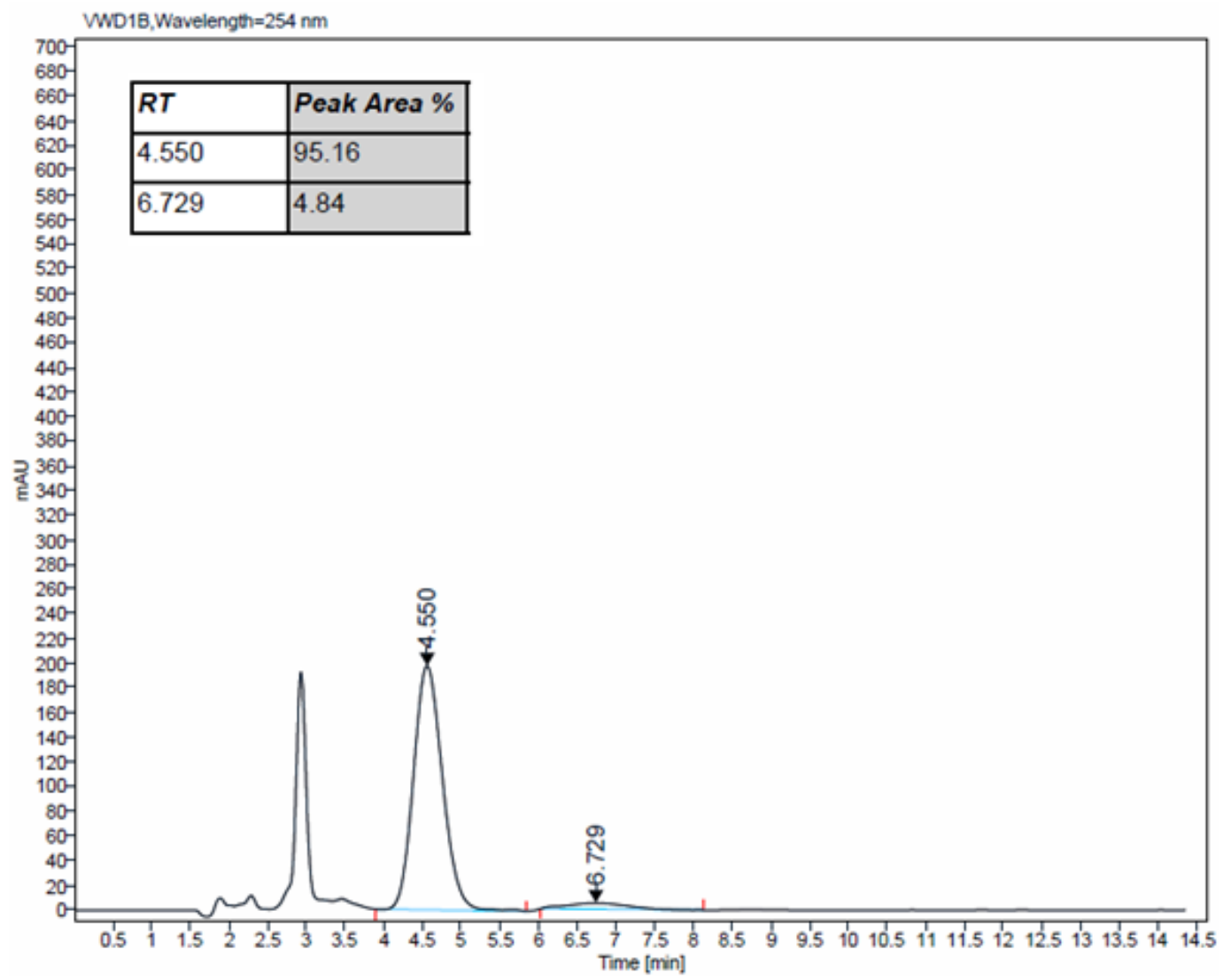

(E)-10a-benzyl-4a-hydroxy-6-methyl-9-(2-(trifluoromethyl)benzylidene)-3,4,4a,9,10,10ahexahydrophenanthren-1(2H)-one (2t)<smiles>Cc1ccc2c(c1)C1(O)CCCC(=O)C1(Br)C2=Cc1ccccc1F</smiles>

Colorless oil (64 mg, 89\%) obtained from the general procedure (4) using 1c (58 mg, 0.15 $\mathrm{mmol}$ ) and 4-tolylboronic acid (41 $\mathrm{mg}, 0.30 \mathrm{mmol})$.

$\mathbf{R}_{\mathbf{f}}=0.46$ (petroleum ether/EtOAc 80:20).

SFC (Chiralpak ADH $s \mathrm{CO}_{2} / \mathrm{MeOH} 80: 20,2 \mathrm{~mL} / \mathrm{min}, \mathrm{P}=100 \mathrm{bar}$ ): $\mathrm{Rt}=6.2 \mathrm{~min}$ (major), 9.5 $\min$ (minor).

$[\boldsymbol{\alpha}]_{\mathbf{D}}{ }^{\mathbf{2 0}}=-243\left(\mathrm{c}=1.3, \mathrm{CHCl}_{3}\right)$ for an enantiomeric excess of $91 \%$.

${ }^{1} \mathbf{H}$ NMR $\left(400 \mathrm{MHz}, \mathrm{CDCl}_{3}, \delta\right): 7.75(\mathrm{~d}, J=8.1 \mathrm{~Hz}, 1 \mathrm{H}), 7.69-7.61(\mathrm{~m}, 1 \mathrm{H}), 7.42-7.39$ (m, $1 \mathrm{H}), 7.35-7.29(\mathrm{~m}, 2 \mathrm{H}), 7.27-7.24(\mathrm{~m}, 1 \mathrm{H}), 7.21(\mathrm{dd}, J=8.1,1.9 \mathrm{~Hz}, 1 \mathrm{H}), 7.18-7.12(\mathrm{~m}$, $3 \mathrm{H}), 7.06-6.93(\mathrm{~m}, 2 \mathrm{H}), 6.91-6.83(\mathrm{~m}, 1 \mathrm{H}), 3.14(\mathrm{~d}, J=13.7 \mathrm{~Hz}, 1 \mathrm{H}), 2.84(\mathrm{dd}, J=17.1,2.5$ $\mathrm{Hz}, 1 \mathrm{H}), 2.74(\mathrm{~d}, J=13.7 \mathrm{~Hz}, 1 \mathrm{H}), 2.60(\mathrm{ddd}, J=15.0,11.1,6.8 \mathrm{~Hz}, 1 \mathrm{H}), 2.52-2.34(\mathrm{~m}, 2 \mathrm{H})$, $2.42(\mathrm{~s}, 3 \mathrm{H}), 2.28-2.10(\mathrm{~m}, 2 \mathrm{H}), 1.94-1.87(\mathrm{~m}, 1 \mathrm{H}), 1.92(\mathrm{~d}, J=1.8 \mathrm{~Hz}, 1 \mathrm{H}), 1.85-1.70(\mathrm{~m}$, $1 \mathrm{H})$.

${ }^{13}$ C NMR (101 MHz, $\left.\mathrm{CDCl}_{3}, \delta\right): 212.8,141.7,138.7,138.5,136.4,133.0,131.5,131.1(2 \mathrm{C})$, $130.8,130.7,128.9,128.2,126.9,128.6(\mathrm{q}, J=29.6 \mathrm{~Hz}), 126.4(2 \mathrm{C}), 125.8(\mathrm{q}, J=5.4 \mathrm{~Hz})$, $125.2,124.3(\mathrm{q}, J=274.0 \mathrm{~Hz}), 124.0,121.9,79.4,58.0,37.0,36.1,35.7,32.9,21.5,20.7$.

${ }^{19}$ F NMR $\left(376 \mathrm{MHz}, \mathrm{CDCl}_{3}, \delta\right):-61.2$.

HRMS (ESI): Calculated for $\mathrm{C}_{30} \mathrm{H}_{27} \mathrm{~F}_{3} \mathrm{O}_{2} \mathrm{Na}(\mathrm{M}+\mathrm{Na})^{+} 499.1861$, found 499.1853 . 

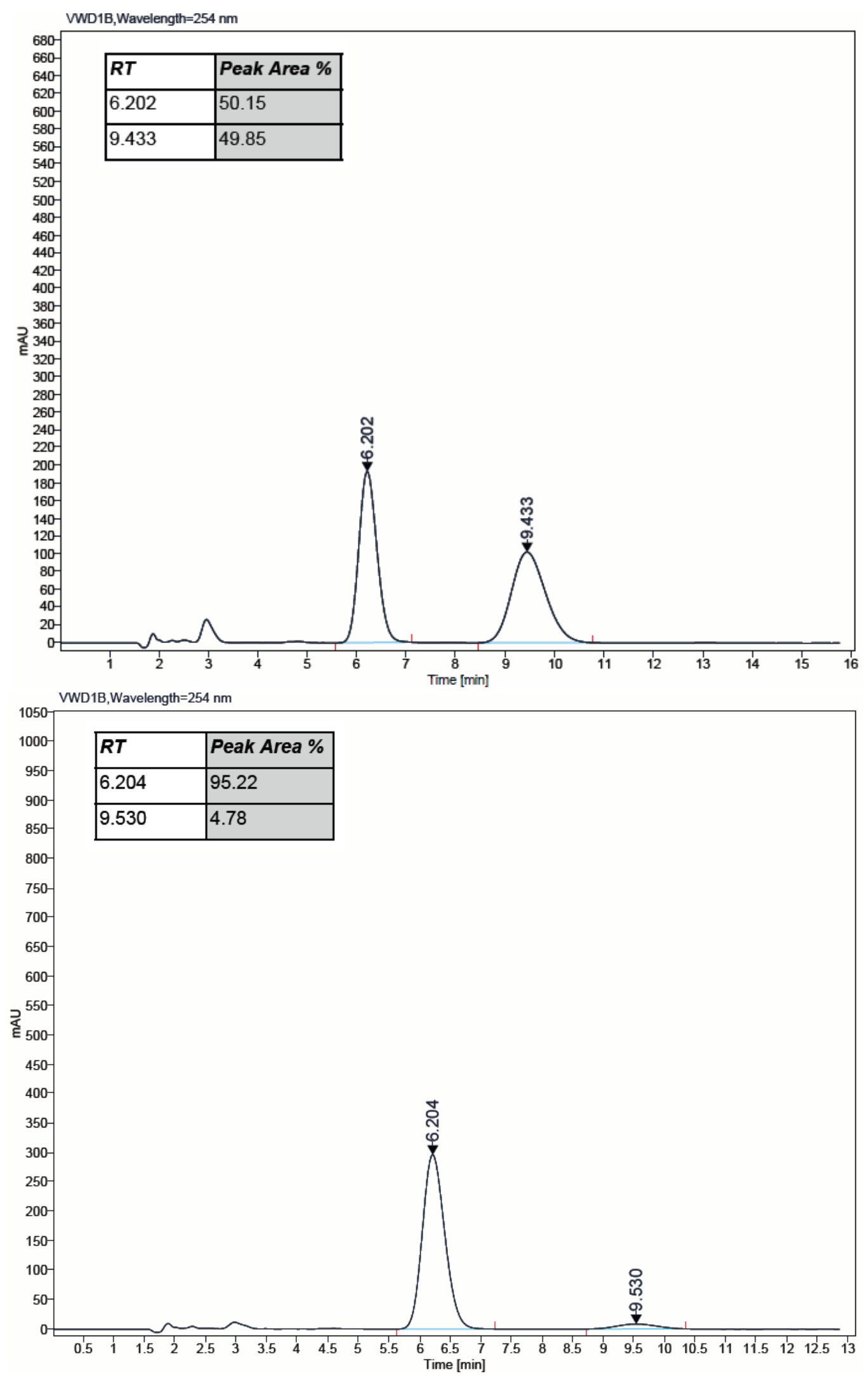

(E)-10a-benzyl-6-bromo-4a-hydroxy-9-(2-(trifluoromethyl)benzylidene)-3,4,4a,9,10,10ahexahydrophenanthren-1(2H)-one $(2 \mathrm{u})$ 
<smiles>O=C1CCCCC1(O)C1(Br)CC(=Cc2ccccc2F)c2ccc(Br)cc21</smiles>

Yellow oil (66 mg, 81\%) obtained from the general procedure (4) using 1c (58 mg, $0.15 \mathrm{mmol}$ ) and 4-bromophenylboronic acid (60 $\mathrm{mg}, 0.30 \mathrm{mmol})$.

$\mathbf{R}_{\mathbf{f}}=0.38$ (petroleum ether/EtOAc 80:20).

SFC (Chiralpak ADH $s \mathrm{CO}_{2} / \mathrm{MeOH} 80: 20,2 \mathrm{~mL} / \mathrm{min}, \mathrm{P}=100$ bar): $\mathrm{Rt}=8.0 \mathrm{~min}$ (major), 16.1 $\min$ (minor).

$[\boldsymbol{\alpha}]_{\mathbf{D}^{20}}{ }^{\mathbf{2 0}}=-268\left(\mathrm{c}=1.2, \mathrm{CHCl}_{3}\right)$ for an enantiomeric excess of $90 \%$.

${ }^{1} \mathbf{H}$ NMR $\left(400 \mathrm{MHz}, \mathrm{CDCl}_{3}, \delta\right): 7.74(\mathrm{~d}, J=2.2 \mathrm{~Hz}, 1 \mathrm{H}), 7.70(\mathrm{~d}, J=8.5 \mathrm{~Hz}, 1 \mathrm{H}), 7.68-7.56$ $(\mathrm{m}, 2 \mathrm{H}), 7.51(\mathrm{dd}, J=8.5,2.2 \mathrm{~Hz}, 1 \mathrm{H}), 7.39-7.32(\mathrm{~m}, 2 \mathrm{H}), 7.31-7.28(\mathrm{~m}, 1 \mathrm{H}), 7.22-7.16$ $(\mathrm{m}, 3 \mathrm{H}), 7.02-6.96(\mathrm{~m}, 2 \mathrm{H}), 6.88-6.82(\mathrm{~m}, 1 \mathrm{H}), 3.08(\mathrm{~d}, J=13.8 \mathrm{~Hz}, 1 \mathrm{H}), 2.88-2.79(\mathrm{~m}$, $1 \mathrm{H}), 2.78(\mathrm{~d}, J=13.8 \mathrm{~Hz}, 1 \mathrm{H}), 2.64-2.53(\mathrm{~m}, 1 \mathrm{H}), 2.49-2.36(\mathrm{~m}, 2 \mathrm{H}), 2.19-2.10(\mathrm{~m}, 2 \mathrm{H})$, $1.95(\mathrm{~d}, J=1.1 \mathrm{~Hz}, 1 \mathrm{H}), 1.91-1.76(\mathrm{~m}, 1 \mathrm{H})$.

${ }^{13} \mathbf{C}$ NMR $\left(101 \mathrm{MHz}, \mathrm{CDCl}_{3}, \delta\right): 212.4,143.9,138.1,136.1,135.8,132.5,132.4,131.7,131.1$ (2C), 130.5, 128.6 (q, $J=28.8 \mathrm{~Hz}), 128.4(2 \mathrm{C}), 128.1,127.3,126.6,126.0$ (q, $J=5.3,4.5 \mathrm{~Hz})$, $125.8,124.2(\mathrm{q}, J=273.6 \mathrm{~Hz}), 123.6,123.0,79.2,57.6,36.9,36.1,35.7,33.0,20.6$.

${ }^{19}$ F NMR $\left(376 \mathrm{MHz}, \mathrm{CDCl}_{3}, \delta\right):-61.2$.

HRMS (ESI): Calculated for $\mathrm{C}_{29} \mathrm{H}_{24} \mathrm{BrF}_{3} \mathrm{O}_{2} \mathrm{Na}(\mathrm{M}+\mathrm{Na})^{+}$563.0809, found 563.0803.

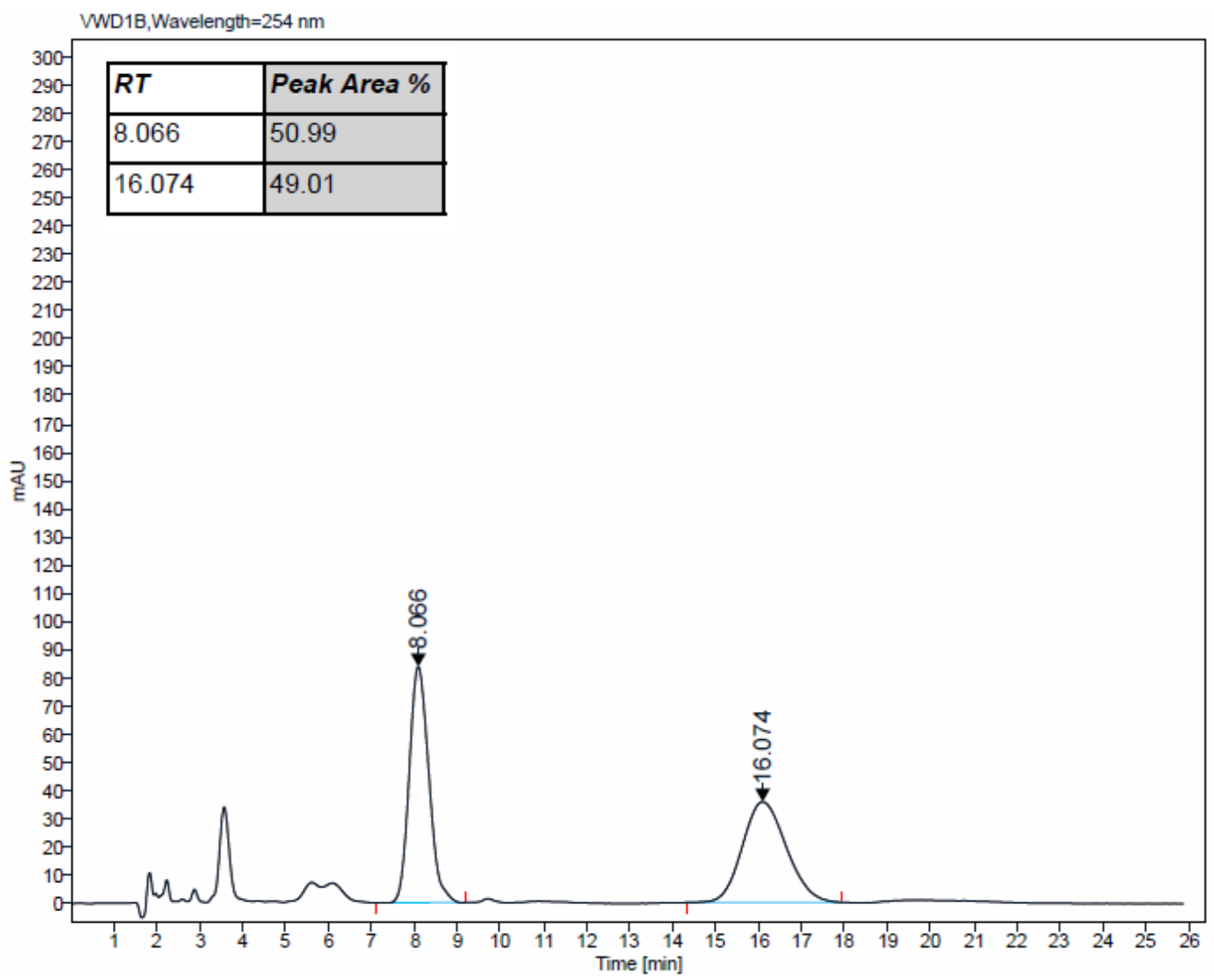




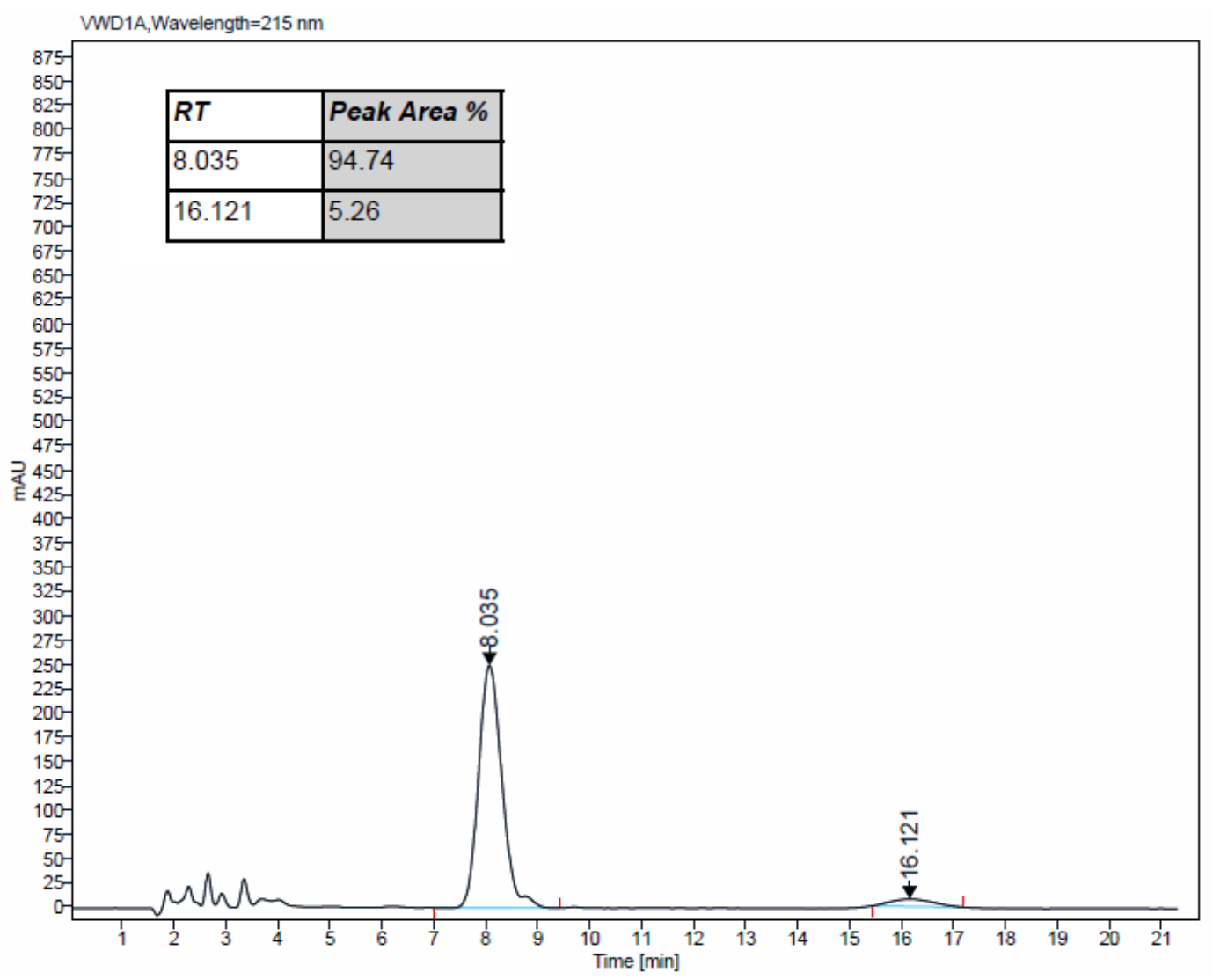

(E)-10a-benzyl-4a-hydroxy-7-methoxy-9-(2-(trifluoromethyl)benzylidene)$3,4,4 a, 9,10,10 a-h e x a h y d r o p h e n a n t h r e n-1(2 H)-o n e ~(2 v)$<smiles>COc1ccc2c(c1)/C(=C/c1ccccc1C(F)(F)F)CC1(O)C(=O)CCCC21O</smiles>

Yellow oil (67 mg, 91\%) obtained from the general procedure (4) using 1c (58 mg, $0.15 \mathrm{mmol})$ and 3-methoxyphenylboronic acid (46 $\mathrm{mg}, 0.30 \mathrm{mmol})$.

$\mathbf{R}_{\mathbf{f}}=0.23$ (petroleum ether/EtOAc 80:20).

SFC (Chiralpak ADH $s \mathrm{CO}_{2} / \mathrm{MeOH} 90: 10,2 \mathrm{~mL} / \mathrm{min}, \mathrm{P}=100$ bar): $\mathrm{Rt}=16.8 \mathrm{~min}$ (minor), 19.4 $\min$ (major).

$[\boldsymbol{\alpha}]_{\mathbf{D}}{ }^{\mathbf{2 0}}=-258\left(\mathrm{c}=0.9, \mathrm{CHCl}_{3}\right)$ for an enantiomeric excess of $92 \%$.

${ }^{1} \mathbf{H}$ NMR $\left(400 \mathrm{MHz}, \mathrm{CDCl}_{3}, \delta\right): 7.67-7.61(\mathrm{~m}, 1 \mathrm{H}), 7.51(\mathrm{~d}, J=8.7 \mathrm{~Hz}, 1 \mathrm{H}), 7.37-7.31(\mathrm{~m}$, $3 \mathrm{H}), 7.31-7.26(\mathrm{~m}, 1 \mathrm{H}), 7.19-7.13(\mathrm{~m}, 3 \mathrm{H}), 7.04-6.92(\mathrm{~m}, 4 \mathrm{H}), 3.90(\mathrm{~s}, 3 \mathrm{H}), 3.14(\mathrm{~d}, J=$ $13.7 \mathrm{~Hz}, 1 \mathrm{H}), 2.86(\mathrm{dd}, J=16.9,2.1 \mathrm{~Hz}, 1 \mathrm{H}), 2.85(\mathrm{~d}, J=13.7 \mathrm{~Hz}, 1 \mathrm{H}), 2.59-2.43(\mathrm{~m}, 2 \mathrm{H})$, $2.37(\mathrm{dd}, J=16.8,2.0 \mathrm{~Hz}, 1 \mathrm{H}), 2.28-2.19(\mathrm{~m}, 1 \mathrm{H}), 2.16-2.05(\mathrm{~m}, 1 \mathrm{H}), 1.97(\mathrm{dt}, J=14.0,4.7$ $\mathrm{Hz}, 1 \mathrm{H}), 1.92(\mathrm{~d}, J=1.8 \mathrm{~Hz}, 1 \mathrm{H}), 1.79-1.67(\mathrm{~m}, 1 \mathrm{H})$.

${ }^{13}$ C NMR (101 MHz, $\left.\mathrm{CDCl}_{3}, \delta\right): 212.5,159.2,138.3,136.2,135.0,134.1,133.4,131.6,131.0$ (2C), 130.7, $128.6(\mathrm{q}, J=29.3 \mathrm{~Hz}), 128.3(2 \mathrm{C}), 127.0,126.5(2 \mathrm{C}), 125.8$ (q, $J=5.0 \mathrm{~Hz}), 124.2$ $(\mathrm{q}, J=273.7 \mathrm{~Hz}), 122.9,114.8,108.7,78.7,58.2,55.4,37.1,36.6,35.4,32.6,20.5$.

${ }^{19}$ F NMR $\left(376 \mathrm{MHz}, \mathrm{CDCl}_{3}, \delta\right):-61.2$.

HRMS (ESI): Calculated for $\mathrm{C}_{30} \mathrm{H}_{27} \mathrm{~F}_{3} \mathrm{O}_{3} \mathrm{Na}(\mathrm{M}+\mathrm{Na})^{+}$515.1810, found 515.1804. 

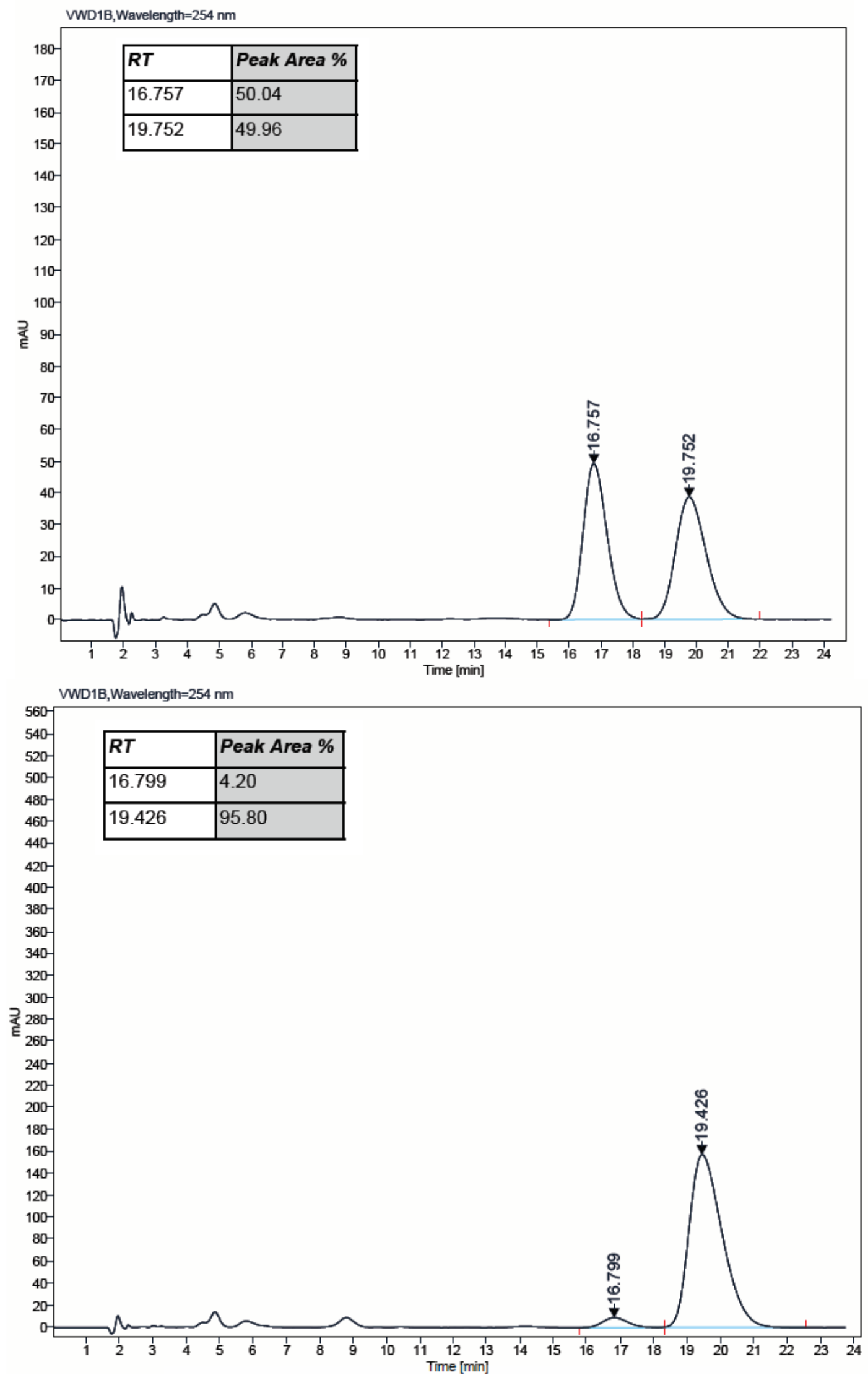

(E)- $N$-(8a-benzyl-4b-hydroxy-8-oxo-10-(2-(trifluoromethyl)benzylidene)4b,5,6,7,8,8a,9,10-octahydrophenanthren-2-yl)acetamide (2w) 
<smiles>CC(=O)Nc1ccc2c(c1)C(=Cc1ccccc1F)CC1(O)C(=O)CCCC1(O)C2=O</smiles>

Brown oil (69 mg, 88\%) obtained from the general procedure (4) using 1c (58 mg, $0.15 \mathrm{mmol}$ ) and 3-acetamidophenylboronic acid (54 $\mathrm{mg}, 0.30 \mathrm{mmol}$ ).

$\mathbf{R}_{\mathbf{f}}=0.29$ (petroleum ether/EtOAc 50:50).

SFC (Chiralpak IF $s \mathrm{CO}_{2} / \mathrm{MeOH} 85: 15,2 \mathrm{~mL} / \mathrm{min}, \mathrm{P}=100$ bar): $\mathrm{Rt}=18.7 \mathrm{~min}$ (minor), 19.8 $\min$ (major).

$[\alpha]_{\mathbf{D}^{20}}=-292\left(\mathrm{c}=1.6, \mathrm{CHCl}_{3}\right)$ for an enantiomeric excess of $91 \%$.

${ }^{1} \mathbf{H}$ NMR $\left(400 \mathrm{MHz}, \mathrm{CDCl}_{3}, \delta\right): 7.92(\mathrm{~d}, J=2.2 \mathrm{~Hz}, 1 \mathrm{H}), 7.63(\mathrm{ddd}, J=15.1,7.6,2.1 \mathrm{~Hz}, 2 \mathrm{H})$, $7.54(\mathrm{~d}, J=8.5 \mathrm{~Hz}, 1 \mathrm{H}), 7.36(\mathrm{~s}, 1 \mathrm{H}), 7.35-7.30(\mathrm{~m}, 2 \mathrm{H}), 7.30-7.27(\mathrm{~m}, 1 \mathrm{H}), 7.18-7.12$ $(\mathrm{m}, 3 \mathrm{H}), 7.03-6.89(\mathrm{~m}, 3 \mathrm{H}), 3.11(\mathrm{~d}, J=13.7 \mathrm{~Hz}, 1 \mathrm{H}), 2.91-2.78(\mathrm{~m}, 2 \mathrm{H}), 2.62-2.42(\mathrm{~m}$, 2H), 2.37 (dd, $J=17.0,1.9 \mathrm{~Hz}, 1 \mathrm{H}), 2.23(\mathrm{~s}, 3 \mathrm{H}), 2.20-2.06(\mathrm{~m}, 2 \mathrm{H}), 1.97$ (d, $J=1.6 \mathrm{~Hz}$, $1 \mathrm{H}), 1.93$ (dt, $J=13.8,4.6 \mathrm{~Hz}, 1 \mathrm{H}), 1.81-1.66(\mathrm{~m}, 1 \mathrm{H})$.

${ }^{13}$ C NMR (101 MHz, $\left.\mathrm{CDCl}_{3}, \delta\right): 212.5,168.5,138.3,137.8,136.1,134.5,133.0,131.7,131.1$ (2C), 130.7, 129.1, $128.7(\mathrm{q}, J=30.3 \mathrm{~Hz}), 128.4(2 \mathrm{C}), 127.2,126.6,126.0,125.9$ (q, $J=5.6$ $\mathrm{Hz}), 124.3$ (q, $J=273.9 \mathrm{~Hz}), 123.6,120.5,114.8,79.0,58.1,37.1,36.5,35.6,33.0,24.8,20.5$. ${ }^{19}$ F NMR (376 MHz, $\left.\mathrm{CDCl}_{3}, \delta\right):-61.0$.

HRMS (ESI): Calculated for $\mathrm{C}_{31} \mathrm{H}_{28} \mathrm{~F}_{3} \mathrm{O}_{3} \mathrm{NNa}(\mathrm{M}+\mathrm{Na})^{+}$542.1919, found 542.1912.

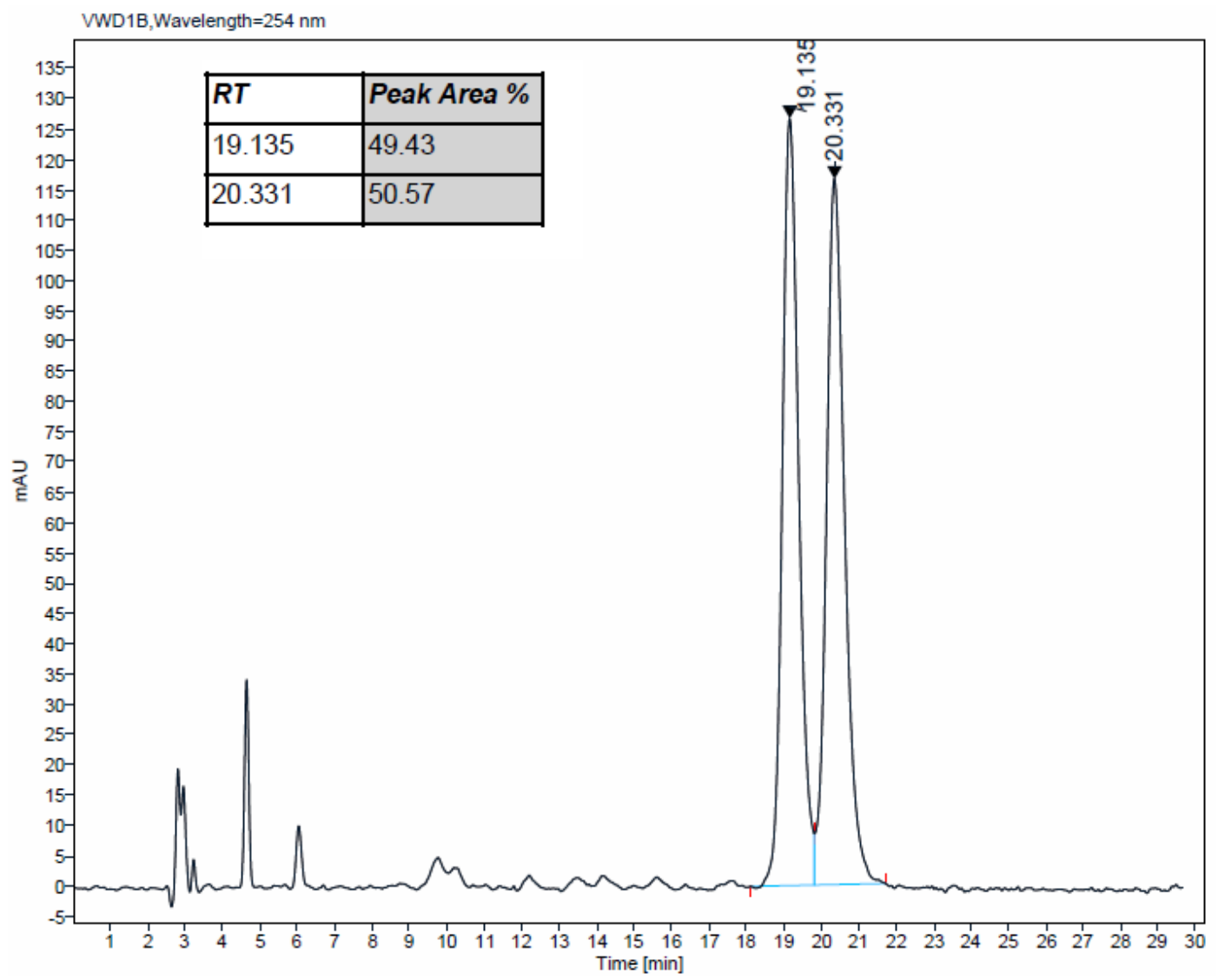




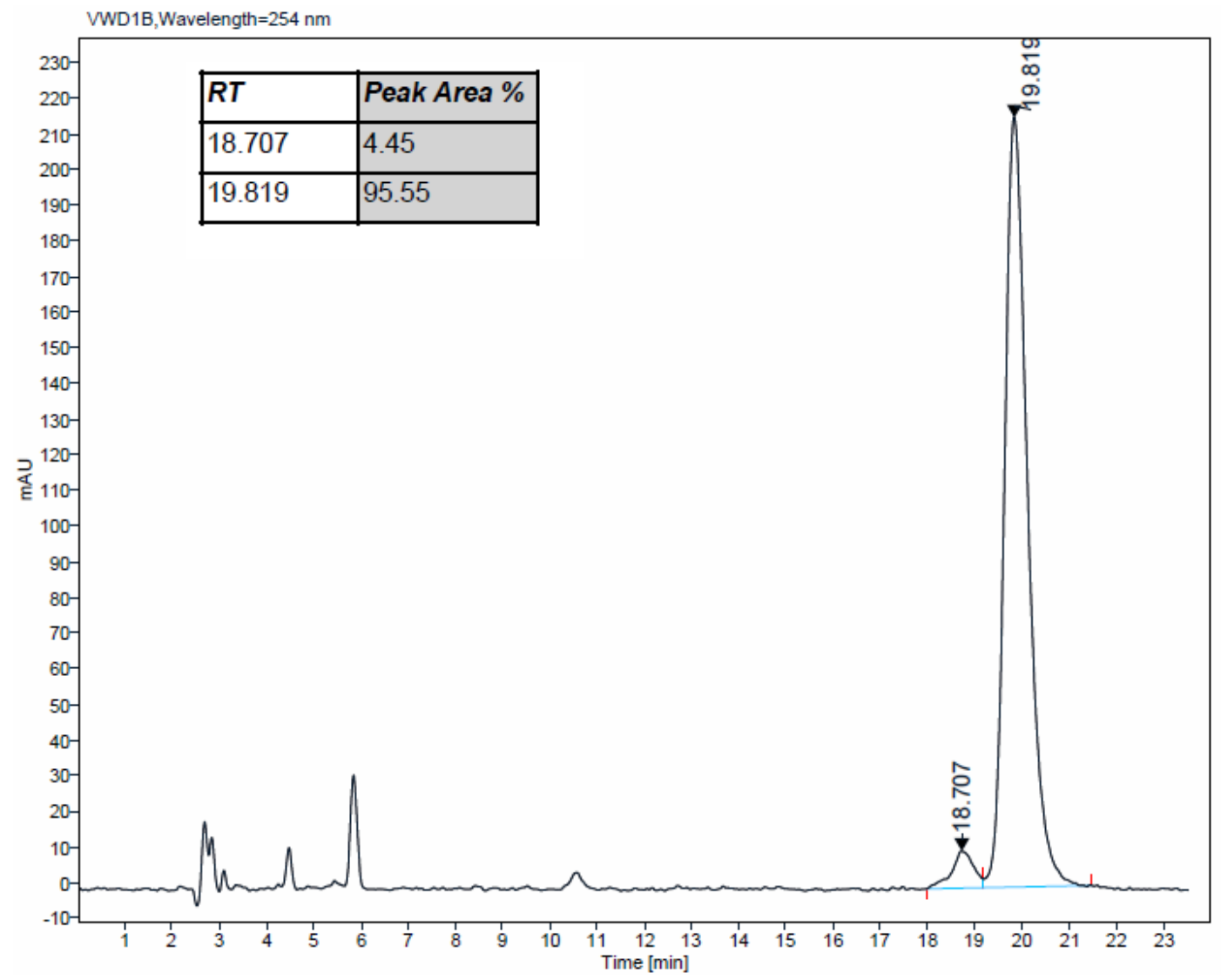

(E)-10a-benzyl-7-fluoro-4a-hydroxy-9-(2-(trifluoromethyl)benzylidene)-3,4,4a,9,10,10ahexahydrophenanthren-1(2H)-one $(2 x)$<smiles>O=C1CCC[C@@]2(O)[C@H](Br)C/C(=C\c3ccccc3F)c3cc(F)ccc3[C@]12O</smiles>

Yellow oil (61 mg, 85\%) obtained from the general procedure (4) using 1c (58 mg, $0.15 \mathrm{mmol})$ and 3-fluorophenylboronic acid (42 $\mathrm{mg}, 0.30 \mathrm{mmol}$ ).

$\mathbf{R}_{\mathbf{f}}=0.22$ (petroleum ether/EtOAc 80:20).

SFC (Chiralpak ADH $s \mathrm{CO}_{2} / \mathrm{MeOH} 90: 10,2 \mathrm{~mL} / \mathrm{min}, \mathrm{P}=100 \mathrm{bar}$ ): $\mathrm{Rt}=13.8 \mathrm{~min}$ (major), 16.5 $\min$ (minor).

$[\alpha]_{\mathbf{D}}{ }^{\mathbf{2 0}}=-243\left(\mathrm{c}=1.0, \mathrm{CHCl}_{3}\right)$ for an enantiomeric excess of $92 \%$.

${ }^{1}$ H NMR $\left(400 \mathrm{MHz}, \mathrm{CDCl}_{3}, \delta\right): 7.68-7.64(\mathrm{~m}, 1 \mathrm{H}), 7.57(\mathrm{dd}, J=8.7,5.9 \mathrm{~Hz}, 1 \mathrm{H}), 7.51(\mathrm{dd}$, $J=10.6,2.6 \mathrm{~Hz}, 1 \mathrm{H}), 7.38-7.32(\mathrm{~m}, 2 \mathrm{H}), 7.31-7.27(\mathrm{~m}, 1 \mathrm{H}), 7.19-7.15(\mathrm{~m}, 3 \mathrm{H}), 7.09$ (td, $J=8.3,2.6 \mathrm{~Hz}, 1 \mathrm{H}), 7.02-6.95(\mathrm{~m}, 2 \mathrm{H}), 6.94-6.89(\mathrm{~m}, 1 \mathrm{H}), 3.09(\mathrm{~d}, J=13.8 \mathrm{~Hz}, 1 \mathrm{H}), 2.86$ $(\mathrm{dd}, J=17.0,2.3 \mathrm{~Hz}, 1 \mathrm{H}), 2.84(\mathrm{~d}, J=13.8 \mathrm{~Hz}, 1 \mathrm{H}), 2.65-2.52(\mathrm{~m}, 1 \mathrm{H}), 2.52-2.43(\mathrm{~m}, 1 \mathrm{H})$, $2.39(\mathrm{dd}, J=17.0,1.5 \mathrm{~Hz}, 1 \mathrm{H}), 2.27-2.08(\mathrm{~m}, 2 \mathrm{H}), 1.95(\mathrm{~d}, J=1.8 \mathrm{~Hz}, 1 \mathrm{H}), 1.94-1.87$ (m, $1 \mathrm{H}), 1.83-1.68(\mathrm{~m}, 1 \mathrm{H})$.

${ }^{13}$ C NMR (101 MHz, $\left.\mathrm{CDCl}_{3}, \delta\right): 212.3,162.5(\mathrm{~d}, J=245.6 \mathrm{~Hz}), 138.1,137.7,135.8,135.7$, 132.5, 131.6, $131.0(2 \mathrm{C}), 130.5,128.6(\mathrm{q}, J=30.1 \mathrm{~Hz}), 128.4(2 \mathrm{C}), 127.3,127.1(\mathrm{~d}, J=8.3$ $\mathrm{Hz}), 126.6,125.9(\mathrm{q}, J=5.5 \mathrm{~Hz}), 124.2(\mathrm{q}, J=273.6 \mathrm{~Hz}), 124.1,115.8(\mathrm{~d}, J=21.6 \mathrm{~Hz}), 110.3$ $(\mathrm{d}, J=22.6 \mathrm{~Hz}), 79.0,57.9,36.9,36.2,35.8,33.1,20.4$.

${ }^{19}$ F NMR $\left(376 \mathrm{MHz}, \mathrm{CDCl}_{3}, \delta\right):-61.2,-114.4$.

HRMS (ESI): Calculated for $\mathrm{C}_{29} \mathrm{H}_{24} \mathrm{~F}_{4} \mathrm{O}_{2} \mathrm{Na}(\mathrm{M}+\mathrm{Na})^{+} 503.1610$, found 503.1602 . 

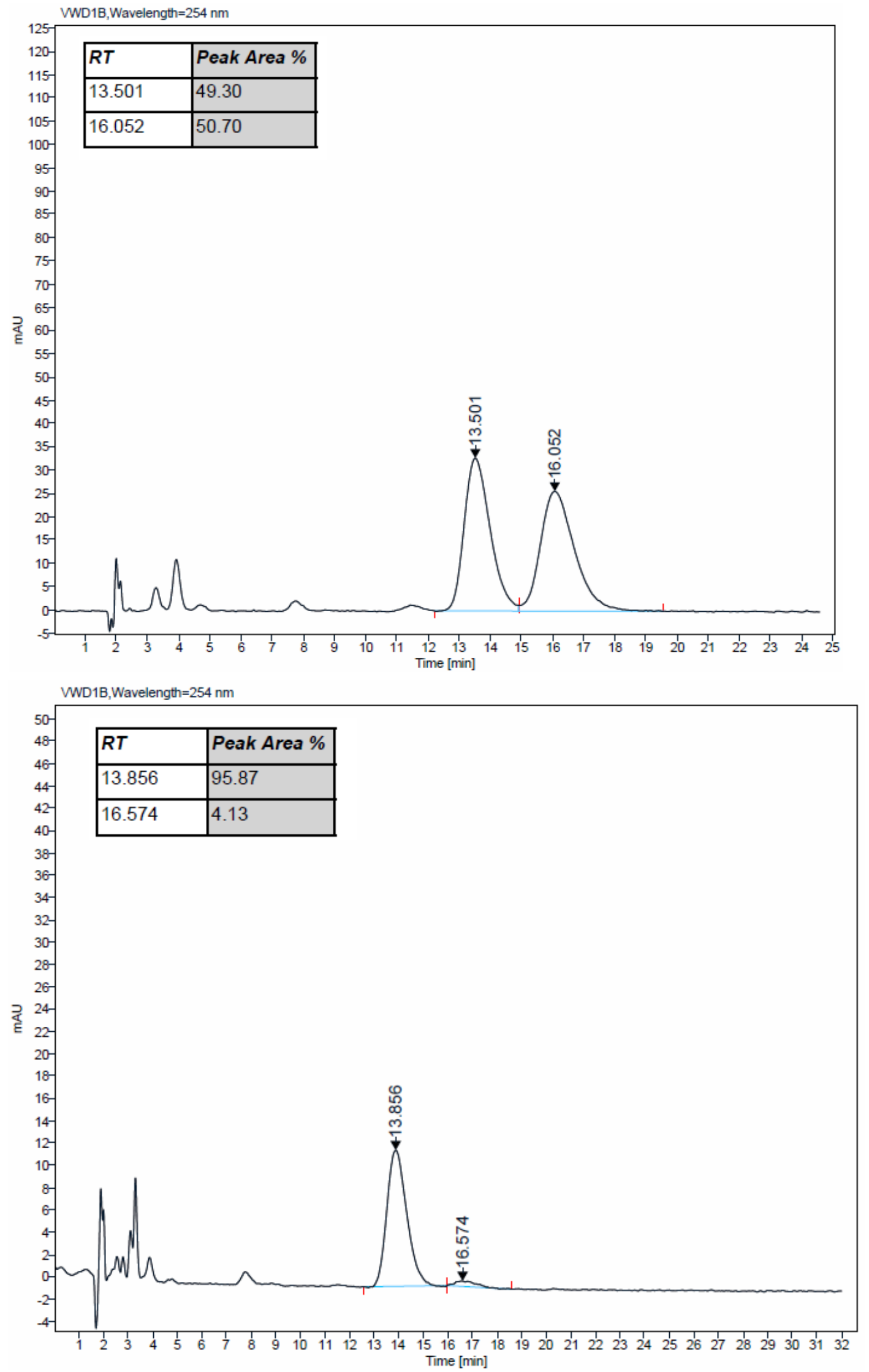

(E)-10a-benzyl-8-fluoro-4a-hydroxy-9-(2-(trifluoromethyl)benzylidene)-3,4,4a,9,10,10ahexahydrophenanthren-1(2H)-one (2y) 


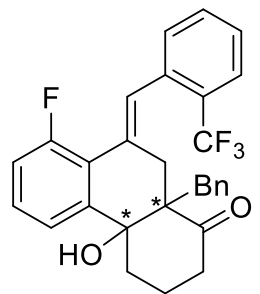

Colorless oil (60 mg, 83\%) obtained from the general procedure (4) using 1c (58 mg, 0.15 $\mathrm{mmol}$ ) and 2-fluorophenylboronic acid (42 $\mathrm{mg}, 0.30 \mathrm{mmol})$.

$\mathbf{R}_{\mathbf{f}}=0.27$ (petroleum ether/EtOAc 80:20).

SFC (Chiralpak ADH $s \mathrm{CO}_{2} / \mathrm{MeOH} 80: 20,2 \mathrm{~mL} / \mathrm{min}, \mathrm{P}=100$ bar): $\mathrm{Rt}=4.1 \mathrm{~min}$ (major), 5.5 $\min$ (minor).

$[\alpha]_{\mathbf{D}}{ }^{20}=-313\left(\mathrm{c}=1.3, \mathrm{CHCl}_{3}\right)$ for an enantiomeric excess of $80 \%$.

${ }^{1} \mathbf{H}$ NMR $\left(400 \mathrm{MHz}, \mathrm{CDCl}_{3}, \delta\right): 7.73-7.63(\mathrm{~m}, 1 \mathrm{H}), 7.59(\mathrm{~s}, 1 \mathrm{H}), 7.47-7.29(\mathrm{~m}, 4 \mathrm{H}), 7.24-$ $7.11(\mathrm{~m}, 4 \mathrm{H}), 7.05-6.93(\mathrm{~m}, 3 \mathrm{H}), 3.02(\mathrm{~d}, J=13.7 \mathrm{~Hz}, 1 \mathrm{H}), 2.78(\mathrm{~d}, J=13.7 \mathrm{~Hz}, 1 \mathrm{H}), 2.76-$ $2.70(\mathrm{~m}, 1 \mathrm{H}), 2.64-2.51(\mathrm{~m}, 1 \mathrm{H}), 2.46-2.36(\mathrm{~m}, 2 \mathrm{H}), 2.18-2.08(\mathrm{~m}, 2 \mathrm{H}), 1.94(\mathrm{~d}, J=1.8$ $\mathrm{Hz}, 1 \mathrm{H}), 1.85-1.75(\mathrm{~m}, 2 \mathrm{H})$.

${ }^{13}$ C NMR (101 MHz, $\left.\mathrm{CDCl}_{3}, \delta\right): 212.5,160.5$ (d, $\left.J=252.4 \mathrm{~Hz}\right), 145.1,138.3,136.4,131.7$, $131.2(2 \mathrm{C}), 130.6,129.9(\mathrm{~d}, J=19.0 \mathrm{~Hz}), 128.9$ (d, $J=9.6 \mathrm{~Hz}), 128.4(2 \mathrm{C}), 128.1$ (q, $J=29.7$ $\mathrm{Hz}), 127.9,127.2,126.7,125.9(\mathrm{q}, J=5.6 \mathrm{~Hz}), 124.1(\mathrm{q}, J=273.7 \mathrm{~Hz}), 122.0(\mathrm{~d}, J=9.6 \mathrm{~Hz})$, $120.2(\mathrm{~d}, J=3.3 \mathrm{~Hz}), 115.8(\mathrm{~d}, J=24.6 \mathrm{~Hz}), 79.6,57.6,36.6,36.4,35.3,34.2,20.3$.

${ }^{19}$ F NMR $\left(376 \mathrm{MHz}, \mathrm{CDCl}_{3}, \delta\right):-61.3,-112.6$.

HRMS (ESI): Calculated for $\mathrm{C}_{29} \mathrm{H}_{24} \mathrm{~F}_{4} \mathrm{O}_{2} \mathrm{Na}(\mathrm{M}+\mathrm{Na})^{+} 503.1610$, found 503.1605 .

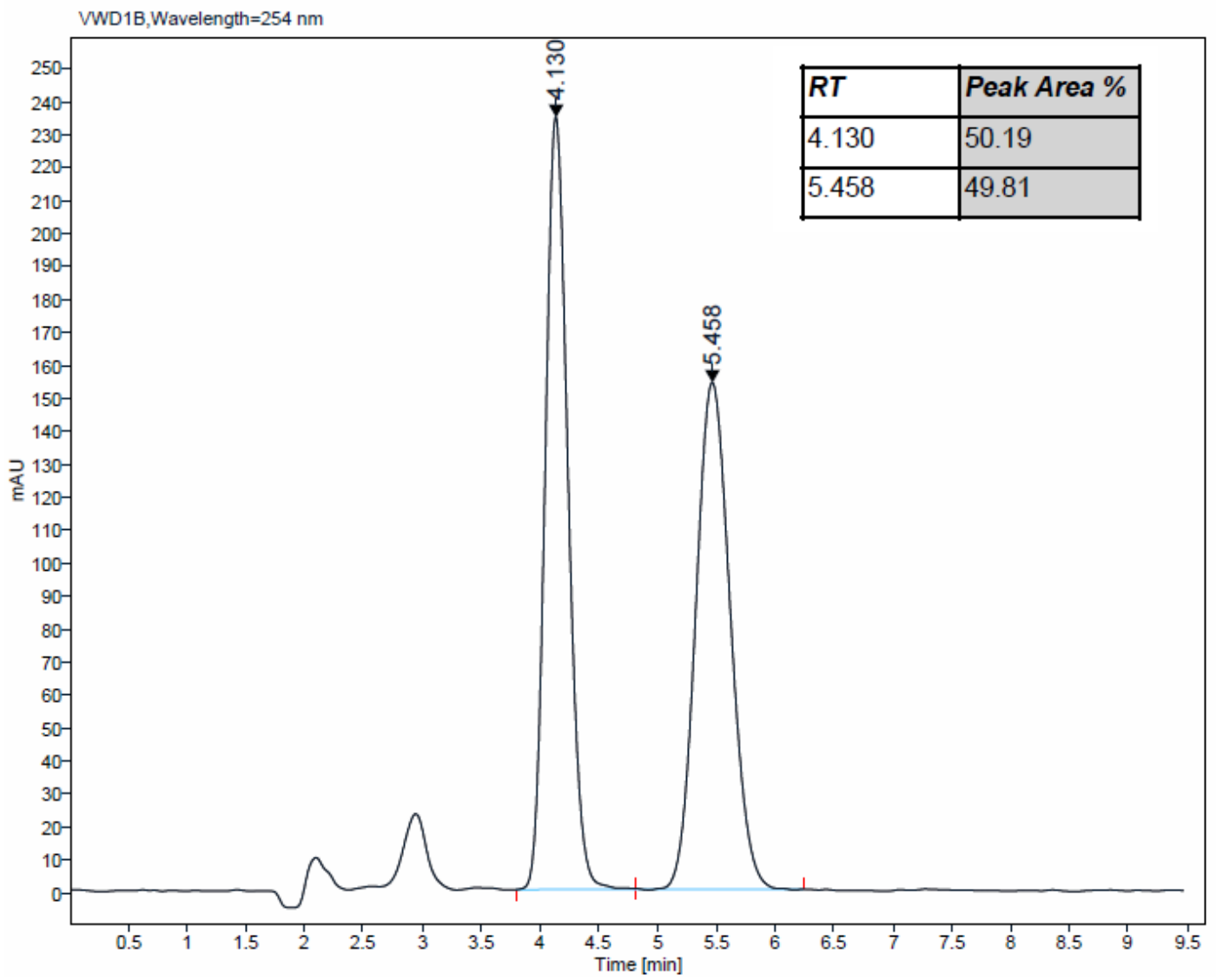




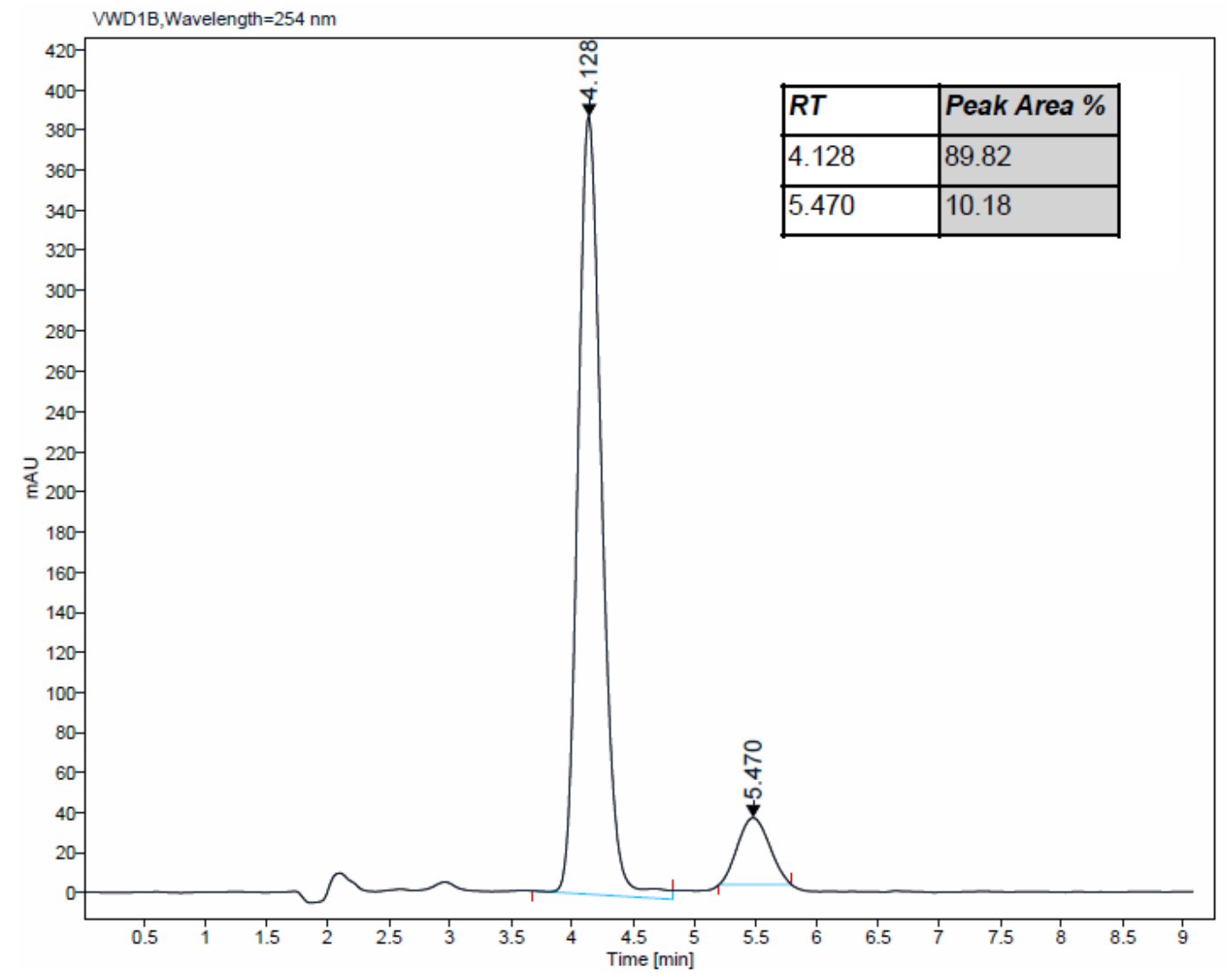

(E)-4a-benzyl-12b-hydroxy-6-(2-(trifluoromethyl)benzylidene)-2,3,4a,5,6,12bhexahydrotetraphen- $4(1 H)-$ one $(2 z)$<smiles>O=C1CCCC2(O)C1(O)C/C(=C/c1ccccc1F)c1cc3ccccc3cc1C2(Br)Br</smiles>

Orange oil (69 mg, 90\%) obtained from the general procedure (4) using 1c (58 $\mathrm{mg}, 0.15 \mathrm{mmol}$ ) and 2-naphthylboronic acid (52 $\mathrm{mg}, 0.30 \mathrm{mmol}$ ).

$\mathbf{R}_{\mathbf{f}}=0.27$ (petroleum ether/EtOAc 80:20).

SFC (Chiralpak ADH $s \mathrm{CO}_{2} / \mathrm{MeOH} 80: 20,2 \mathrm{~mL} / \mathrm{min}, \mathrm{P}=100$ bar): $\mathrm{Rt}=14.2 \mathrm{~min}$ (major), 18.5 $\min$ (minor).

$[\boldsymbol{\alpha}]_{\mathbf{D}}{ }^{\mathbf{2 0}}=-288\left(\mathrm{c}=1.5, \mathrm{CHCl}_{3}\right)$ for an enantiomeric excess of $90 \%$.

${ }^{1} \mathbf{H}$ NMR $\left(400 \mathrm{MHz}, \mathrm{CDCl}_{3}, \delta\right): 8.30(\mathrm{~s}, 1 \mathrm{H}), 8.04(\mathrm{~s}, 1 \mathrm{H}), 8.00-7.91(\mathrm{~m}, 1 \mathrm{H}), 7.90-7.84(\mathrm{~m}$, $1 \mathrm{H}), 7.68(\mathrm{~d}, J=7.6 \mathrm{~Hz}, 1 \mathrm{H}), 7.59-7.48(\mathrm{~m}, 2 \mathrm{H}), 7.45(\mathrm{~s}, 1 \mathrm{H}), 7.42-7.30(\mathrm{~m}, 2 \mathrm{H}), 7.18-$ $7.11(\mathrm{~m}, 3 \mathrm{H}), 7.03-6.96(\mathrm{~m}, 2 \mathrm{H}), 6.92(\mathrm{~d}, J=7.4 \mathrm{~Hz}, 1 \mathrm{H}), 3.12(\mathrm{~d}, J=13.7 \mathrm{~Hz}, 1 \mathrm{H}), 3.00-$ $2.84(\mathrm{~m}, 1 \mathrm{H}), 2.72(\mathrm{~d}, J=13.7 \mathrm{~Hz}, 1 \mathrm{H}), 2.68-2.61(\mathrm{~m}, 1 \mathrm{H}), 2.52(\mathrm{dd}, J=17.6,2.1 \mathrm{~Hz}, 1 \mathrm{H})$, $2.49-2.40(\mathrm{~m}, 1 \mathrm{H}), 2.32-2.22(\mathrm{~m}, 2 \mathrm{H}), 2.08(\mathrm{~d}, J=1.8 \mathrm{~Hz}, 1 \mathrm{H}), 1.98-1.77(\mathrm{~m}, 2 \mathrm{H})$.

${ }^{13}$ C NMR (101 MHz, $\left.\mathrm{CDCl}_{3}, \delta\right): 212.9,140.5,138.5,136.3,133.6,133.3,132.9,132.5,131.8$, $131.3(2 \mathrm{C}), 130.7,128.7$ (q, $J=29.5 \mathrm{~Hz}), 128.3(2 \mathrm{C}), 128.1,127.8,127.2,126.6,126.5,126.5$, $126.0(\mathrm{q}, J=5.1 \mathrm{~Hz}), 124.3(\mathrm{q}, J=273.7 \mathrm{~Hz}), 124.1,123.5,123.4,80.1,57.8,36.8,36.1,35.9$, 33.6, 20.8 .

${ }^{19}$ F NMR $\left(376 \mathrm{MHz}, \mathrm{CDCl}_{3}, \delta\right):-61.3$.

HRMS (ESI): Calculated for $\mathrm{C}_{33} \mathrm{H}_{27} \mathrm{~F}_{3} \mathrm{O}_{2} \mathrm{Na}(\mathrm{M}+\mathrm{Na})^{+}$535.1861, found 535.1855. 

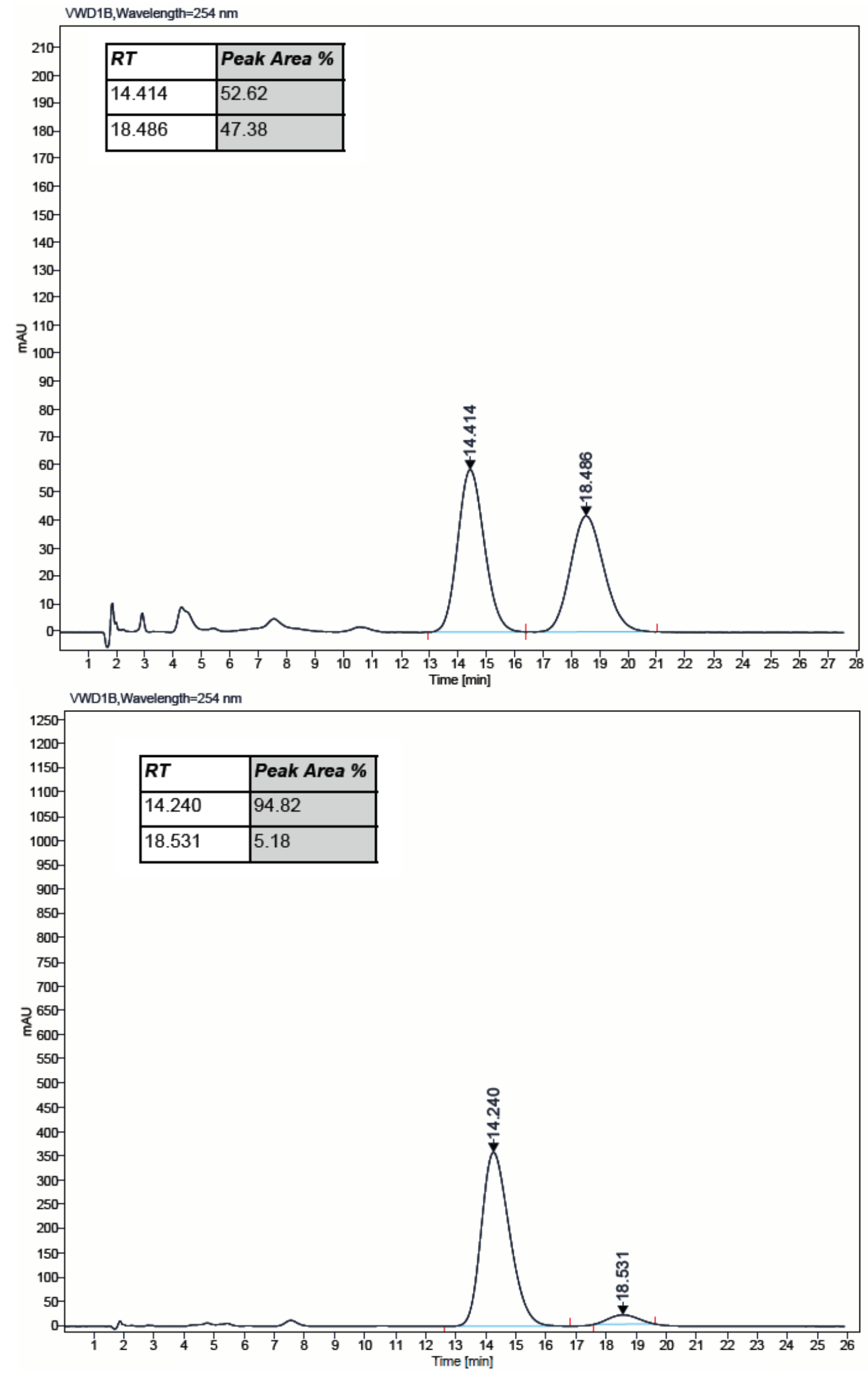

(E)-1-(2-ethyl-1-hydroxy-6-methoxy-1-methyl-4-(2-(trifluoromethyl)benzylidene)1,2,3,4-tetrahydronaphthalen-2-yl)ethan-1-one (2aa) 
<smiles>CCC1(C(C)=O)/C(=C/c2ccccc2F)c2cc(OC)ccc2C1(C)O</smiles>

Yellow oil (54 mg, 86\%) obtained from the general procedure (4) using 1d (47 mg, $0.15 \mathrm{mmol}$ ) and 3-methoxyphenylboronic acid (46 $\mathrm{mg}, 0.30 \mathrm{mmol})$.

$\mathbf{R}_{\mathbf{f}}=0.31$ (petroleum ether/EtOAc 80:20).

SFC (Chiralpak IE $s \mathrm{CO}_{2} / \mathrm{MeOH} 95: 5,2 \mathrm{~mL} / \mathrm{min}, \mathrm{P}=100$ bar): $\mathrm{Rt}=14.7$ min (minor), 15.4 min (major).

$[\alpha]_{\mathbf{D}}{ }^{\mathbf{2 0}}=+57\left(\mathrm{c}=1.9, \mathrm{CHCl}_{3}\right)$ for an enantiomeric excess of $85 \%$.

${ }^{1}$ H NMR $\left(400 \mathrm{MHz}, \mathrm{CDCl}_{3}, \delta\right): 7.84-7.71(\mathrm{~m}, 1 \mathrm{H}), 7.66(\mathrm{~d}, J=8.7 \mathrm{~Hz}, 1 \mathrm{H}), 7.62-7.55(\mathrm{~m}$, 1H), $7.47-7.39(\mathrm{~m}, 2 \mathrm{H}), 7.29-7.27(\mathrm{~m}, 1 \mathrm{H}), 7.15(\mathrm{~d}, J=2.6 \mathrm{~Hz}, 1 \mathrm{H}), 6.90(\mathrm{dd}, J=8.7,2.6$ $\mathrm{Hz}, 1 \mathrm{H}), 4.77(\mathrm{q}, J=1.2 \mathrm{~Hz}, 1 \mathrm{H}), 3.83(\mathrm{~s}, 3 \mathrm{H}), 3.16$ (dd, $J=17.6,1.8 \mathrm{~Hz}, 1 \mathrm{H}), 2.64$ (dd, $J=$ 17.6, $2.7 \mathrm{~Hz}, 1 \mathrm{H}), 2.24-2.10(\mathrm{~m}, 1 \mathrm{H}), 1.90(\mathrm{~s}, 3 \mathrm{H}), 1.58-1.48(\mathrm{~m}, 1 \mathrm{H}), 1.36(\mathrm{~d}, J=1.2 \mathrm{~Hz}$, $3 \mathrm{H}), 0.85(\mathrm{t}, J=7.6 \mathrm{~Hz}, 3 \mathrm{H})$.

${ }^{13} \mathbf{C}$ NMR $\left(101 \mathrm{MHz}, \mathrm{CDCl}_{3}, \delta\right): 218.8,158.8,138.8,136.1,134.7,133.9,131.7,130.8,128.8$ (q, $J=29.4 \mathrm{~Hz}), 127.4,126.34(\mathrm{q}, J=5.3 \mathrm{~Hz}), 126.32,124.2(\mathrm{q}, J=273.6 \mathrm{~Hz}), 122.5,114.7$, 108.7, 75.5, 57.1, 55.3, 34.6, 29.5, 27.7, 26.0, 9.7.

${ }^{19}$ F NMR $\left(376 \mathrm{MHz}, \mathrm{CDCl}_{3}, \delta\right):-61.0$.

HRMS (ESI): Calculated for $\mathrm{C}_{24} \mathrm{H}_{25} \mathrm{~F}_{3} \mathrm{O}_{3} \mathrm{Na}(\mathrm{M}+\mathrm{Na})^{+} 441.1653$, found 441.1642 .

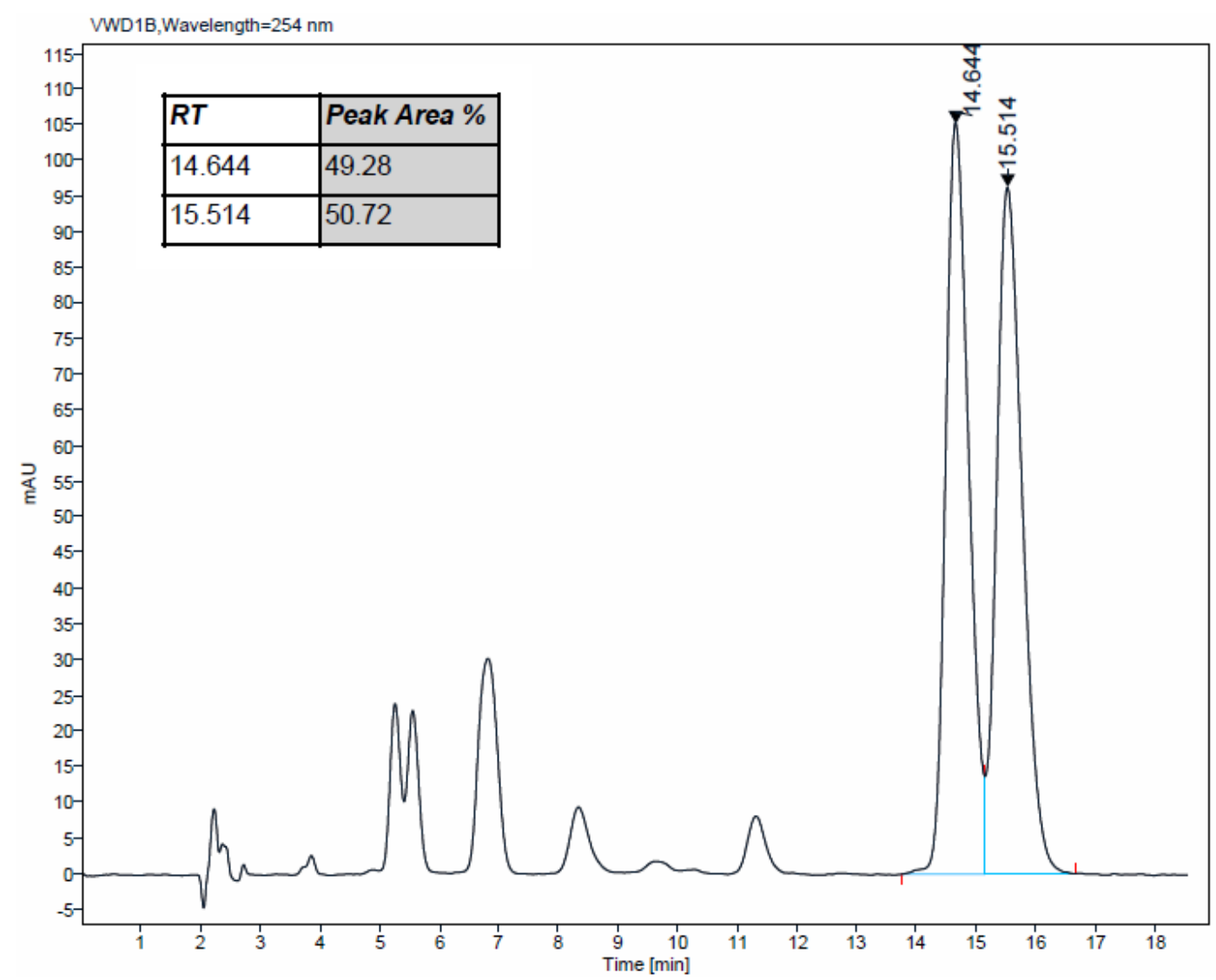




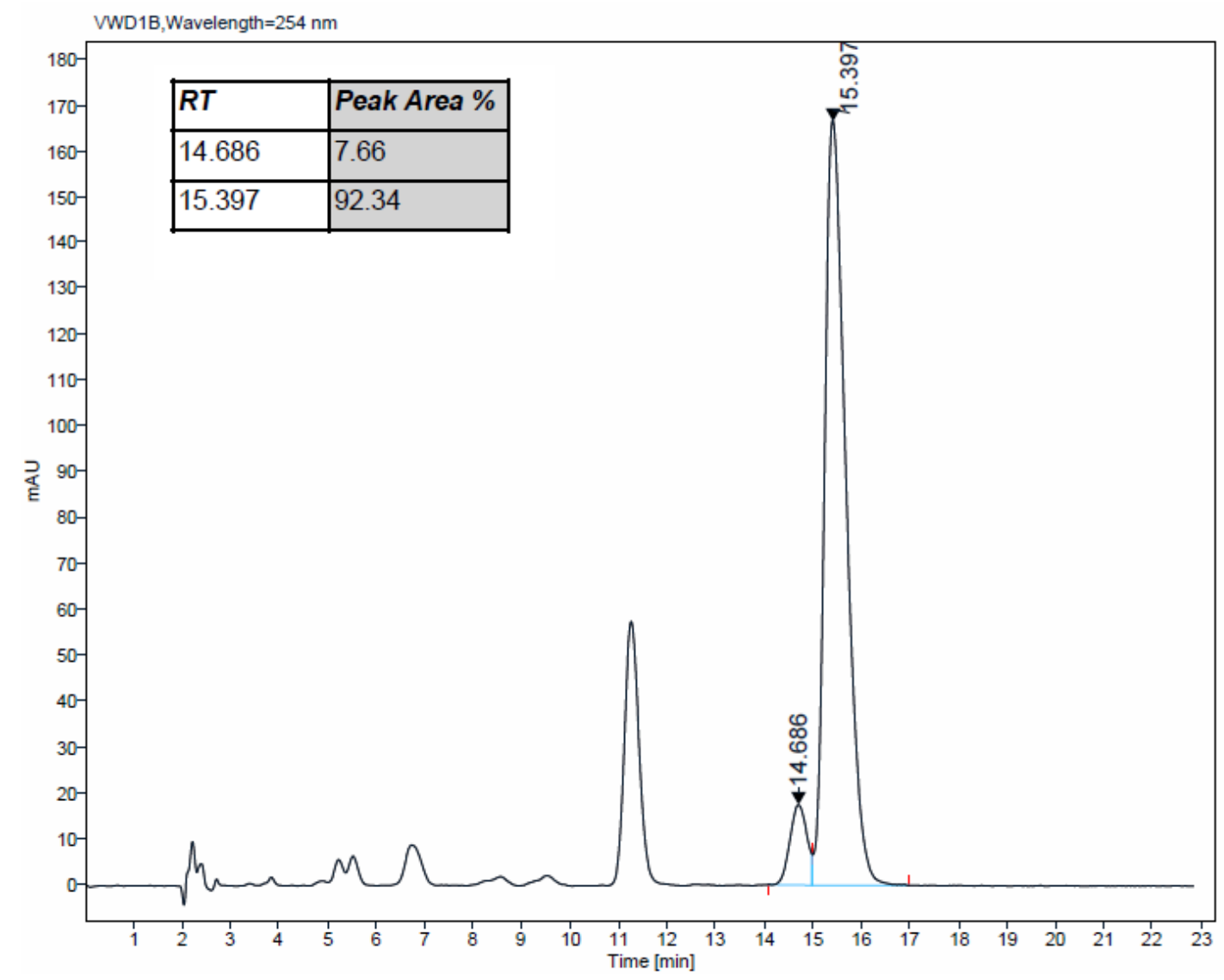

(Z)-12b-hydroxy-5-(2-(trifluoromethyl)benzylidene)-5,12b-dihydroisoindolo[1,2a]isoquinolin-8(6H)-one (3)

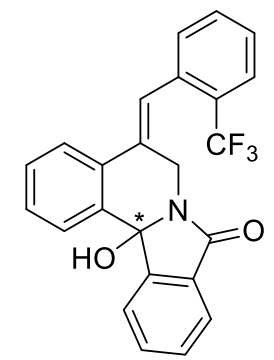

Brown oil (33 mg, 54\%) obtained from the general procedure (4) using 1e (49 mg, $0.15 \mathrm{mmol}$ ) and phenylboronic acid (37 $\mathrm{mg}, 0.30 \mathrm{mmol})$.

$\mathbf{R}_{\mathbf{f}}=0.07$ (petroleum ether/EtOAc 80:20).

SFC (Chiralpak ASH $s \mathrm{CO}_{2} / \mathrm{MeOH} 80: 20,2 \mathrm{~mL} / \mathrm{min}, \mathrm{P}=100$ bar): $\mathrm{Rt}=3.5 \mathrm{~min}$ (major), 10.5 $\min$ (minor).

$[\boldsymbol{\alpha}]_{\mathbf{D}}{ }^{\mathbf{2 0}}=-303\left(\mathrm{c}=0.4, \mathrm{CHCl}_{3}\right)$ for an enantiomeric excess of $81 \%$.

${ }^{1} \mathbf{H}$ NMR $\left(400 \mathrm{MHz}, \mathrm{CDCl}_{3}, \delta\right): 8.02-7.93(\mathrm{~m}, 1 \mathrm{H}), 7.86-7.79(\mathrm{~m}, 1 \mathrm{H}), 7.73(\mathrm{~m}, 1 \mathrm{H}), 7.71-$ $7.65(\mathrm{~m}, 2 \mathrm{H}), 7.63-7.56(\mathrm{~m}, 3 \mathrm{H}), 7.50-7.44(\mathrm{~m}, 1 \mathrm{H}), 7.43-7.37(\mathrm{~m}, 1 \mathrm{H}), 7.37-7.33(\mathrm{~m}$, $2 \mathrm{H}), 7.24(\mathrm{~s}, 1 \mathrm{H}), 5.00(\mathrm{dd}, J=15.4,1.6 \mathrm{~Hz}, 1 \mathrm{H}), 4.03(\mathrm{dd}, J=15.4,1.6 \mathrm{~Hz}, 1 \mathrm{H})$.

${ }^{13}$ C NMR (101 MHz, $\left.\mathrm{CDCl}_{3}, \delta\right): 166.5,146.8,135.3,134.9,134.1,133.1,132.5,132.0,131.0$, $130.9,129.9,129.4,128.6,128.5(\mathrm{q}, J=33.3 \mathrm{~Hz}), 127.7,126.9,126.0(\mathrm{q}, J=4.7 \mathrm{~Hz}), 125.6$, $123.83,123.81(\mathrm{q}, J=272.5 \mathrm{~Hz}), 123.5,123.4,86.6,37.4$.

${ }^{19}$ F NMR $\left(376 \mathrm{MHz}, \mathrm{CDCl}_{3}, \delta\right):-60.7$.

HRMS (ESI): Calculated for $\mathrm{C}_{24} \mathrm{H}_{16} \mathrm{~F}_{3} \mathrm{O}_{2} \mathrm{NNa}(\mathrm{M}+\mathrm{Na})^{+} 430.1031$, found 430.1025. 

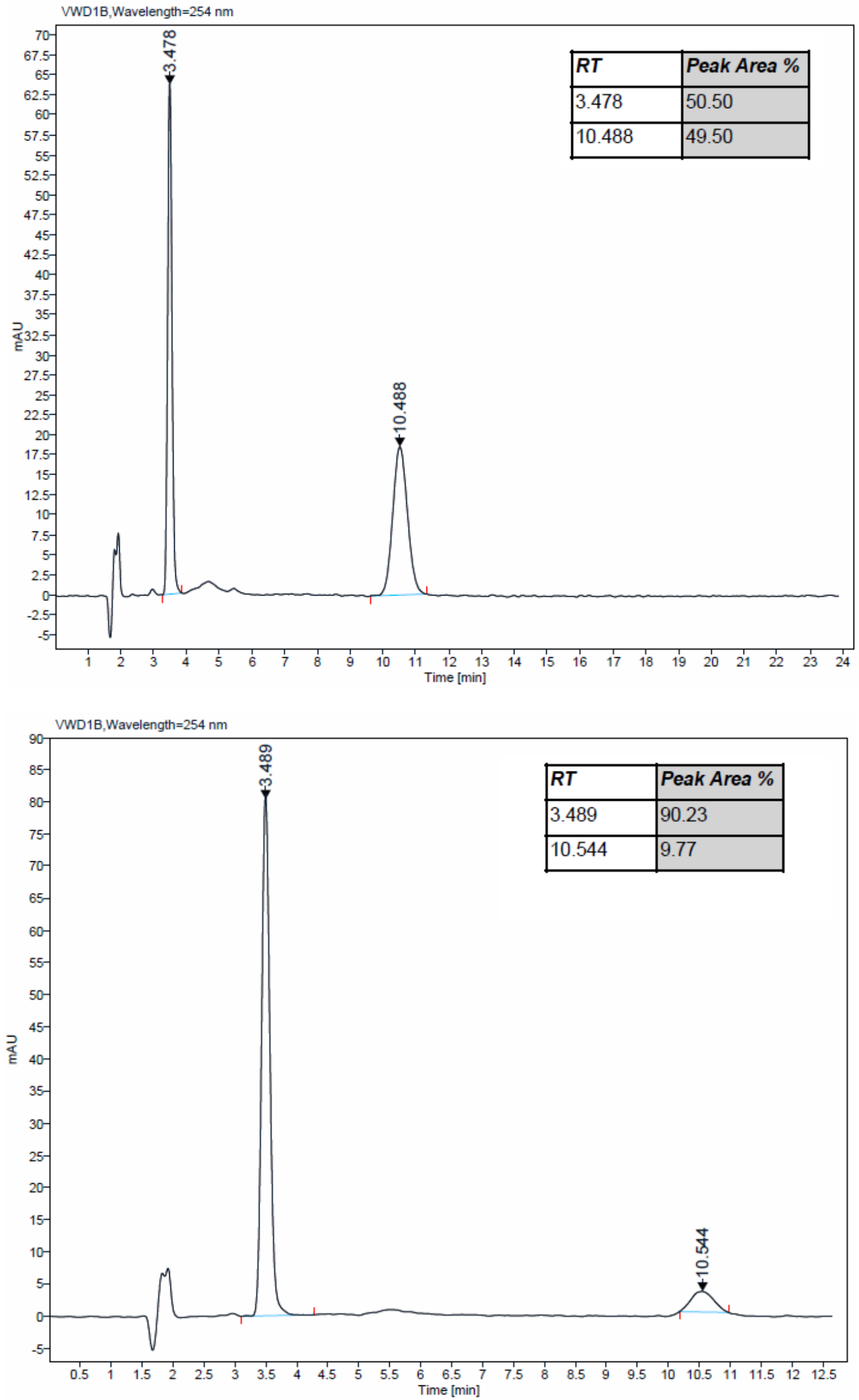


\section{Post-functionalization}

4a-hydroxy-7,10a-dimethyl-2,3,4,4a,10,10a-hexahydrophenanthrene-1,9-dione (4n)<smiles>Cc1ccc2c(c1)C(=Cc1ccccc1F)CC1(O)C(=O)CCCC21O</smiles>

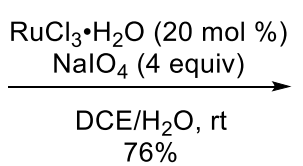<smiles>Cc1ccc2c(c1)C(=O)CC1(C)C(=O)CCCC21O</smiles>

To a suspension of $\mathbf{2 n}(0.10 \mathrm{mmol}, 40 \mathrm{mg})$ and $\mathrm{RuCl}_{3} \cdot 2 \mathrm{H}_{2} \mathrm{O}(20 \mu \mathrm{mol}, 4.9 \mathrm{mg})$ in dichloroethane $(1 \mathrm{~mL})$ and water $(0.75 \mathrm{~mL})$ was added $\mathrm{NaIO}_{4}(0.40 \mathrm{mmol}, 86 \mathrm{mg})$ and the mixture was stirred vigorously. After total consumption of the starting material, monitored by TLC, the reaction was quenched with saturated aqueous solution of $\mathrm{Na}_{2} \mathrm{~S}_{2} \mathrm{O}_{3}(2 \mathrm{~mL}) . \mathrm{CH}_{2} \mathrm{Cl}_{2}$ $(5 \mathrm{~mL})$ was added. The two layers were separated and the aqueous phase was extracted with $\mathrm{CH}_{2} \mathrm{Cl}_{2}$. The combined organic layers were washed with brine, dried over $\mathrm{MgSO}_{4}$, filtered and concentrated under vacuum. The residue was purified by flash chromatography on silica gel (petroleum ether/EtOAc 50:50) to afford $4 \mathbf{n}$ as yellow oil (19 $\mathrm{mg}, 76 \%$ ).

$\mathbf{R}_{\mathbf{f}}=0.26$ (petroleum ether/EtOAc 50:50).

SFC (Chiralpak ADH $s \mathrm{CO}_{2} / \mathrm{MeOH} 80: 20,2.0 \mathrm{~mL} / \mathrm{min}, \mathrm{P}=100 \mathrm{bar}$ ): $\mathrm{Rt}=9.1 \mathrm{~min}$ (major), 10.9 min (minor).

$[\alpha]_{\mathbf{D}}{ }^{\mathbf{2 0}}=+183\left(\mathrm{c}=1.2, \mathrm{CHCl}_{3}\right)$ for an enantiomeric excess of $87 \%$.

${ }^{1}$ H NMR (400 MHz, $\left.\mathrm{CDCl}_{3}, \delta\right): 7.88-7.80(\mathrm{~m}, 1 \mathrm{H}), 7.55(\mathrm{~d}, J=8.0 \mathrm{~Hz}, 1 \mathrm{H}), 7.50-7.43(\mathrm{~m}$, $1 \mathrm{H}), 3.15$ (dd, $J=16.9,0.8 \mathrm{~Hz}, 1 \mathrm{H}), 2.59$ (d, $J=17.0 \mathrm{~Hz}, 1 \mathrm{H}), 2.68-2.47$ (m, 2H), 2.40 (s, $3 \mathrm{H}), 2.41-2.29(\mathrm{~m}, 1 \mathrm{H}), 2.24-2.09(\mathrm{~m}, 2 \mathrm{H}), 2.00(\mathrm{~d}, J=1.6 \mathrm{~Hz}, 1 \mathrm{H}), 1.87-1.74(\mathrm{~m}, 1 \mathrm{H})$, $1.22(\mathrm{~d}, J=0.7 \mathrm{~Hz}, 3 \mathrm{H})$.

${ }^{13} \mathrm{C}$ NMR (101 MHz, $\left.\mathrm{CDCl}_{3}, \delta\right): 211.3,194.8,143.6,138.4,135.7,130.1,127.3,125.3,77.0$, 55.4, 45.6, 36.2, 35.3, 21.0, 20.4, 17.3.

HRMS (ESI): Calculated for $\mathrm{C}_{16} \mathrm{H}_{18} \mathrm{O}_{3} \mathrm{Na}(\mathrm{M}+\mathrm{Na})^{+} 281.1154$, found 281.1147. 

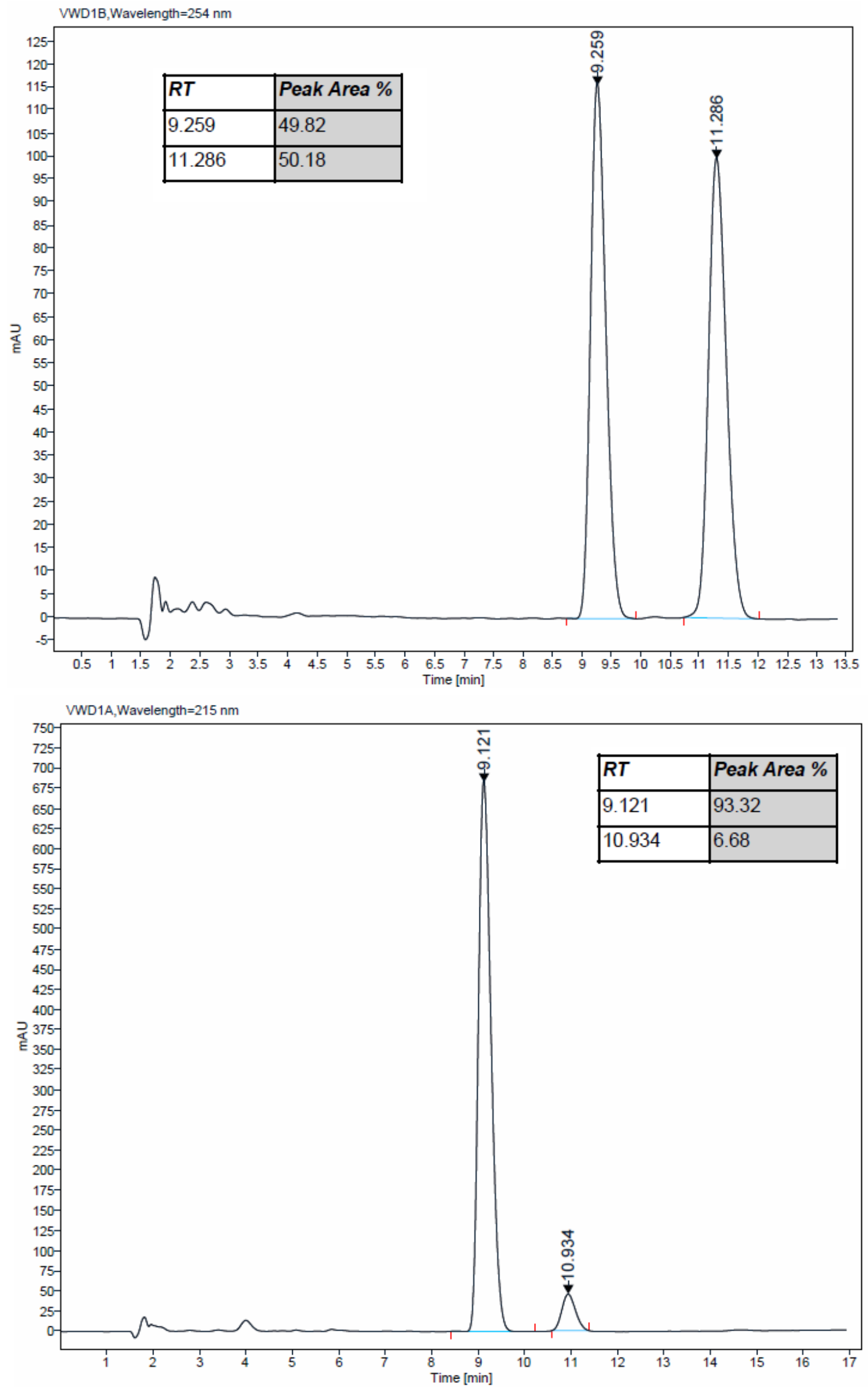

(E)-1-(2-ethyl-1-fluoro-1-methyl-4-(2-(trifluoromethyl)benzylidene)-1,2,3,4tetrahydronaphthalen-2-yl)ethan-1-one (5e) 
<smiles>CC(=O)C1(C)CC(=Cc2ccccc2C(F)(F)F)c2ccccc21</smiles>

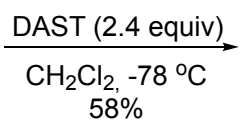

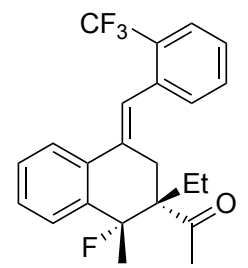

Under argon in a septum-capped vial, equipped with a magnetic stirring bar, was dissolved $\mathbf{2 e}$ $(0.5 \mathrm{mmol}, 194 \mathrm{mg})$ in anhydrous dichloromethane $(5 \mathrm{~mL})$. The resulting solution was cooled to $-78{ }^{\circ} \mathrm{C}$, diethylaminosulfur trifluoride (DAST) $(1.2 \mathrm{mmol}, 0.16 \mathrm{~mL})$ was added and the reaction was then allowed to stir overnight. The reaction was then quenched with water, dichloromethane was added $(15 \mathrm{~mL})$, the aqueous layer was separated, extracted with dichloromethane and the combined organic layers were dried over $\mathrm{MgSO}_{4}$, filtered and concentrated to dryness. The residue was purified by flash chromatography on silica gel (petroleum ether/EtOAc 60:40) to afford 5e as colorless oil (113 mg, $58 \%$ ).

$\mathbf{R}_{\mathbf{f}}=0.68$ (petroleum ether/EtOAc 80:20).

SFC (Chiralpak ADH $s \mathrm{CO}_{2} / \mathrm{MeOH} 95: 5,2.0 \mathrm{~mL} / \mathrm{min}, \mathrm{P}=100$ bar): $\mathrm{Rt}=5.0 \mathrm{~min}$ (major), 6.3 $\min$ (minor).

$[\boldsymbol{\alpha}]_{\mathbf{D}}{ }^{\mathbf{2 0}}=+183\left(\mathrm{c}=1.2, \mathrm{CHCl}_{3}\right)$ for an enantiomeric excess of $80 \%$.

${ }^{1}$ H NMR $\left(400 \mathrm{MHz}, \mathrm{CDCl}_{3}, \delta\right): 7.76-7.67(\mathrm{~m}, 1 \mathrm{H}), 7.65-7.55(\mathrm{~m}, 3 \mathrm{H}), 7.55-7.48(\mathrm{~m}, 1 \mathrm{H})$, $7.46-7.36(\mathrm{~m}, 1 \mathrm{H}), 7.36-7.30(\mathrm{~m}, 2 \mathrm{H}), 7.14(\mathrm{q}, J=2.4 \mathrm{~Hz}, 1 \mathrm{H}), 3.47$ (ddd, $J=16.7,4.7,2.5$ $\mathrm{Hz}, 1 \mathrm{H}), 2.17(\mathrm{dq}, J=13.8,7.5 \mathrm{~Hz}, 1 \mathrm{H}), 2.10-2.01(\mathrm{~m}, 1 \mathrm{H}), 2.03(\mathrm{~d}, J=4.0 \mathrm{~Hz}, 3 \mathrm{H}), 1.56$ $(\mathrm{d}, J=23.0 \mathrm{~Hz}, 3 \mathrm{H}), 1.58-1.48(\mathrm{~m}, 1 \mathrm{H}), 0.64(\mathrm{t}, J=7.5 \mathrm{~Hz}, 3 \mathrm{H})$.

${ }^{13}$ C NMR (101 MHz, $\mathrm{CDCl}_{3}, \delta$ ): 209.3, 138.8 (d, $\left.J=19.6 \mathrm{~Hz}\right), 136.4,135.5,134.9$ (d, $J=4.5$ $\mathrm{Hz}), 131.7,131.1,128.7$ (q, $J=29.3 \mathrm{~Hz}), 128.5,128.2,127.2,126.0$ (q, $J=5.5 \mathrm{~Hz}), 124.2$ (q, $J=274.8 \mathrm{~Hz}), 124.6,123.1,122.9(\mathrm{~d}, J=10.5 \mathrm{~Hz}), 96.4(\mathrm{~d}, J=183.2 \mathrm{~Hz}), 58.7(\mathrm{~d}, J=17.0$ $\mathrm{Hz}), 31.4(\mathrm{~d}, J=5.5 \mathrm{~Hz}), 29.0(\mathrm{~d}, J=7.6 \mathrm{~Hz}), 24.4(\mathrm{~d}, J=2.8 \mathrm{~Hz}), 23.4(\mathrm{~d}, J=29.4 \mathrm{~Hz}), 8.5$. ${ }^{19}$ F NMR $\left(376 \mathrm{MHz}, \mathrm{CDCl}_{3}, \delta\right):-61.3,-151.2$.

HRMS (ESI): Calculated for $\mathrm{C}_{23} \mathrm{H}_{22} \mathrm{OF}_{4} \mathrm{Na}(\mathrm{M}+\mathrm{Na})^{+} 413.1504$, found 413.1503 . 

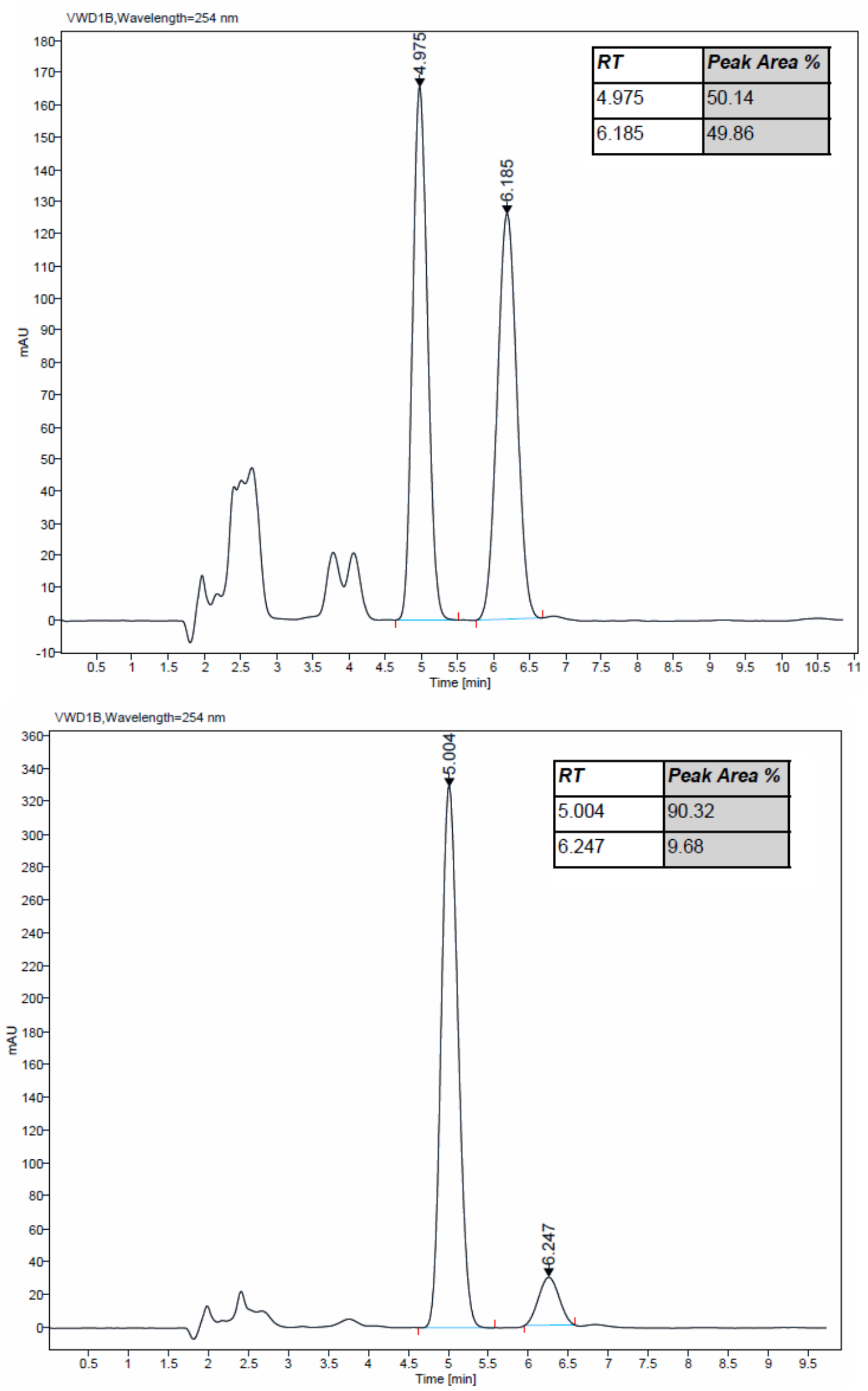


\section{X-ray diffraction of $2 \mathrm{k}$ and $5 \mathrm{e}$}

For compounds $\mathbf{2 k}$ and $\mathbf{5 e}$, a single crystal of this compound was selected, mounted onto a cryoloop and transferred into a cold nitrogen gas stream. Intensity data were collected with a Bruker Kappa APEX-II CCD diffractometer using a micro-focused $\mathrm{Cu}-\mathrm{K} \alpha$ radiation $(\lambda=$ $1.54178 \AA$ ). Data collection was performed at 200K, with the Bruker APEXIII suite. Unit-cell parameters determination, integration and data reduction were carried out with SAINT program. SADABS was used for scaling and absorption corrections. The structure was solved with SHELXT ${ }^{1}$ and refined by full-matrix least-squares methods with SHELXL ${ }^{2}$ using Olex2 software package ${ }^{3}$. All non-hydrogen atoms were refined anisotropically. These structures were deposited at the Cambridge Crystallographic Data Centre (CCDC 1939901 for $\mathbf{2 k}$ and CCDC 1987761 for 5e) and can be obtained free of charge via www.ccdc.cam.ac.uk.

Crystal data for 2 k. colorless needles: $\mathrm{C}_{24} \mathrm{H}_{23} \mathrm{~F}_{3} \mathrm{O}_{3}$, Monoclinic, $P 2{ }_{1}{ }_{2}{ }_{1}{ }_{1}$, a $=7.62580(10), \mathrm{b}=$ 7.92830(10), c = 16.9327(3) $\AA, V=1022.74(3) \AA^{3}, Z=2, T=200(2) K, \mu=0.895 \mathrm{~mm}^{-1}, 27401$ reflections measured, 3608 independent $\left(\mathrm{R}_{\text {int }}=0.0330\right)$, 3499cobserved [I $\geq 2 \sigma$ (I)], 277 parameters, final $\mathrm{R}$ indices $\mathrm{R}_{1}[\mathrm{I} \geq 2 \sigma(\mathrm{I})]=0.0271$ and $\mathrm{wR}_{2}$ (all data) $=0.0678$, GOF on $\mathrm{F}^{2}=$ $1.033, \mathrm{max} / \mathrm{min}$ residual electron density $=0.20 /-0.17 \mathrm{e} . \AA^{-3}$.

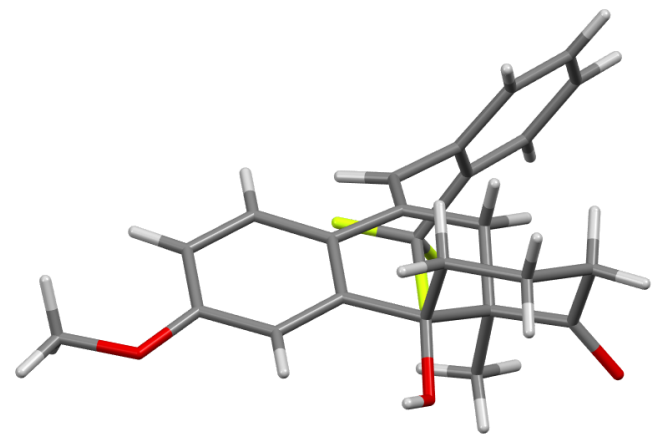

Crystal data for 5e. colorless needles: $\mathrm{C}_{23} \mathrm{H}_{22} \mathrm{~F}_{4} \mathrm{O}$, Orthorhombic, $P 2_{1} 2_{1} 2_{1}$, a $=7.9955(3), \mathrm{b}=$ 11.5397(4), $c=20.8475(7) \AA, V=1923.51(12) \AA^{3}, Z=4, T=200(2) \mathrm{K}, \mu=0.910 \mathrm{~mm}^{-1}, 26483$ reflections measured, 3518 independent $\left(\mathrm{R}_{\text {int }}=0.0361\right), 3416$ observed $[\mathrm{I} \geq 2 \sigma$ (I)], 256 parameters, final $\mathrm{R}$ indices $\mathrm{R}_{1}[\mathrm{I} \geq 2 \sigma(\mathrm{I})]=0.0265$ and $\mathrm{wR}_{2}$ (all data) $=0.0668$, GOF on $\mathrm{F}^{2}=$ $1.049, \mathrm{max} / \mathrm{min}$ residual electron density $=0.15 /-0.14 \mathrm{e} . \AA^{-3}$.

\footnotetext{
${ }^{1}$ Palatinus, L.; Chapuis, G. Journal of Applied Crystallography 2007, 40, 786-790.

${ }^{2}$ Sheldrick, G. M. Acta Crystallographica Section C 2015, 71, 3-8.

${ }^{3}$ Dolomanov, O.V.; Bourhis, L. J.; Gildea, R. J.; Howard, J. A. K.; Puschmann, H. Journal of Applied Crystallography 2009, 42, 339-341.
} 


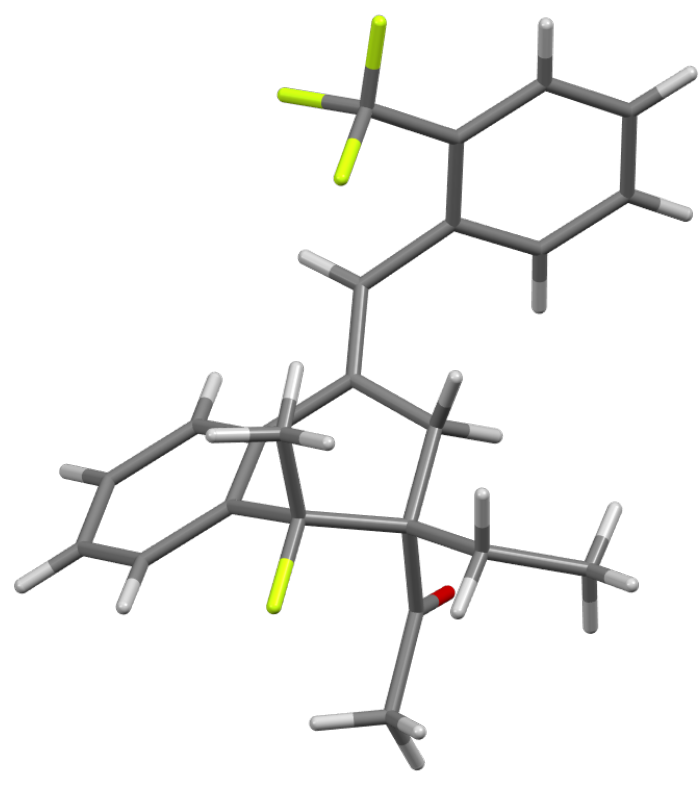




\section{NMR spectra}

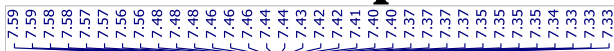

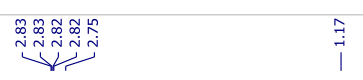

$\mathrm{CDCl} 3,400 \mathrm{MHz}$

$1 /$
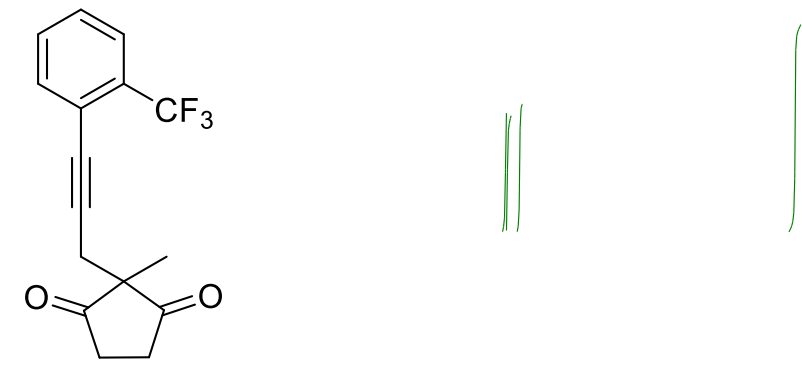

1a

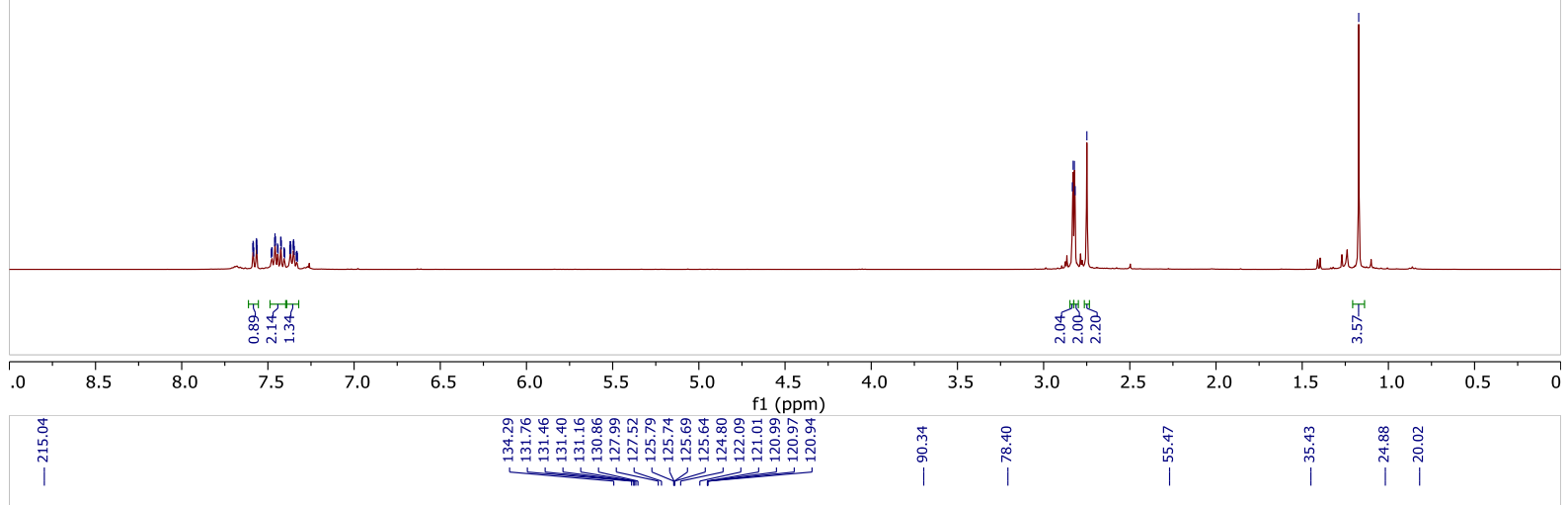

$\mathrm{CDCl} 3,101 \mathrm{MHz}$

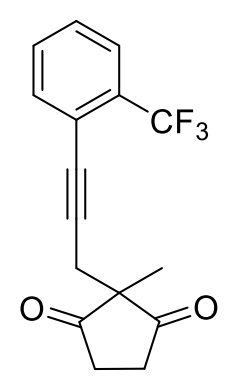

$1 a$

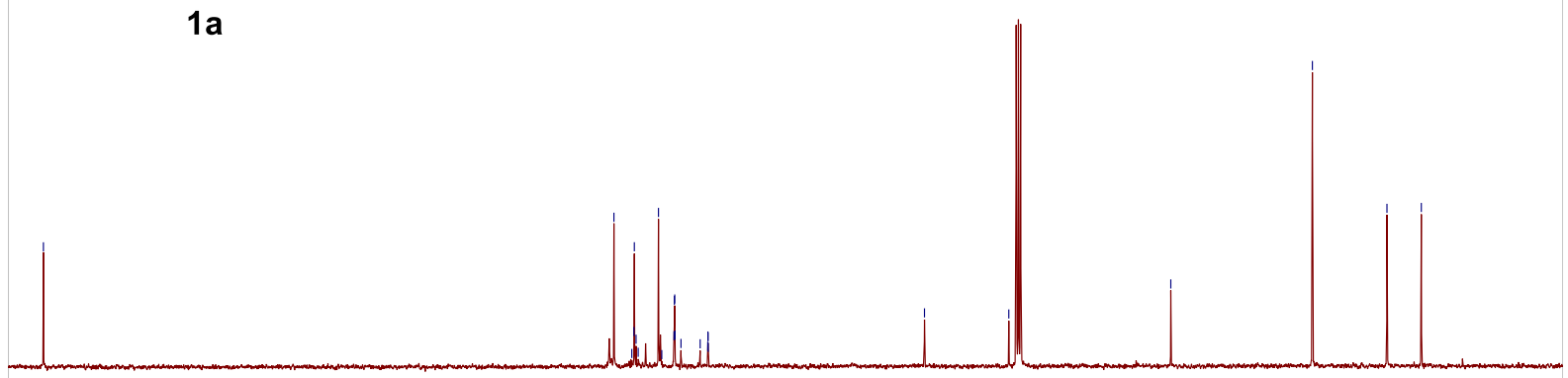

20

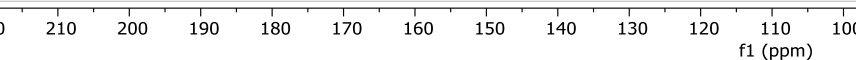




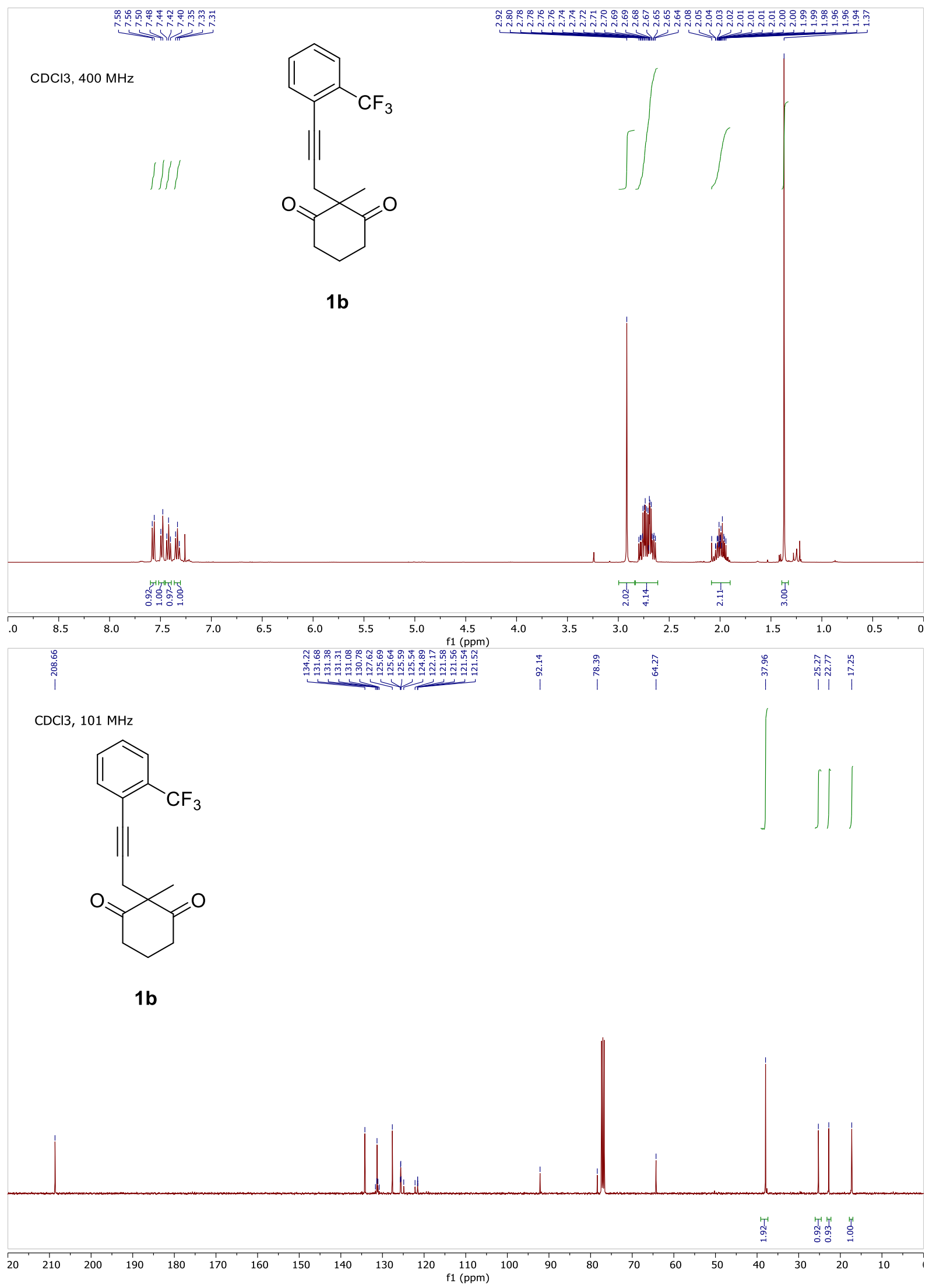

S56 
$\mathrm{CDCl} 3,400 \mathrm{MHz}$
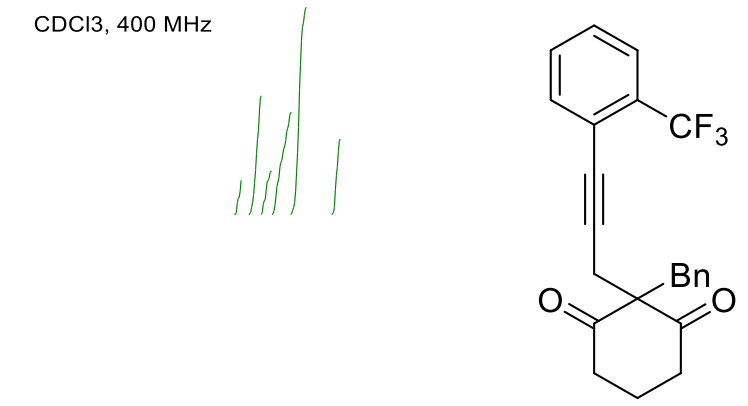

$1 c$

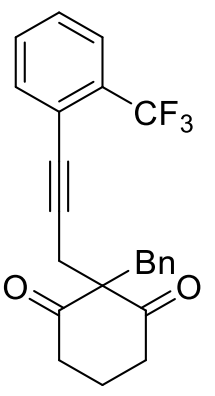

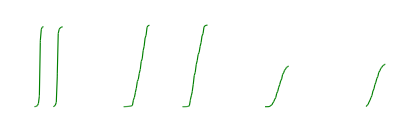

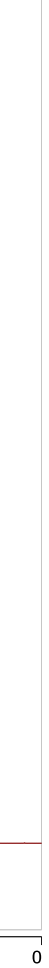

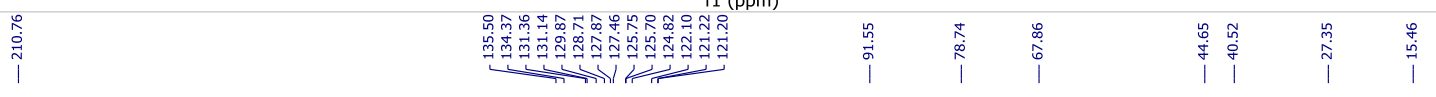

$\mathrm{CDCl} 3,101 \mathrm{MHz}$<smiles>O=C1CCCC(=O)C1(CBr)CC#Cc1ccccc1C(F)(F)F</smiles>

$1 \mathrm{c}$
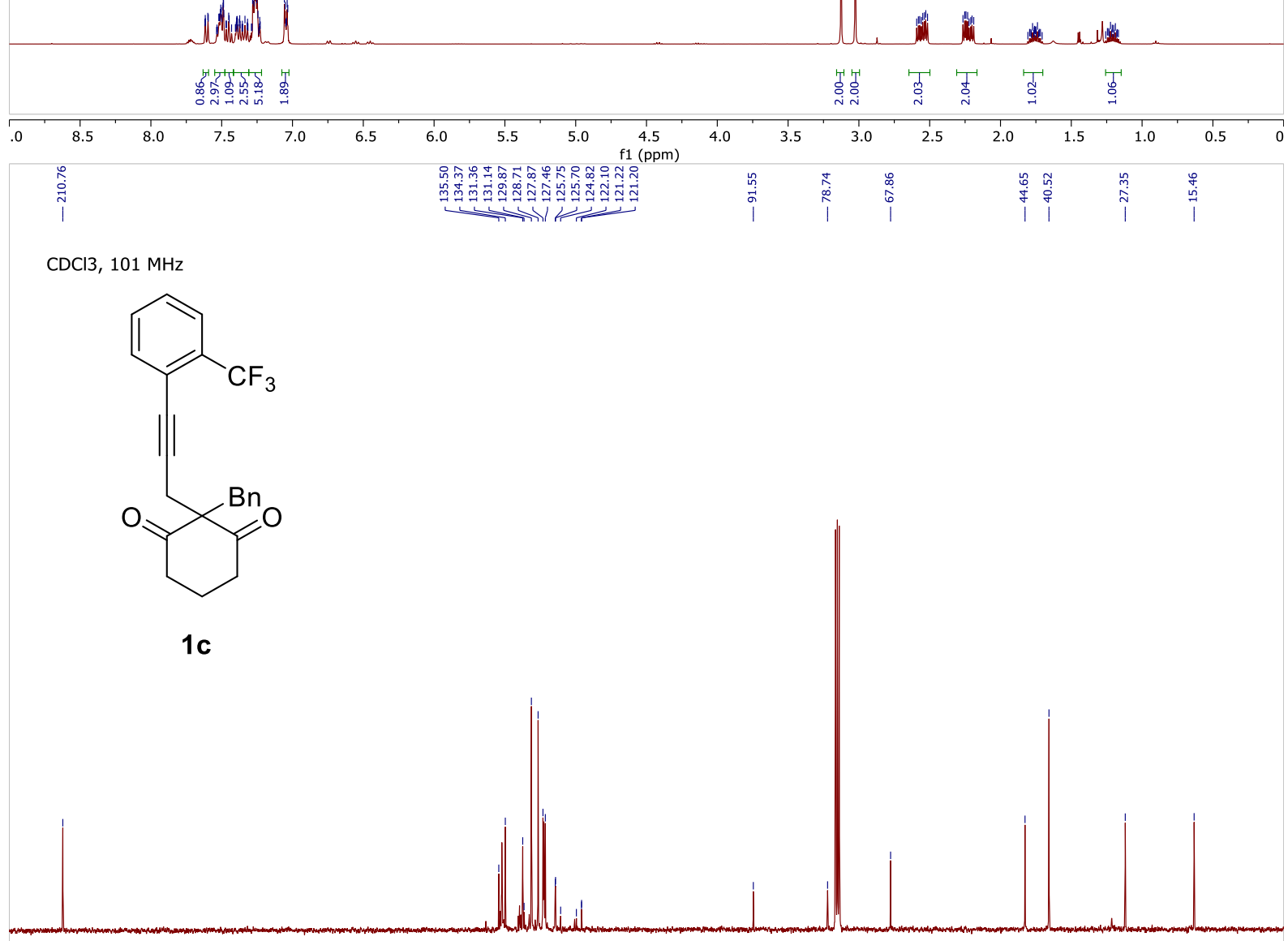

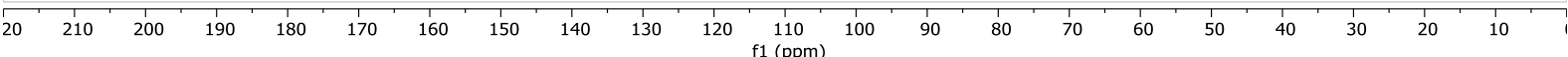




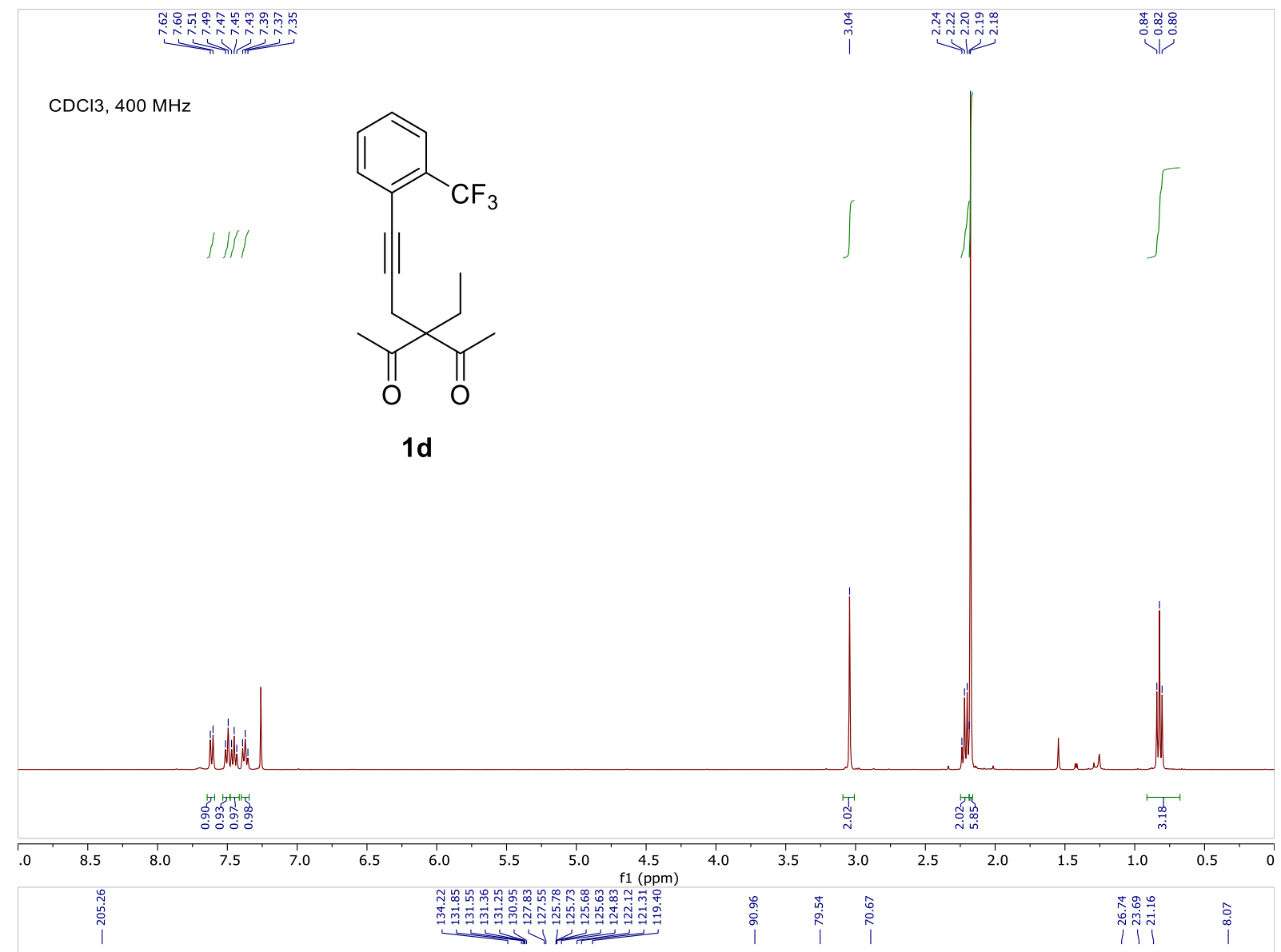

$\mathrm{CDCl} 3,101 \mathrm{MHz}$<smiles>CCC(CC)C(C)=O</smiles>

1d

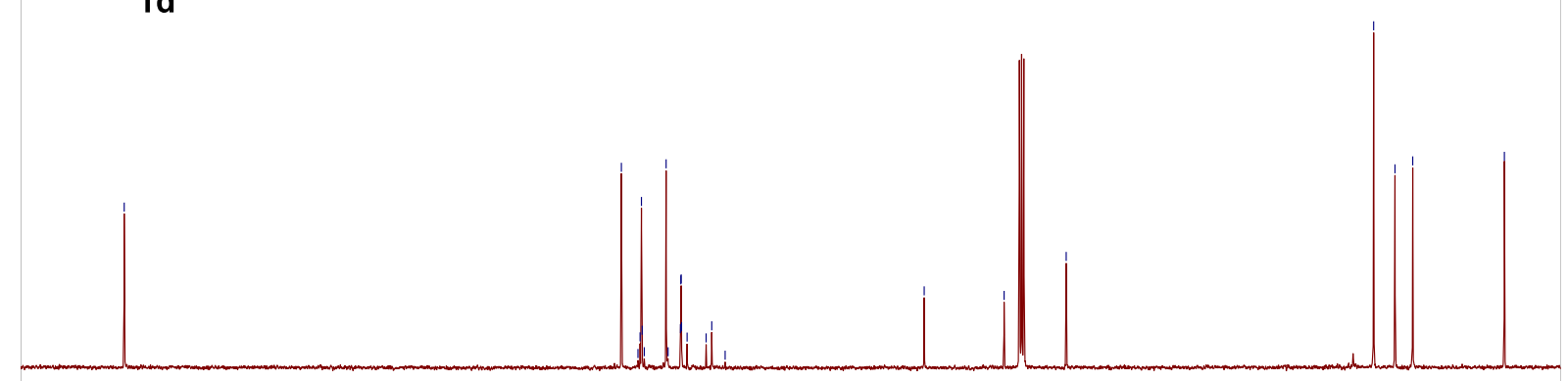

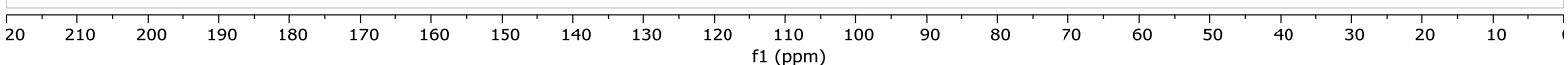




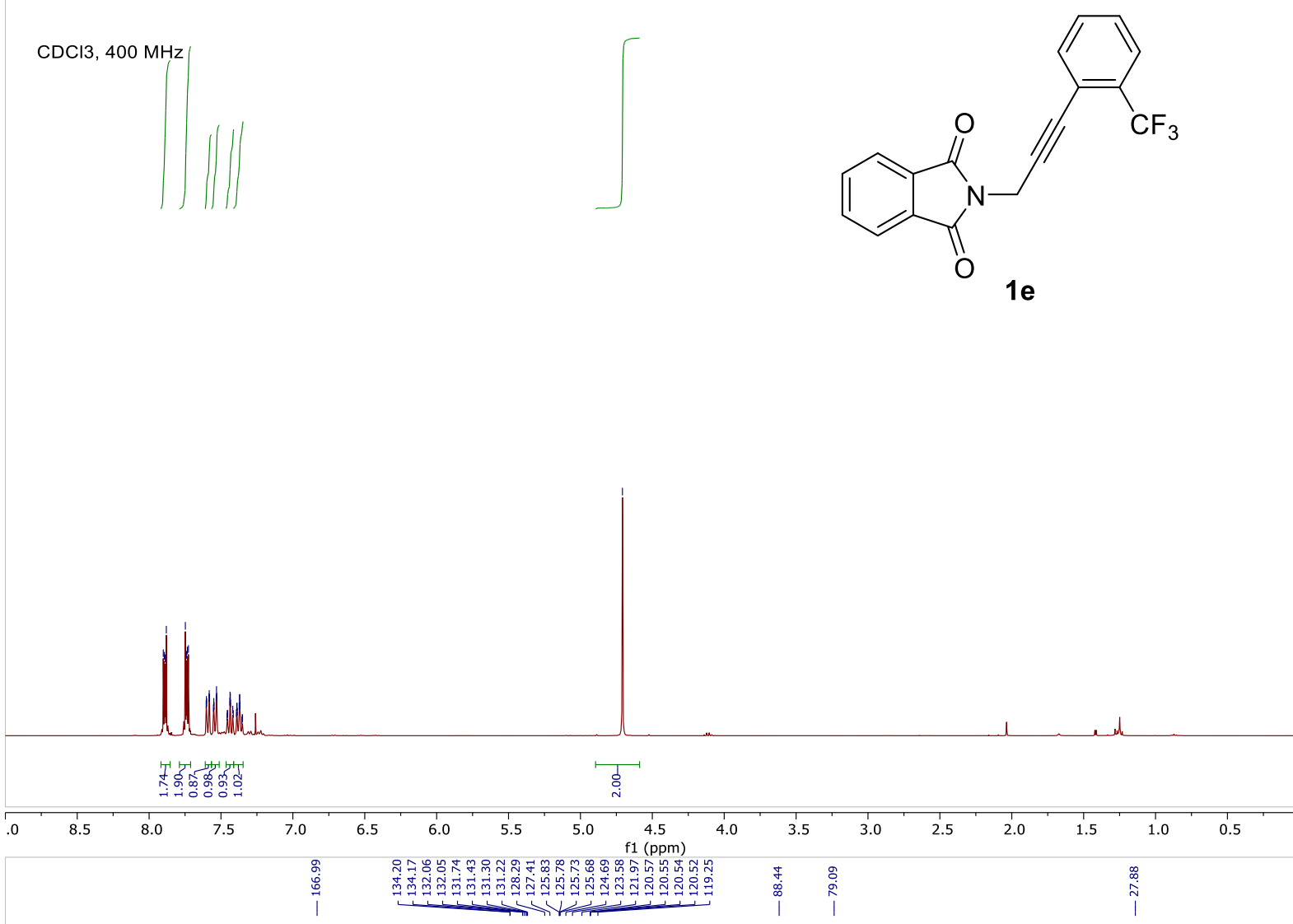

CDCl3, $101 \mathrm{MHz}$

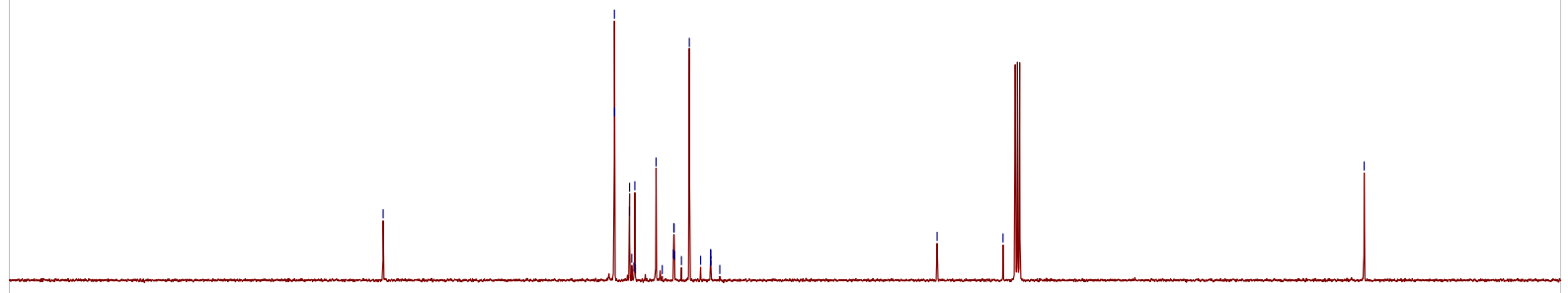

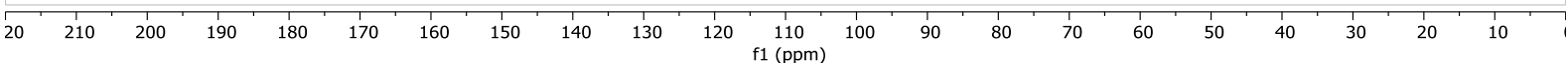




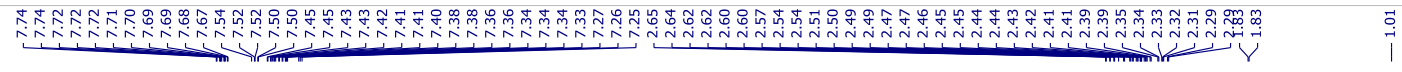

$\mathrm{CDCl} 3,400 \mathrm{MHz}$
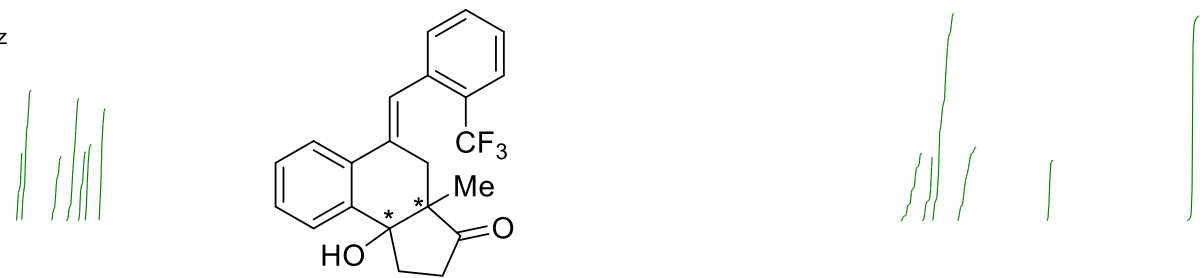

$2 a$

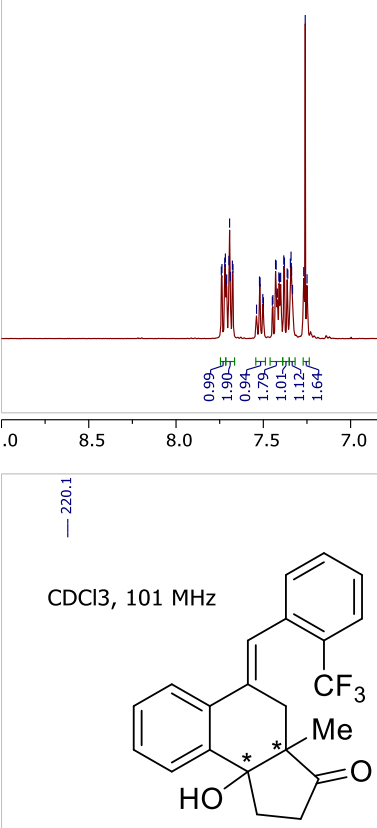

$2 \mathbf{a}$

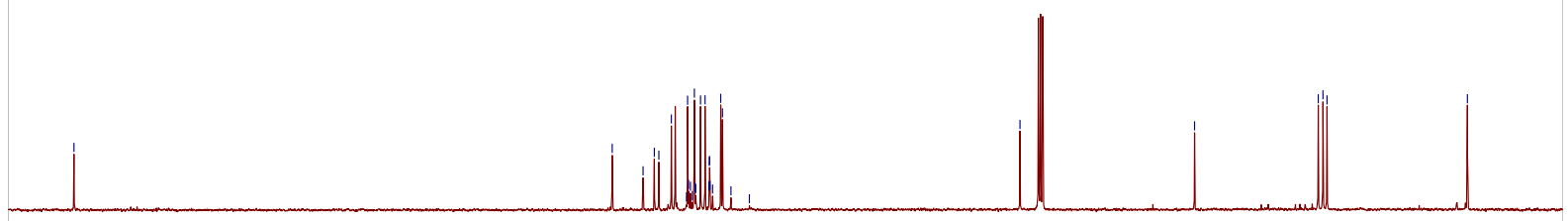

$\begin{array}{lllllllllllllllllllllll}1 & 1 \\ 220 & 210 & 200 & 190 & 180 & 170 & 160 & 150 & 140 & 130 & 120 & 110 & 100 & 90 & 80 & 70 & 60 & 50 & 40 & 30 & 20 & 10 & 1\end{array}$ 


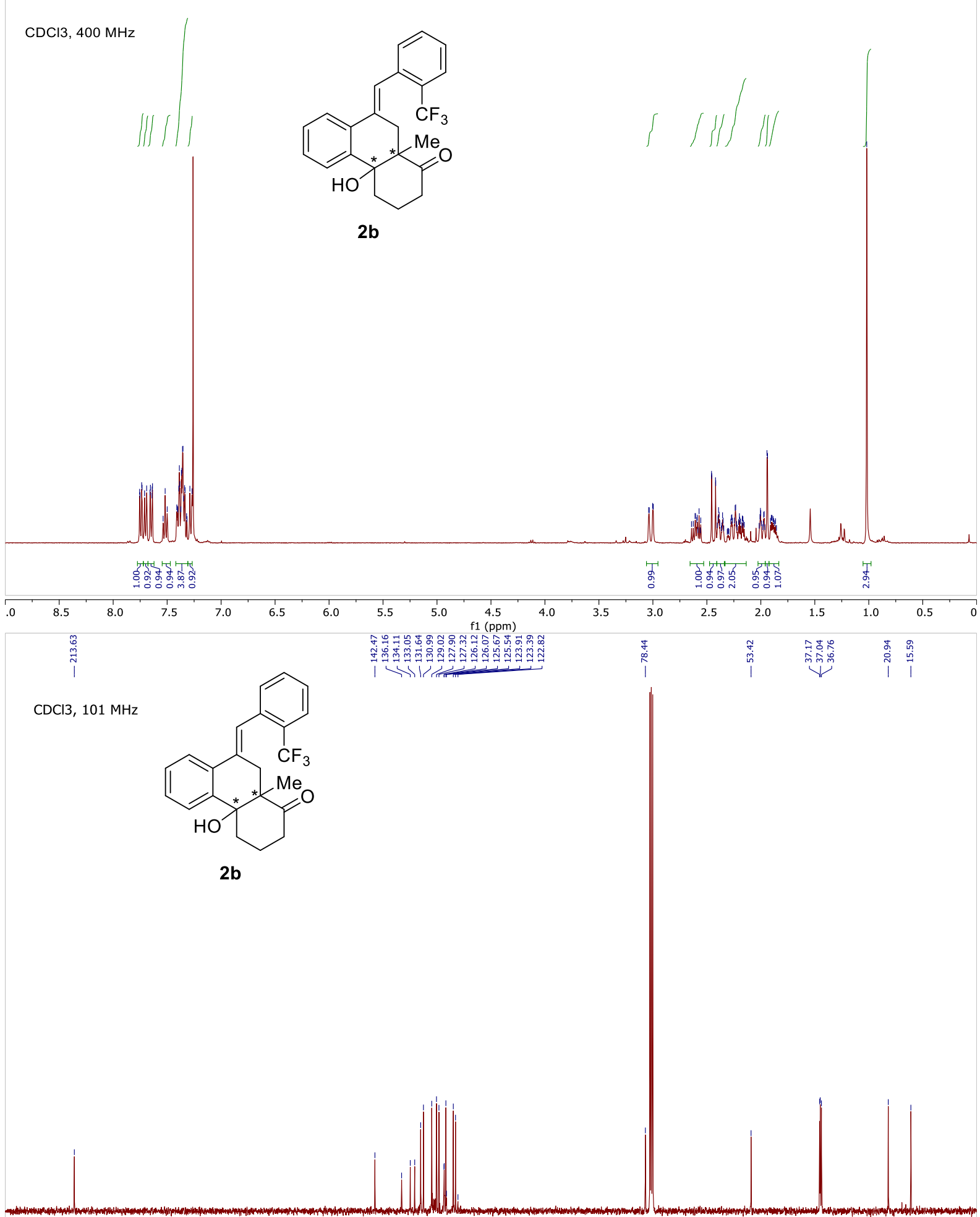

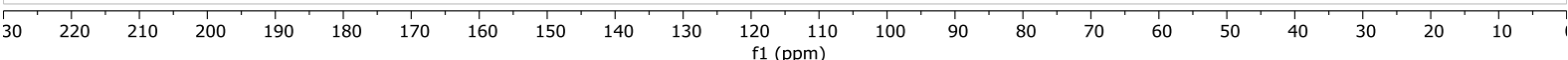




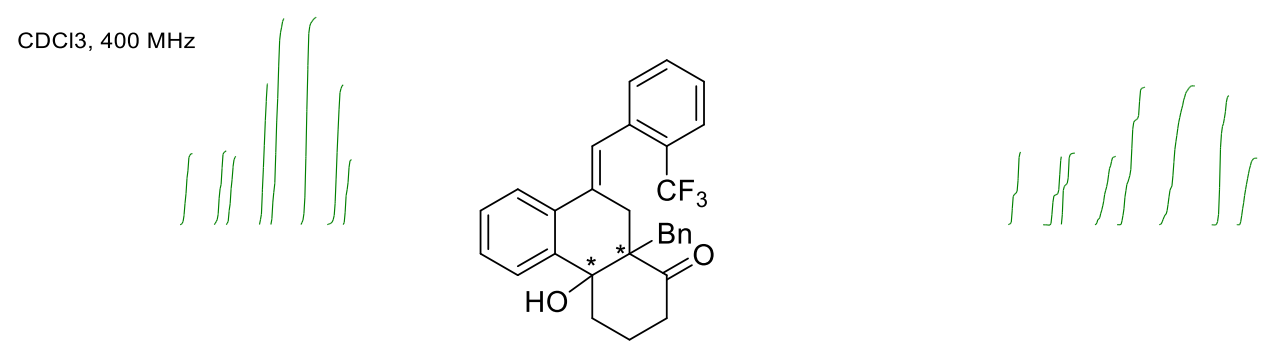

2c

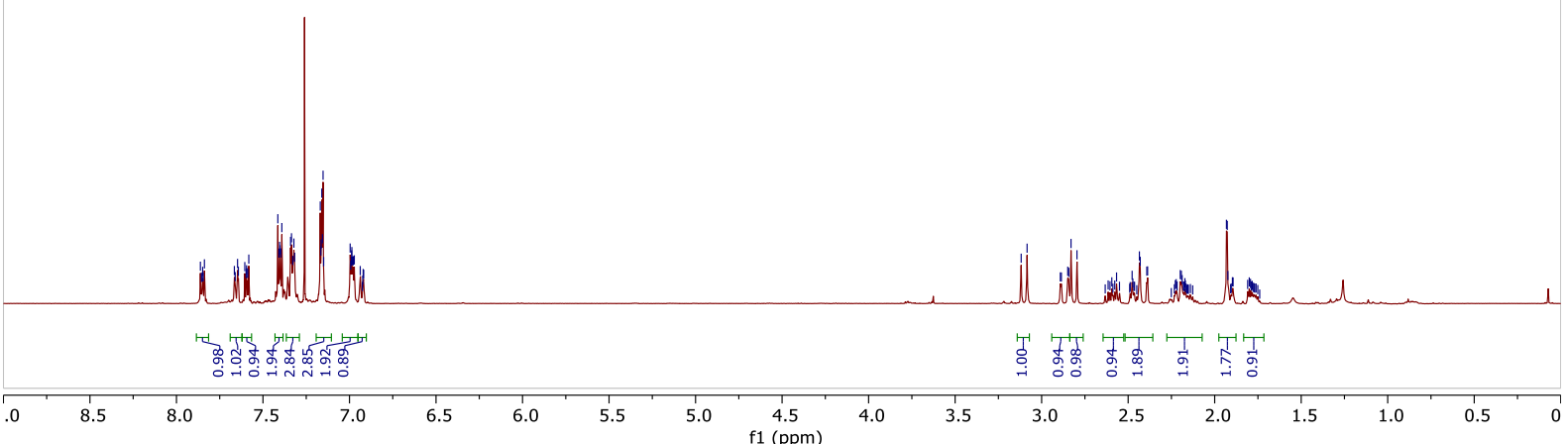

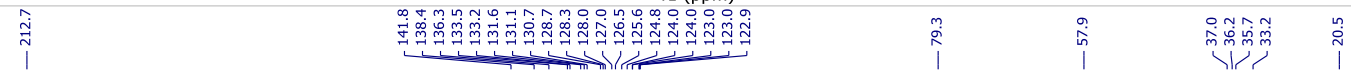

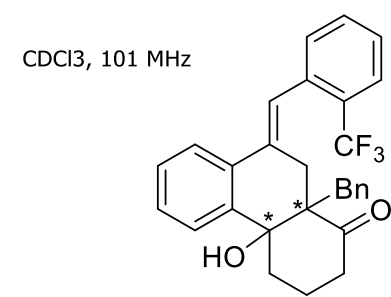

2c

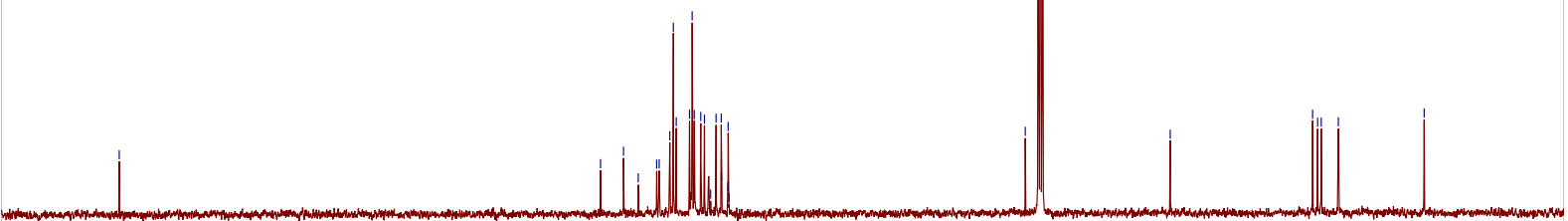

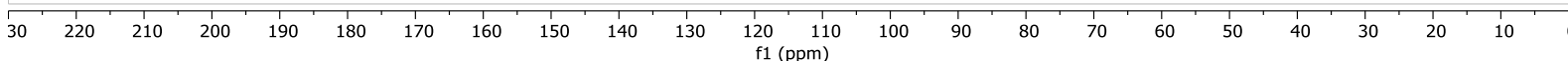




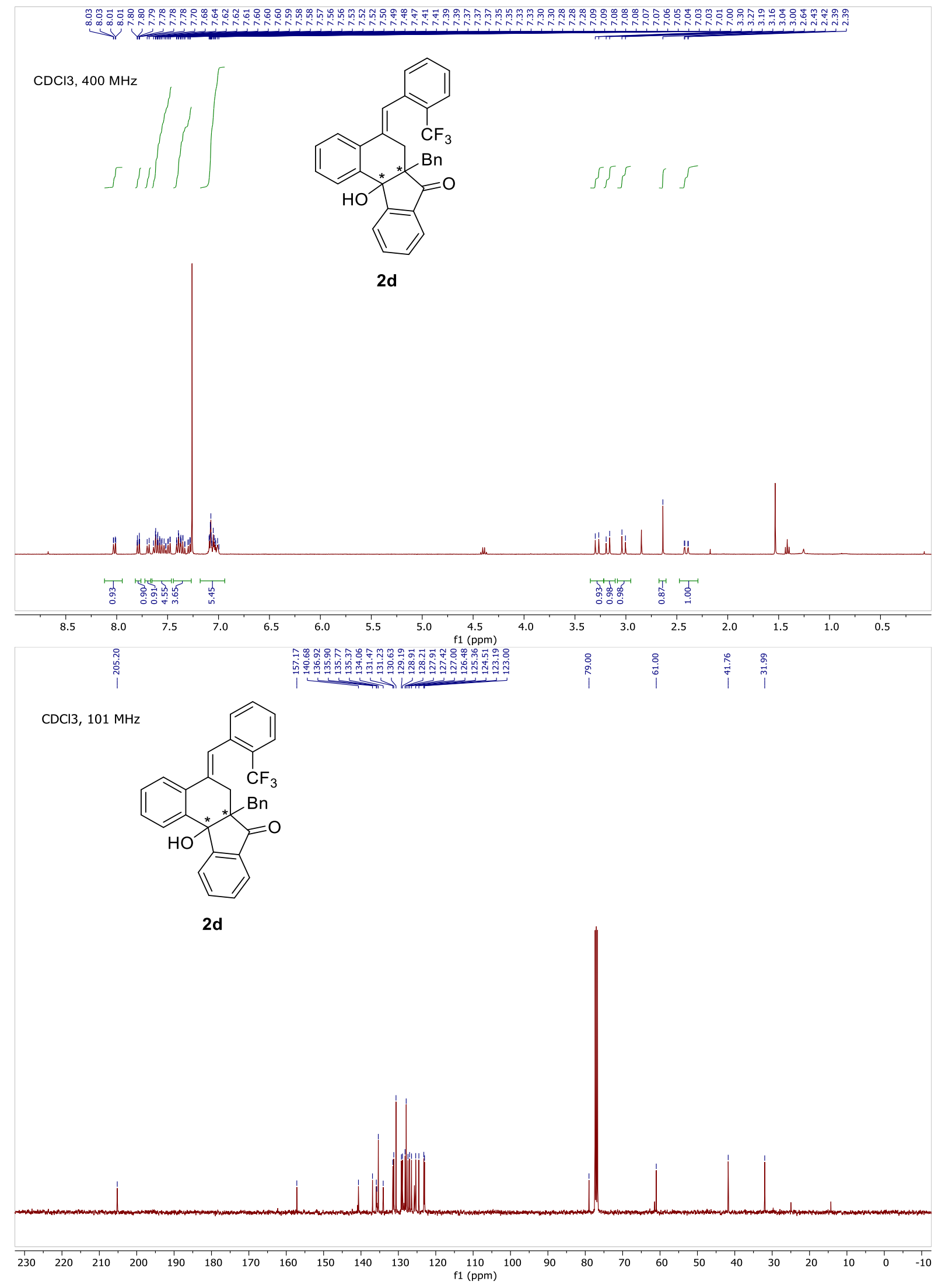


$\mathrm{CDCl} 3,400 \mathrm{MHz}$

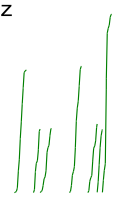<smiles>CCC1(C(C)=O)CC(=Cc2ccccc2C(F)(F)F)c2ccccc2C1(C)O</smiles>

Moldint

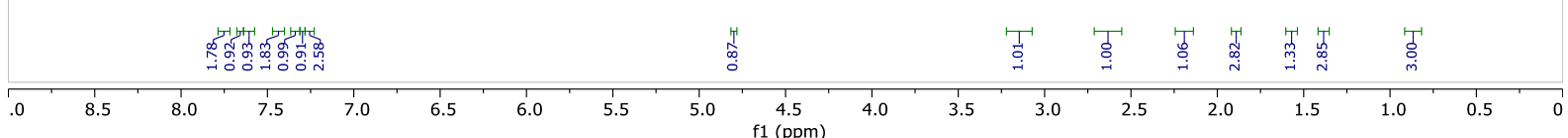

$\stackrel{\infty}{\stackrel{\infty}{N}}$

$\mathrm{CDCl} 3,101 \mathrm{MHz}$

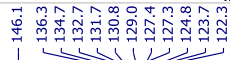<smiles>CCC1(C(C)=O)CC(=Cc2ccccc2C(F)(F)F)c2ccccc2C1(C)O</smiles>

2e

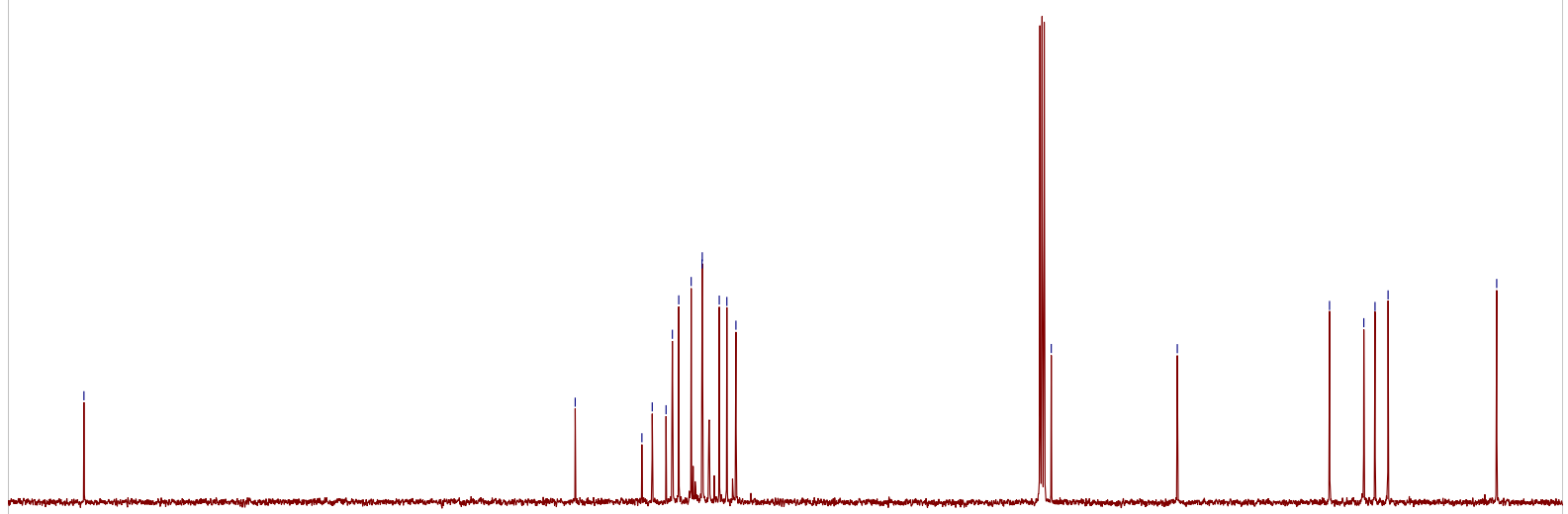

$30 \quad 220 \quad 210 \quad 200$
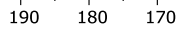

$160 \quad 150$

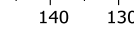

$\begin{array}{cc}120 & 110 \\ \mathrm{f} 1(\mathrm{ppm})\end{array}$ 


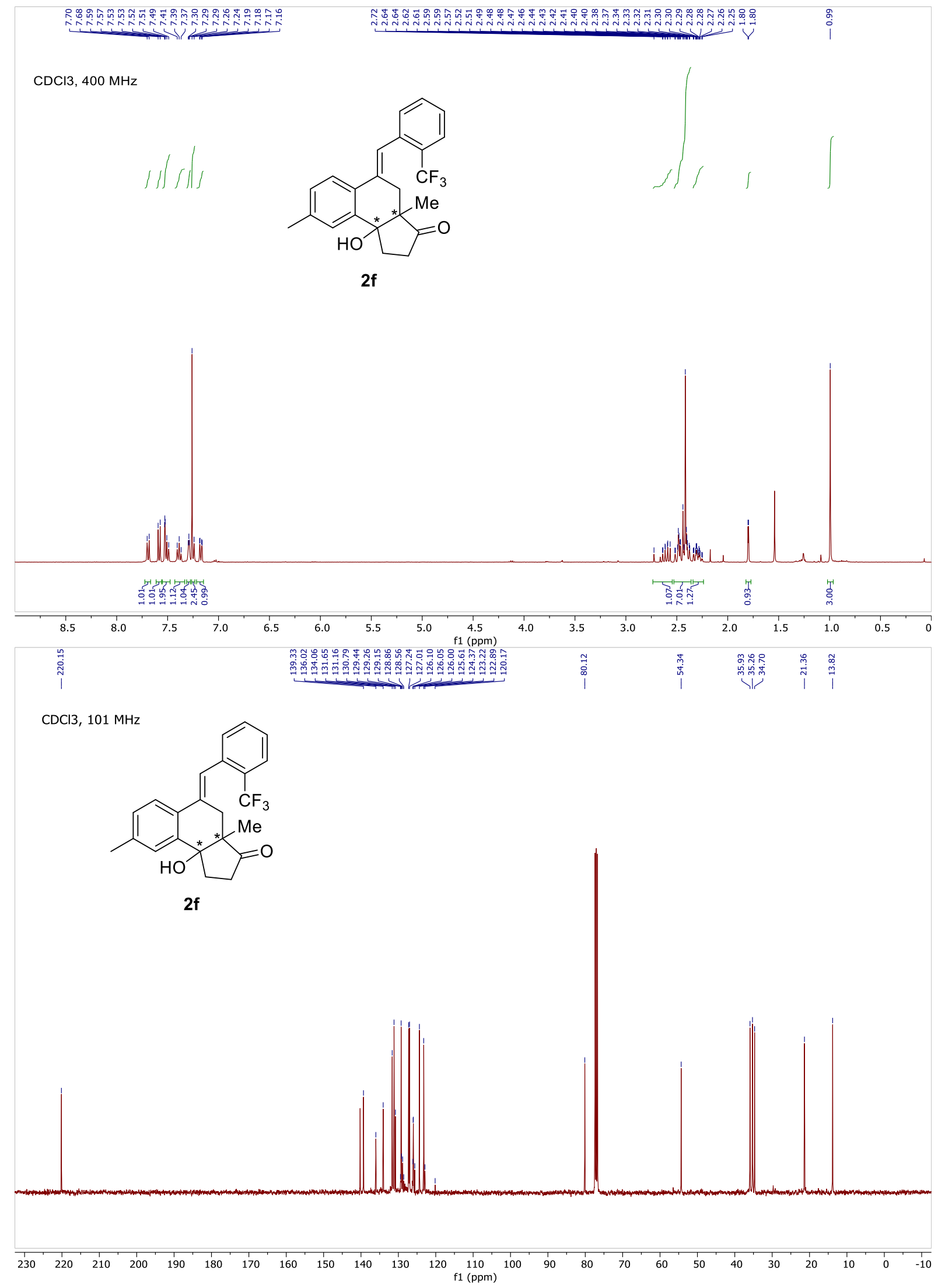



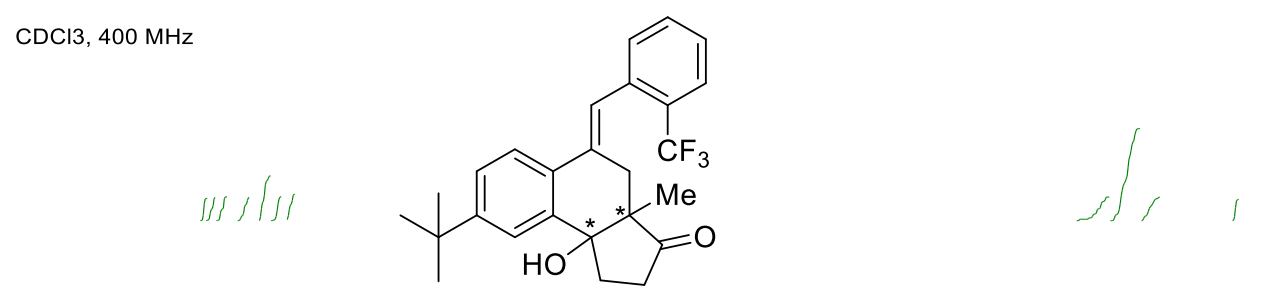

2g
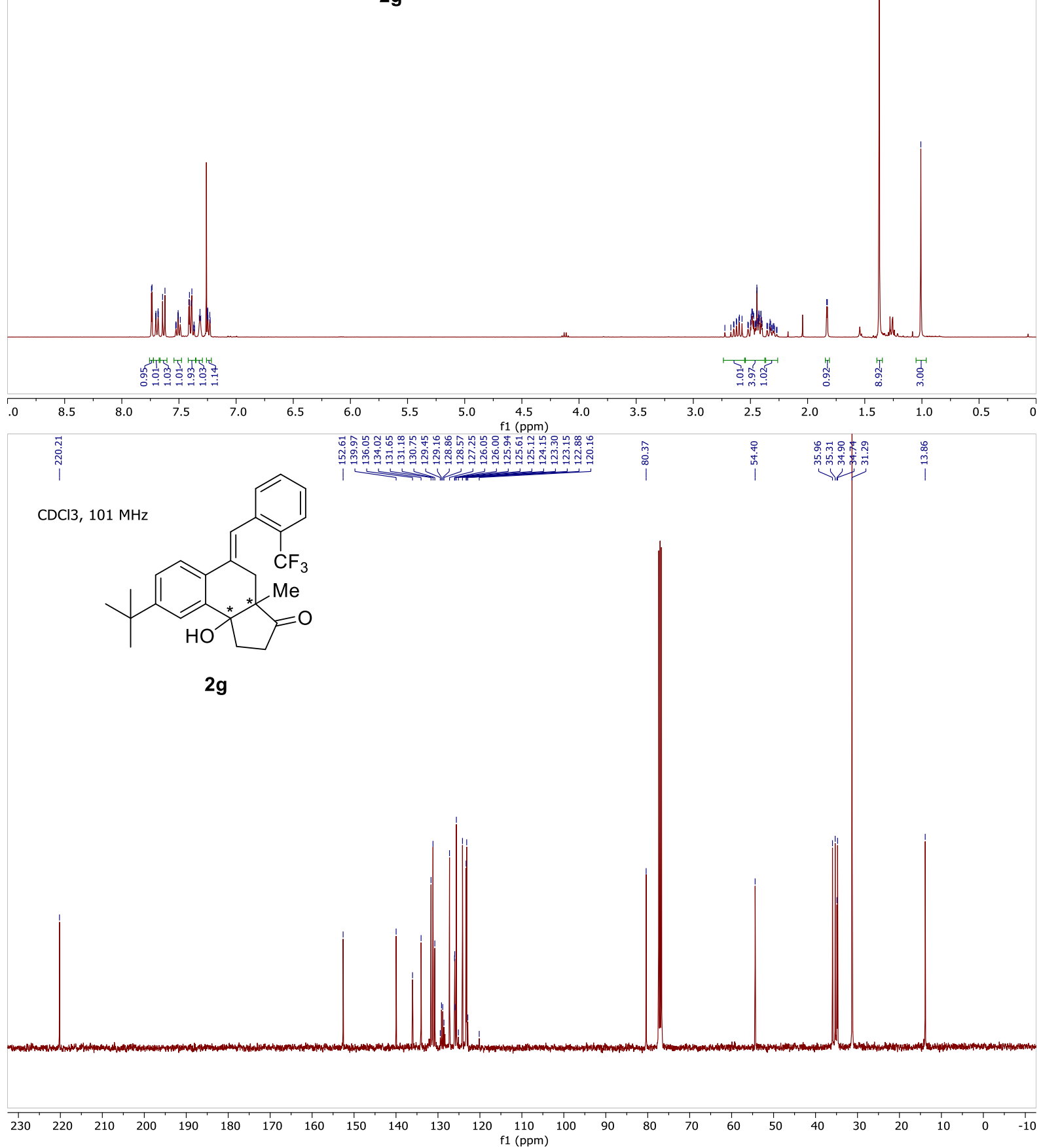

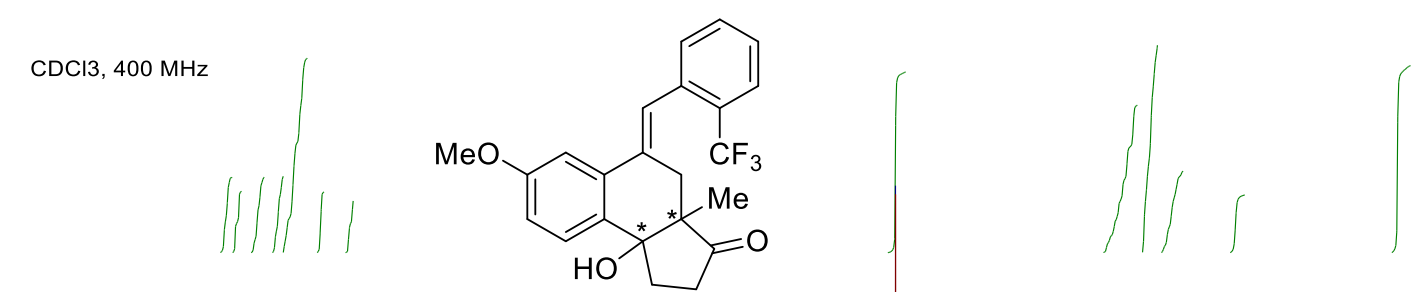

2h

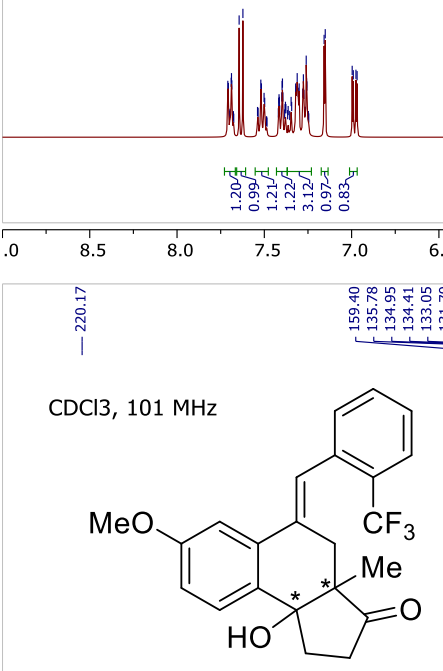

2h

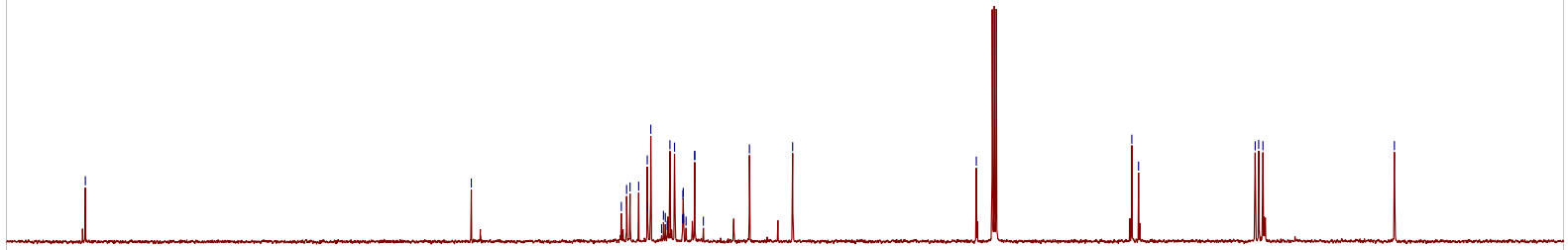

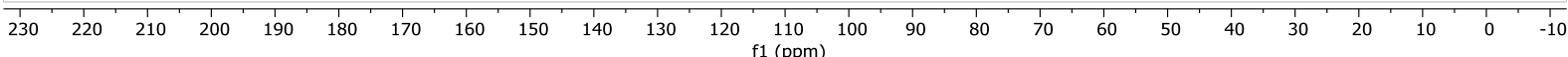




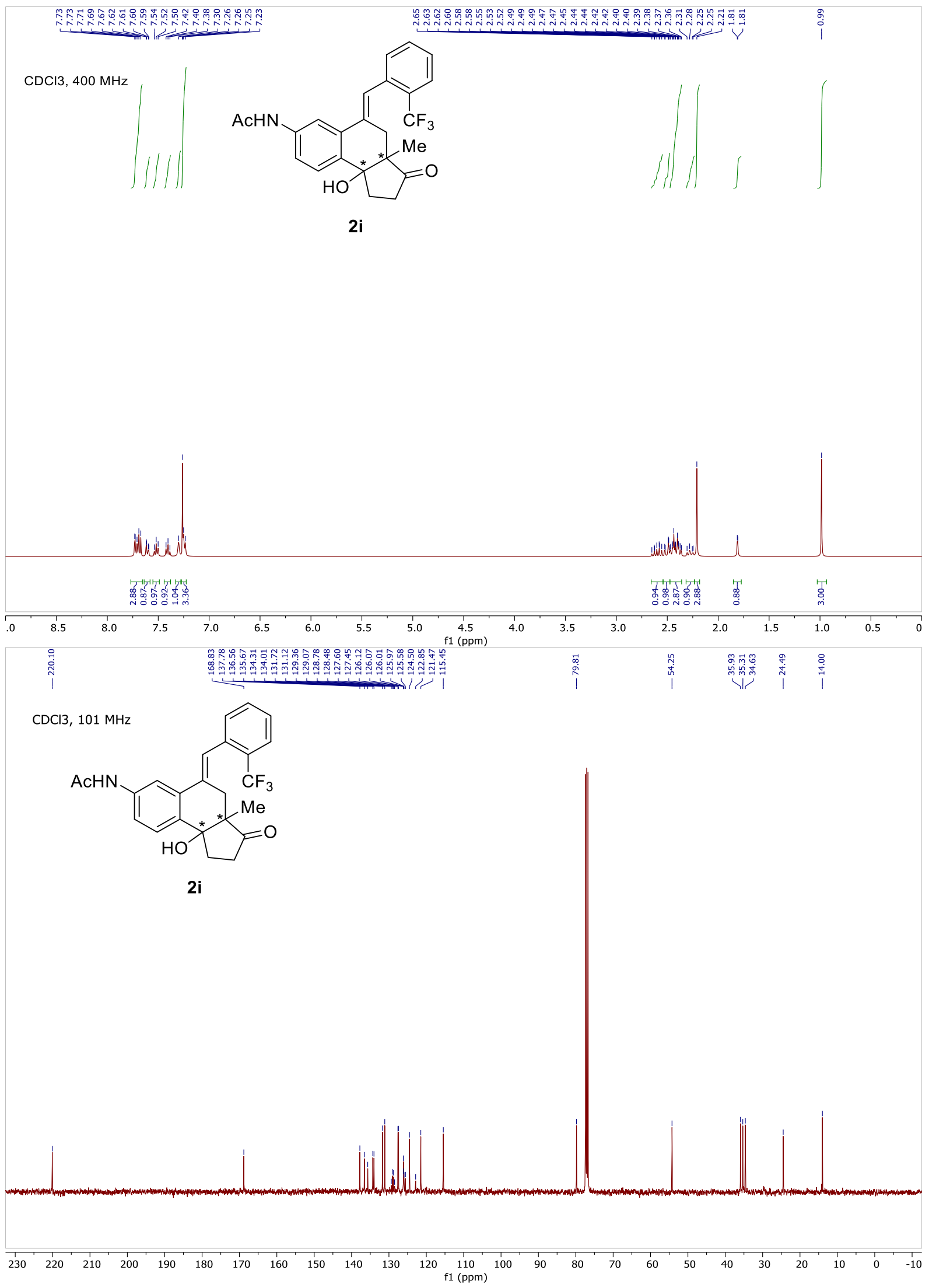




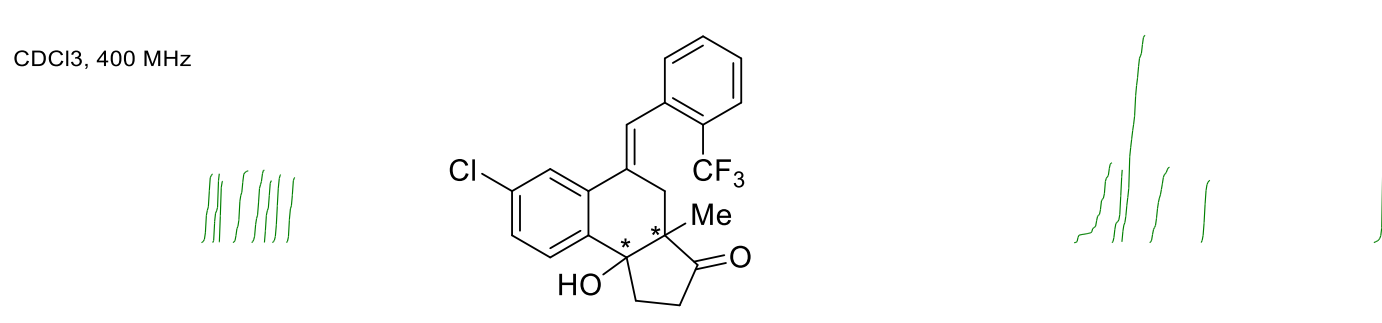

2j
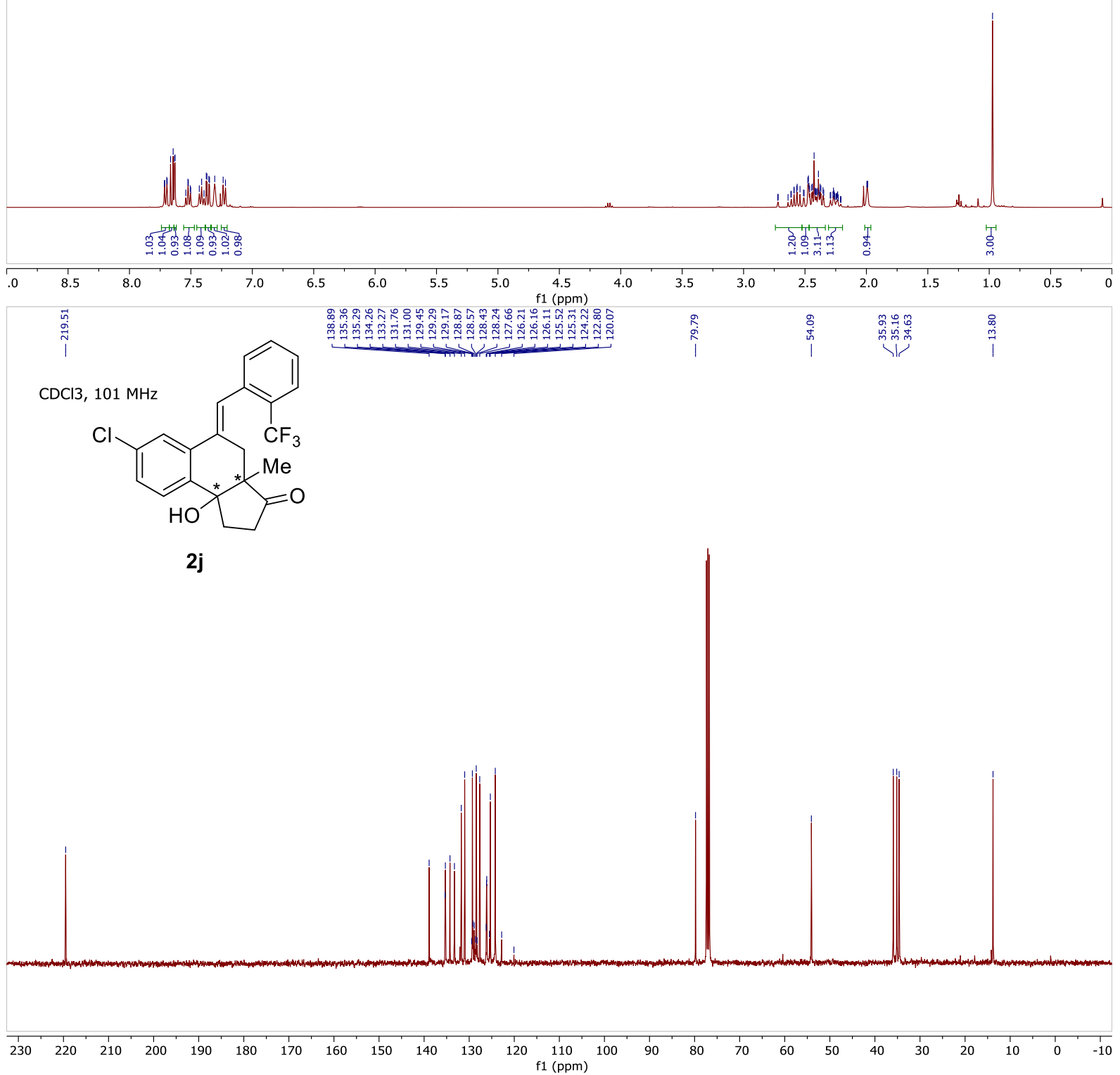

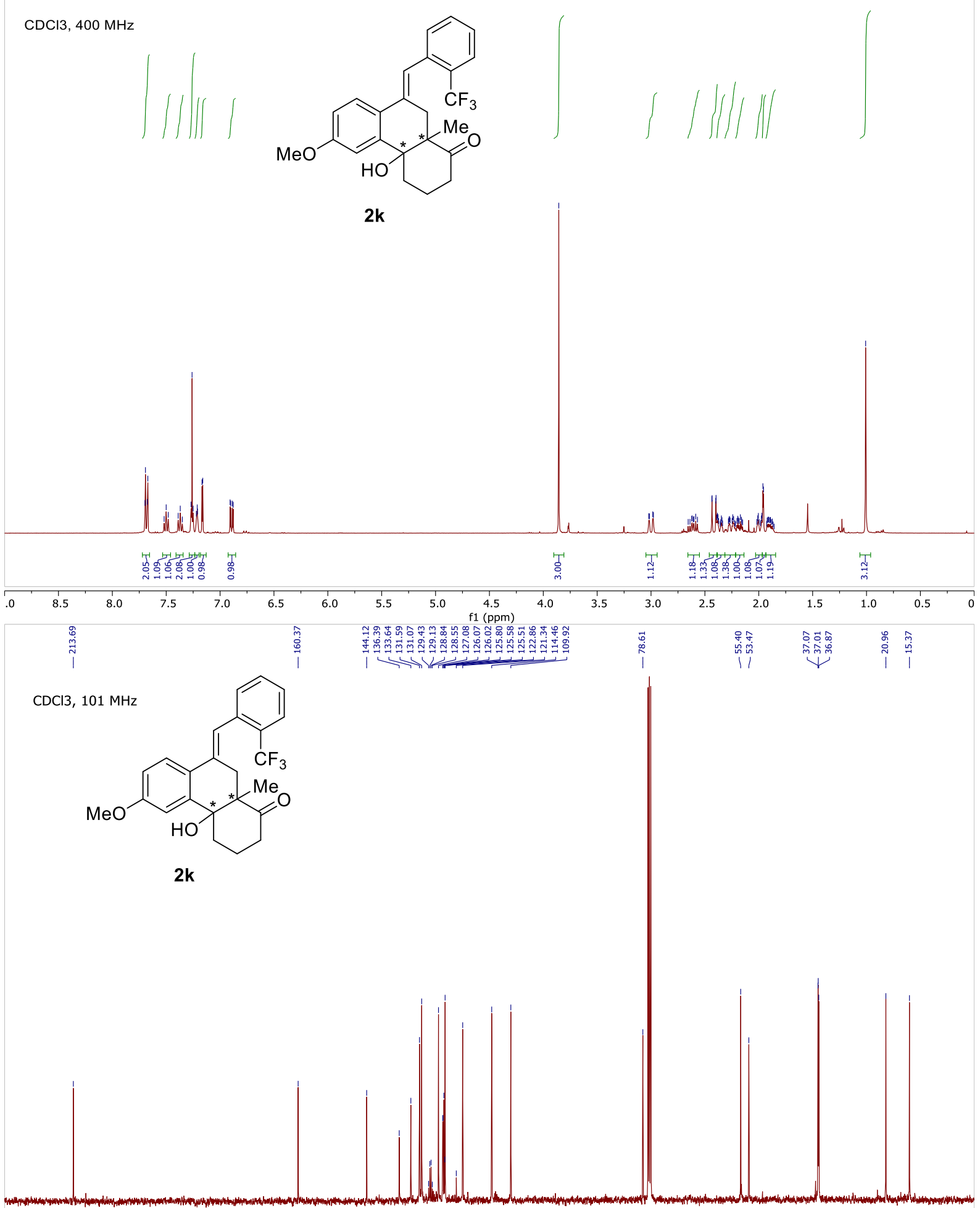

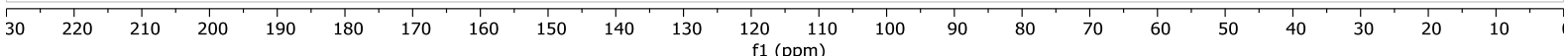


$\mathrm{CDCl} 3,400 \mathrm{MHz}$<smiles>C1CCCCC1</smiles><smiles>CC(C)(C)c1ccc2c(c1)CC1(O)C(=O)CCCC1(O)C2=Cc1ccccc1F</smiles>

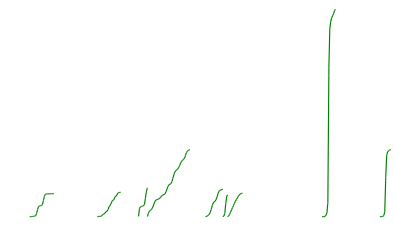

2)

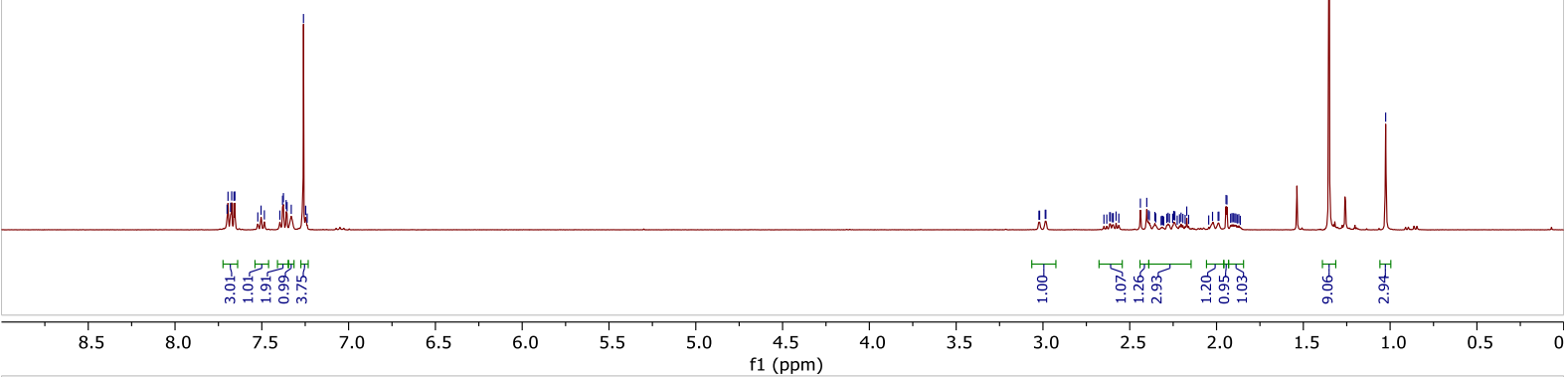<smiles>CC(C)(C)c1ccc2c(c1)[C@]1(O)CCCC(=O)[C@@]1(C)C/C2=C\c1ccccc1C(C)(C)C</smiles>

|

21

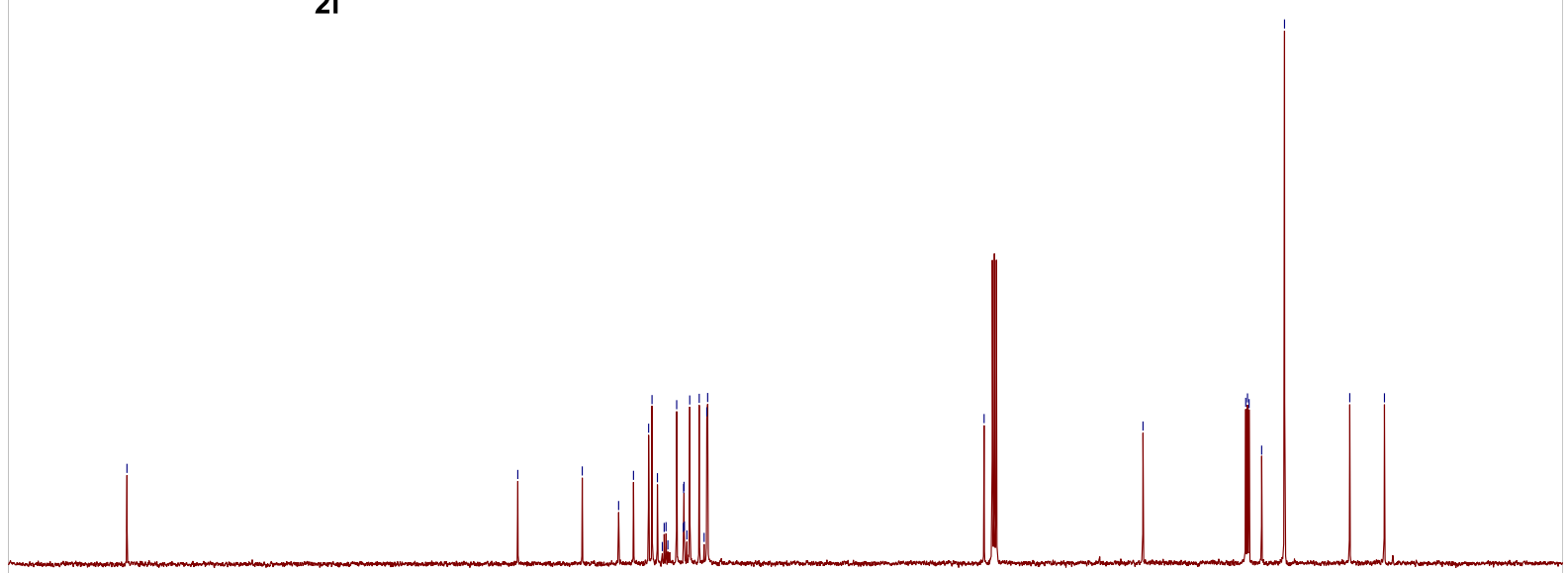

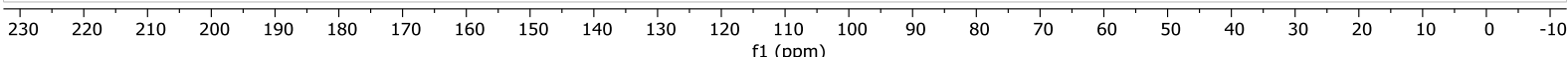



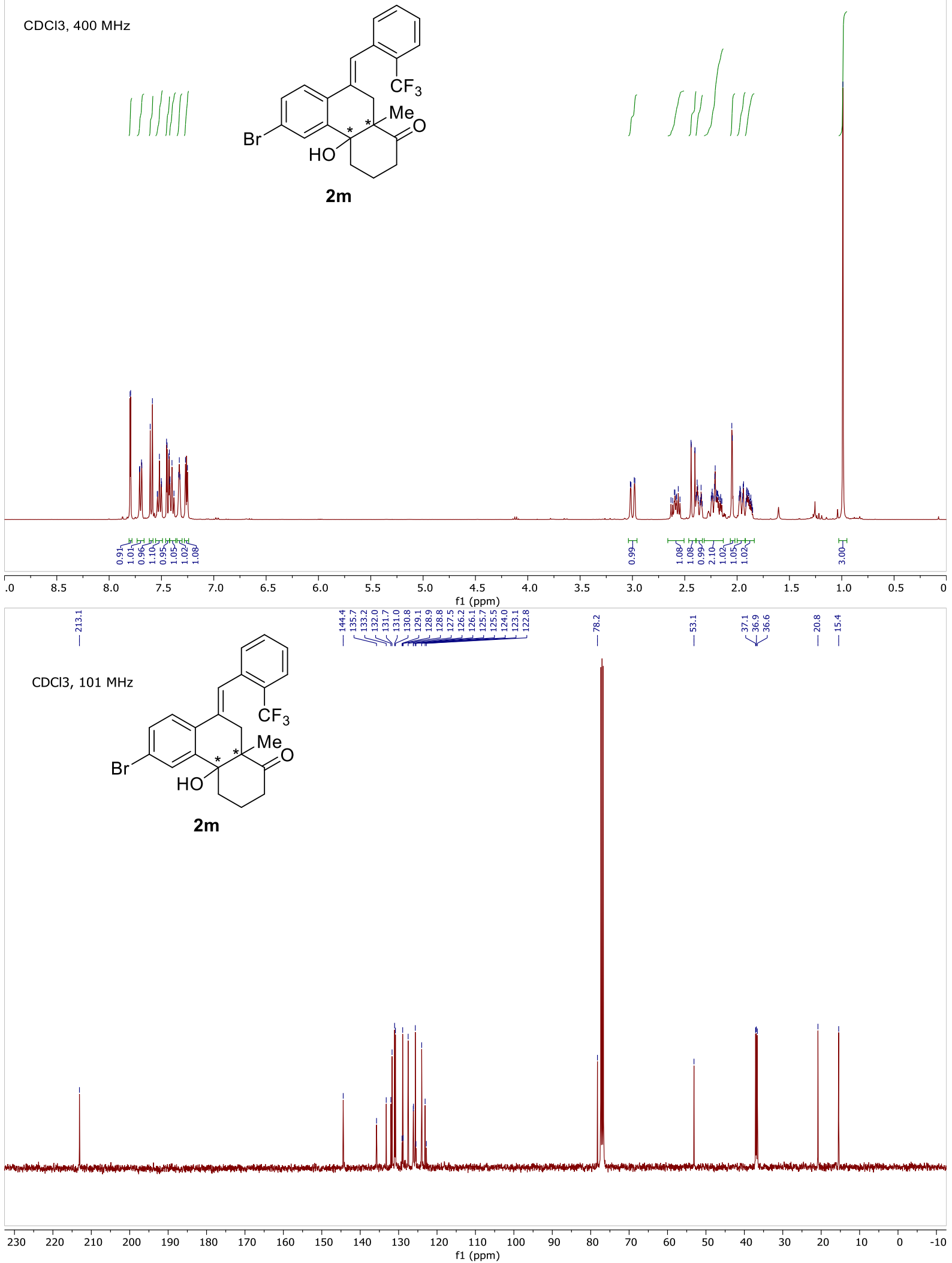


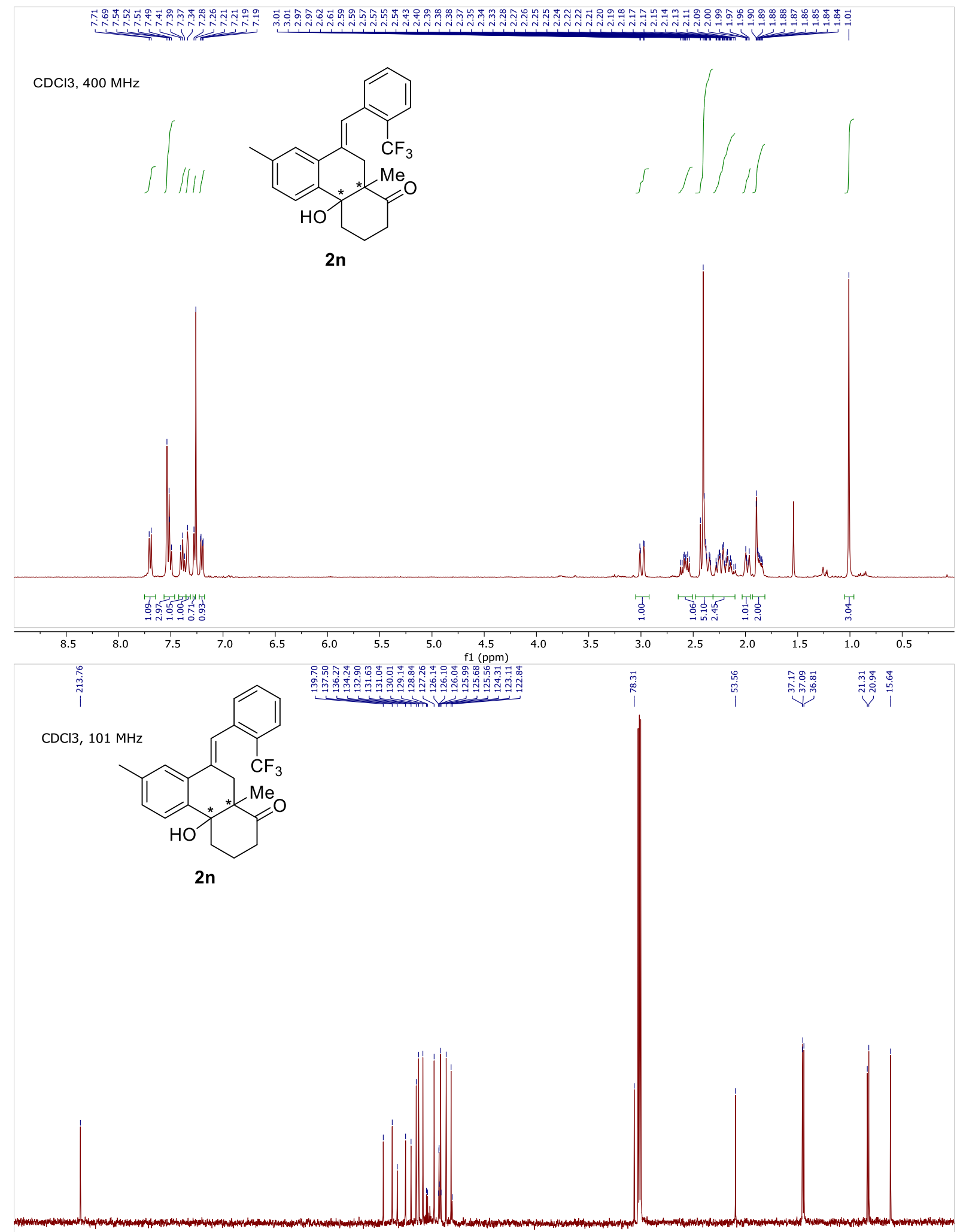

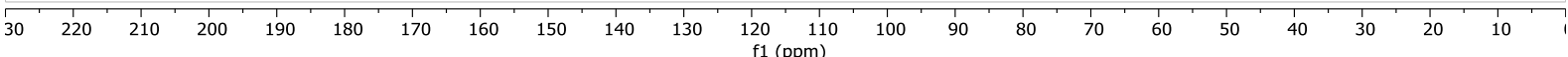


$\mathrm{CDCl} 3,400 \mathrm{MHz}$

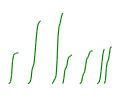<smiles>CC(C)(C)c1ccc2c(c1)C(=Cc1ccccc1F)CC1(O)CCCC(=O)C21O</smiles>

20
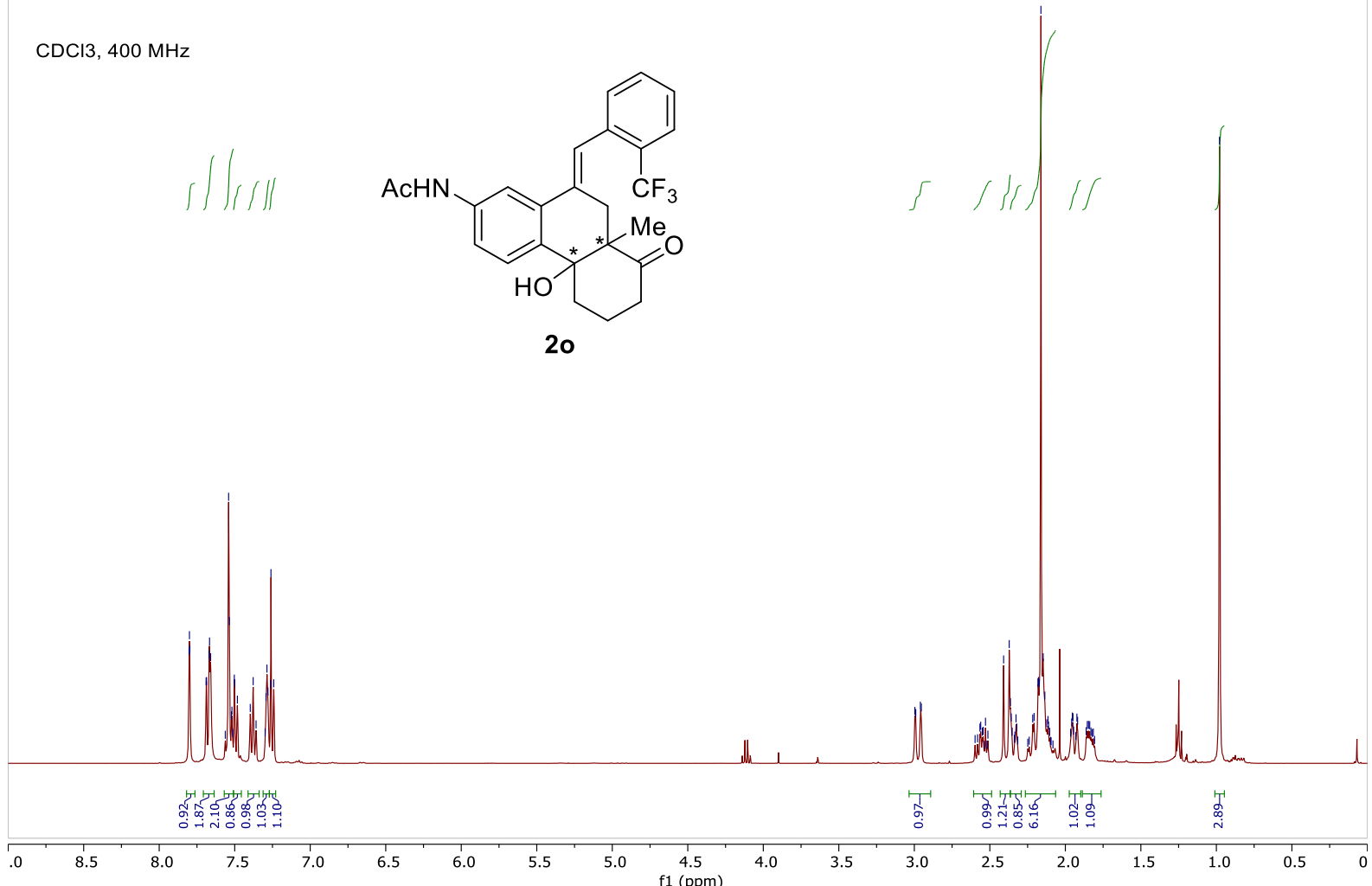

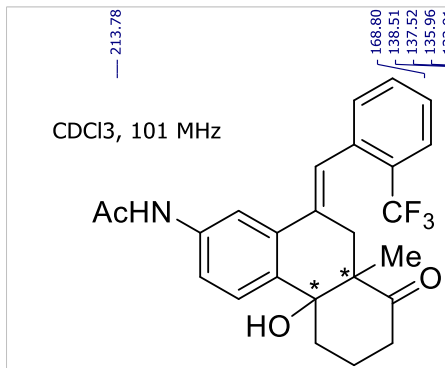

20

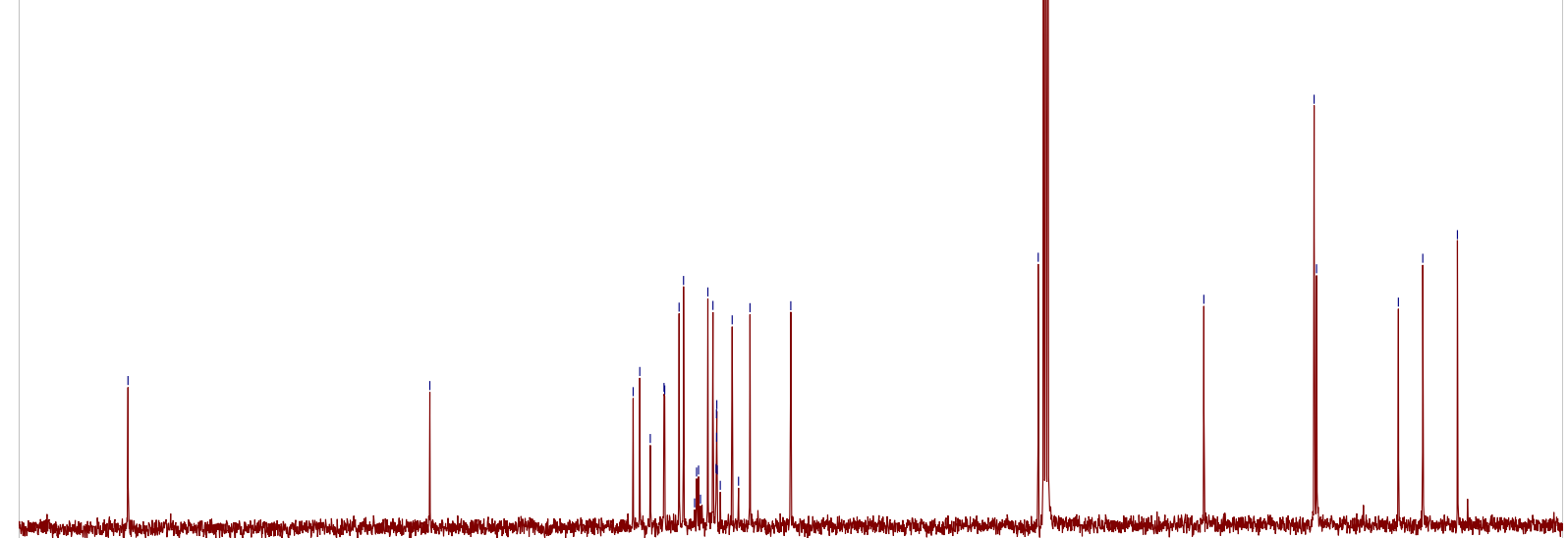

\begin{tabular}{|l|llllllllllllllllllllll}
\hline 30 & 220 & 210 & 200 & 190 & 180 & 170 & 160 & 150 & 140 & 130 & $\begin{array}{c}120 \\
\mathrm{f} 1(\mathrm{ppm})\end{array}$ & 110 & 100 & 90 & 80 & 70 & 60 & 50 & 40 & 30 & 20 & 10 \\
\hline
\end{tabular} 

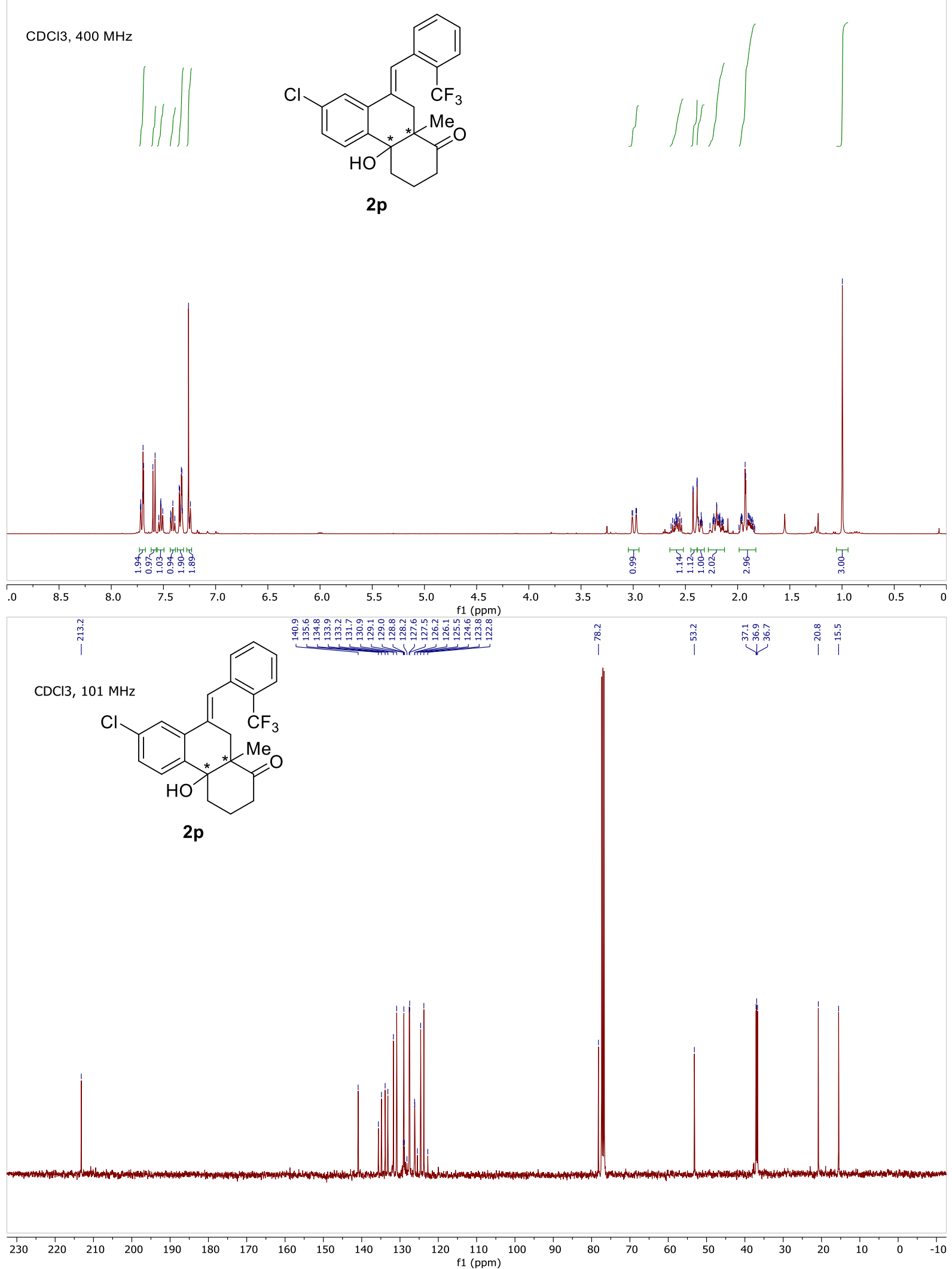

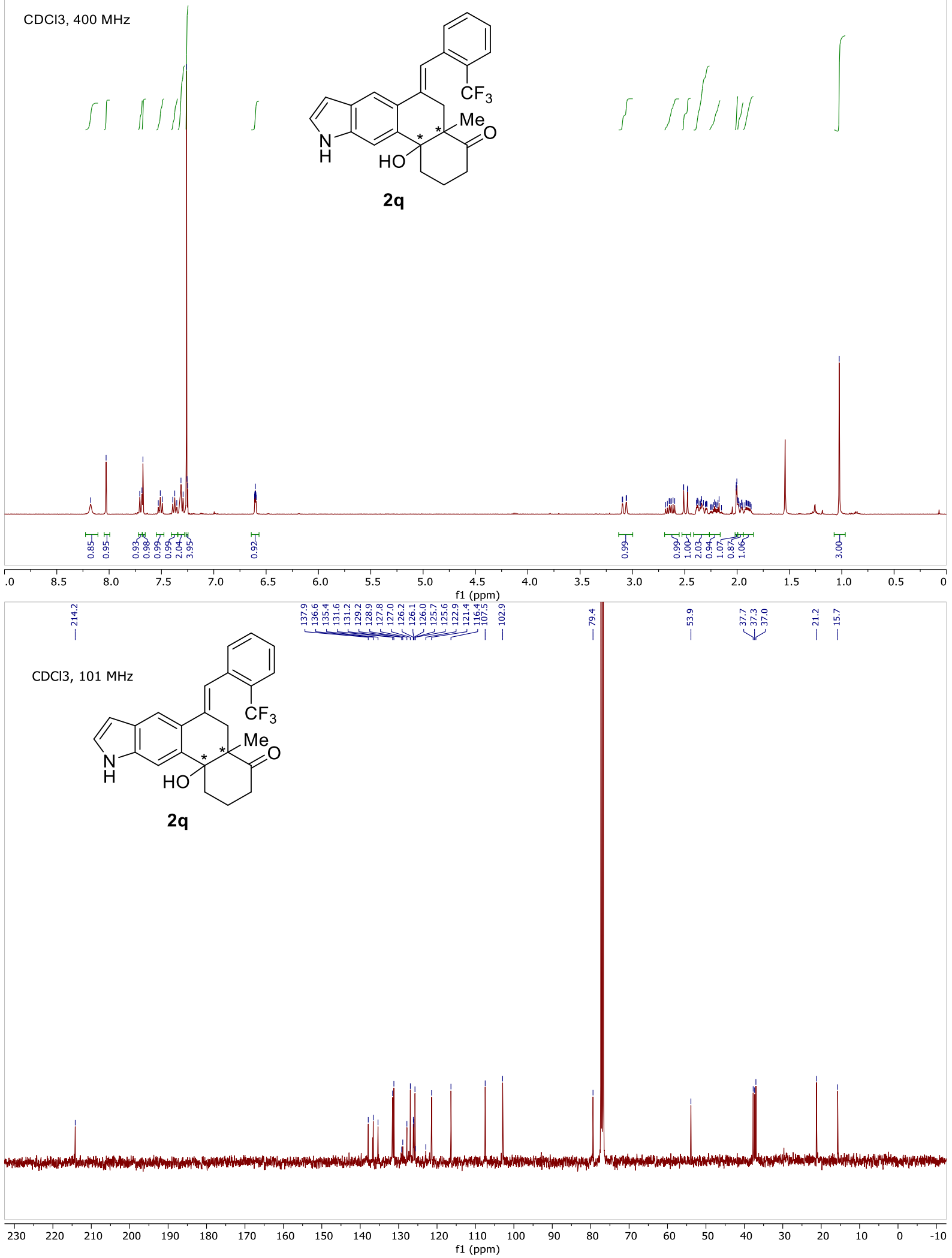


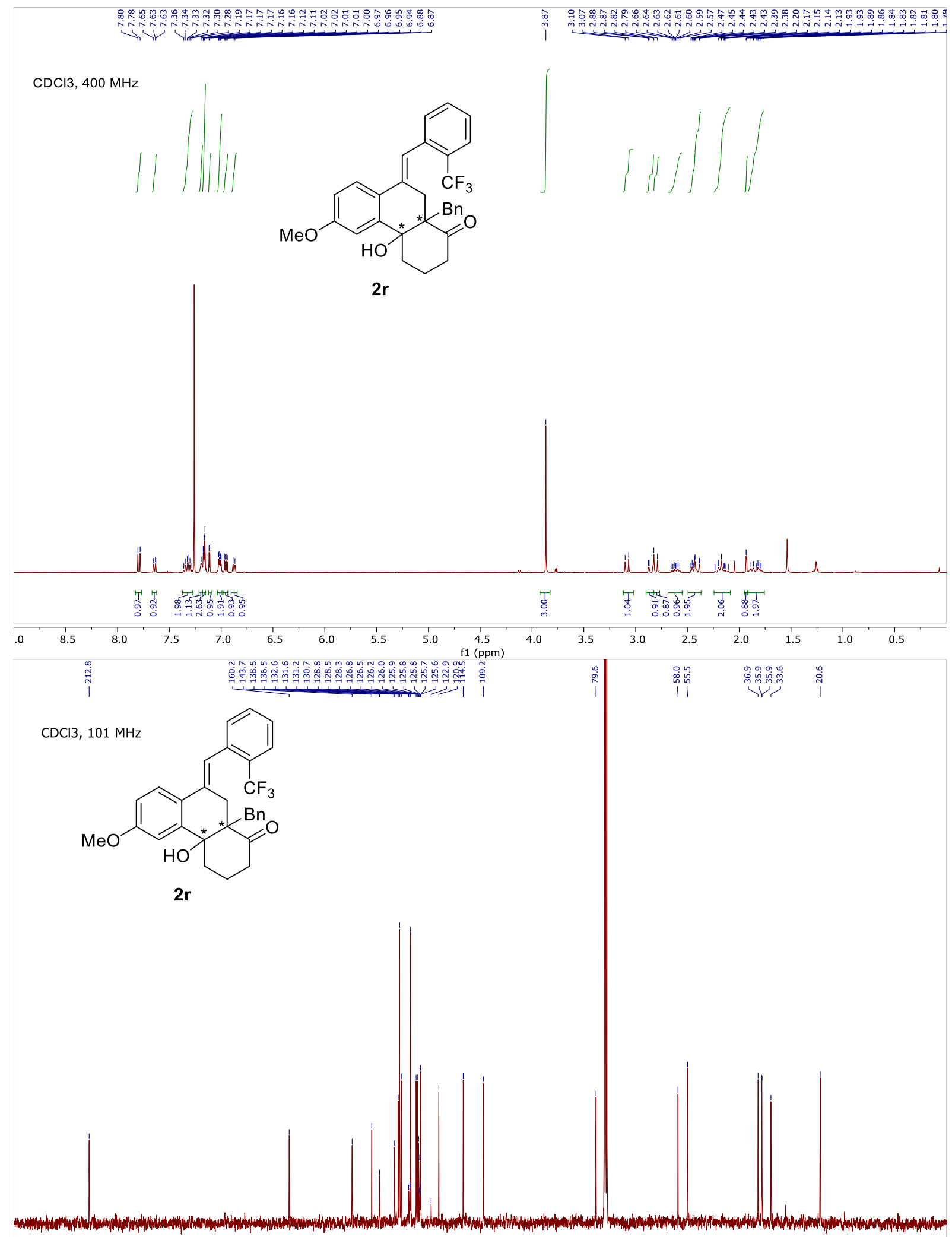

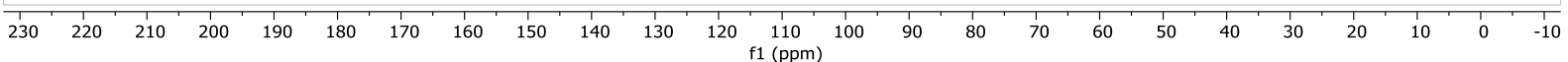


$\mathrm{CDCl} 3,400 \mathrm{MHz}$

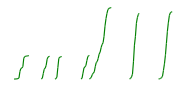<smiles>CC(C)(C)c1ccc2c(c1)CC1(O)C(=O)CCCC1(Br)C2=Cc1ccccc1F</smiles>

2s
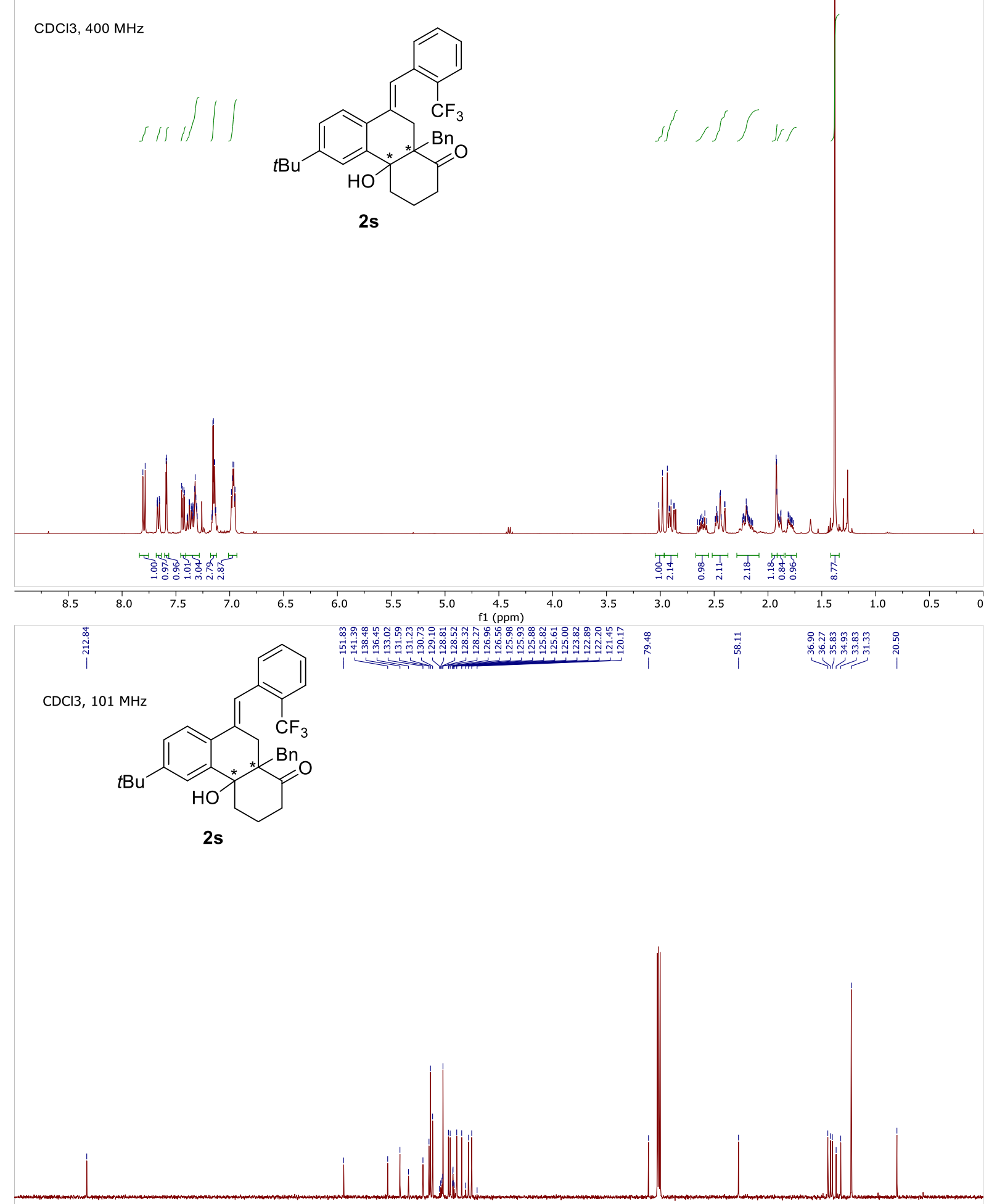

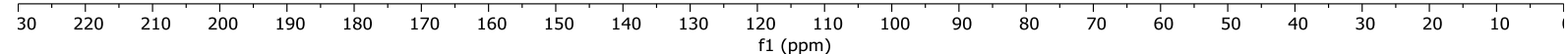




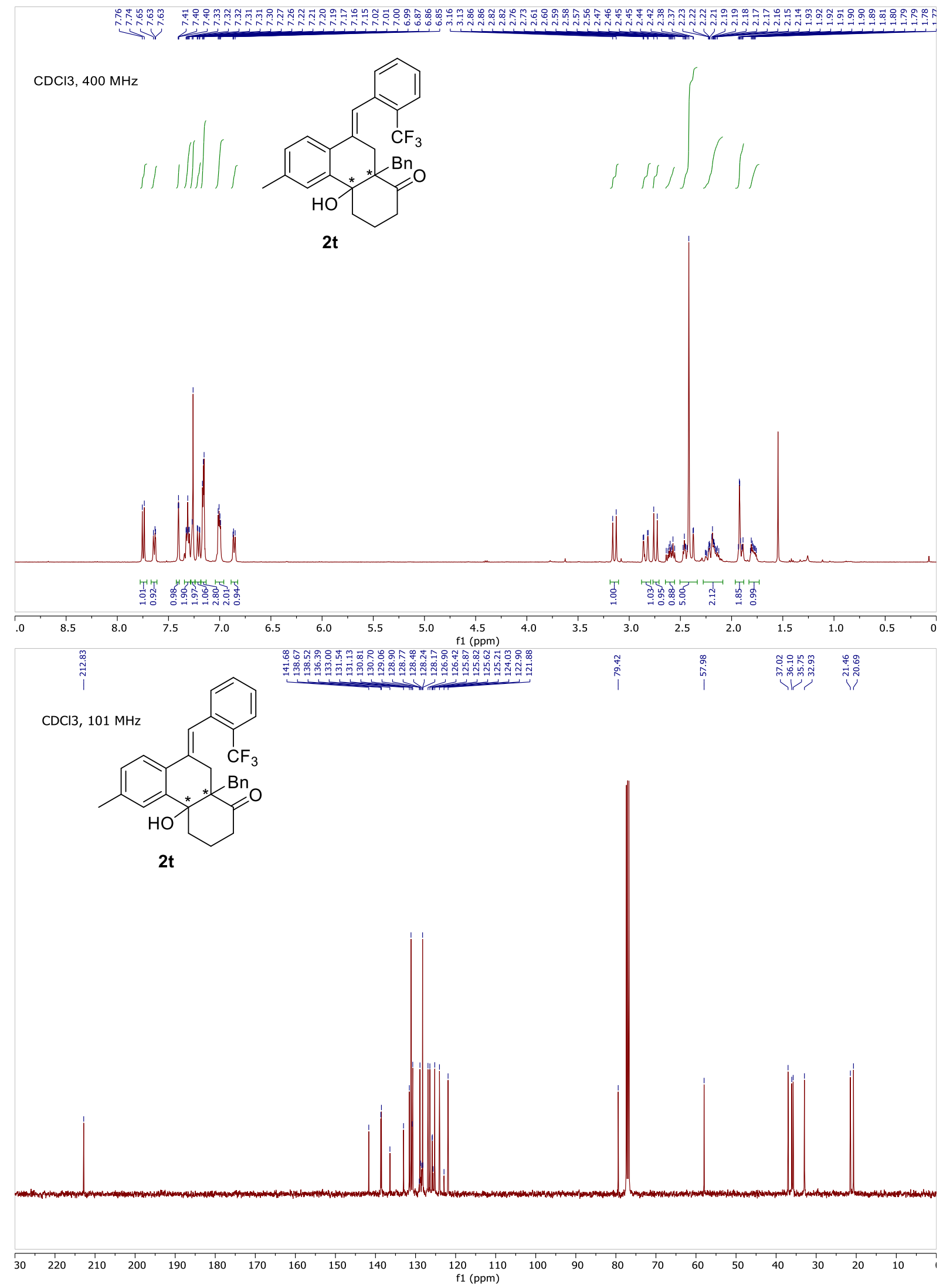



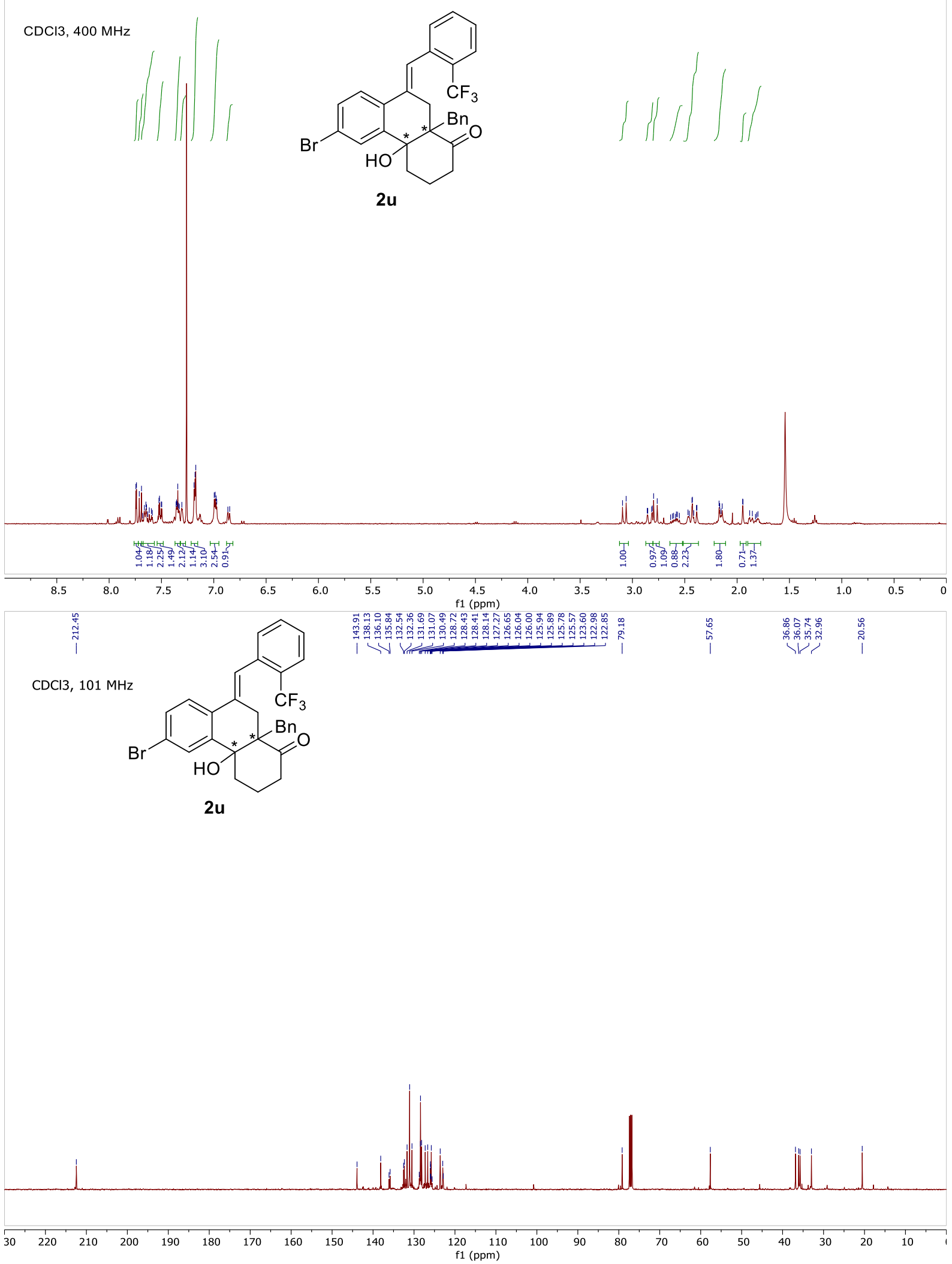


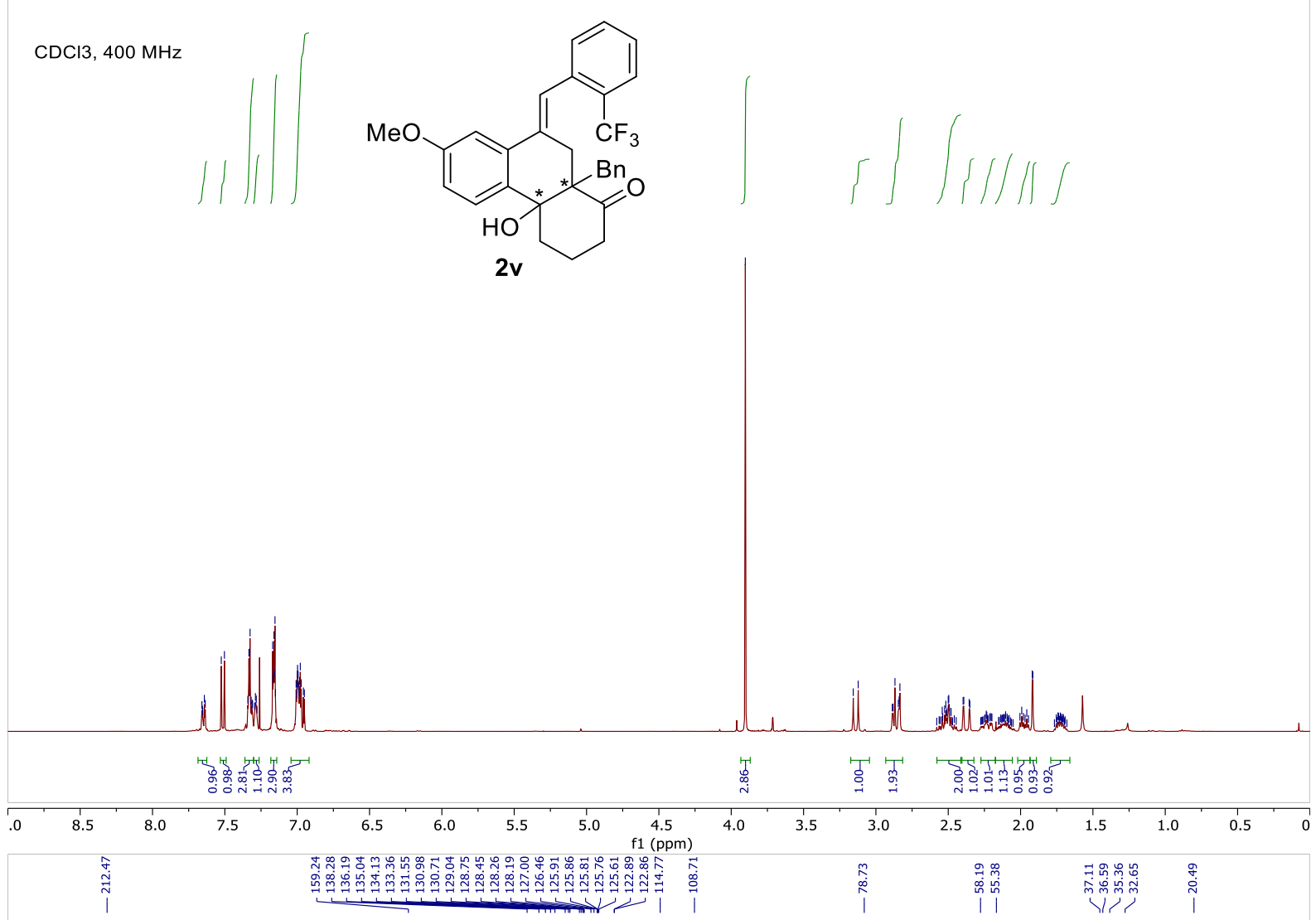

$\mathrm{CDCl} 3,101 \mathrm{MHz}$<smiles>COc1ccc2c(c1)/C(=C/c1ccccc1C(F)(F)F)CC1(O)C(=O)CCCC21O</smiles>
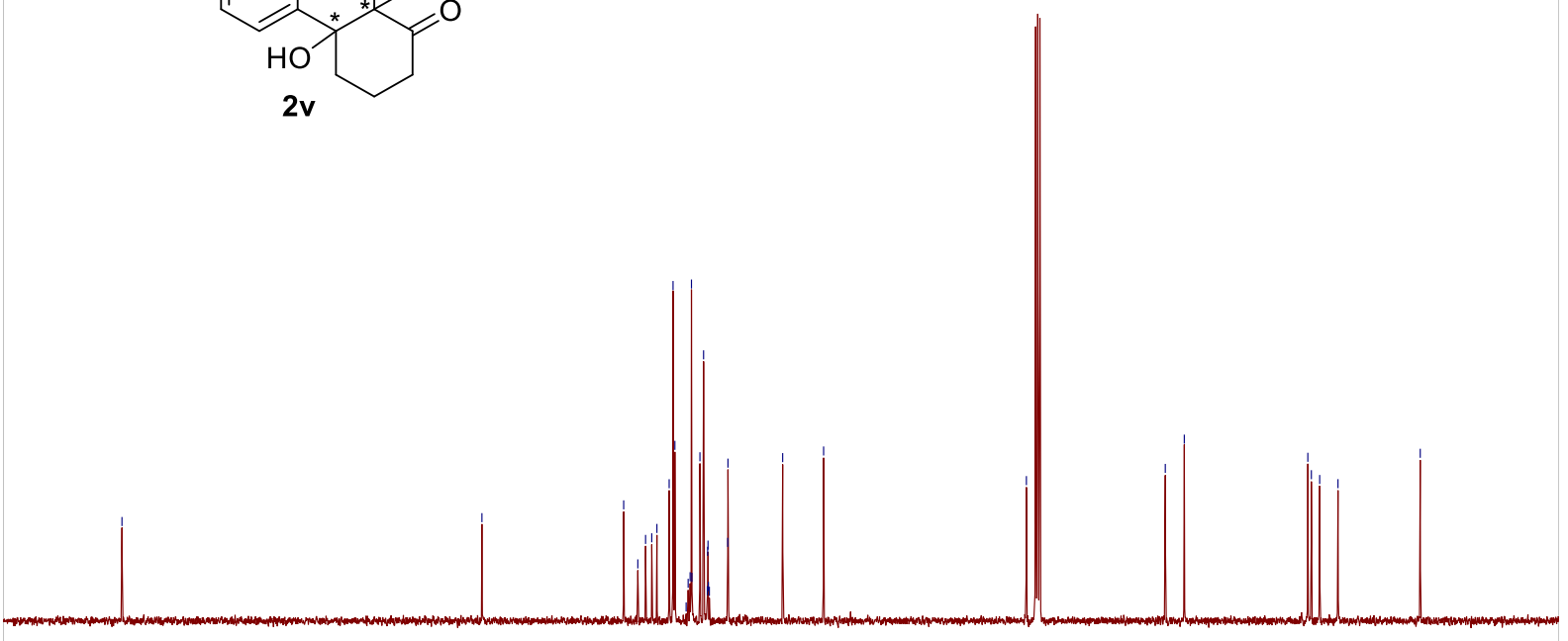

\begin{tabular}{lllllllllllllllllllllllll}
\hline 30 & 220 & 210 & 200 & 190 & 180 & 170 & 160 & 150 & 140 & 130 & 120 & 110 & 100 & 90 & 80 & 70 & 60 & 50 & 40 & 30 & 20 & 10 & 10
\end{tabular} 

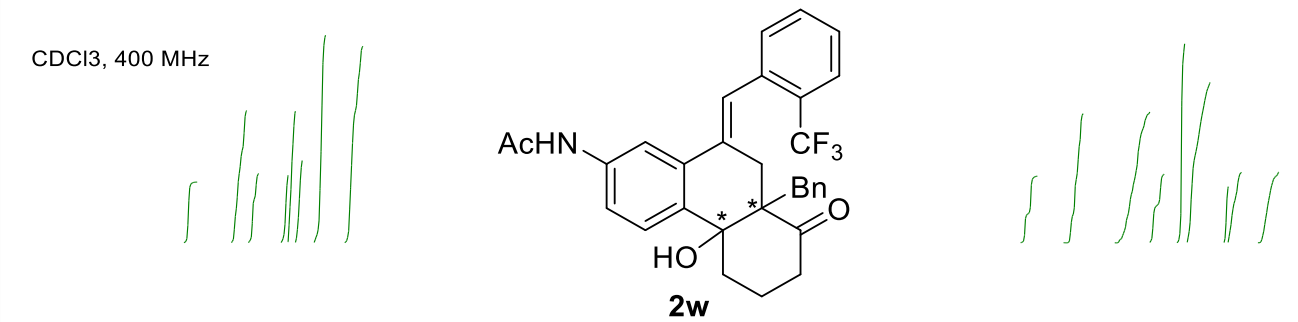

2w
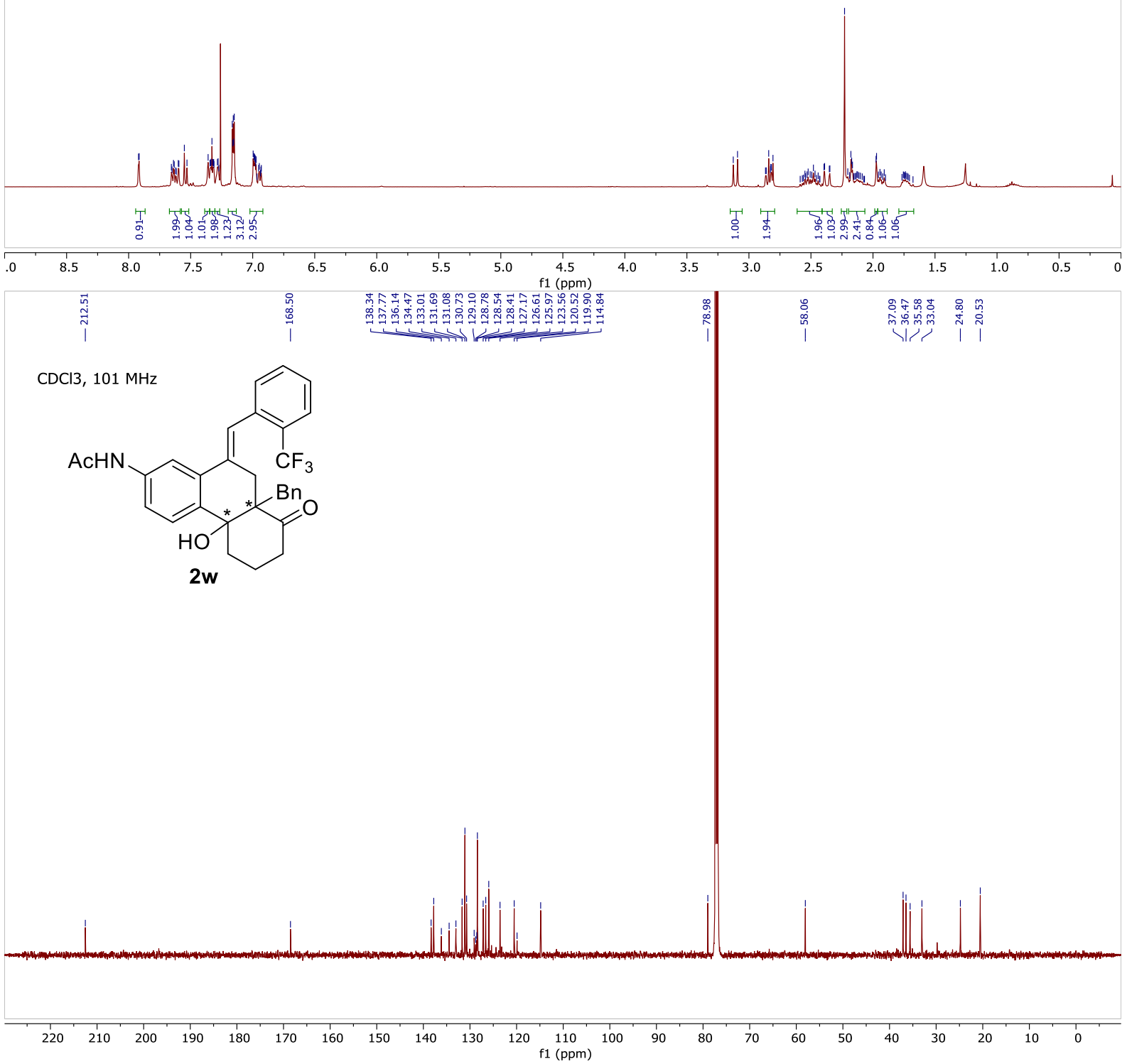


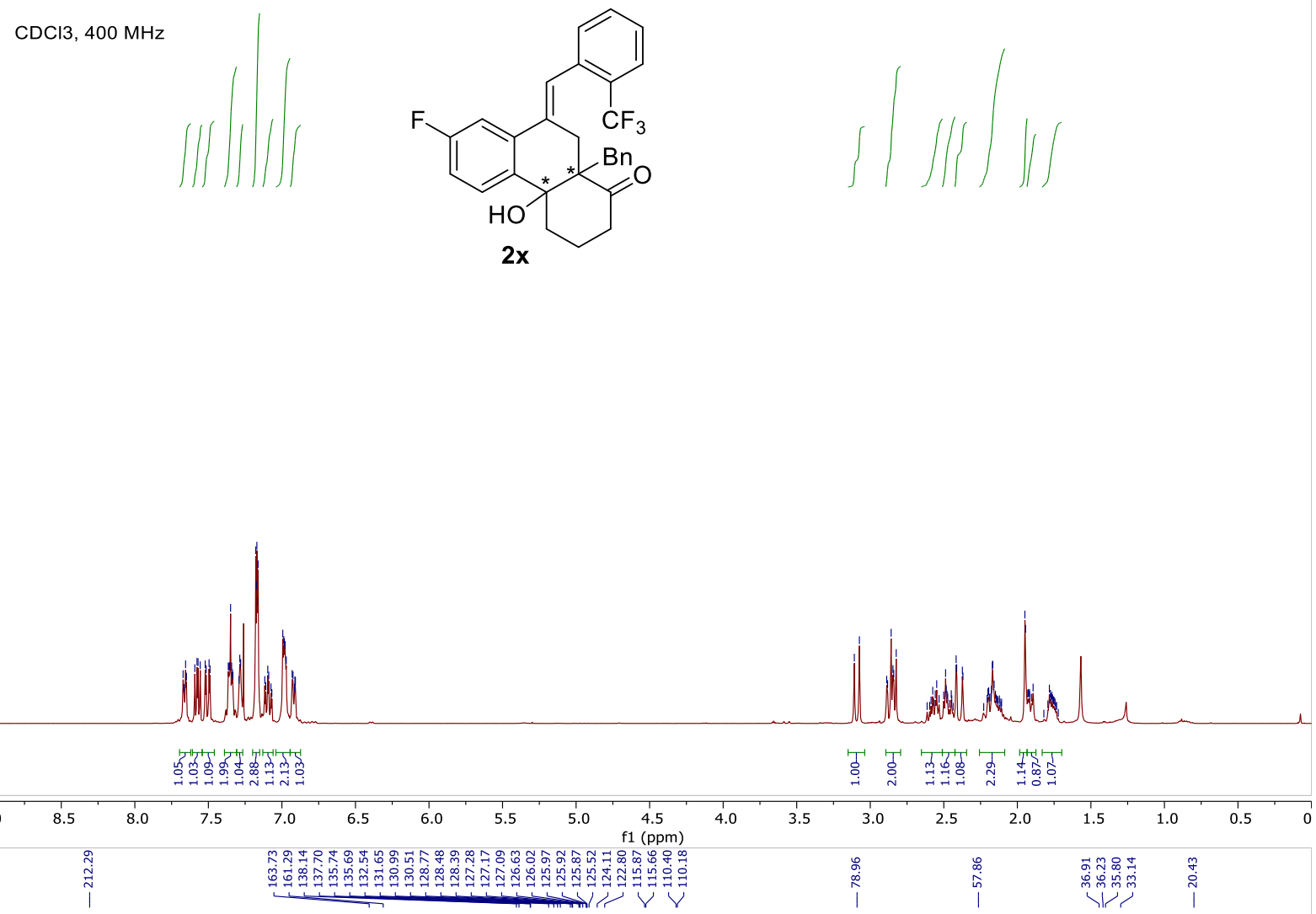

$\mathrm{CDCl} 3,101 \mathrm{MHz}$

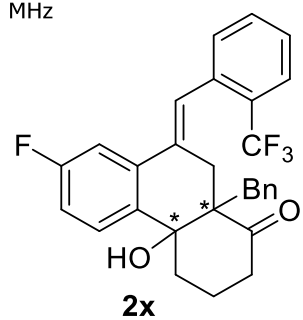

\begin{tabular}{lllllllllllll}
\hline 30 & 220 & 210 & 200 & 190 & 180 & 170 & 160 & 150 & 140 & 130 & 120 & 110
\end{tabular} 


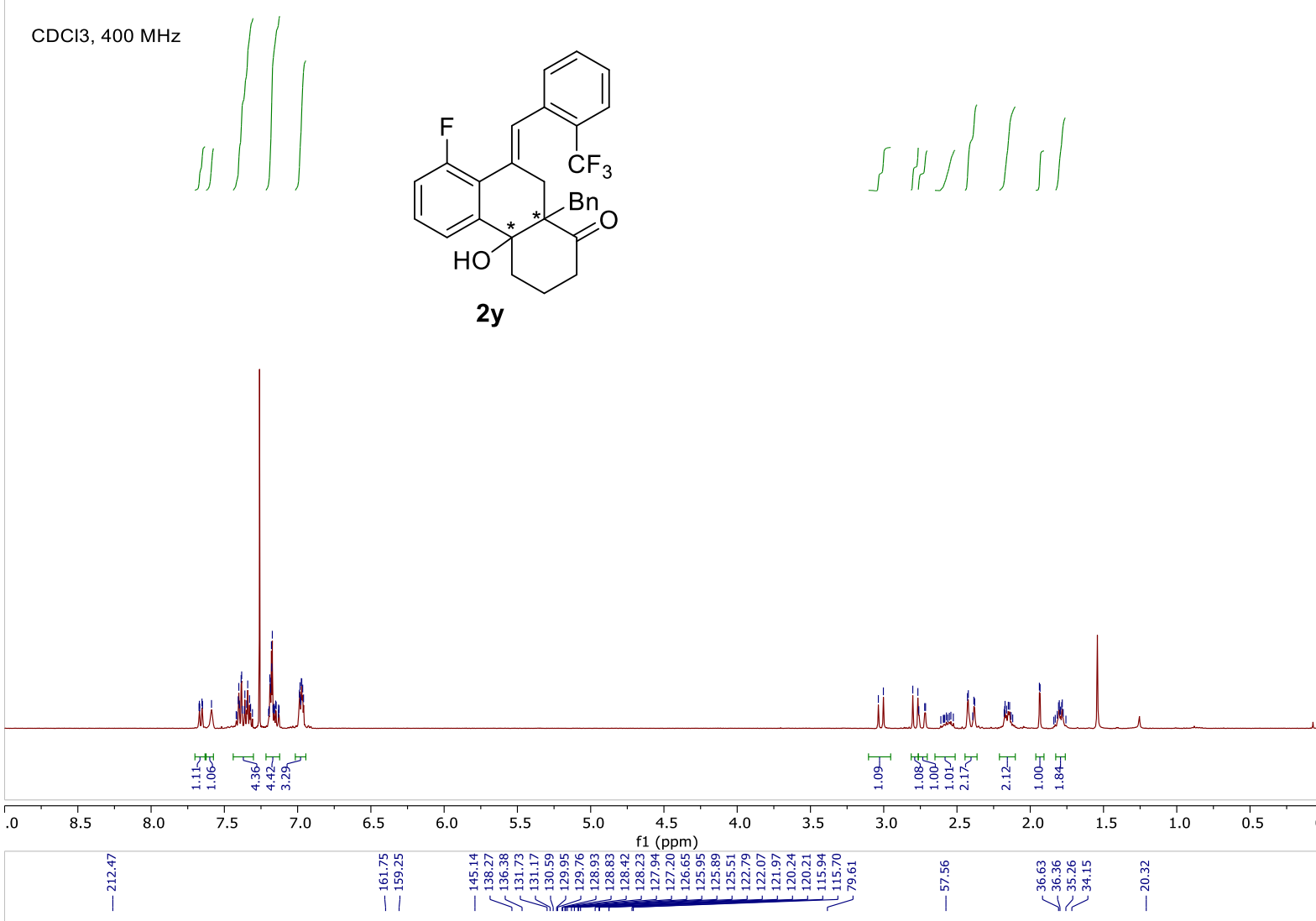

$\mathrm{CDCl}, 101 \mathrm{MHz}$<smiles>O=C1CCC[C@](O)(Br)c2cccc(F)c2/C1=C\c1ccccc1C(F)(F)F</smiles>

$2 y$

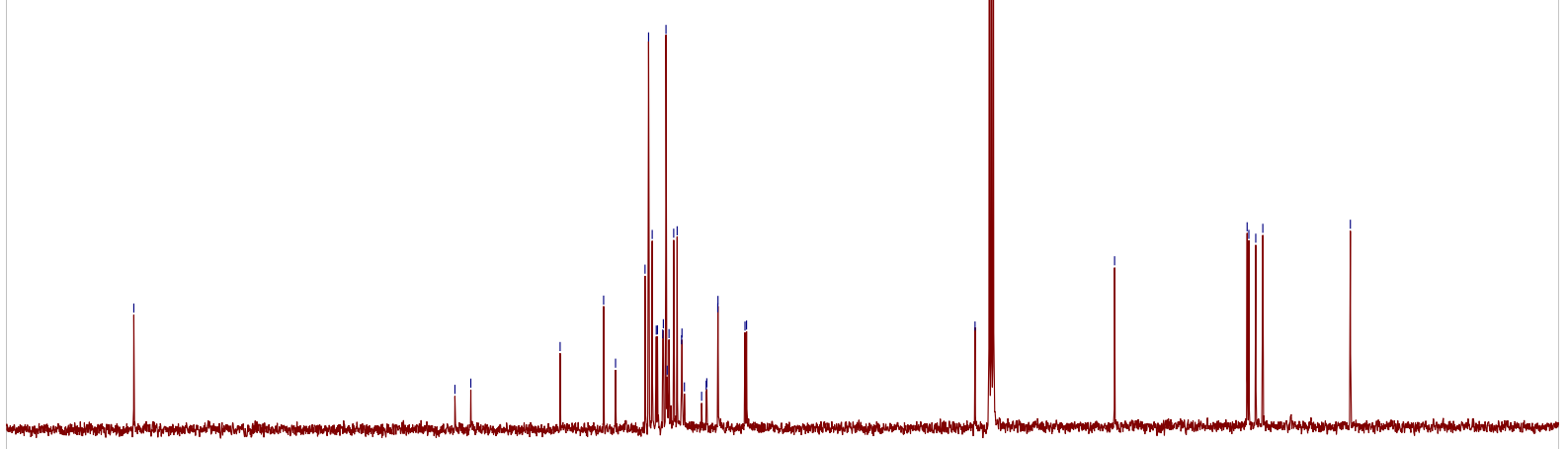

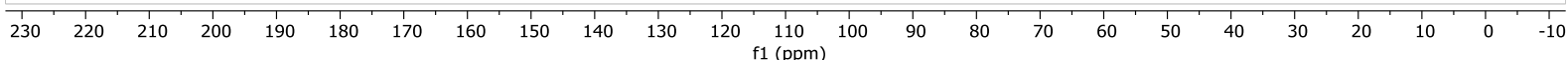




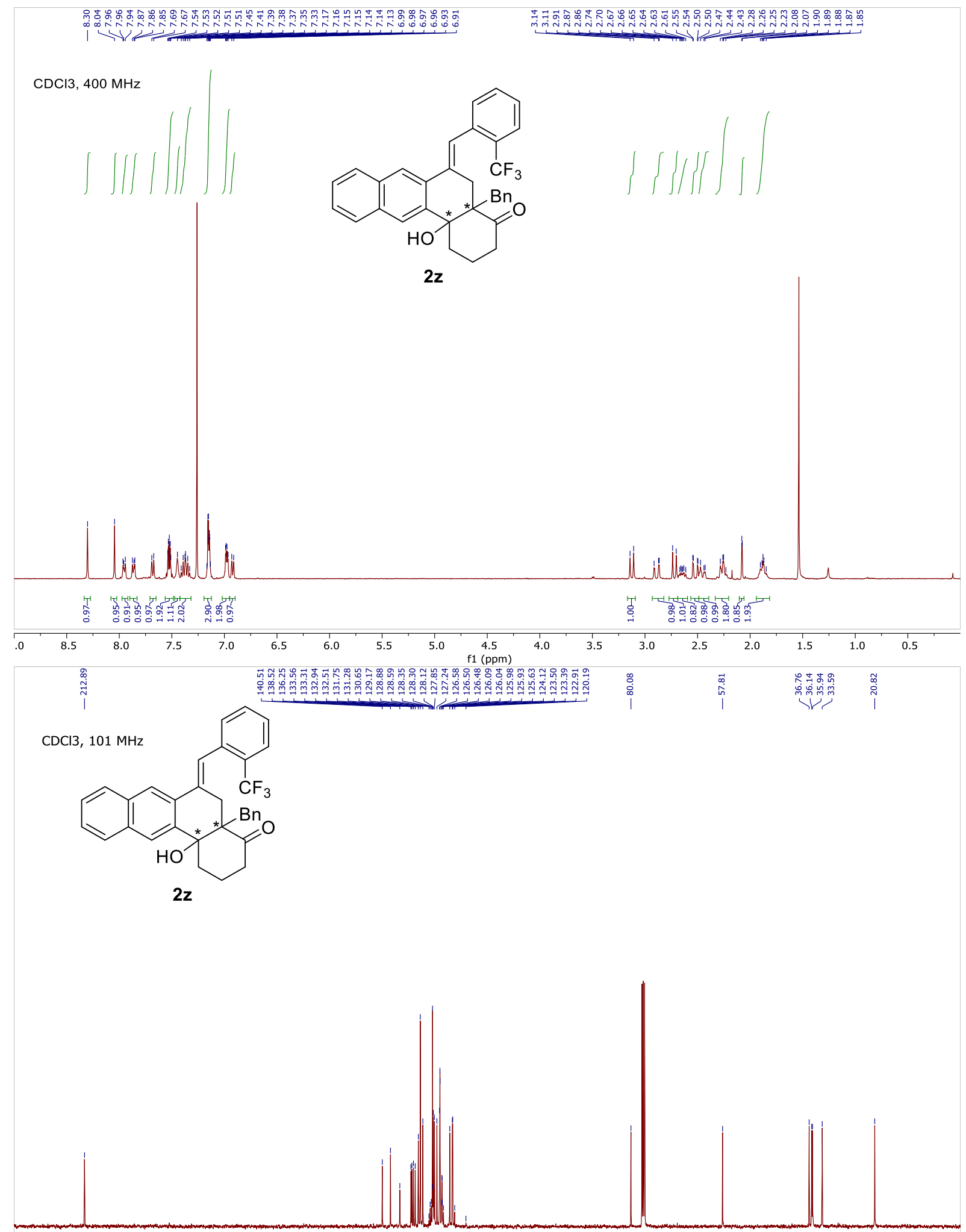

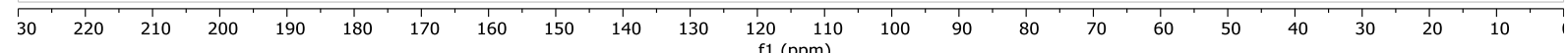


$\mathrm{CDCl} 3,400 \mathrm{MHz}$

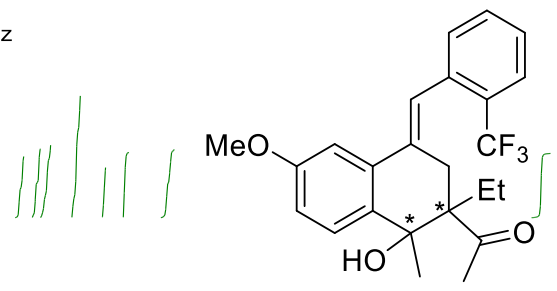

บับ

2aa
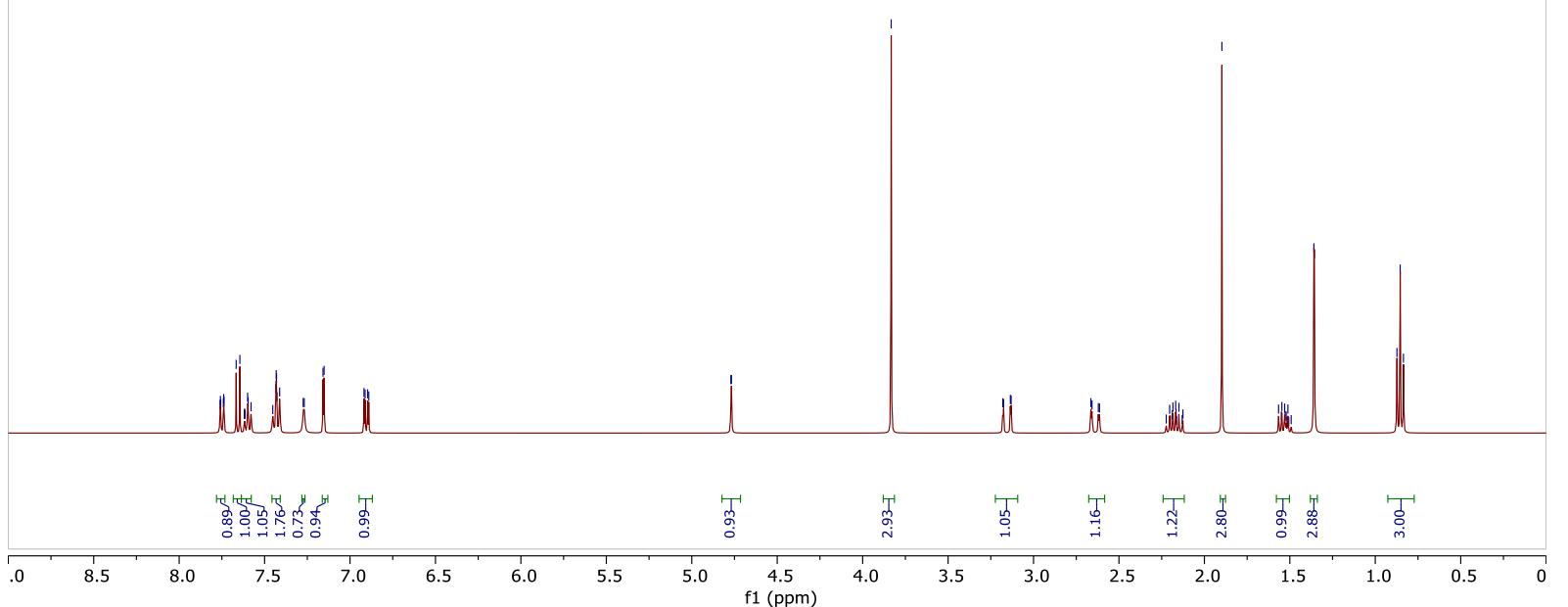

至

$\mathrm{CDCl} 3,101 \mathrm{MHz}$

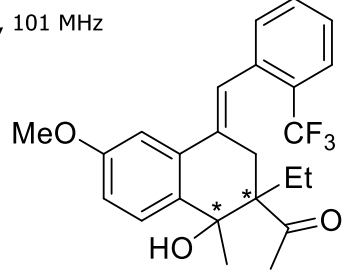

$2 \mathbf{a a}$

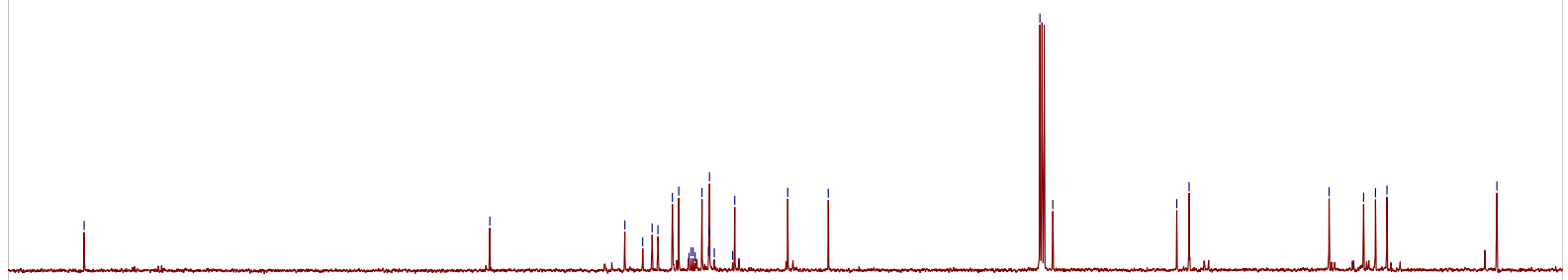

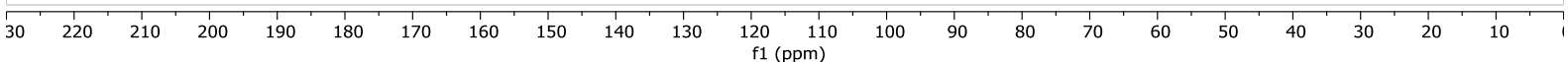




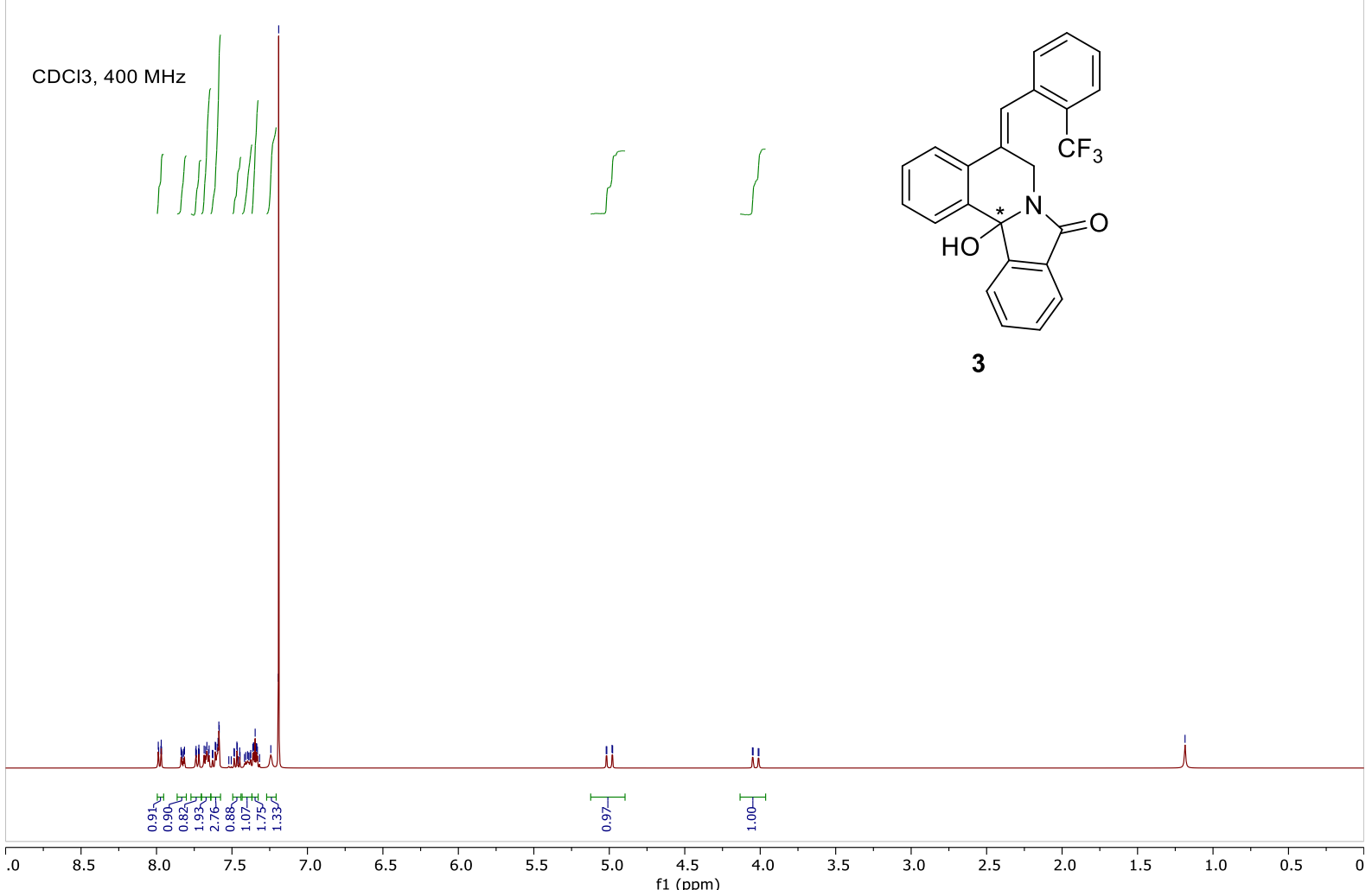

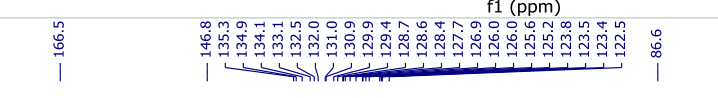

$\mathrm{CDCl} 3,101 \mathrm{MHz}$<smiles></smiles>

3

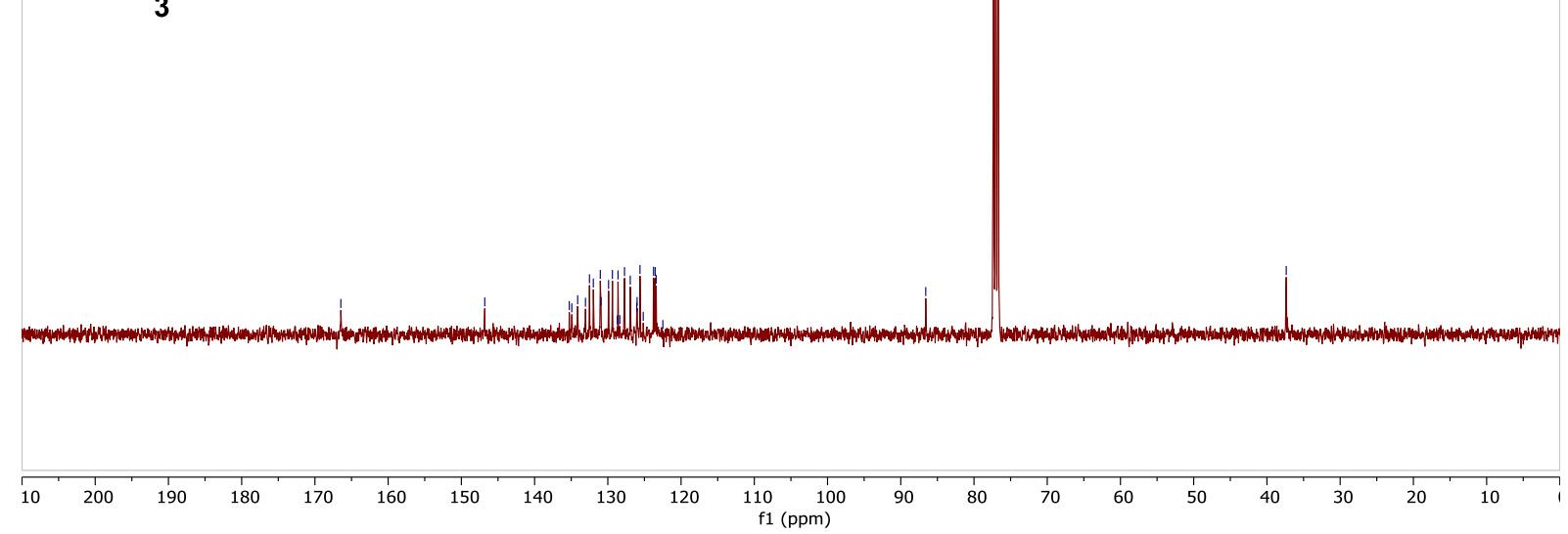




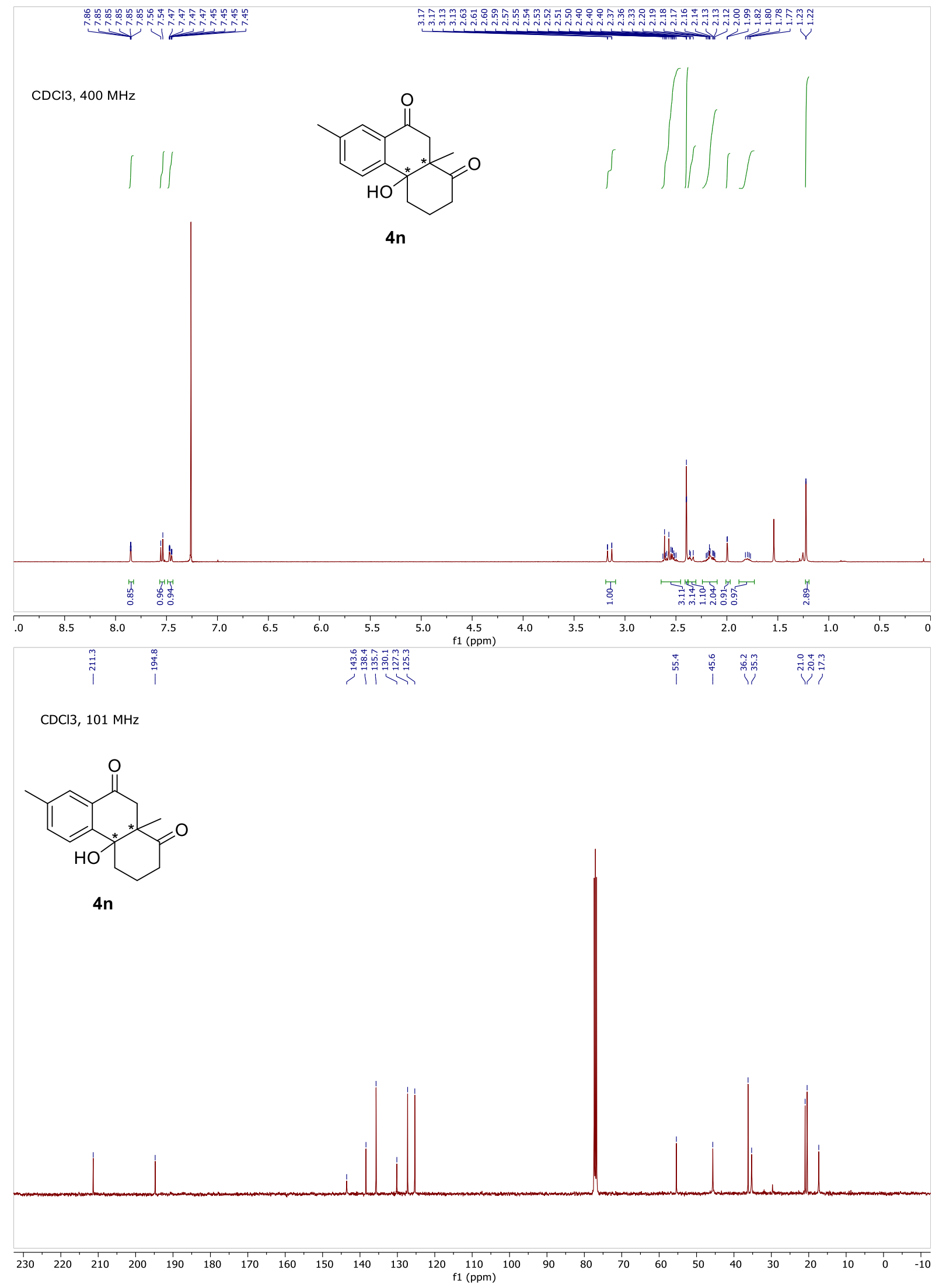



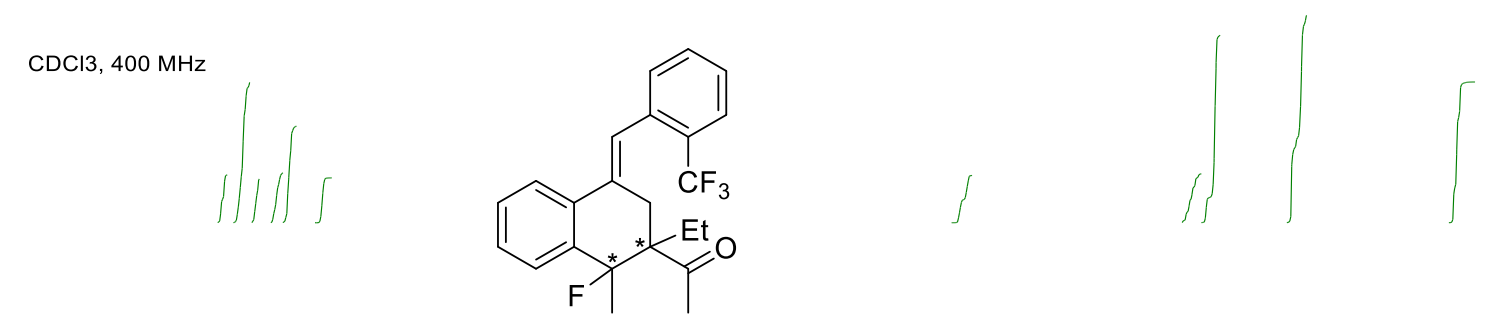

$5 e$

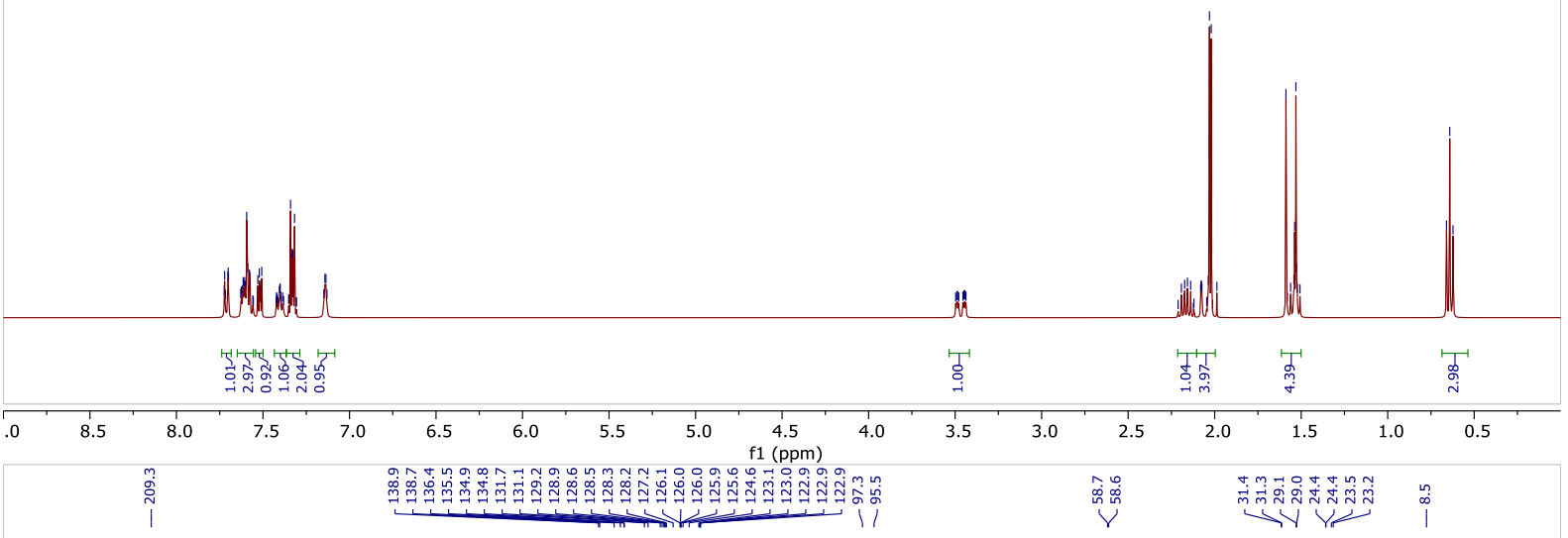

$\mathrm{CDCl} 3,101 \mathrm{MHz}$<smiles>CC(=O)C1(C(C)=O)CC(=Cc2ccccc2F)c2ccccc2C1(C)F</smiles>

$5 e$

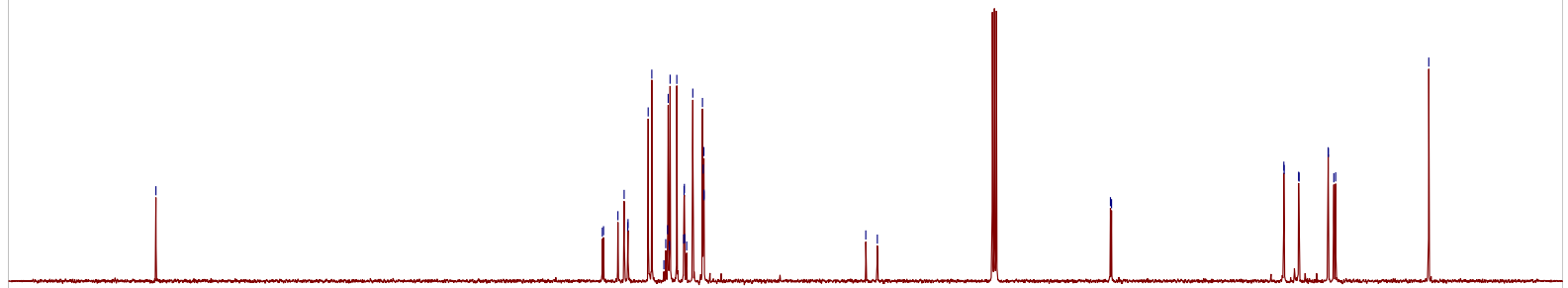

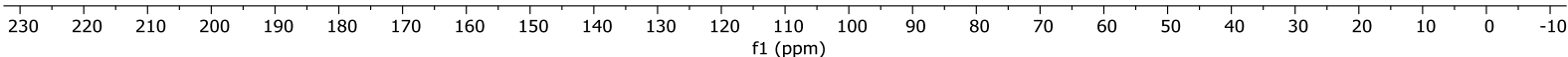

